\title{
National Ignition Campaign Program Completion Report
}
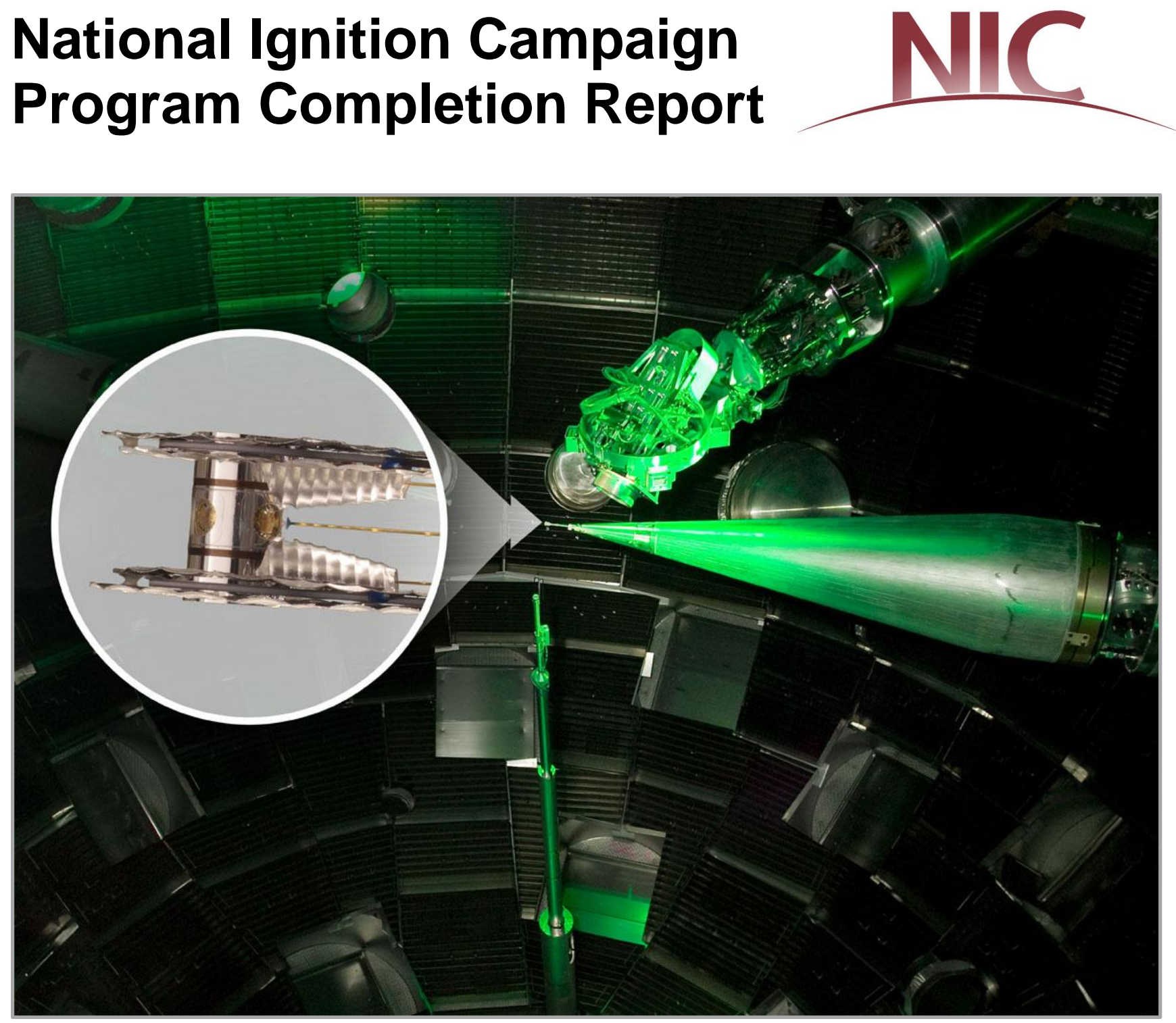

September 30, 2012

I1 Sational

LOS Alamos $\amalg$ Lawrence Livermore

\& GENERAL UR

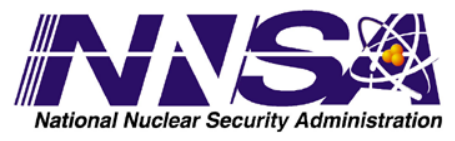




\section{National Ignition Campaign Program Completion Report}

\section{Disclaimer}

This document was prepared as an account of work sponsored by an agency of the United States government. Neither the United States government nor Lawrence Livermore National Security, LLC, nor any of their employees makes any warranty, expressed or implied, or assumes any legal liability or responsibility for the accuracy, completeness, or usefulness of any information, apparatus, product, or process disclosed, or represents that its use would not infringe privately owned rights. Reference herein to any specific commercial product, process, or service by trade name, trademark, manufacturer, or otherwise does not necessarily constitute or imply its endorsement, recommendation, or favoring by the United States government or Lawrence Livermore National Security, LLC. The views and opinions of authors expressed herein do not necessarily state or reflect those of the United States government or Lawrence Livermore National Security, LLC, and shall not be used for advertising or product endorsement purposes.

\section{Auspices Statements}

Work at General Atomics supported by the U.S. Department of Energy under Contract No. DEAC52-06NA27279.

Lawrence Livermore National Laboratory is operated by Lawrence Livermore National Security, LLC, for the U.S. Department of Energy, National Nuclear Security Administration under Contract No. DE-AC52-07NA27344.

Los Alamos National Laboratory is operated by Los Alamos National Security, LLC for the U.S. Department of Energy's National Nuclear Security Administration under Contract No. DE-AC5206NA25396.

Sandia National Laboratories is a multiprogram laboratory operated by the Sandia Corporation, a Lockheed Martin Company, for the U.S. Department of Energy under Contract No. DE-AC0494AL85000.

This work was supported by the U.S. Department of Energy Office of Inertial Confinement Fusion and the National Ignition Facility Project under Cooperative Agreement No. DE-FC5208NA28302 and the University of Rochester. The support of DOE does not constitute an endorsement of the views expressed in this document. 


\section{CONTENTS}

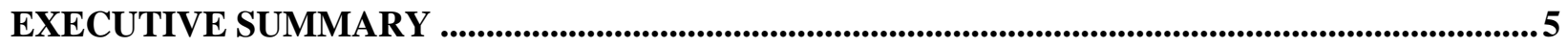

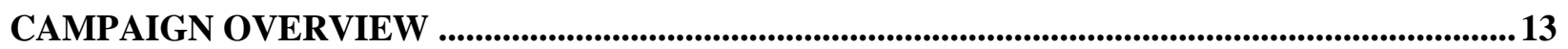

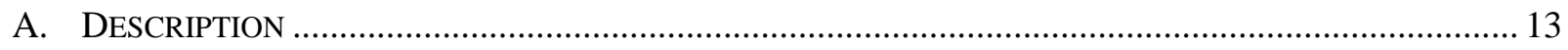

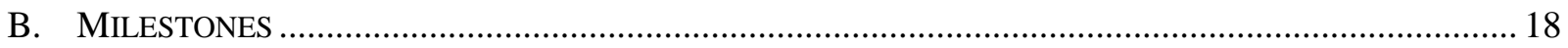

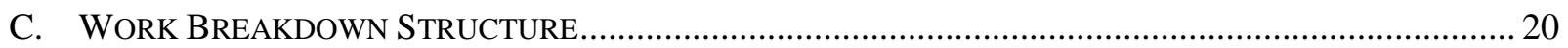

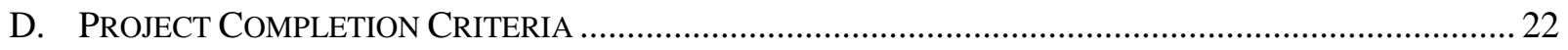

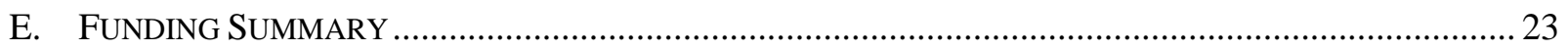

F. PARTICIPANT SCOPE SUMMARIES AND KEY MANAGEMENT PERSONNEL .................................... 24

SUMMARY OF KEY NIC ACCOMPLISHMENTS BY WBS.................................................26

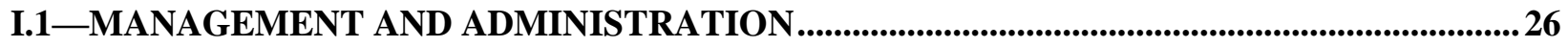

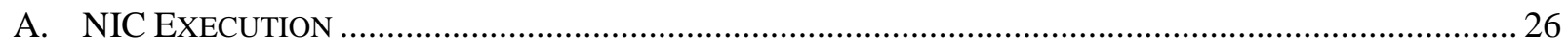

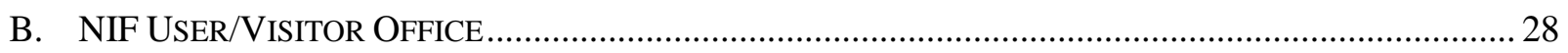

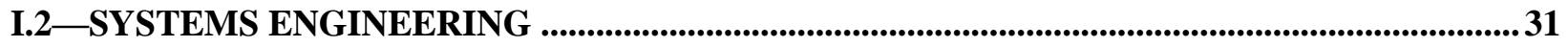

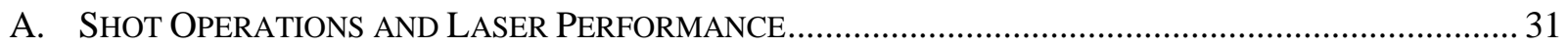

B. NIF INFORMATION TECHNOLOGY SOFTWARE TOOLS AND INFRASTRUCTURE .............................. 37

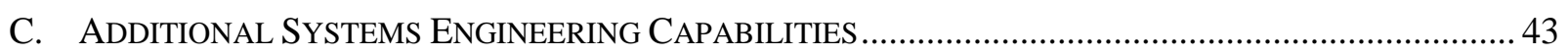

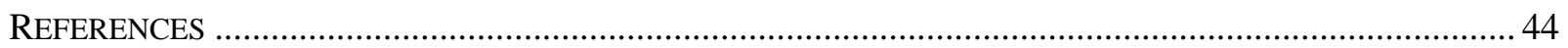

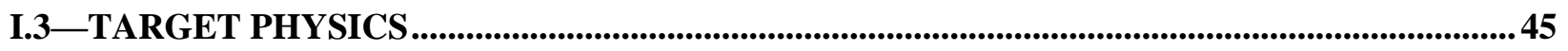

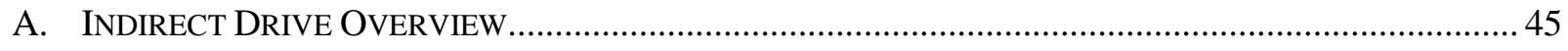

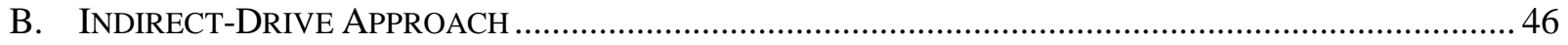

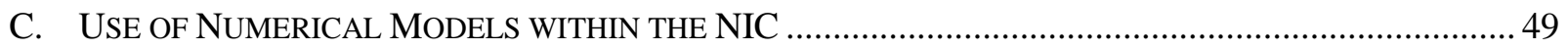

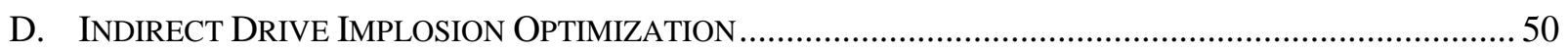

E. UsE OF ITFX AND FUEL STAGNATION PRESSURE TO ASSESS PROGRESS TOWARD ACHIEVING THE REQUISITE FUEL ASSEMBLY .......................................................................... 59

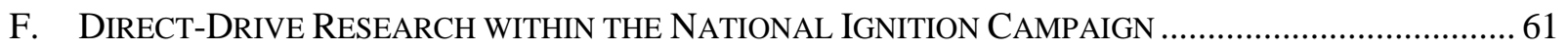

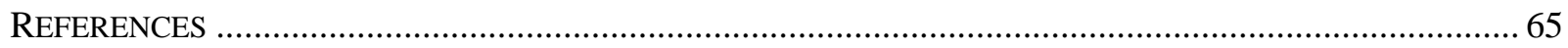

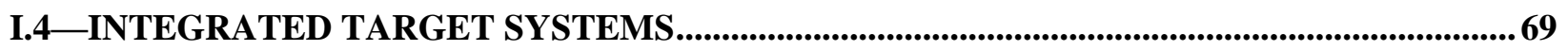

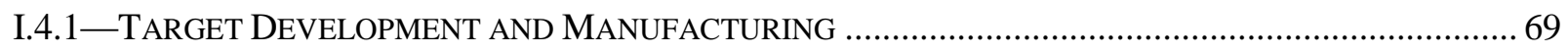

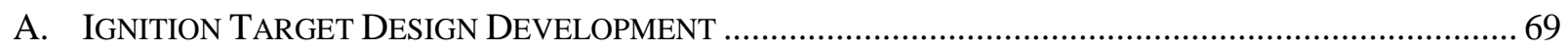

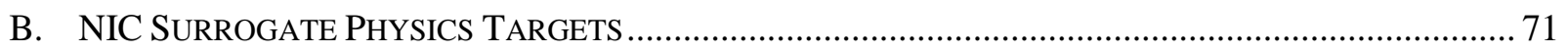

C. MAJOR TARGET MODIFICATIONS AND NEW TARGET DESIGNS …............................................... 72

D. CONTINUOUS IMPROVEMENTS IN TARGET QUALITY AND TARGET ASSEMBLY …........................... 73

E. TARGET FABRICATION AND MANUFACTURING INFRASTRUCTURE .............................................. 76

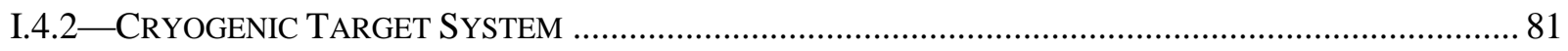

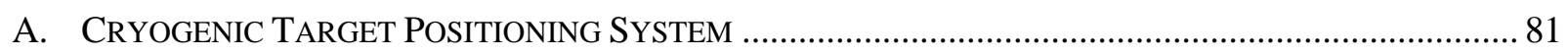

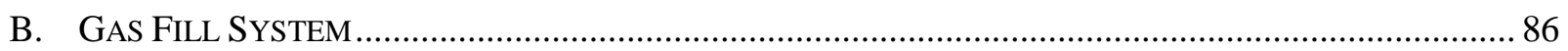

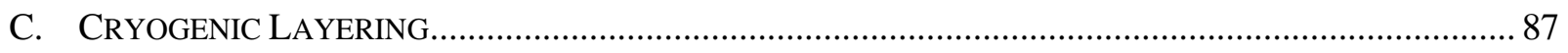

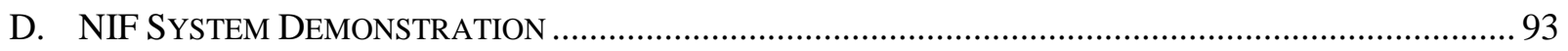

I.5-TARGET DIAGNOSTICS AND EXPERIMENTAL SYSTEMS ...............................................96

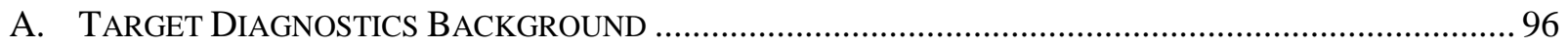




\section{National Ignition Campaign Program Completion Report}

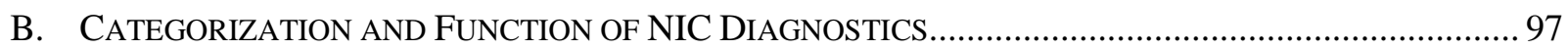

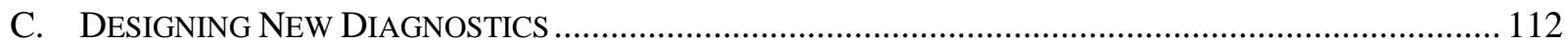

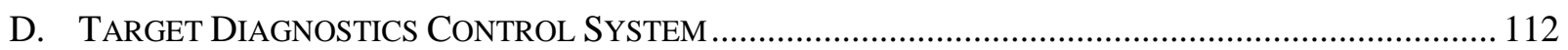

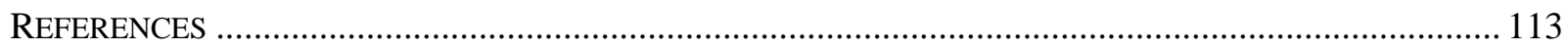

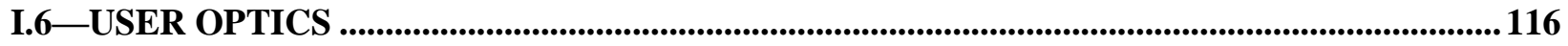

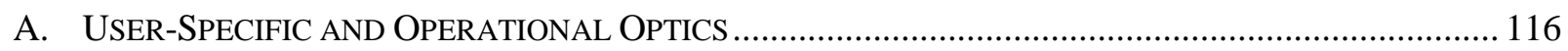

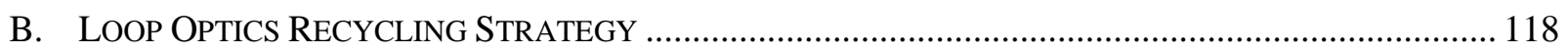

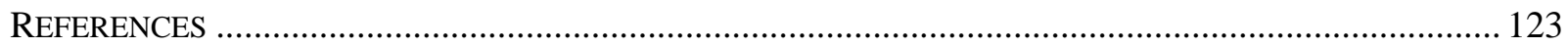

I.7-PERSONNEL AND ENVIRONMENTAL PROTECTION SYSTEMS ................................... 125

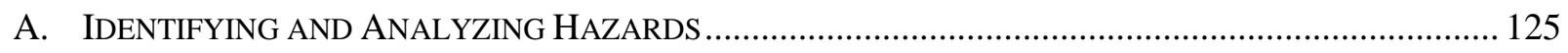

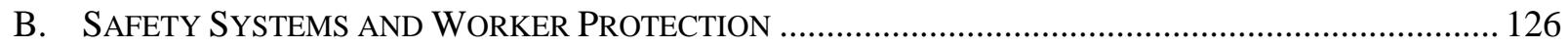

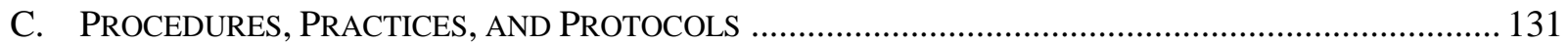

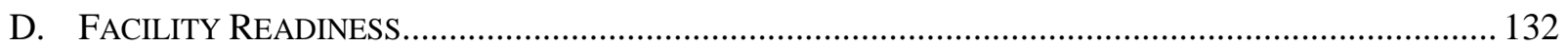

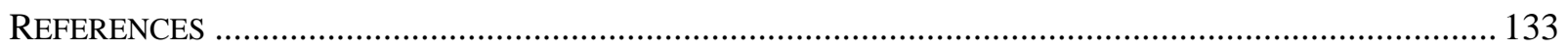

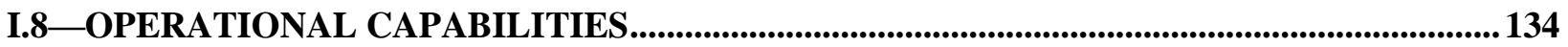

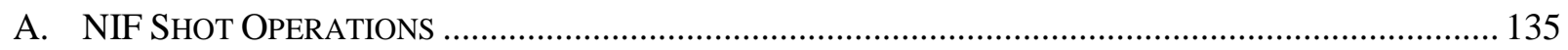

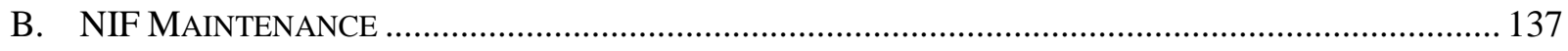

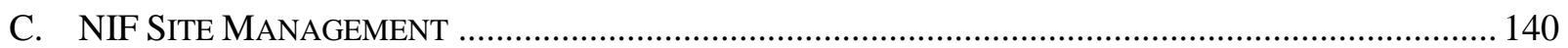

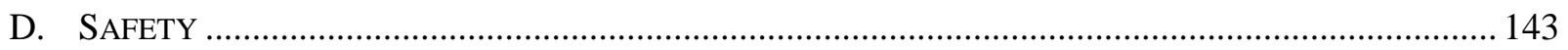

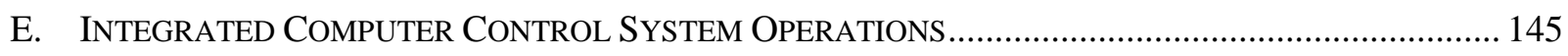

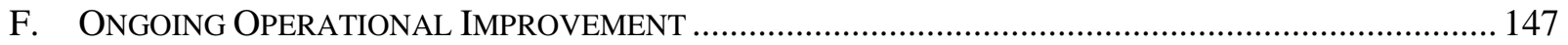

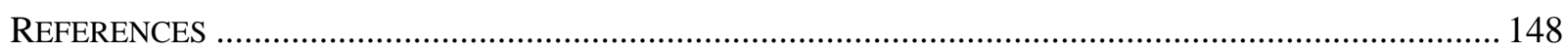

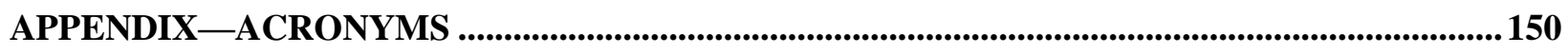




\section{National Ignition Campaign Program Completion Report}

\section{EXECUTIVE SUMMARY}

The National Ignition Campaign (NIC) was an integrated national effort and a partnership among the following: Livermore National Laboratory (LLNL), Los Alamos National Laboratory (LANL), Sandia National Laboratories (SNL), University of Rochester's Laboratory for Laser Energetics (LLE), and General Atomics (GA). Other key contributors include Massachusetts Institute of Technology (MIT), Lawrence Berkeley National Laboratory (LBNL), National Security Technologies (NSTec), Atomic Weapons Establishment (AWE) in England, and Commissariat a l'Energie Atomique (CEA) in France.

The objectives of the NIC Program were to achieve ignition and thermonuclear burn in the laboratory and to develop an ignition platform for ignition and HED applications on NIF by the end of FY2012. ${ }^{\mathrm{a}}$ It was also the intent of the NIC to transition the NIF to routine facility operations by the end of this period. The NIC was managed as an "enhanced management" activity within the National Nuclear Security Administration (NNSA). The NNSA requires enhanced management activities to adhere to a multi-year (beginning-to-end) cost and schedule baseline and a Work Breakdown Structure that is under formal change control and is documented in a formal execution plan. The efforts defined in the NIC Execution Plan Rev. 4.0 as necessary to meet the NIC objectives included developing, demonstrating, or executing:

- Developing an integrated ignition point design, with fusion energy output greater than or equal to laser energy delivered to the target

- Providing the required quantity of targets consistent with the ignition point design

- Providing a cryogenic target system capable of supporting the ignition point design at a shot rate consistent with the NIC shot plan

- Operating the NIF facility consistent with the requirements of the NIC experimental plan as the highest priority

- Providing laser beam characteristics consistent with target illumination specifications in the ignition point design

- Providing diagnostic systems to characterize laser/target illumination, hohlraum energetics, symmetry, ablator performance, shock timing, and fusion ignition

- Providing personnel and environmental protection systems and storage capabilities consistent with the NIC experimental plan, the Final Site-Wide Environmental Impact Statement for Continued Operation of Lawrence Livermore National Laboratory and Supplemental Stockpile Stewardship and Management Programmatic Environmental Impact Statement (SW/SPEIS), and the NIF Complex Tier 2 Safety Basis Document

- Planning and executing an integrated national effort, including experimental work at other facilities leading to experimental campaigns on NIF

- Conducting a direct-drive program to support the indirect-drive ignition effort and plan for a direct-drive program on NIF in the post-NIC period. This will include polar drive experiments in the current NIF configuration in the NIC program period.

- Providing short-pulse lasers for radiography and ignition applications

\footnotetext{
${ }^{a}$ A Baseline Change Proposal [BCP12-001] was approved by NNSA in FY2012 moving the 5 MJ milestone and thus effectively, the development of the ignition platform outside the scope of the NIC.
} 


\section{National Ignition Campaign Program Completion Report}

- Facilitizing, preparing for, and implementing routine NIF operations in support of non-ignition experiments for SSP, fundamental science and other missions

NIC formally ended on September 30, 2012. During NIC, 84 of its 86 Level 1 and 2 milestones were completed, leaving only two milestones incomplete: demonstration of limited alpha heating and ignition (Gain=1). Although fusion ignition and burn via inertial confinement fusion (ICF) on NIF-a goal that has long been recognized as a physics and technology grand challenge - was not achieved prior to the end of NIC, all other objectives, requirements, and program completion criteria described in its execution plan were accomplished, including:

- Demonstrating the NIF Functional Requirements and Primary Criteria ${ }^{\mathrm{b}}$ (FR\&PCs) for laser performance.

- Demonstrating NIC Program Completion Criteria (PCC) and the NIC PC\&FRs ${ }^{c}$ that provided the capabilities (diagnostics and targets) and facility infrastructure (cryogenic target system, optics, operational capabilities) for conducting ignition experiments.

- Implementing NIF governance as a national User Facility in support of ignition, HED Stewardship Science (HEDSS), and fundamental science, as well as other national security and inertial fusion energy applications.

- Transitioning NIF to routine facility operations, thereby establishing a unique laboratory capability for studying thermonuclear ignition and burn in dense deuterium-tritium (DT) plasmas and unique access to unprecedented high-pressure and high-density regimes.

The grand challenge of demonstrating fusion ignition and burn via ICF on NIF is ongoing. Great scientific progress during the campaign has advanced the study and science of ICF. In addition, the many technologies, capabilities, and processes developed and implemented during NIC have made NIF a unique world-class research capability, a mature facility that is well-equipped to continue the pursuit of ignition and its other missions. Some of these accomplishments are highlighted in the following sections.

Target physics. At the center of the NIC is the target point design. The target point design defines all of the elements needed to support the NIC experimental program (see Figure 1), including the laser, target, diagnostic, and facility requirements. The NIC target point design was developed using the world's most advanced high-performance computational systems, combining algorithms, models, and large-scale simulations with an extensive database of target physics information that has been gathered over the course of this campaign and previous programs.

b The scientific and engineering requirements for NIF were established and documented in the NIF FR\&PCs. In this document, NIF-0001006-OE (September 2006), the mission-related requirements and goals, as defined in the Justification of Mission Need, were translated into specific laser requirements such as laser energy, power, and a variety of beam characteristics. Capabilities for conducting experiments supporting the requirements of users with diverse needs and top-level operability, safety, and environmental requirements were also defined to ensure that, when completed, NIF would be operated in a manner consistent with its role as a national resource.

c Contained within Appendices D and F in the NIC EP Rev. 4 is the NIC PCC and the PC\&FRs, respectively. The NIC PCC and PC\&FRs represent the top-level system requirements that must be achieved to support the NIC objectives and to ensure that operations meet applicable federal, state, and local requirements for the protection of workers, the public, and the environment. 


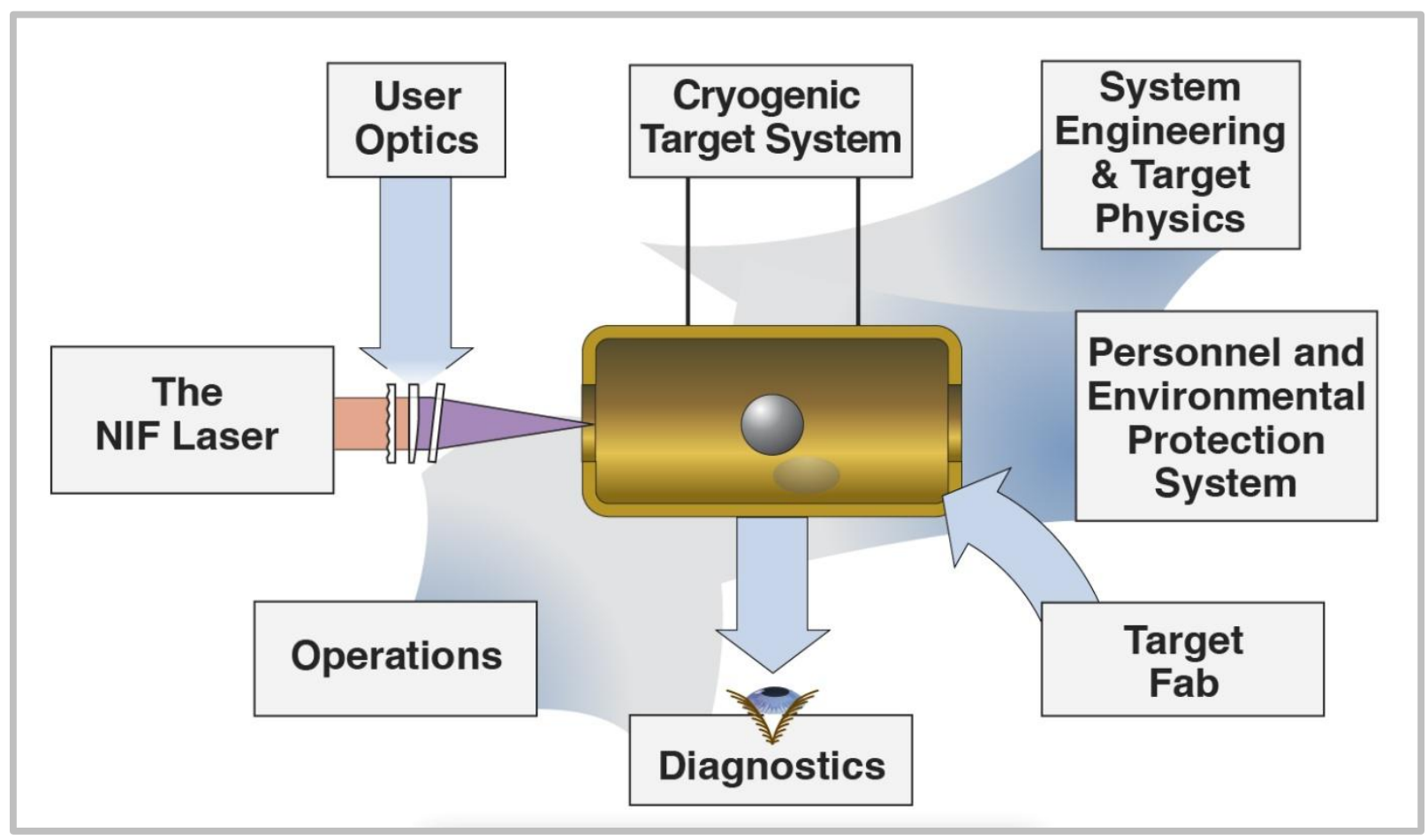

Figure 1. The elements of the National Ignition Campaign (NIC).

In July 2009, shortly after NIF project completion and demonstration of 1 MJ facility operations, NIC began executing a series of experiments. The experimental plan was carefully staged to systematically reduce physics uncertainties in the computational models used to predict the conditions needed for ignition while integrating diagnostics, targets, operations, and experimental capabilities in a phased manner. The first experiment using a cryogenic target (a gas-filled symmetry capsule) was conducted in September 2009, and the first implosion containing cryogenic layered fuel (a mix of tritium, hydrogen, and deuterium) occurred in September 2010, demonstrating for the first time the integration of the complex systems required for an ignition campaign. Precision capsule optimization experiments began in May 2011, after qualifying surrogate physics platforms to optimize specific performance parameters and working through technical difficulties encountered with laser entrance hole condensation and fuel layering. By the end of NIC, sixteen months later, significant progress had been made in controlling key implosion parameters - shape, velocity, adiabat, and mix - in cryogenic layered experiments.

Some of the achievements by the NIC team include:

- Hohlraum temperatures that exceed the $300 \mathrm{eV}$ point design goal with nearly constant laser energy coupling of $84 \pm 2 \%$ for energies from 1.2-1.8 MJ.

- Hot spot symmetry that meets ignition specifications using a combination of power balance and wavelength shifts between the inner and outer beams, and an additional wavelength shift between the two cones of inner beams.

- Accurate measurement of the dependence of implosion velocity on ablated mass, which is consistent with code simulations within the error bars. These measurements have enabled the identification of a mix performance boundary that depends on the velocity and the remaining unablated mass - a focus of the go-forward experimental plan.

- An increase in the fuel areal density ( $\rho r$ ) from $\sim 35 \%$ to $\sim 85 \%$ of that specified for the point design by implementing systematic improvements to the shock timing, hot spot symmetry, and laser pulse shape.

- A hot spot stagnation pressure is $\sim 40 \%$ of point design goals. 


\section{National Ignition Campaign Program Completion Report}

- An increase in the ignition threshold factor in recent experiments from less that 0.001 to just over 0.1 , over a factor of 100 . The ignition threshold factor is now within a factor of 3 of what is needed for significant alpha heating (where significant alpha heating has generally meant that the thermonuclear yield has doubled as a result of alpha deposition). The improved performance was achieved at a lower implosion velocity and laser power than the previous best-performing capsule, providing a greater margin for the path to ignition.

- For the first time in the laboratory, the beginning of alpha heating has been inferred. In the best implosions thus far, about $\sim 0.5 \mathrm{~kJ}$ of alpha particle energy is produced in the hot spot, the areal density of which is sufficient to trap a significant fraction of this energy. In these implosions, the total nuclear yield was $\sim 2.5 \mathrm{~kJ}$ which is also comparable to the thermal energy in the hot spot.

The NIC also included a direct-drive physics effort in support of the indirect-drive ignition program, which was led by LLE. Progress was made in understanding and improving the performance of symmetric direct-drive cryogenic target implosions during the NIC. Yields have increased for direct-drive implosions to over $10^{13}$ neutrons and ion temperatures up to $3 \mathrm{keV}$ were observed. Some of the physics models that are used for direct-drive ignition target designs were validated during NIC by a systematic study of target performance as a function of adiabat, in-flight aspect ratio, and implosion velocity.

Systems engineering. Systems Engineering was responsible for delivering the steady advances in laser performance, such as longer pulses and increased laser energy and power, required to execute the evolving NIC experimental plan. In the last year of the campaign, the NIF laser routinely delivered complex pulse shapes with between 1.45-1.8 MJ of energy and over $400 \mathrm{TW}$ of power. In July 2012, NIF demonstrated full NIF design requirements by executing an ignition pulse on target exceeding $1.8 \mathrm{MJ}$ and $500 \mathrm{TW}$. The facility is now capable of conducting over 50 high-energy, high-power shots annually in support of mission needs. But as impressive as the energy and power achievements have been, the crucial feature of NIF's laser performance is its precision.

NIF has delivered the requested $3 \omega$ energy and power for experiments within a few percent of request over a wide dynamic range of operating conditions. In large part, this has been due to the Laser Performance Operations Model (LPOM), a computational model that takes into account the energetic performance of each individual NIF beamline (differences in amplifier gains and optical transmission losses) to accurately determine the input conditions needed to generate the required output. LPOM, with exquisite precision and repeatability, satisfies user requests for pulse shape, energy, power, and power balance. LPOM is but one (albeit crucial) of the wide array of software and modeling tools developed during NIC. Through a host of servers, networks, databases, and storage devices, NIC has provided tools to support all phases of executing a shot on NIF-planning and scheduling an experiment, setup, execution, data archiving, visualization, and analysis. Many of these tools are available to NIF users.

Target development and manufacturing. The NIC experimental plan required two classes of targets: 1) targets used to optimize specific performance parameters (velocity, adiabat, shape, and mix), called surrogate physics targets, and 2) ignition targets used to assess integrated performance. The basic set of surrogate physics targets developed for NIC included Symmetry Capsules (to optimize symmetry or shape), Re-emit (to optimize early time symmetry), Keyhole (to optimize shock timing to set the adiabat), and Convergent Ablator (to optimize the tradeoff between velocity and mix). All of these targets were complex, consisting of many components that had to be made to stringent specifics with tight tolerances and precise alignment requirements. The ignition target was similarly intricate with the further requirement to grow and characterize a high-quality layer of frozen fuel inside.

The challenge for target fabrication was demonstrating that all of these targets could be fabricated with the requisite precision and quality and in the volume needed to keep pace with the experimental program. During the NIC, capabilities were established to manufacture capsules, hohlraums, and all the diagnostic and alignment components for NIC targets. Precision was maintained with new capabilities provided throughout the fabrication process, from the fuel fill tube - a tenth of the size of a human hair - to the 


\section{National Ignition Campaign Program Completion Report}

target error margin for micro-assembly of less than three microns. Throughout NIC, the agility of the target fabrication effort was stressed, as technicians, scientists, and engineers were asked to quickly respond to design and tolerance changes, such as capsule and ice surface smoothness. A small, but very important focused technology development program, with particular emphasis on precision engineering, characterization, and applied materials research, enabled the NIC target fabrication team to meet these challenges.

During the NIC, 210 targets were used in experiments, with all but 21 targets fielded at cryogenic temperatures. Of the total, 37 experiments were conducted using ignition targets with cryogenically layer fuel, by far the most difficult target to fabricate, assemble, and field. Target development and manufacturing groups at GA and LLNL worked seamlessly together during NIC, and their accomplishments are a significant achievement.

Cryogenic target system. During the NIC, the hardware and processes were put into place to field cryogenic layered fuel targets at target chamber center (TCC). The hardware, called CryoTARPOS (Cryogenic Target Positioner) system, is a complex, multifunctional system designed to cryogenically grow and characterize a high-quality cryogenic fuel layer inside an ignition target, position and align the target at TCC, and maintain layer quality until shot by the NIF laser. It initially took several weeks to grow and field an acceptable layer on CryoTARPOS for a NIC shot, and the process was heavily dependent on continuous support by engineers and scientists. Over the past two years, improvements in the hardware and characterization techniques, augmented by automation of the layering process, have evolved to the point where operations personnel can reliably produce a high-quality layer in four days, often with no participation of the scientific staff. The process for growing spherical, ultra-smooth hydrogen fuel layers free of small isolated defects has been refined through significant effort. Continuous improvement of the characterization process has resulted in near-real-time determination of layer quality with a high degree of confidence. The ability to make an early decision regarding the layer quality (whether to abort and restart the ice layering process) resulted in a considerable shot rate increase for layered implosions. Optimizing hydrogen crystal growth conditions to achieve the highest quality layers and developing a quick and effective layer quality evaluation method were among the most critical accomplishments during NIC.

Diagnostics and experimental systems. NIF is now equipped with approximately 60 optical, $\mathrm{x}$-ray, and nuclear gamma ray, activation, and neutron diagnostics that together provide 300 channels for data acquisition to support the experimental campaigns and measure laser and target performance. Many instruments implemented on NIF have state-of-the-art adaptations of previous diagnostics developed for the nuclear test program or other laser facilities; others are entirely novel. An essential element of the overall plan for NIF diagnostics was ensuring that several diagnostics could measure the same characteristic (observable) to provide redundancy and ensure measurement accuracy. The comprehensive suite of NIF diagnostics was developed as part of a successful collaboration among eleven institutions that shared the responsibility for design, construction, and diagnostic accuracy. Given the diversity of experimental requirements and space constraints around the target chamber, many of the instruments were designed to be removable and exchangeable; the use of standardized Diagnostic Instrument Manipulators (DIMs) by several of the NIC partners has meant that the removable diagnostics could be easily tested at other facilities such as OMEGA before implementation at NIF. A steady increase in the number of diagnostics and their performance over the course of the NIC has supplemented the quantity and quality of data gathered from every experiment. The extraordinary contributions by the NIC partners (LANL, LLE, SNL, GA, and LLNL) and collaborators (MIT, LBNL, NSTec, AWE, and CEA) to NIF diagnostics are another success story from NIC.

User optics. Critical optical components were developed and manufactured during NIC to support experiments for the ignition program on NIF. These optics have consistently met rigorous specifications for flatness, uniformity, and their ability to withstand intense laser fluences. In particular, the NIF's $3 \omega$ fused silica final optics are the most susceptible to optical damage and have extremely challenging optical 


\section{National Ignition Campaign Program Completion Report}

specifications, making them difficult to fabricate and relatively expensive. The $3 \omega$ optics recycling loop, a strategy for economically maintaining and reusing these optics, has been very effective in supporting and enabling routine high-energy, high-power experiments on NIF. Implementation of the loop strategy was guided by an LLNL supported research program that explored understanding basic phenomenology of laser-induced optical damage. This research led to significantly better methods for arresting damage initiation, developing higher quality and more robust bulk materials for optics fabrication, as well as advanced finishing techniques and post-fabrication processes that further improved damage resistance. This work guided the selection of technologies and processes implemented as part of the Loop strategy under NIC and enabled NIF $3 \omega$ optics to operate consistently and predictably at a level significantly above their damage growth threshold.

Improvements in the critical technologies needed for operating the loop, the improved surface finishing, in-situ optics inspection, and damage growth mitigation have enabled a steady increase in NIF laser performance from about $300 \mathrm{~kJ}$ in July 2009 to almost 2 MJ in July 2012. Before this work, optical damage was a seemingly intractable problem for those designing and operating high-energy, high-power laser systems. The advancements made in understanding on a fundamental level the causes and signatures of optical damage and the development of new technologies to arrest and mitigate damage, coupled with the innovative loop recycling strategy, have positioned NIF to operate routinely in regimes far beyond comparable laser systems.

Personnel and environmental protection systems. Since the use of radioactive and/or hazardous materials such as tritium (part of the fusion fuel used in ignition targets) during routine NIF operations is a requirement of NIC, the necessary safety systems to monitor, contain, and process these materials (e.g. tritium processing system and the hazardous waste management area) were put in place. NIF and its personnel protective systems (shield doors, radiation monitoring equipment, and interlock systems), in conjunction with training and work authorization processes, have proved highly effective at protecting workers, the public, and the environment, as well as sensitive equipment and instruments. NIF has over 600 qualified radiological workers and teams of radiological safety specialists to ensure that radiation hazards at NIF are controlled and maintained at ALARA ${ }^{\mathrm{d}}$ levels.

Operational capabilities. During the NIC, the operations, planning, training, and maintenance aspects of the NIF were established. This component included every aspect associated with performing an experiment: setting up the laser and diagnostics, aligning the target, executing the experiment, and collecting and archiving the data. The objective was to provide high-quality stewardship capabilities over the 30-year expected operational lifetime of the facility. One of the key operational accomplishments during NIC was development of NIF's Integrated Computer Control System, the most complex, real-time control system ever designed for scientific research. The automated control system provides reliable monitoring and control of approximately 60,000 distributed control points; the precise orchestration of these control points results in a safe, precise, and well-diagnosed laser shot. Throughout the NIC, NIF operations staff worked to evaluate and improve operational processes and capabilities, with the intent of maximizing facility reliability, availability, and maintainability while preserving safety. For instance, a reliability-centered maintenance program was recently deployed to reduce equipment failure rates, improve equipment repair and response times, anticipate problems, and look for windows of opportunity to conduct routine maintenance. Using a reliability-centered process when making maintenance resource determinations allowed NIF personnel to identify and focus on critical functions that impact the shot cycle and shot rate. Safe and efficient operational processes and capabilities developed during the NIC have enabled NIF staff to maximize the data return on each shot performed.

d ALARA = As Low As Reasonably Achievable. 


\section{National Ignition Campaign Program Completion Report}

Management and administration. The planning, management, and oversight component of the NIC involved establishing and managing work scope, schedule, and costs and coordinating NIC-related activities with the participating partner sites. A significant part of the management and administration effort for the NIC program involved regular communication with stakeholders, federal program managers, and the NIC partner sites through reports, letters, websites, and other documentation. The NIC also actively participated in a number of technical reviews, and program progress was communicated to the scientific community through presentations at national and international conferences.

Under NIC, NIF transitioned from a project to routine operations as a user facility. As part of the transition, the NIF User/Visitor Office was established to help foster a fundamental science user community on NIF and establish the NIF User Group. The Office developed NIF governance and facility use policies, and put in place processes for proposal submission and review to ensure that the highest quality science experiments are conducted on NIF. The first proposal solicitation for fundamental science experiments at the NIF was issued in late 2009, and several successful proposals have been initiated. Establishing governance for NIF as a national user facility for HED science was a notable achievement of the NIF User/Visitor Office under NIC.

Conclusion. NIC developed the capabilities necessary to perform stockpile stewardship, fundamental science, and other national security missions and demonstrated these capabilities on the NIF in a series of 314 target experiments. Although ICF ignition and burn on NIF was not achieved prior to the end of NIC, all other objectives, requirements, and program completion criteria were accomplished, including:

- Demonstrating the NIF FR\&PCs ${ }^{\mathrm{e}}$ for laser performance.

- Demonstrating the NIC PC\&FRs ${ }^{\mathrm{f}}$, which encompassed the capabilities (diagnostics and targets) and facility infrastructure (cryogenic target system, optics, operational capabilities) for conducting ignition experiments.

- Implementing NIF governance ${ }^{\mathrm{g}}$ as a national User Facility in support of ignition, HEDSS, and fundamental science, as well as other national security and inertial fusion energy applications.

- Transitioning NIF to routine facility operations, thereby establishing a unique laboratory capability for studying thermonuclear ignition and burn in dense DT plasmas and unique access to high-pressure and high-density regimes.

Figure 2 highlights the elements of the NIC program delivered at the end of the campaign.

e National Ignition Facility Function Requirements \& Primary Criteria - Evidence of Completion, LLNL-AR592152, NIF-0135506

f National Ignition Campaign Program Completion Criteria and Primary Criteria \& Functional Requirements Evidence of Completion, LLNL-AR-592152, NIF-0135596

g National Ignition Facility Governance Plan, LLNL-AR-416565, NIF-0115829-AA 


\section{National Ignition Campaign Program Completion Report}

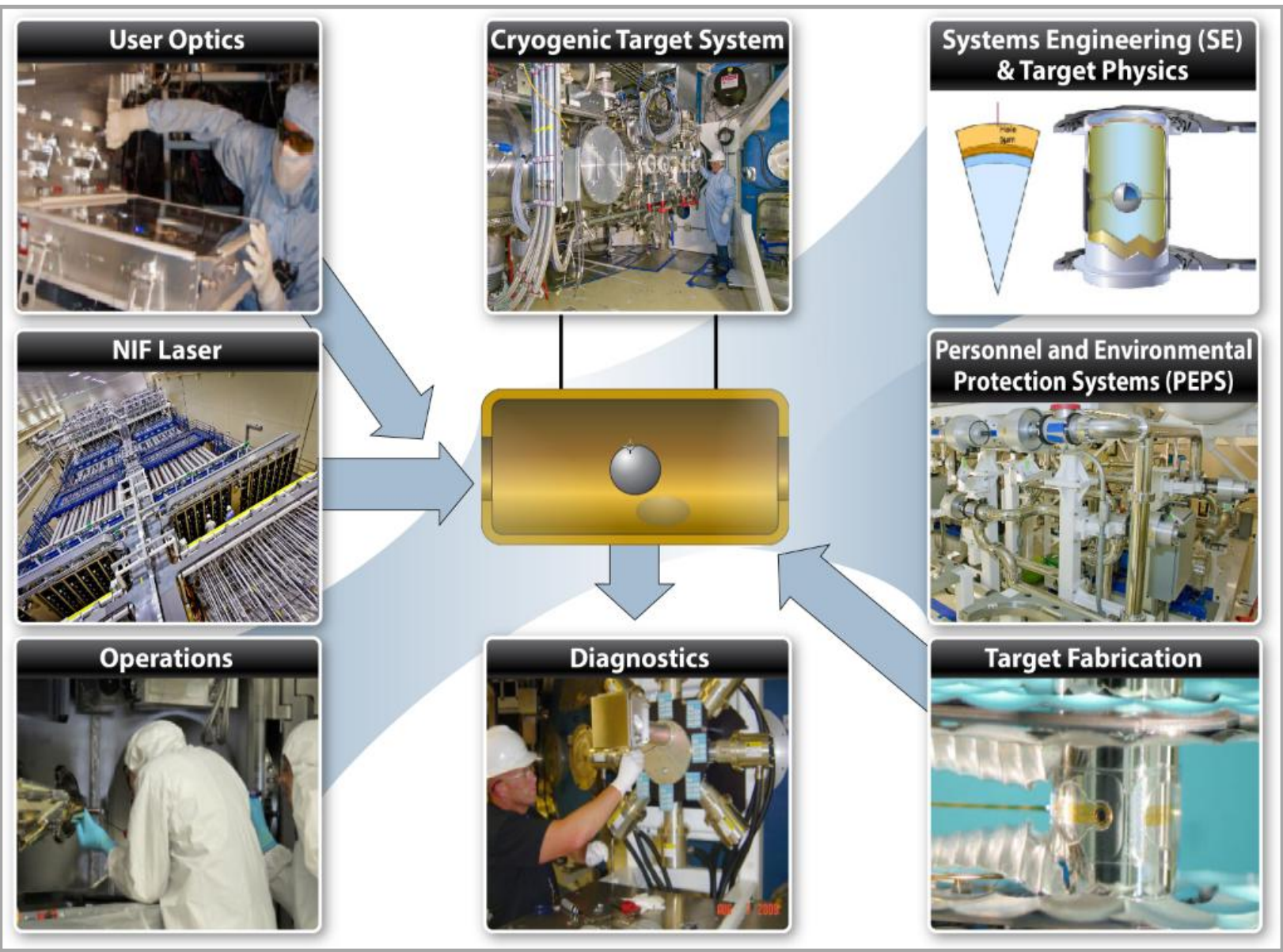

Figure 2. The elements of the NIC program delivered at the end of the campaign. 


\section{National Ignition Campaign Program Completion Report}

\section{CAMPAIGN OVERVIEW}

\section{A. Description}

The National Nuclear Security Administration (NNSA) established the Stockpile Stewardship Program (SSP) in the mid-1990s as a single, highly integrated technical program for maintaining the safety and reliability of the U.S. nuclear stockpile in an era without nuclear testing. The National Ignition Campaign (NIC) represented a significant component of the SSP. The objectives of the NIC Program were to achieve ignition and thermonuclear burn in the laboratory and to develop an ignition platform for ignition and HED applications on NIF by the end of FY2012. ${ }^{\mathrm{h}}$ It was also the intent of the NIC to transition the NIF to routine facility operations by the end of this period.

NIC was an integrated national effort among the following institutions: Lawrence Livermore National Laboratory (LLNL), Los Alamos National Laboratory (LANL), Sandia National Laboratories (SNL), University of Rochester's Laboratory for Laser Energetics (LLE), and General Atomics (GA). Other key contributors included Massachusetts Institute of Technology (MIT), Lawrence Berkeley National Laboratory (LBNL), National Security Technologies (NSTec), Atomic Weapons Establishment (AWE) in England, and Commissariat a l'Energie Atomique (CEA) in France. NIC was managed as an "enhanced management" [1] activity within the NNSA. The NNSA requires enhanced management activities to adhere to a multi-year (beginning-to-end) cost and schedule baseline and a Work Breakdown Structure (WBS) that is under formal change control and is documented in a formal execution plan (EP).

During the spring and early summer of 2005, as the EP was being prepared, an extensive review of the NIC experimental plan [2] was conducted by the JASON at the request of NNSA. In late June 2005, the NIC EP (Rev. 0) [3] was signed by all participating sites (LLNL, LANL, SNL, LLE, and GA) and NNSA, and shortly thereafter, was submitted to Congress. Earned value reporting for NIC began in FY2006. The final plan was NIC EP Rev. 4.0, which was signed by NNSA in January 2010.

The efforts defined in the NIC EP as necessary to meet the NIC objectives included:

- Developing an integrated ignition point design, with fusion energy output greater than or equal to laser energy delivered to the target

- Providing the required quantity of targets consistent with the ignition point design

- Providing a cryogenic target system capable of supporting the ignition point design at a shot rate consistent with the NIC shot plan

- Operating the NIF facility consistent with the requirements of the NIC experimental plan as the highest priority

- Providing laser beam characteristics consistent with target illumination specifications in the ignition point design

- Providing diagnostic systems to characterize laser/target illumination, hohlraum energetics, symmetry, ablator performance, shock timing, and fusion ignition

- Providing personnel and environmental protection systems and storage capabilities consistent with the NIC experimental plan, the Final Site-Wide Environmental Impact Statement for Continued Operation of Lawrence Livermore National Laboratory and Supplemental Stockpile Stewardship and Management Programmatic Environmental Impact Statement (SW/SPEIS), and the NIF Complex Tier 2 Safety Basis Document

h A Baseline Change Proposal [BCP12-001] was approved by NNSA in FY2012 moving the 5 MJ milestone and thus effectively, the development of the ignition platform outside the scope of the NIC. 


\section{National Ignition Campaign Program Completion Report}

- Planning and executing an integrated national effort, including experimental work at other facilities leading to experimental campaigns on NIF

- Conducting a direct-drive program to support the indirect-drive ignition effort and plan for a direct-drive program on NIF in the post-NIC period. This will include polar drive experiments in the current NIF configuration in the NIC program period.

- Providing short-pulse lasers for radiography and ignition applications

- Facilitizing, preparing for, and implementing routine NIF operations in support of non-ignition experiments for SSP, fundamental science and other missions

NIC point design, experimental capabilities, and planning: In FY2006, NIC began addressing the objectives described above. Efforts at the beginning of NIC were concentrated on three fronts concurrently:

- Developing the integrated ignition target point design, with fusion energy output greater than or equal to laser energy delivered to the target. These efforts utilized the world's most advanced high-performance computational systems, combining algorithms, models, and large-scale simulations with a large database of target physics information gathered and validated over the course of three decades of laser experiments.

- Designing, fabricating, and procuring the diagnostic systems, cryogenic target system, optical components, and other facility infrastructure required to operate NIF at high fluences and conduct ignition experiments with yield. A major effort was launched to develop capabilities to fabricate capsules, hohlraums, and all the diagnostic and alignment components for ignition targets and targets required for supporting experiments. This encompassed the requisite equipment and technology development for manufacturing and assembling a wide variety of targets to stringent specifications and characterizing the target components and assemblies to exquisite precision. An institutionally supported research and development program to develop a fundamental understanding of optical damage helped to guide the selection of technologies and processes implemented as part of the Loop strategy under NIC. These efforts have enabled NIF $3 \omega$ optics to operate consistently and predictably at a level significantly above their damage growth threshold.

- Developing an experimental plan to be carried out on NIF with the ultimate goal of achieving ignition. The experimental plan was carefully staged to systematically reduce physics uncertainties in the computational models used to predict the conditions needed for ignition, while integrating diagnostics, targets, operations, and experimental capabilities in a phased manner.

NIF project completion: During this same period, the NIF project was installing the remaining linereplaceable units, developing and testing the integrated computer control system, and progressing toward project completion. On March 10, 2009, NIF delivered 1.1 MJ of energy at $3 \omega(351 \mathrm{~nm})$ to target chamber center (TCC) using all 192 beams, becoming the world's first megajoule laser facility. March 27, 2009 marked the signing of Critical Decision 4 and the start of NIF's transition from a project to an operational facility. Over the next several months, the initial set of diagnostics to measure laser absorption and hohlraum conditions (drive diagnostics) was installed as NIF methodically executed shots to calibrate, synchronize, validate beam pointing, and assess scattered light in the target chamber in preparation for target physics experiments.

Target physics experiments: Target physics experiments commenced in July 2009 with warm target shots and commissioning of newly installed diagnostics in preparation for the first cryogenic implosion of a gas-filled Symmetry Capsule (Symcap), which was conducted in September 2009. The hohlraum energetics campaign that followed resulted in the successful commissioning of a $300 \mathrm{eV}$ plastic $(\mathrm{CH})$ point design hohlraum and demonstrated that cross-beam energy transfer between NIF's inner and outer cones of beams could be used to change the symmetry of implosions. In addition, the diagnostics for characterizing laser-hohlraum coupling were commissioned. 


\section{National Ignition Campaign Program Completion Report}

Ignition Preparation Period: The hohlraum energetics campaign was completed in early December 2009, beginning an approximately seven-month-long Ignition Preparation Period (IPP). During the IPP, several hundred laser shots were executed that were essential for calibrating beam diagnostics and refining the parameters in the Laser Performance Operations Model (LPOM), improving pointing accuracy, validating focus at TCC, and qualifying new hardware. Major equipment installed and commissioned during the IPP included the cryogenic target positioner for fielding targets at NIF with layered fusion fuel, the Personnel and Environmental Protection Systems (PEPS) for providing proper handling of tritium and other potentially hazardous materials, and the target diagnostic system (the initial set of target response/implosion, hot spot, and areal density diagnostics to support the subsequent capsule optimization and ignition campaigns). A third oscillator also was added to the NIF during this period - a significant new capability. The availability of three wavelengths rather than the previous two provided more control over crossbeam energy transfer between the various cones ${ }^{i}$ of NIF beams and thus more control over capsule implosion symmetry.

First integrated implosion experiment: In late July, the IPP ended and commissioning for the new NIF hardware began; this included the cryogenic target positioner (CryoTARPOS) and an array of new diagnostic systems. On September 29, 2010, the first implosion of a capsule containing cryogenic layered fuel (a mix of tritium, hydrogen, and deuterium) was conducted, demonstrating for the first time the integration of the complex systems required for an ignition campaign. From October 2010 through midFebruary 2011, efforts focused on the qualification of the various surrogate physics platforms to be used for precision optimization of the NIC ignition point design target. The neutron imaging system was added, as were nuclear diagnostics that were needed for higher yield $\left(<10^{17}\right.$ neutrons). In this period, some technical difficulties were encountered - in particular, layered fuel production difficulties and frozen condensation formation on the laser entrance hole (LEH) windows that affected shock timing experiments. Introducing a second LEH window (called a storm or warm window), resolved the condensation problem. The layering problem was addressed by introducing hardware and protocol changes to improve the cryogenic fuel layering capability.

HED Stewardship Science (HEDSS) campaign: From mid-February through mid-March 2011, NIF was primarily dedicated to conducting shots supporting HEDSS; 35 shots were performed, representing significant progress toward successfully completing one Level 1 and three Level 2 milestones. These experiments were followed by a six-week NIF Facility Maintenance and Reconfiguration (FMR) period. During the FMR, specific laser performance and facility improvements (including radiation-hardened diagnostics) were implemented to prepare NIF for precision optimization and experiments with higher yield (up to $10^{19}$ neutrons).

Precision capsule optimization campaign: Precision implosion experiments began in May 2011 with the goal of optimizing the key parameters of implosion shape, velocity, adiabat, and mix. Campaigns focusing on increasing the shell velocity and pressure at stagnation, improving the implosion shape, and assessing mix continued for the next 16 months, with the exception of a 5-week FMR conducted in May 2012 to prepare NIF for 500 TW/1.8 MJ operations. During this FMR, the beam aperture was increased and amplitude modulation/frequency modulation compensators were added. These enhancements enabled NIF to demonstrate completion of its FR\&PCs, an important NIC deliverable. On July 5, 2012, NIF delivered $1.82 \mathrm{MJ}$ of energy and $523 \mathrm{TW}$ of power in a shaped ignition pulse to a target, exceeding its design requirements. In the period between the start of precision experiments and the end of NIC, significant progress was made in controlling key implosion parameters through the execution of 126 target physics experiments, of which 33 were cryogenic layered experiments.

i For 3-color operation, the two inner cones of beams (23.5 and the 30 degree beams) are operated at slightly different wavelengths, with the outer cones of beams (44 and 50 degree beams) operating at a third wavelength. 


\section{National Ignition Campaign Program Completion Report}

NIC completion: NIC formally ended on September 30, 2012. Of NIC's 86 Level 1 and Level 2 milestones, 84 were completed during NIC, leaving only two milestones incomplete: demonstration of limited alpha heating and ignition (Gain=1). Although fusion ignition and burn via ICF on NIF-a goal that has long been recognized as a physics and technology grand challenge-was not achieved prior to the end of NIC, all other objectives, requirements, and program completion criteria were accomplished, including:

- Demonstrating the NIF FR\&PCs ${ }^{\mathrm{j}}$ for laser performance.

- Demonstrating NIC Program Completion Criteria (PCC) and the NIC PC\&FRs ${ }^{\mathrm{k}}$ that provided the capabilities (diagnostics and targets), and facility infrastructure (cryogenic target system, optics, operational capabilities) to conduct ignition experiments.

- Implementing NIF governance [4] as a national User Facility in support of ignition, HEDSS, and fundamental science, as well as other national security and inertial fusion energy applications.

- Transitioning NIF to routine facility operations, thereby establishing a unique laboratory capability for studying thermonuclear ignition and burn in dense deuterium-tritium (DT) plasmas and unique access to unprecedented high-pressure and high-density regimes.

The summary of NIF system shots conducted during NIC from November 2008 through September 2012 is provided in Table 1.

Table 1. NIC system shot summary.

\begin{tabular}{|c|c|c|c|c|c|}
\hline Type & Specific Purpose & Cryo & Layer & Warm & Total \\
\hline $\begin{array}{l}\text { Target shots- } \\
\text { Program Data }\end{array}$ & NIC & 152 & 37 & 21 & 210 \\
\hline \multirow[t]{3}{*}{$(314=30 \%)$} & HEDSS & & & 80 & 80 \\
\hline & Nat'I Security Applications & & & 13 & 13 \\
\hline & Fundamental Science & & & 11 & 11 \\
\hline $\begin{array}{l}\text { Target shots- } \\
\text { Capabilities }\end{array}$ & $\begin{array}{l}\text { Target Diagnostics } \\
\text { Commissioning/Calibration }\end{array}$ & & & 110 & 110 \\
\hline$(152=15 \%)$ & $\begin{array}{l}\text { Laser Commissioning/ } \\
\text { Calibration }\end{array}$ & & & 42 & 42 \\
\hline $\begin{array}{l}\text { Laser shots } \\
\text { only }\end{array}$ & $\begin{array}{l}\text { Optics Performance/ } \\
\text { Conditioning }\end{array}$ & & & 138 & 138 \\
\hline \multirow[t]{2}{*}{$(578=55 \%)$} & Laser Performance & & & 204 & 204 \\
\hline & Laser Calibration & & & 236 & 236 \\
\hline Total & & 152 & 37 & 855 & 1044 \\
\hline
\end{tabular}

j The scientific and engineering requirements for the National Ignition Facility (NIF) were established and documented in NIF FR\&PCs. In this document, NIF-0001006-OE (September 2006), the mission-related requirements and goals, as defined in the Justification of Mission Need, were translated into specific laser requirements such as laser energy, power, and a variety of beam characteristics. Capabilities for conducting experiments supporting the requirements of users with diverse needs and top-level operability, safety, and environmental requirements were also defined to ensure that, when completed, NIF would be operated in a manner consistent with its role as a national resource.

${ }^{k}$ Contained within Appendices D and F in the NIC EP Rev. 4 is the NIC PCC and the PC\&FRs, respectively. The NIC PCC and PC\&FRs represent the top-level system requirements that must be achieved to support the NIC objectives and to ensure that operations meet applicable federal, state, and local requirements for the protection of workers, the public, and the environment. 


\section{National Ignition Campaign Program Completion Report}

\section{References}

1. NA-10 Defense Programs Program Management Manual, U.S. Department of Energy, Washington, D.C., NA13-PMM-04-001 (December 30, 2004), later revised, NA14-PMM-080001, Revision 1 (January 12, 2010).

2. NIF Ignition, JASON Report [JSP-05-340], Mitre Corporation, July 2005.

3. National Ignition Campaign Execution Plan, in UCRL-AR-213718, NIF-0111975-AA, Rev. 0 (July 2005). Later revised: Revision 4.0, NIF-0111975-AE (September 2010 signed by NNSA in January 2011).

4. National Ignition Facility Governance Plan (Version 0), LLNL-AR-416565, NIF-0115829-AA (September 28, 2012). Approved by NNSA October 1, 2012. 


\section{National Ignition Campaign Program Completion Report}

\section{B. Milestones}

The NIC Schedule Baseline by high level WBS shown below is from Appendix C of the NIC EP Rev. 4.0.

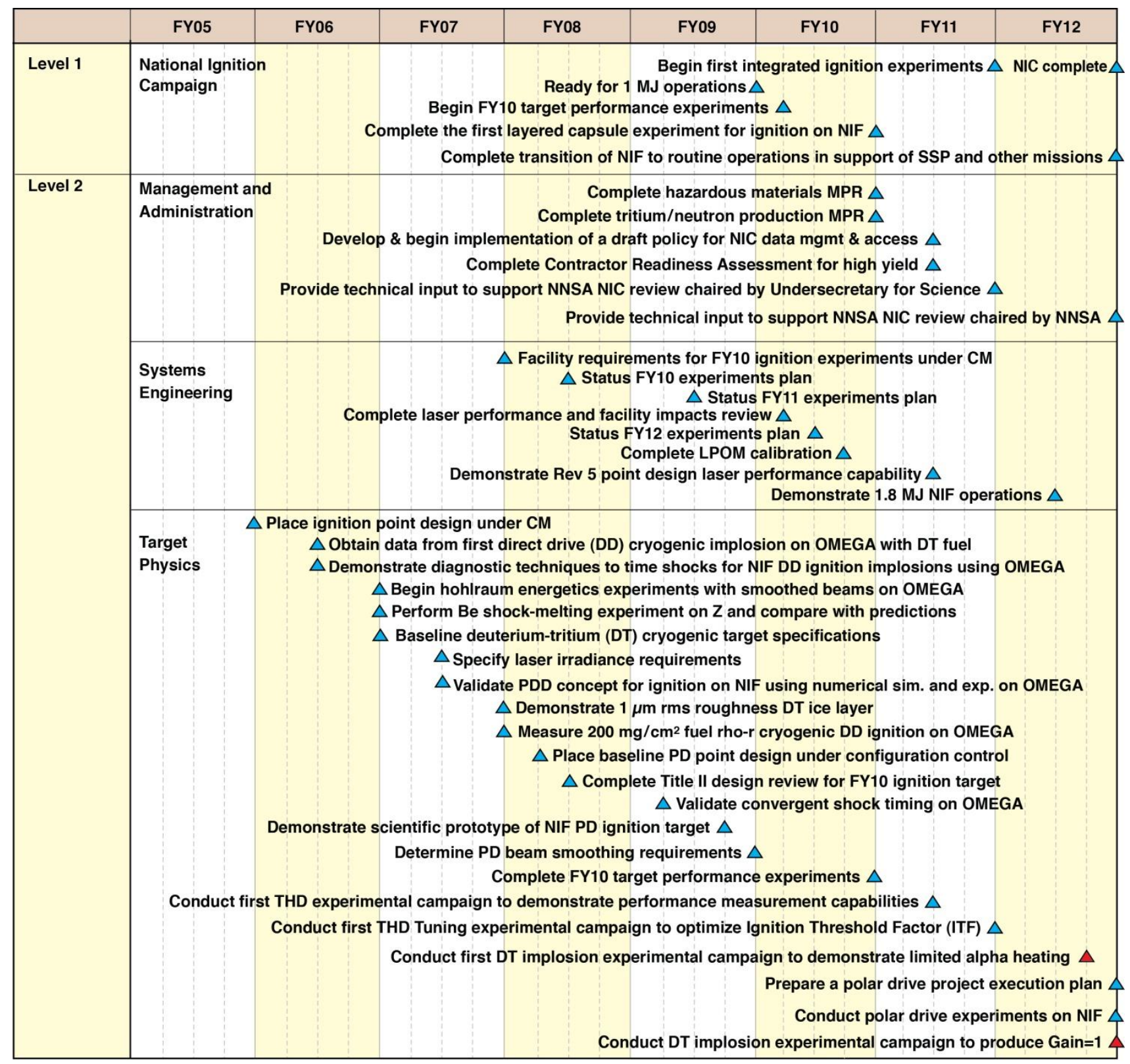

NIF-0505-10900s2r80L1 


\section{National Ignition Campaign Program Completion Report}

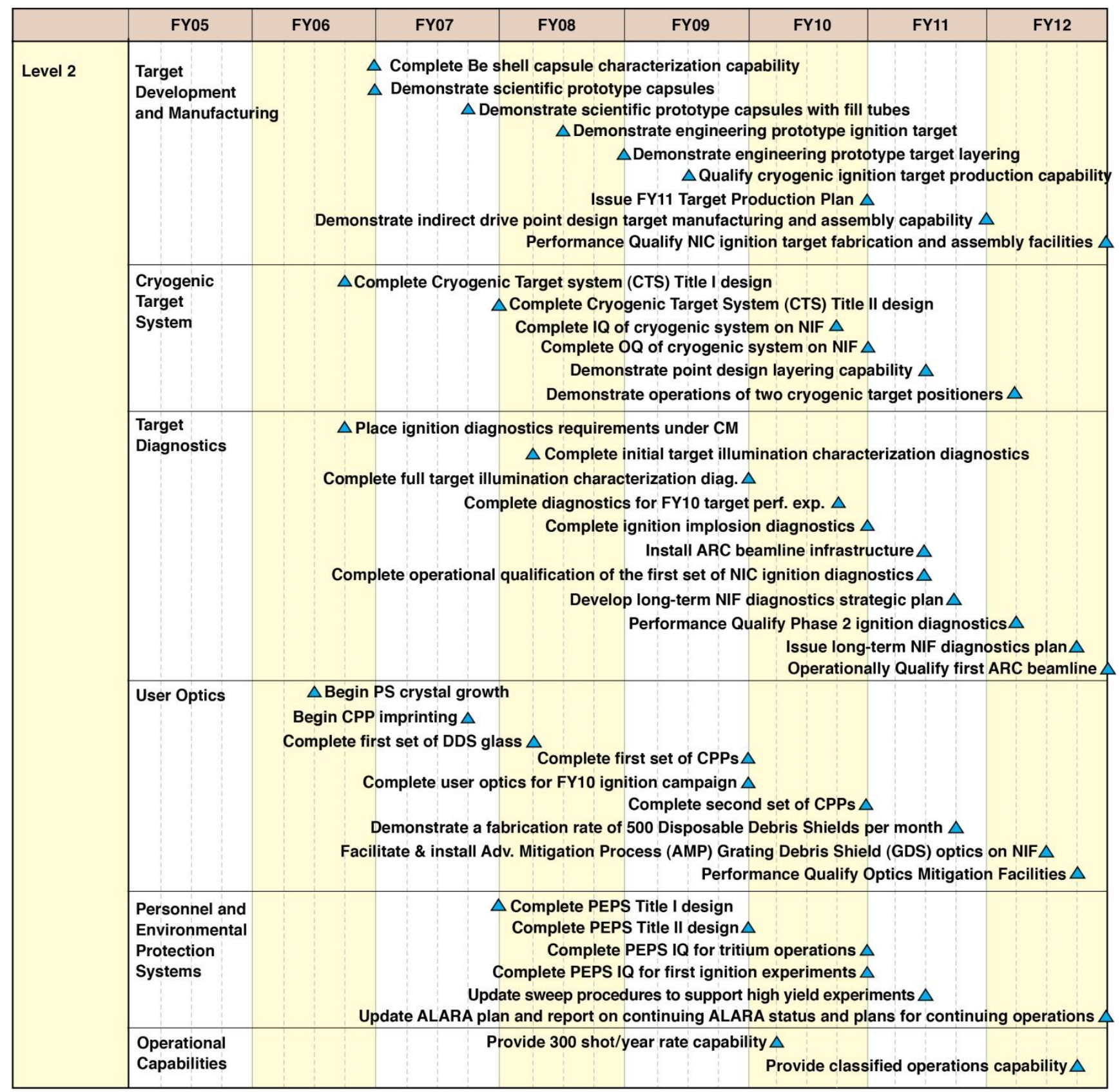

NIF-0505-10900s2r80L2 


\section{National Ignition Campaign Program Completion Report}

\section{Work Breakdown Structure}

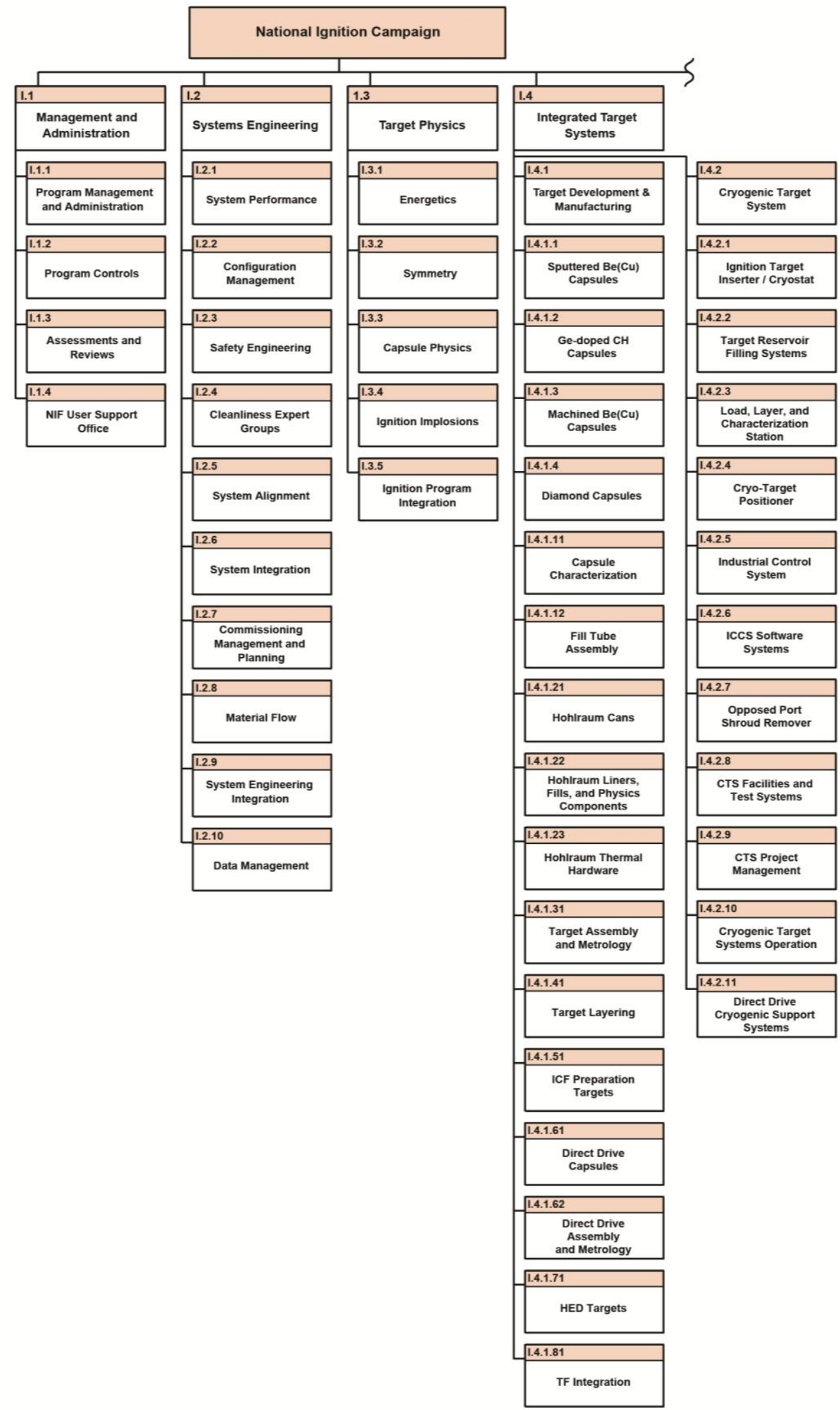




\section{National Ignition Campaign Program Completion Report}

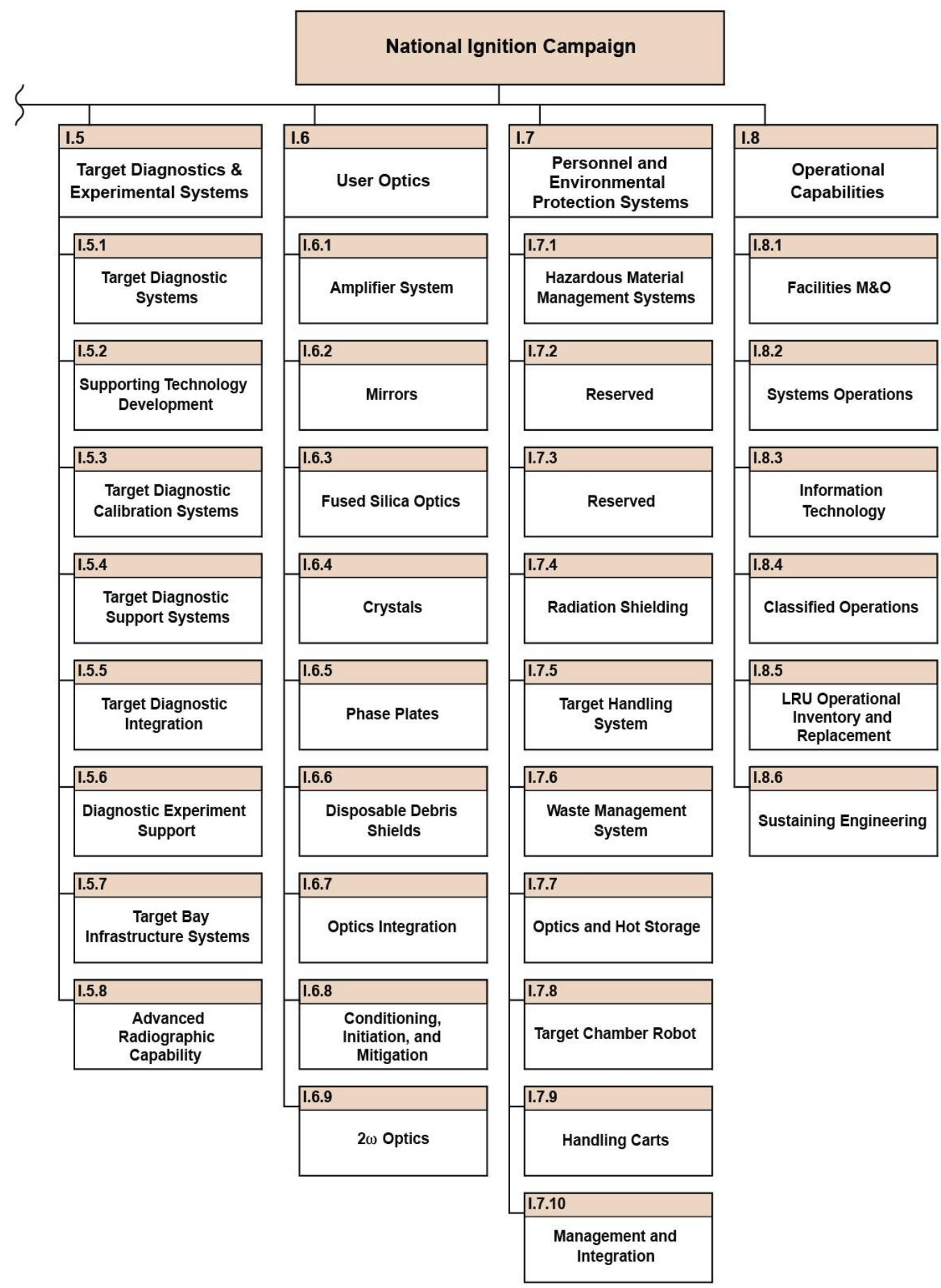




\section{National Ignition Campaign Program Completion Report}

\section{Project Completion Criteria}

The Project Completion Criteria by high level Work Breakdown Structure (WBS) shown below are from the Appendix D of the NIC EP Rev. 4.0.

\begin{tabular}{|c|c|}
\hline WBS & Completion Criteria \\
\hline I. 1 & $\begin{array}{l}\text { Complete Conduct of Operations } \\
\text { Complete NIF User Facility Guide }\end{array}$ \\
\hline $\mathrm{I} .2$ & Provide LPOM for multi-bundle shot setup \\
\hline $\mathrm{I} .3$ & $\begin{array}{l}\text { Design and execute NIC experimental campaigns } \\
\text { Execute direct drive experiments and develop a plan for a polar drive program on } \\
\text { NIF }\end{array}$ \\
\hline I.4.1 & Provide targets that meet NIC experimental plan requirements \\
\hline I.4.2 & Provide capability to field layered ignition targets \\
\hline I.5 & $\begin{array}{l}\text { Provide a core set of optical, } x \text {-ray, neutron, and radiographic diagnostics sufficient to } \\
\text { support initial ignition, HED, and other user applications during routine facility } \\
\text { operations }\end{array}$ \\
\hline I.6 & $\begin{array}{l}\text { Provide continuous phase plates (CPPs) that are required for the ignition experiments } \\
\text { and the manufacturing capability to develop and fabricate additional CPPs }\end{array}$ \\
\hline I. 7 & $\begin{array}{l}\text { Provide the personnel and environmental protection systems necessary for routine } \\
\text { operations including yield (Tritium Processing System [TPS], shield doors, } \\
\text { Hazardous Material Management Area [HMMA], and radiation monitoring system) }\end{array}$ \\
\hline I.8 & $\begin{array}{l}\text { Provide the capability to demonstrate the NIF Functional Requirements and Primary } \\
\text { Criteria } \\
\text { Provide an initial ignition platform and operational capabilities to support post-NIC } \\
\text { SSP experiments, including ignition application experiments }\end{array}$ \\
\hline
\end{tabular}




\section{National Ignition Campaign Program Completion Report}

\section{E. Funding Summary}

The NIC Funding Baseline by major technical effort (MTE) and participating site shown below is from Appendix B of the NIC EP Rev. 4.0.

\begin{tabular}{|c|c|c|c|c|c|c|c|c|}
\hline Site & MTE & FY06 & FY07 & FY08 & FY09 & FY10 & FY11 & FY12 \\
\hline $\mathrm{GA}$ & 10.7 & 15,756 & 17,580 & 18,726 & 18,170 & 17,822 & 15,655 & 16,056 \\
\hline \multicolumn{2}{|c|}{ GA Total } & 15,756 & 17,580 & 18,726 & 18,170 & 17,822 & 15,655 & 16,056 \\
\hline \multirow[t]{4}{*}{ LANL } & 10.1 & 12,161 & 11,698 & 12,633 & 12,514 & 15,000 & 13,253 & 13,252 \\
\hline & 10.2 & & & & & & & \\
\hline & 10.3 & & & & 289 & & 0 & \\
\hline & 10.7 & 420 & & & & & & \\
\hline \multicolumn{2}{|c|}{ LANL Total } & 12,581 & 11,698 & 12,633 & 12,803 & 15,000 & 13,253 & 13,252 \\
\hline \multirow[t]{4}{*}{ LLE } & 10.1 & 26,327 & 25,723 & 29,703 & 29,415 & 32,799 & 35,888 & 36,606 \\
\hline & 10.2 & & & & & & & \\
\hline & 10.3 & & 592 & 596 & 1,019 & 130 & 0 & \\
\hline & 10.7 & 12,306 & 12,332 & 22,801 & 21,241 & 21,166 & 21,589 & 22,021 \\
\hline \multicolumn{2}{|c|}{ LLE Total } & 38,633 & 38,647 & 53,100 & 51,675 & 54,095 & 57,477 & 58,627 \\
\hline \multirow[t]{5}{*}{ LLNL } & 10.1 & 35,585 & 40,265 & 58,744 & 56,760 & 57,846 & 59,201 & 59,200 \\
\hline & 10.2 & & & & & & & \\
\hline & 10.3 & 41,881 & 44,737 & 66,447 & 64,753 & 71,972 & 77,070 & 73,828 \\
\hline & 10.5 & & & & & 421 & & \\
\hline & 10.7 & 7,804 & 15,413 & 32,730 & 109,324 & 169,760 & 156,476 & 159,720 \\
\hline \multicolumn{2}{|c|}{ LLNL Total } & 85,270 & 100,415 & 157,921 & 230,837 & 299,999 & 292,747 & 292,748 \\
\hline \multirow[t]{2}{*}{$\overline{S N L}$} & 10.1 & 535 & 500 & 783 & 994 & 1,089 & 1,164 & 1,164 \\
\hline & 10.3 & 530 & 630 & 396 & 140 & 150 & 75 & 76 \\
\hline \multicolumn{2}{|c|}{ SNL Total } & 1,065 & 1,130 & 1,179 & 1,134 & 1,239 & 1,239 & 1,240 \\
\hline \multicolumn{2}{|c|}{ Grand Total } & 153,305 & 169.470 & 243.559 & 314.619 & 388,155 & 380.371 & 381,923 \\
\hline
\end{tabular}

\begin{tabular}{|c|c|c|c|c|c|c|c|c|}
\hline MTE & \begin{tabular}{|l} 
Site \\
\end{tabular} & FY06 & FY07 & FY08 & FY09 & FY10 & FY11 & FY12 \\
\hline \multirow[t]{4}{*}{10.1 Ignition } & LANL & 12,161 & 11,698 & 12,633 & 12,514 & 15,000 & 13,253 & 13,252 \\
\hline & LLE & 26,327 & 25,723 & 29,703 & 29,415 & 32,799 & 35,888 & 36,606 \\
\hline & LLNL & 35,585 & 40,265 & 58,744 & 56,760 & 57,846 & 59,201 & 59,200 \\
\hline & SNL & 535 & 500 & 783 & 994 & 1,089 & 1,164 & 1,164 \\
\hline \multicolumn{2}{|l|}{10.1 Total } & 74,608 & 78,186 & 101,863 & 99,683 & 106,734 & 109,506 & 110,222 \\
\hline \multirow[t]{5}{*}{ 10.3 Diagnostics, Cryo, and Support } & LANL & & & & 289 & & 0 & \\
\hline & LLE & & 592 & 596 & 1,019 & 130 & 0 & \\
\hline & LLNL & 41,881 & 44,737 & 63,447 & 64,753 & 71,972 & 77,070 & 73,828 \\
\hline & SNL & 530 & 630 & 396 & 140 & 150 & 75 & 76 \\
\hline & NTS & & & 3,000 & & & & \\
\hline \multicolumn{2}{|l|}{ 10.3 Total } & 42,411 & 45,959 & 67,439 & 66,201 & 72,252 & 77,145 & 73,904 \\
\hline 10.5 Joint Program & LLNL & & & & & 421 & & \\
\hline \multicolumn{2}{|l|}{10.5 Total } & & & & & 421 & & \\
\hline \multirow[t]{4}{*}{ 10.7 Facility Ops and Target Prod. } & GA & 15,756 & 17,580 & 18,726 & 18,170 & 17,822 & 15,655 & 16,056 \\
\hline & LANL & 420 & & & & & & \\
\hline & LLE & 12,306 & 12,332 & 22,801 & 21,241 & 21,166 & 21,589 & 22,021 \\
\hline & LLNL & 7,804 & 15,413 & 32,730 & 109,324 & 169,760 & 156,476 & 159,720 \\
\hline \multirow{2}{*}{\multicolumn{2}{|c|}{ 10.7 Total }} & 36,286 & 45,325 & 74,257 & 148,735 & 208,748 & 193,720 & 197,797 \\
\hline & & 153.305 & 169.470 & 243.559 & 314.619 & 388.155 & 380.371 & 381.92 \\
\hline
\end{tabular}




\section{National Ignition Campaign Program Completion Report}

\section{F. Participant Scope Summaries and Key Management Personnel}

\section{Participant Scope Summaries}

The Participant Scope Summaries shown below are from Appendix J of the NIC EP Rev. 4.0.

General Atomics: GA, an ICF target fabrication contractor, is a major player in all aspects of the integrated target system for the NIC. GA, with others, will develop the techniques for the fabrication and characterization of the ignition capsule and the cryogenic ignition hohlraum. Working with LLNL, GA will support target development and fabrication and cryogenic fielding of the NIC targets. Working with other sites, GA will support the manufacture and assembly of targets for supporting experiments on the other facilities.

Los Alamos National Laboratory: LANL will continue to use its core capabilities to make major contributions to six principal areas: laser-matter interaction, hohlraum and ablator development, ablator characterization and testing, capsule fabrication research, ignition target design, and advanced fusion diagnostics. These areas were chosen because of their synergism with LANL core capabilities and other high energy density science programs and because they have strong prospects for risk mitigation in the NIC program.

Lawrence Livermore National Laboratory: LLNL will have overall responsibility for implementing, managing and coordinating the NIC Execution Plan and NIC activities including providing procedures, processes, and systems for controlling/maintaining the NIC technical, cost, and schedule baseline and for managing work at LLNL. Specific responsibilities will include operating the NIF, providing systems engineering/campaign management activities, maintaining the ignition point design, leading the collaboration on integrated target systems and target diagnostics, providing user optics and the operating inventory, and providing the personnel and environmental protections systems.

Sandia National Laboratories: SNL will contribute staff, Z/ZR shots, and associated experimental equipment and targets to support four major task areas of the National Ignition Campaign: designing, testing, and developing a manufacturing plan for the NIC cryogenic target x-ray shield system; participating in mitigating the $\mathrm{x}$-ray generated debris and electromagnetic pulse (EMP) on NIF diagnostics; conducting experiments on Z/ZR to validate computational models of the melting of shocked beryllium and other capsule materials and continuing to collaborate on ablator and hohlraum experiments on OMEGA; and participating in developing radiographic techniques using short-pulse lasers.

University of Rochester, Laboratory for Laser Energetics: LLE has taken a major role in the NIC by supporting indirect-drive ignition and is leading the national direct-drive ICF effort, including Polar-Drive (PD) experiments on the NIF. LLE will lead development of shock timing diagnostics and the shock timing campaign on the NIF, perform OMEGA hohlraum energetics campaigns, and participate in the development of NIF nuclear and other diagnostics. LLE will also provide OMEGA diagnostic development/calibration shots and will leverage its cryogenic experience by developing multi-axis viewing of cryogenic targets in hohlraums and studying layering physics. LLE will operate OMEGA and OMEGA Extended Performance in support of NIC experiments. 


\section{National Ignition Campaign Program Completion Report}

\section{Management Personnel}

The key management personnel in the NIC and the conclusion of the program are listed in Table 2.

Table 2. Key NIC management personnel by organization.

\begin{tabular}{|l|l|l|}
\hline Organization & Title & Name \\
\hline NNSA & Director, Office of Inertial Confinement Fusion & Jeffery Quintenz \\
\hline NNSA & NIC Manager & Kirk Levedahl \\
\hline LLNL & NIC Director & Edward Moses \\
\hline LLE & NIC Principal Deputy Director & Steven Loucks \\
\hline LLNL & NIC Deputy Director for Operations & Patricia Baisden \\
\hline GA & NIC Institutional Deputy Director & Joseph Kilkenny \\
\hline LANL & NIC Institutional Deputy Director & Mary Hockaday \\
\hline LLNL & NIC Institutional Deputy Director & Patricia Baisden \\
\hline SNL & NIC Institutional Deputy Director & Keith Matzen \\
\hline LLE & NIC Institutional Deputy Director & Steven Loucks \\
\hline
\end{tabular}




\section{SUMMARY OF KEY NIC ACCOMPLISHMENTS BY WBS I.1-Management and Administration}

The main scope of work conducted under the NIC WBS Element I.1, Management and Administration, is to provide the management, administration, and oversight along with the tools, processes, and procedures necessary to execute the NIC within the performance, cost, and schedule baseline.

\section{A. NIC Execution}

The NIC Execution Plan (NIC EP Rev 4.0) states that the NIC Director is responsible for implementing the NIC and providing overall management of all NIC activities. During the NIC, the NIC Director established the work scope, schedule, and cost baseline, coordinating NIC-related activities with the participating partner sites. The project management tool used for financial planning and tracking the NIC program baseline was the NIF/NIC Planning System (NPS), the same financial tool used for the NIF Project. This earned value management system, shown in the left-hand portion of Figure 1-1, provided the capability to plan work packages and provide data reports by organization, institution (partner site), WBS, subproject, and MTE. The NPS was used to monitor the NIC baseline plan, including actual and planned costs, and the status of earned value activities as defined by individual control account plans. The integrated cost performance data across all partner sites, along with scope, schedule, technical highlights, and milestone status by each NIC WBS element, was provided to NNSA on a monthly basis through a formal written progress report (see the right-hand portion of Figure 1-1).

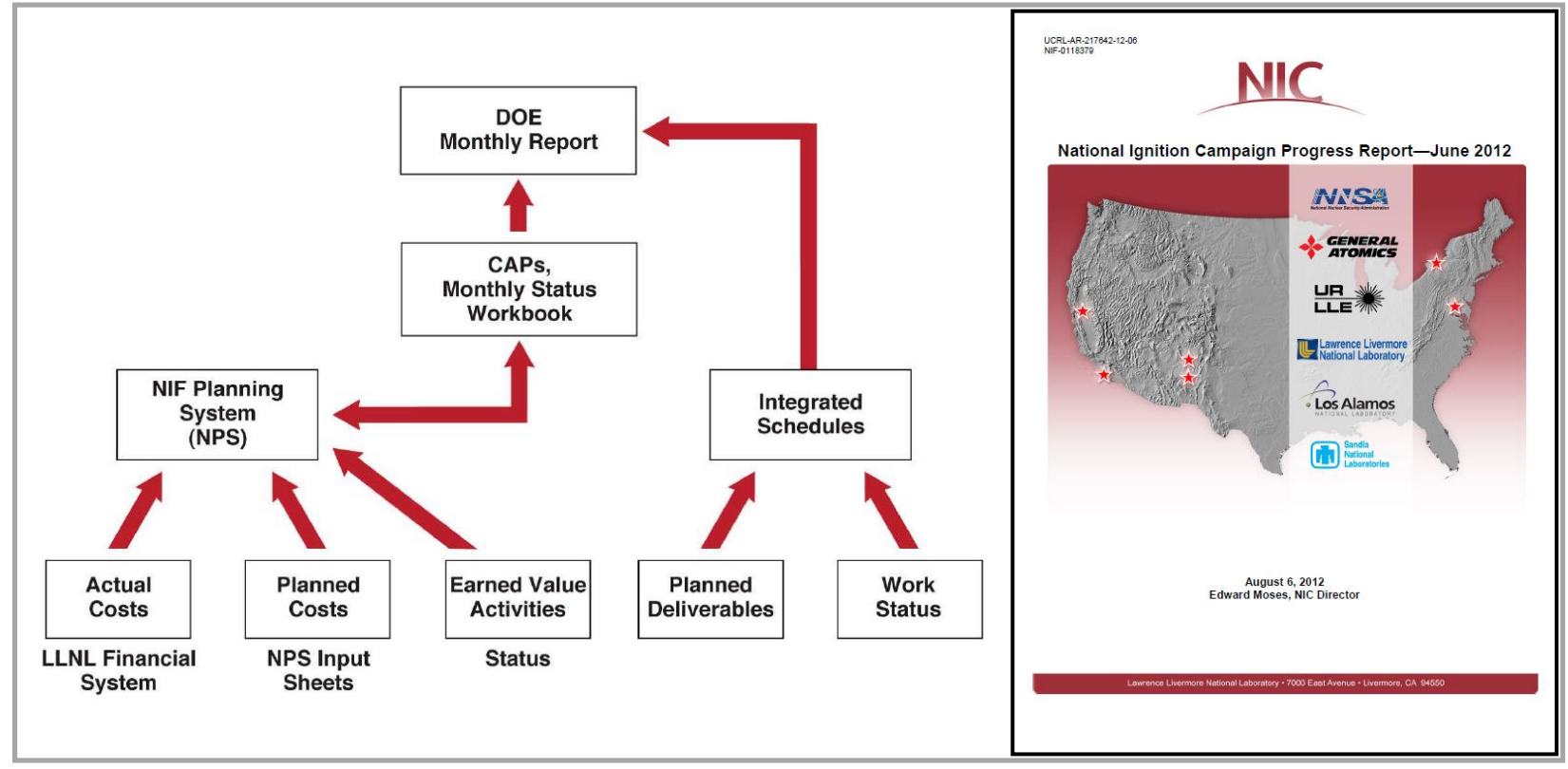

Figure 1-1. (Left) Diagram of the NIF Planning System, the financial planning and tracking tool used by NIF. (Right) Picture of the cover of the NIC Monthly Progress Report.

A significant part of the management and administration effort for the NIC program involved communication with all stakeholders, NNSA's SSP (NA-11), the Office of Inertial Confinement Fusion (NA-112), the NIC Federal Program Manager, and the NIC partner sites. Program progress was continuously monitored and documented in a weekly NIF shot report that was disseminated widely. In addition, program progress and milestone status was provided via weekly teleconferences with the NIC Institutional Deputies, biweekly teleconferences with NNSA, and detailed input on NIC program progress for NNSA's Quarterly Inertial Confinement Fusion Program Progress Report to Congress. Also reported were NIC monthly cost data provided via the approved processes, quarterly milestone status updates 


\section{National Ignition Campaign Program Completion Report}

reported through the Milestone Reporting Tool, and milestone completions documented in formal milestone completion letters to NNSA.

The NIC actively participated in a series of technical reviews dating back to the first JASON review in 2005 (and again in 2009). NIC also supported technical reviews conducted by the NIC Technical Review Committee $^{1}$ (December 2009, February 2011, and May 2012), quarterly reviews chaired by then Under Secretary Steve Koonin (October 2010, January 2011, June 2011, and October 2011), and ICF program updates chartered by NNSA (January 2012 and May 2012) (see Figure 1-2). In addition, the NIC experimental plan and results were extensively assessed during a 2-week period (July-August) in 2011 by a group of 17 recognized experts drawn from the national and international scientific ICF community and again as part of the Science of Fusion Ignition on NIF Workshop (May 2012). Pertinent documents, presentations, and review reports from all reviews were made available to the NIC partner sites and NNSA through controlled, password-protected websites. Program progress was communicated to the scientific community through presentations at national and international conferences. In particular, NIC results were significant contributions to the programs at the annual American Physical Society Division of Plasma Physics meetings and the international Inertial Fusion Science and Applications (IFSA) conferences in 2007, 2009, and 2011.

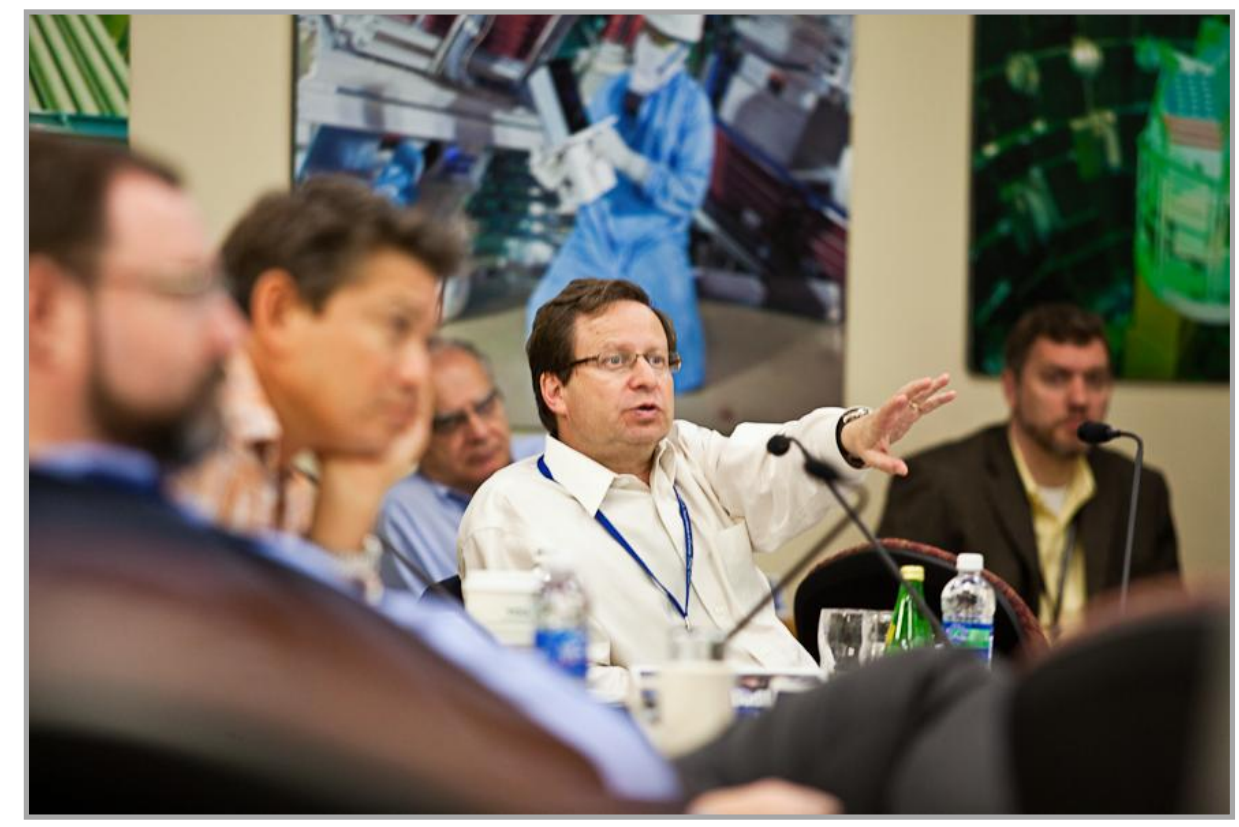

Figure 1-2. Under Secretary Steve Koonin chairs October 2010 NIC review.

Many of the activities required to successfully complete the NIC involved the development and implementation of new capabilities (hardware, software, processes, and procedures). As such, it was essential that the execution of the NIC integrate quality assurance, security, Integrated Safety Management System principles, and applicable Environment, Safety, and Health requirements. This was an important management function that was executed to ensure personnel safety and proper stewardship of NIF as a national resource and key component of NNSA's Stockpile Stewardship Program.

NIC management was committed to providing a safe working environment at all participating sites. Maintaining a focus on continual improvement in occupational health and safety performance while protecting workers and the public, and preventing property loss or damage, was a priority. Accident and

${ }^{1}$ Committee established at the request of NNSA by Brig. Gen. Garrety Harencak in 2009 and chaired by Dr. Alvin Trivelpiece, Director Emeritus of the Oak Ridge National Laboratory. 


\section{National Ignition Campaign Program Completion Report}

injury preventive measures, including well-designed qualification and training programs and worker health awareness campaigns, were put into place. Evidence of this commitment to safety was reflected in the Total Recordable Case (TRC) rate, tracked monthly in the NIC Monthly Progress Report. ${ }^{\mathrm{m}}$

\section{B. NIF User/Visitor Office}

The NIF User/Visitor Office was established in 2008 to facilitate establishing governance for NIF as a national user facility beginning in October 2013. This office also serves as the primary point of contact for NIF researchers regarding policies and procedures associated with NIF use and access to NIF and related LLNL facilities.

A significant activity carried out by the NIF User/Visitor Office was to develop the NIF Governance Plan (see Figure 1-3) [1], associated policies for facility use and data access, and the processes for allocating facility time and developing the annual NIF multi-mission integrated facility use plan. This effort also included developing proposal submission and review processes to ensure that the highest quality science experiments are conducted on NIF. The NIF User/Visitor Office serves as the focal point for developing and building the fundamental science user community on NIF. The User/Visitor Office initiated a NIF User Group, hosts annual User Group meetings and other activities that foster collaborations with other NIF researchers, solicits and assists in the review of proposals, and for successful proposals, works with the principal investigators to ensure scheduling and implementation of their experiments on NIF.

The first proposal solicitation for fundamental science experiments at the NIF was issued in late 2009. Forty proposals for NIF facility time were received and reviewed by the Science on NIF Technical Review Committee (TRC), chaired by Professor Robert Rosner of the University of Chicago. The review was conducted via web conference, with all principal investigators

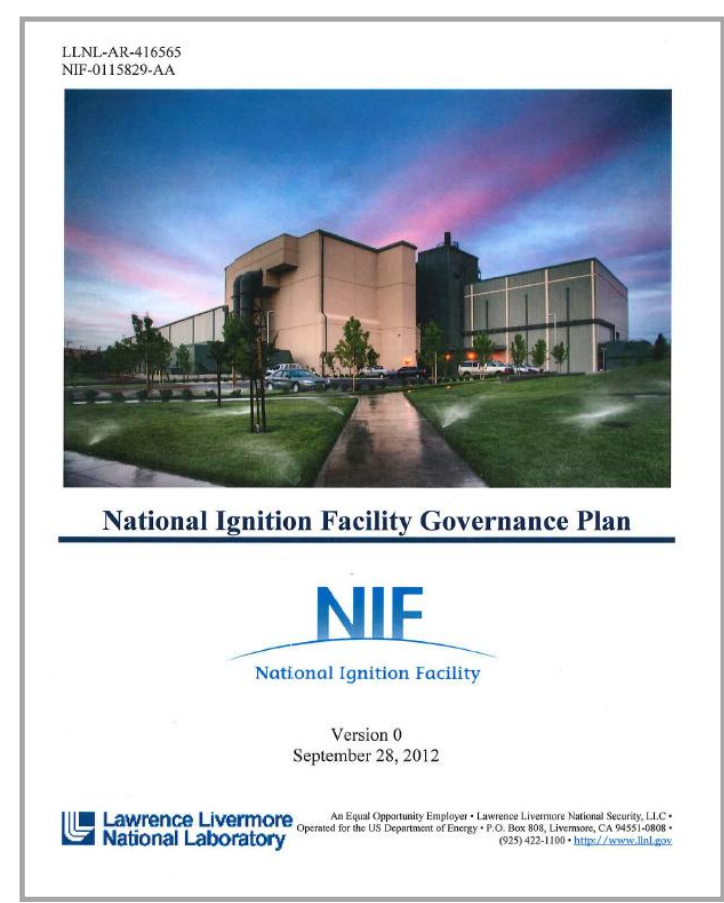

Figure 1-3. The NIF Governance Plan, which was completed in September 2012. participating, and resulted in eight experiments being recommended for execution. Prior to implementation of the Science on NIF TRC, two fundamental science proposals were reviewed and approved, and execution of both research efforts was initiated during NIC. They were:

- Radiation effects on Rayleigh-Taylor (RT) instability growth using ultrahigh-temperature hohlraums (a collaboration between University of Michigan and LLNL), and;

- Iron and carbon equation of state (EOS) studies (a collaboration between UC Berkeley, Princeton University, and LLNL).

Two of the proposals recommended by the Science on NIF TRC were also initiated during NICproduction of low-energy neutrons $(<100 \mathrm{keV}$ ) for nucleosynthesis (a collaboration between LLNL, LANL, University of Oslo in Norway, CEA, Ohio University, Themba Laboratory in South Africa, and Grenoble Institute of Technology in France) and carbon EOS in the gigabar pressure regime (a

${ }^{\mathrm{m}}$ The TRC base target was 3.1, while the stretch target was 1.8. The NIC program at LLNL consistently maintained a TRC rate well below the stretch target objective; the rate at the end of NIC at LLNL was 0.7. 


\section{National Ignition Campaign Program Completion Report}

collaboration between UC Berkeley, LLNL, University of Jena in Germany, Carnegie Institution of Washington, SLAC National Accelerator Laboratory, GSI Helmholtz Center for Heavy Ion Research in Germany, University of Rostock in Germany, Imperial College London in the UK, UC Los Angeles, and AWE).

Concurrent with the fundamental science proposal solicitation, a concept development competition was held for fundamental science experiments at NIF. This competition also received 40 applications, including a number of highly innovative ideas for NIF fundamental science experiments judged to have the potential to be submitted as proposals in future facility time competitions. Fourteen groups were each provided a monetary award to further develop their ideas; the average award was $\$ 70 \mathrm{~K}$. Final reports from the funded groups are expected by the end of 2012.

The NIF User Group was initially formed in 2010 with Professor Justin Wark (University of Oxford) as interim chair of the User Group Executive Committee. The first major onsite meeting of the User Group, shown in Figure 1-4, was held at LLNL on February 12-14, 2011, and featured over 160 attendees, of which approximately 115 were from institutions other than LLNL. Fifteen countries were represented, and over 30 students and postdoctoral fellows attended. The meeting included a wide range of technical talks describing NIF experiments and related work. Since the User Group meeting, a set of bylaws were developed and an election was held for the leadership of the User Group. The currently elected members of the Executive Committee hail from seven institutions in three nations.

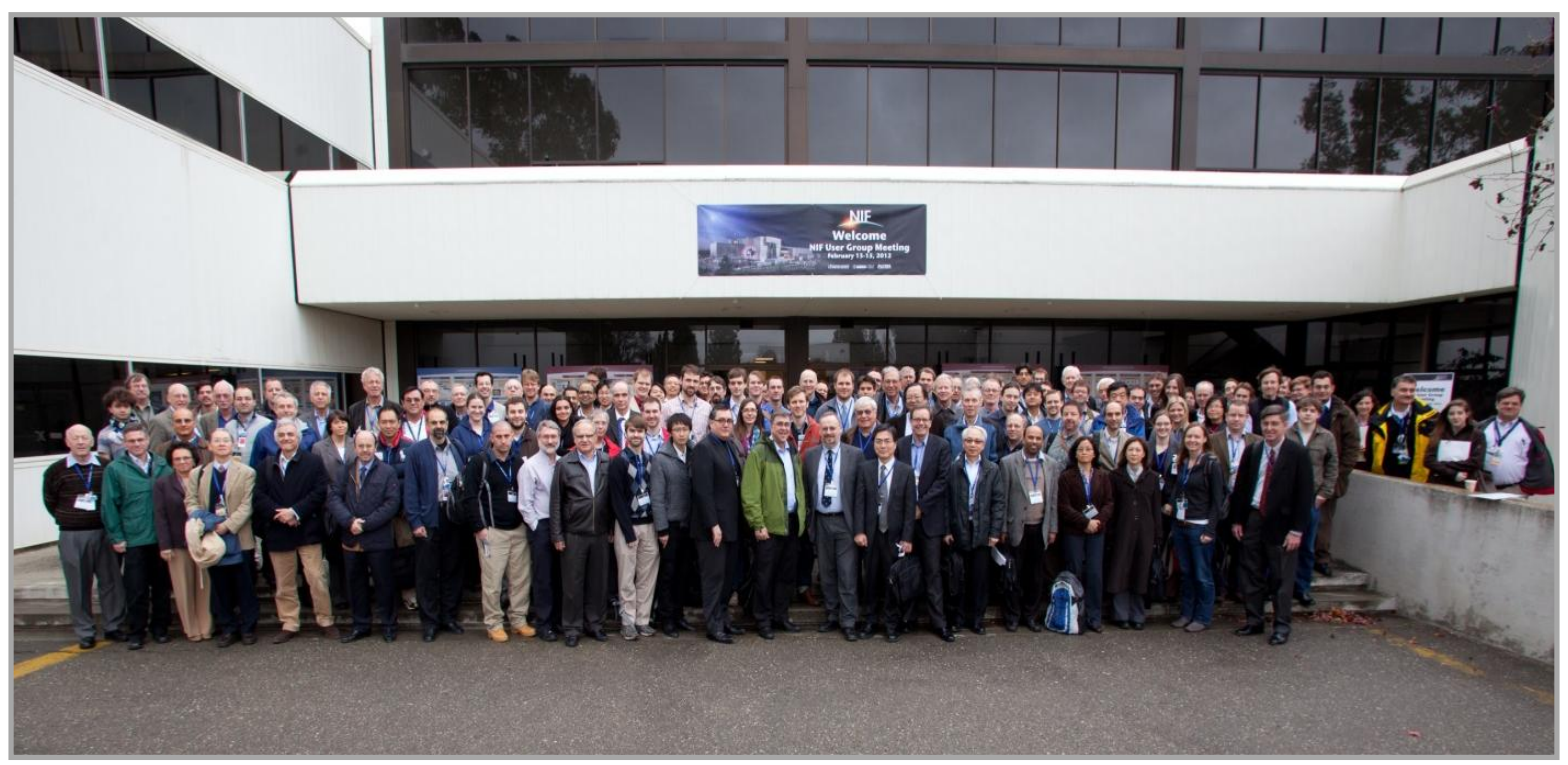

Figure 1-4. Attendees at NIF User Group meeting, Feb. 12-15, 2011.

Day-to-day efforts of the NIF User/Visitor Office focus on infrastructure and administrative support to NIF users/visitors, including badging; operational, security, and safety training; office and laboratory space; website management; information technology support; shot request form preparation assistance; and NIF User Guide maintenance. The office also supports frequent or longer-term visiting experimentalists, researchers, and subcontractors involved with the NIF and other related programs. The NIF User/Visitor Office provides assistance to LLNL hosts and their visitors to help them navigate through processes associated with extended visits such as work authorization, training, and on-site computer access. At any given time, the NIF User/Visitor Office maintains the status and provides assistance to approximately 100-150 visiting researchers.

The NIF User/Visitor Office also develops general policies and procedures related to the use of NIF. The Office worked closely with the NIC to create a NIC data policy. Similar policies are being developed and implemented for SSP, fundamental science, and other experiments planned for FY2013 and beyond. 


\section{National Ignition Campaign Program Completion Report}

The NIF User/Visitor Office is located at LLNL within the HED campus. The HED campus, which includes Building 481 and portions of Buildings 381 and Trailer 3724, provides office and meeting space for the scientists, engineers, and other personnel from the NIF and Photon Science, Weapons and Complex Integration, and Physical and Life Sciences directorates engaged in NIF target design and experimental planning and execution. Co-location of these individuals in the HED campus integrates the NIF effort and enhances the collaboration between LLNL scientists and visiting researchers engaged in NIF-related activities. The HED campus is shown in Figure 1-5.

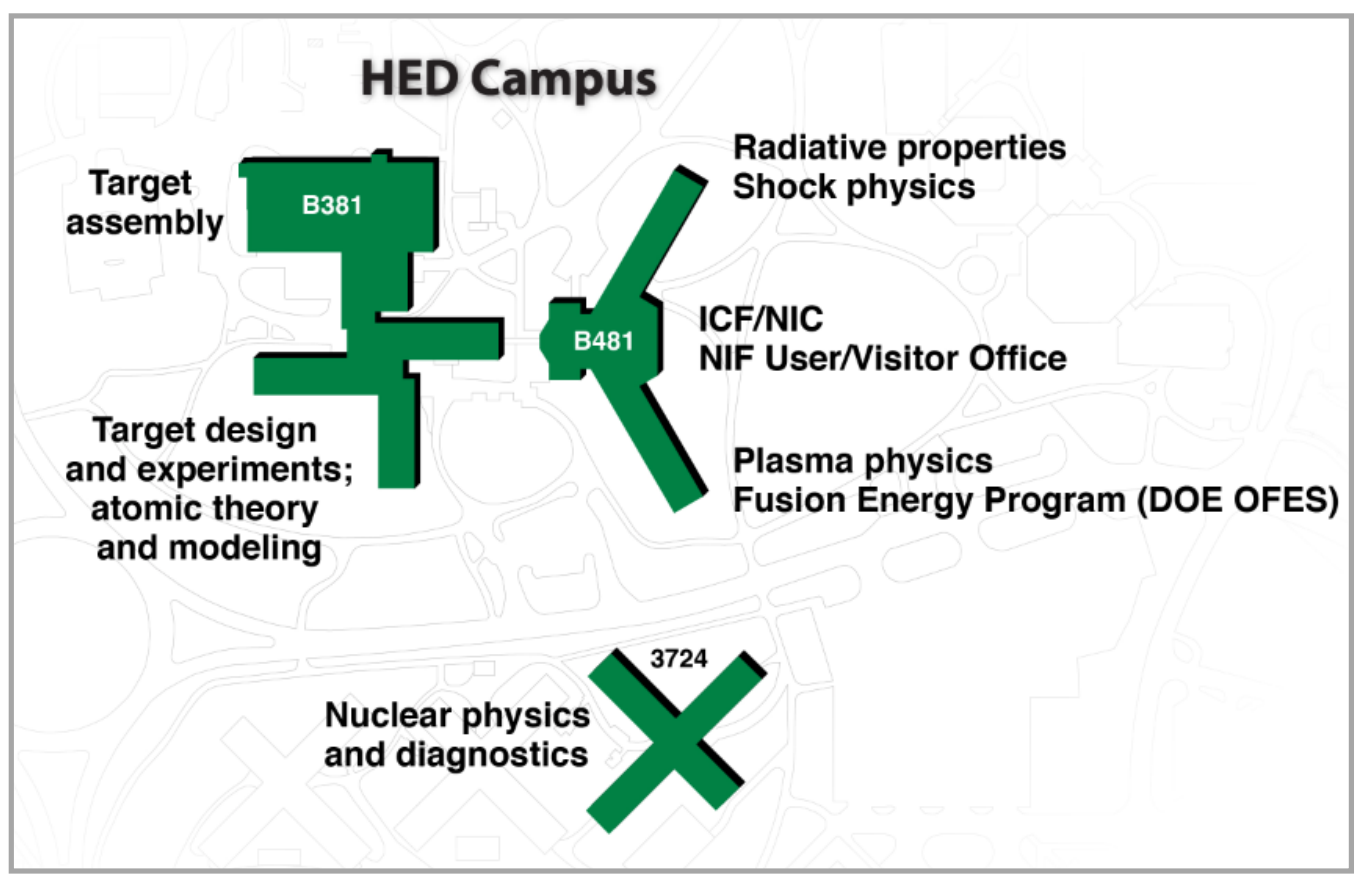

Figure 1-5. Diagram of the LLNL HED campus.

\section{References}

1. National Ignition Campaign Execution Plan, in UCRL-AR-213718, NIF-0111975-AA, Rev. 0 (July 2005). Later revised: Revision 4.0, NIF-0111975-AE (September 2010) signed by NNSA in January 2010. 


\section{National Ignition Campaign Program Completion Report}

\section{I.2-SYSTEMS ENGINEERING}

The main scope of work conducted under the NIC WBS Element I.2, Systems Engineering, is to provide the capabilities necessary to support user experimental campaigns on NIF. This includes development of laser performance models to specify the laser and diagnostics configurations; formation and utilization of expert group reviews and analyses to ensure that the systems are operated within their allowable limits; and establishment of additional capabilities required to successfully conduct campaigns, such as configuration management, requirements management, experiment control, system integration, and user interfaces for data access.

\section{A. Shot Operations and Laser Performance}

The most important function of Systems Engineering is to support user experimental campaigns while ensuring that NIF operates effectively (satisfying the requirements of the user), efficiently (maximizing the utilization of NIF across all missions), and safely (protecting personnel, the environment, and the NIF laser and all subsystems by maintaining the highest safety standards during operations). A wide variety of processes, procedures, and tools have been developed to satisfy these needs.

\section{NIF Operations Loop}

NIF Operations Loop integrates all the tasks required to execute a systems shot on NIF. The Loop ensures that predefined laser performance configurations are appropriately matched with each desired shot, and that similar shots are scheduled in a manner that minimizes reconfiguration time. It also includes checkpoints to confirm that the selected laser configuration will safely meet shot goals. Through the Loop, new capabilities are incorporated into a new or existing laser configuration, as appropriate, before configuring the laser for a shot.

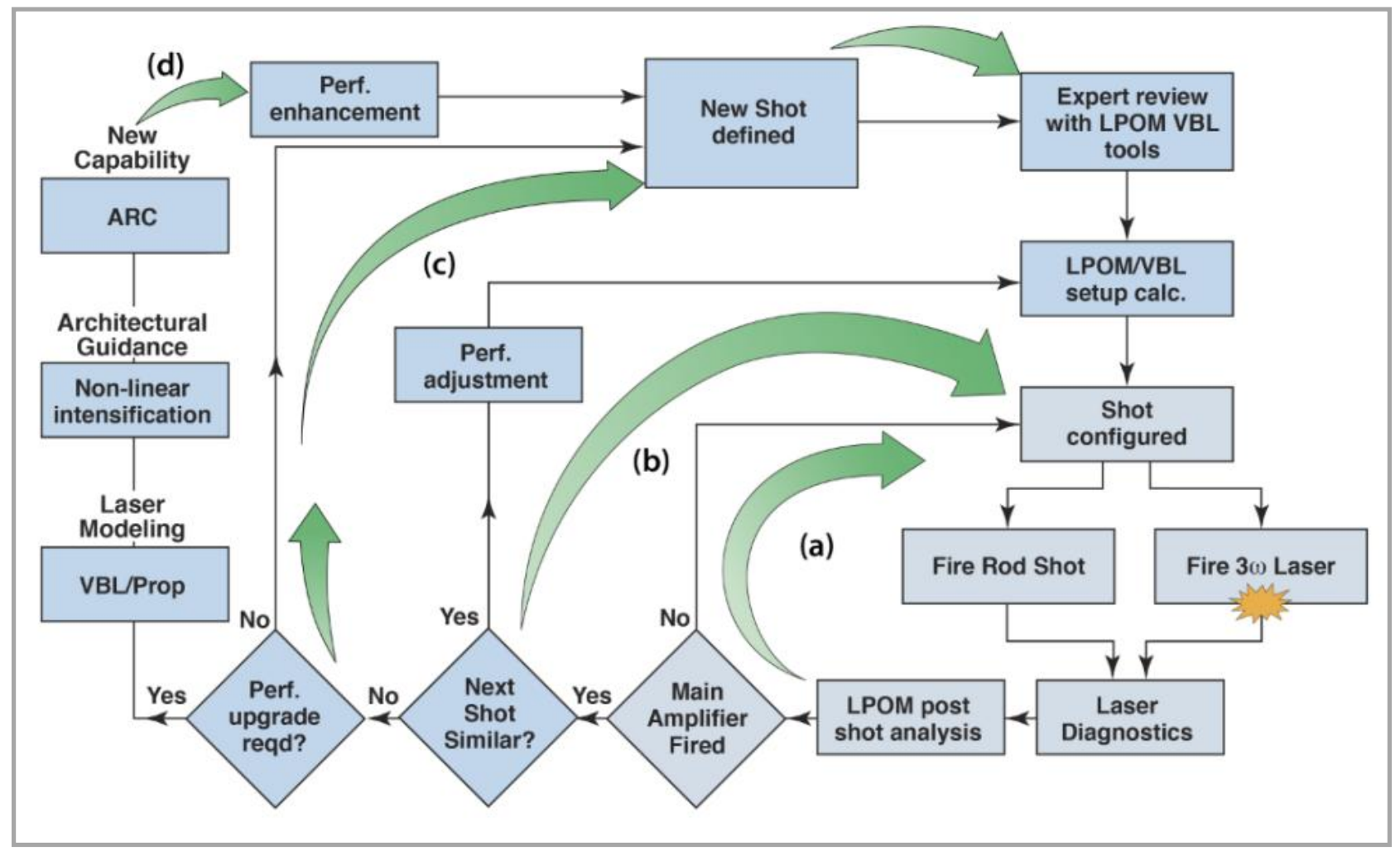

Figure 2-1. NIF Operations Loop illustrates the flow of shot execution tasks at NIF.

Figure 2-1 is a schematic illustrating the process for setting up NIF to execute laser shots. All the nested loops of activities flow through the act of firing the laser at bottom right in the figure. 


\section{National Ignition Campaign Program Completion Report}

a. At bottom right are the actions of configuring the laser for a shot, executing a full system shot or a low-energy rod shot, ${ }^{\mathrm{n}}$ and analyzing the laser performance data. Those actions are shown as part of a loop that operates on a timescale of minutes to hours.

b. A second, wider loop operating on a timescale of hours to days illustrates setup and execution of additional shots of a similar type, incorporating performance adjustments based on previous shots.

c. A third, wider loop operating on a timescale of days shows the process of setting up and performing expert review for a different shot type.

d. Finally, the outermost loop shows the path for laser experiments that require significant performance enhancement or extension of current capabilities to new areas of performance. This loop operates on a timescale of weeks to months.

All of these operations paths rely on our ability to model and hence predict the performance of the NIF laser and have that model respond to observed performance of the laser on previous shots. Thus, a key part of the operations loop is LPOM, described in the next section.

\section{Laser Performance Operations Model}

Success on many of the NIF laser's missions depends on obtaining precisely specified energy waveforms from each of the 192 beams over a wide variety of pulse lengths and temporal shapes, with precise energy balance among the beams. Since the energetic performance of each of the beams is different, due to slight differences in amplifier gains and optical transmission losses in the constructed beamline, a computational model of the facility is necessary to accurately determine required input conditions and to generate the requested output. LPOM [1-3] has been developed to provide this function for NIF. LPOM is operated from the control room, where it communicates with a software supervisor, integrating it directly with the NIF Integrated Computer Control System (ICCS). In addition to supplying shot setup information, the LPOM helps protect NIF equipment and archives shot data and analysis for future study. The LPOM may also be run in an off-line mode (not in the control room) for pre-shot setup and target design studies.

\section{a. LPOM Shot Setup}

As part of the setup process, LPOM maintains a current description of the system that includes the optical paths, optical losses, amplifier configurations, and frequency conversion configurations for each beam, as well as a database of diagnostic measurements, laser energy, and power at various locations along the beamline (see Figure 2-2).

A key component of LPOM is a detailed energy extraction and propagation code called Virtual Beam Line (VBL) [4, 5], the mainstay of design, verification, and component selection for the NIF laser system. VBL calculates the energetics (energy and temporal shape [power]) throughout the beamlines, based on the current optical properties of each of the NIF beamlines, to self-consistently determine the laser settings that will produce the required output temporal pulse. This four-dimensional (three spatial and one temporal) code models energy extraction from amplifiers by solving the Frantz-Nodvik equation [6], while propagation of the laser pulse is modeled using a Fast-Fourier Transform method (see Figure 2-3).

\footnotetext{
${ }^{\mathrm{n}}$ A rod shot is a laser shot (for which only the Injection Laser System amplifiers are energized), that amplifies the laser pulse up through the four-pass preamplifier only i.e. it does not fire the slab amplifiers.
} 


\section{National Ignition Campaign Program Completion Report}

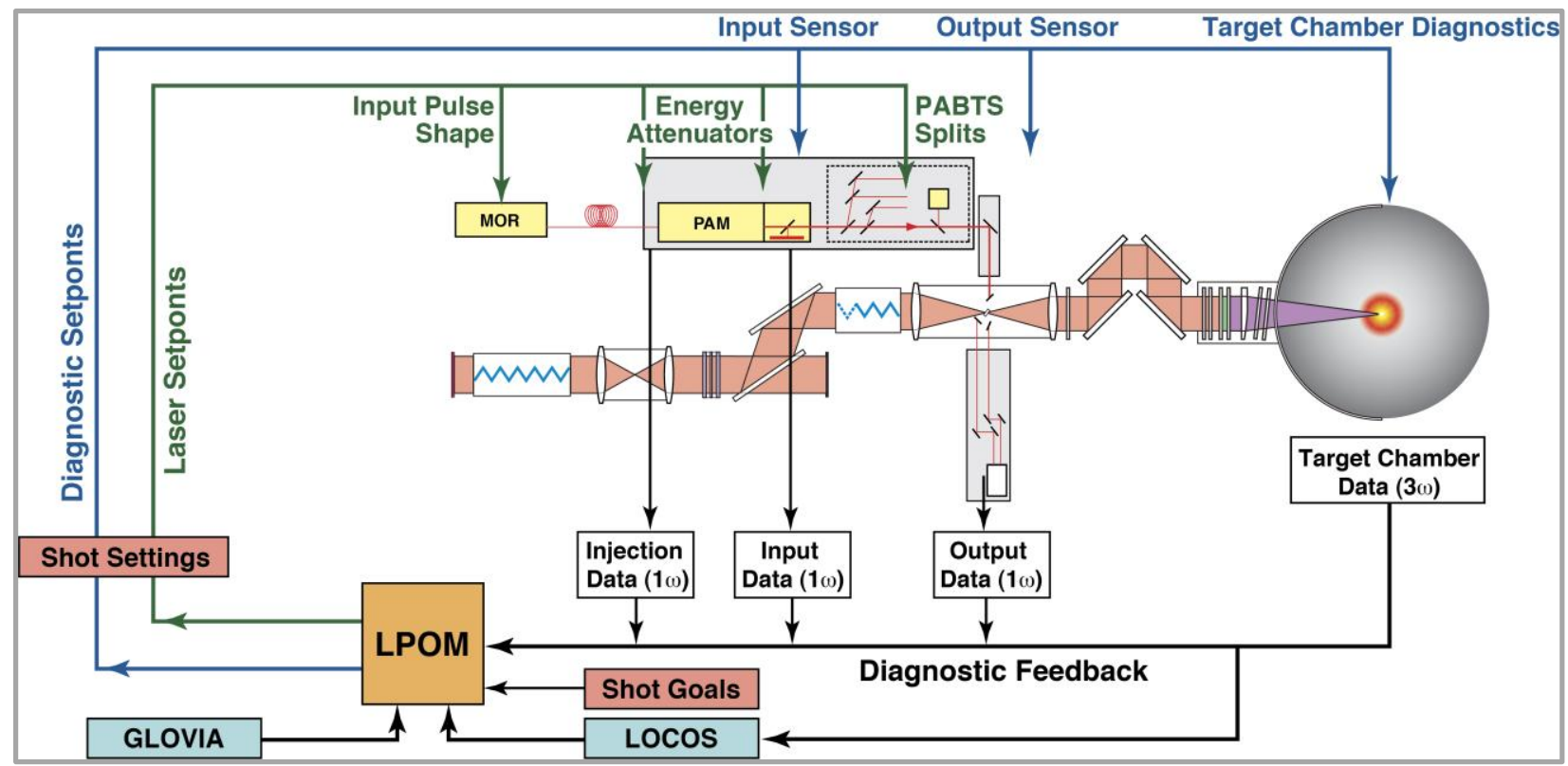

Figure 2-2. LPOM contains a detailed model of each beamline. Propagation calculations using the VBL model provide predicted energy and power throughout the laser, information that is used for equipment protection and to configure diagnostics via ICCS. Data results are presented in a web-browser format, with a series of linked web pages that provide successive levels of detail for each quad (group of four beamlines).

LPOM incorporates the slight optical imperfections that exist in various beamline components, and their effects, in the VBL propagation calculation either as measured metrology data or as representative powerspectral-density-based simulated phase screens. With this information, a quantitative prediction of the near-field beam modulation and far-field spot size can be made by LPOM. By maintaining an accurate description of the optical system, and by using a detailed physics model at its core, LPOM can precisely calculate the required settings for the Injection Laser System (ILS) that will produce the requested output energies and powers.

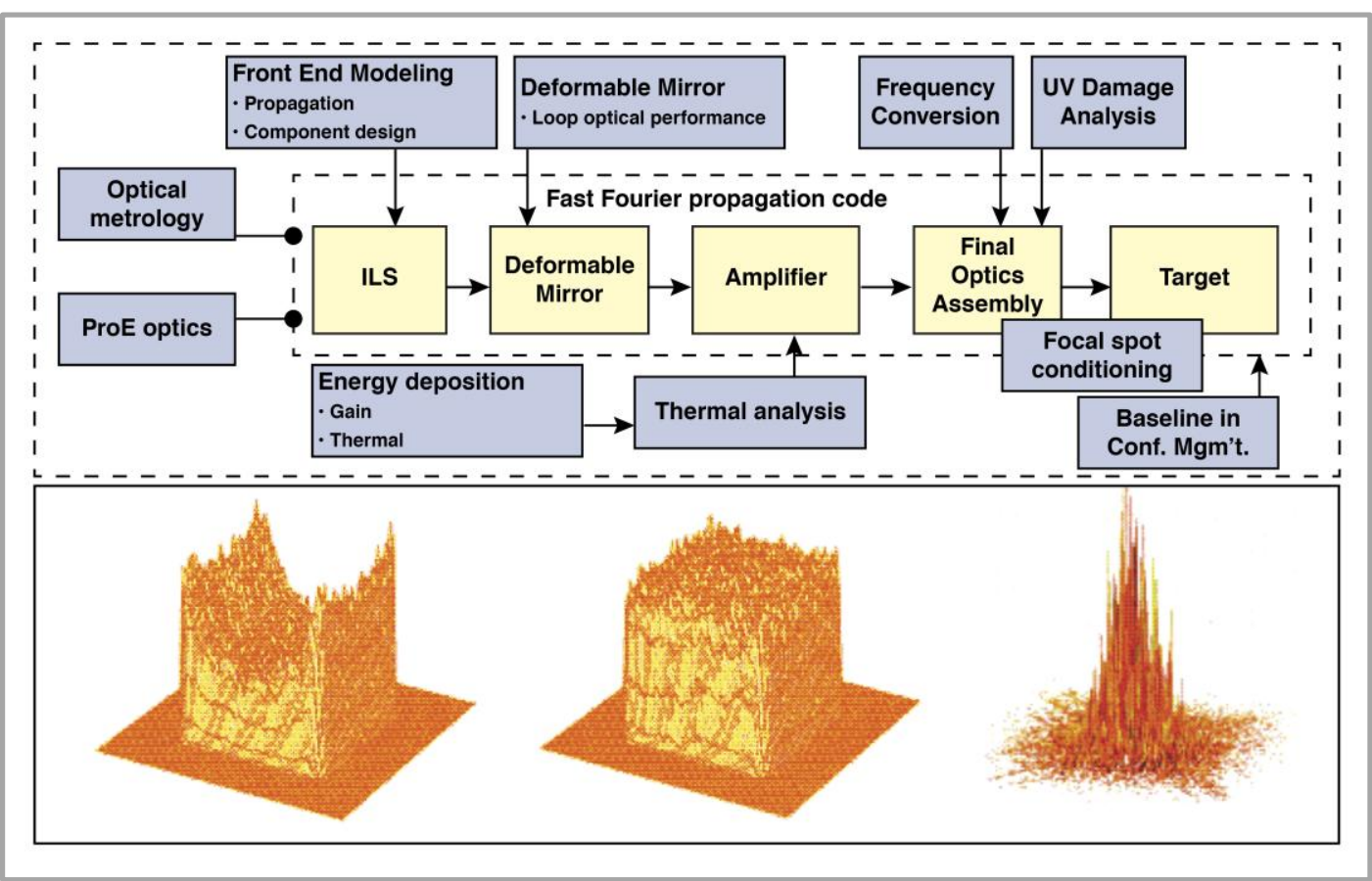

Figure 2-3. Using measured laser optics data sets, the Virtual Beam Line (VBL) code can model the desired laser configuration and calculate and analyze the fluence along the laser path for the entire laser system. 


\section{National Ignition Campaign Program Completion Report}

Upon completing its setup calculation, the LPOM has determined 1) the waveplate settings required by the splitting waveplates; 2) the attenuations for the input and output waveplates on the pre-amplifier module four-pass amplifier; and 3) the temporal pulse shape from the master oscillator. In addition, LPOM predicts the energy and power expected at each diagnostic location for the quad. These energies determine the attenuation settings necessary for each diagnostic device so that the shot is accurately measured. These settings are uploaded to the ICCS shot database, where they are accessed by the ILS and Laser Diagnostics subsystems during shot setup. At this point, all systems are ready to implement these settings, in preparation for a shot.

In order to maintain an accurate model of each beamline, LPOM requires feedback at the conclusion of each shot from each diagnostic. A suite of laser diagnostics provides data on the performance of NIF on each shot. The data from individual beams are used to determine the total power and energy, and the power and energy balance, on any given shot. When the predictions of the model begin to deviate from measured data, LPOM uses a set of measured data to modify or optimize its model of the laser. The LPOM therefore continually acquires shot data in order to update its energetics models (gains and losses in the main laser section, adjustable parameters for the frequency converter).

Real-time adjustments of the code's energetics parameters allows LPOM to predict total energies within $2 \%$, and provide energy balance within the four beamlines to within $2 \%$ for shots varying from 1 to $9 \mathrm{~kJ}$ (at a wavelength of $351 \mathrm{~nm}$ ) per beamline. In addition to the ILS settings, LPOM also predicts the energies and powers at each of the laser diagnostic locations in NIF, thereby ensuring that each diagnostic is configured to accurately measure the results of the shot.

\section{b. LPOM Equipment Protection}

A second function of LPOM is safeguarding NIF optics and works in concert with the Optics Recycling Loop (see Figure 2-4). After calculating the setpoints for a proposed shot, the LPOM provides a pre-shot assessment to the NIF Shot Director (SD) prior to the initiation of the shot. This assessment includes a report on the probability of a shot causing optical damage and the feasibility of achieving the proposed shot goals. LPOM employs optical damage models to assess the optics damage probability; this assessment is part of NIF's "defense-in-depth" approach to system protection, playing an administrative role in preventing conditions that could lead to unexpected optical damage. LPOM provides an additional equipment protection feature after a shot by verifying that the measured energies and powers match those predicted by the model.

LPOM's equipment protection module, designed to help minimize the risk of optical damage to the system, protects the ILS four-pass amplifier, the Preamplifier Beam Transport System optics, the main amplifier, and the Final Optics Assembly (FOA). The module has two components, both of which serve as administrative controls for equipment protection. The first part, called the Setup Assessment Code, checks the calculated system setup before the ICCS Laser Supervisory System implements the setup. The Shot Setup module uses the Shot Setup energetics calculations to evaluate the damage probability for key optical beamline elements. These tests include the probability of optical damage initiation, beam filamentation, and excessive beam spatial contrast. If all performance metrics are within prescribed limits, the system is declared ready. If either criteria (equipment protection or inability to meet the goals) is not satisfied, alerts are sent to the ICCS Laser Supervisory System and SD.

The second protection function, called the Setup Verification Module, evaluates the actual pulses generated during a series of low-energy shots prior to the initiation of a full-system shot; its role is to verify that the actual laser pulse and energy output from the ILS match predictions and will not damage the main laser or final optics. In addition, LPOM compares the measured near-field spatial profile to that predicted, and verifies that the energy splits among the beamlines of a quad (four similar beamlines) agrees with the requested setpoint. Using rules or guidelines provided by the NIF Program, the Setup Verification code (via the SD) can stop the shot cycle if there is a high probability of significant equipment damage or significant deviation from predicted results. If the measured energies are acceptable, the SD is alerted that the system is prepared for a primary shot. 


\section{National Ignition Campaign Program Completion Report}

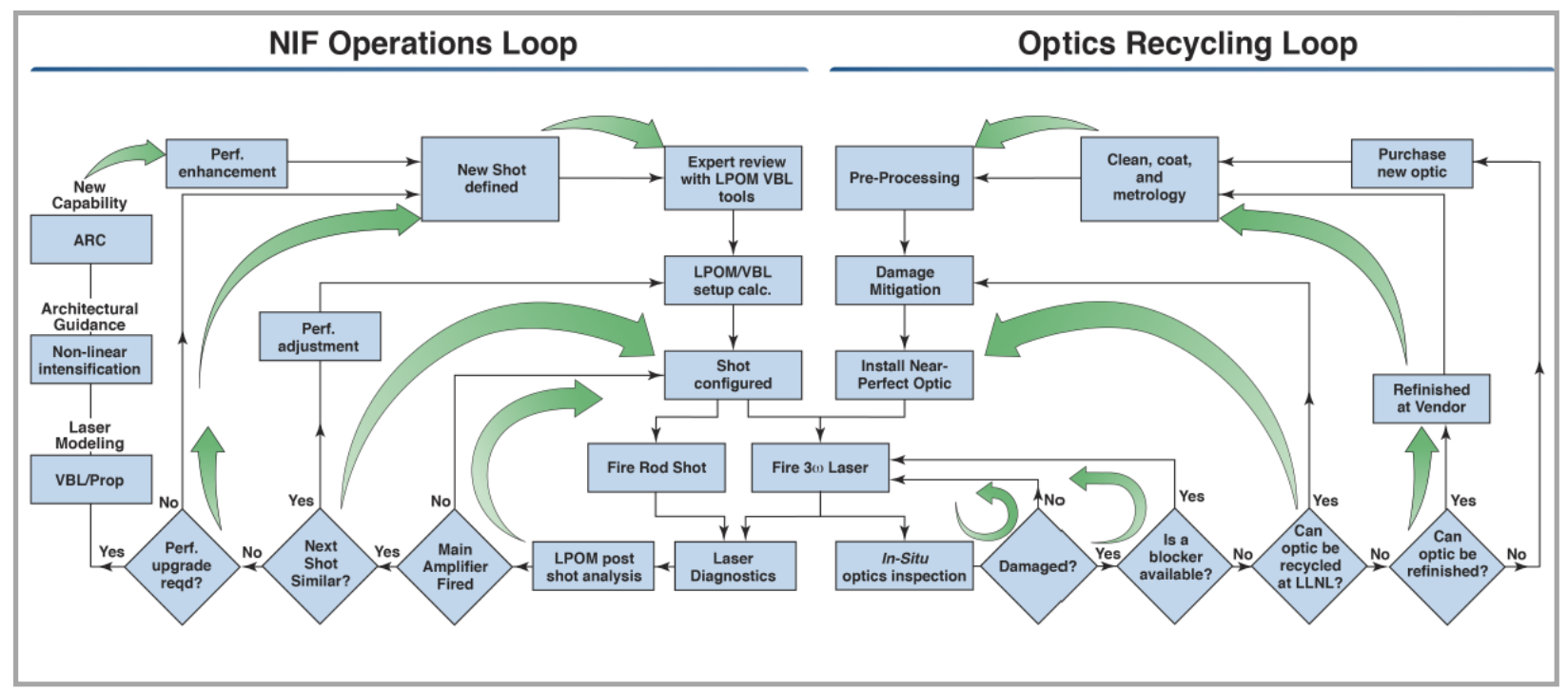

Figure 2-4. Within the NIF Operations Loop, LPOM works in concert with the Optics Recycling Loop described in I-6, User Optics, to ensure safe and cost-effective operations at NIF.

Figure 2-5 shows a screen capture of the shot verification screen on the LPOM graphical user interface (GUI) at the end of a low-energy shot. Metrics that fall inside or outside of prescribed limits are highlighted with green and red, as appropriate.

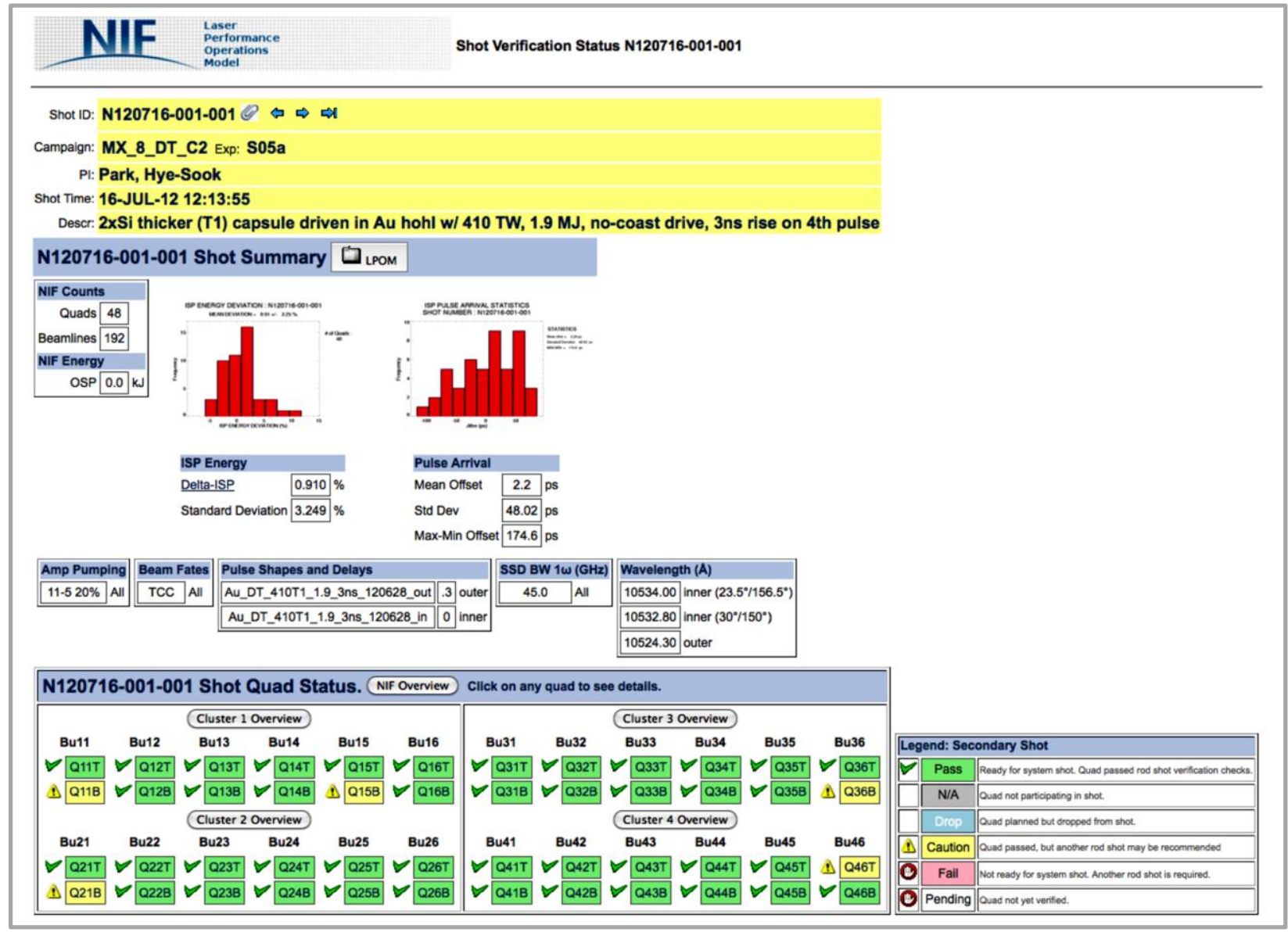

Figure 2-5. During calculation of laser setup, LPOM compares fluences and intensities throughout the beam line against operational limits to minimize instances of optical damage. In addition, during a laser shot cycle, LPOM analyzes laser data to verify that system is set up as required. 


\section{National Ignition Campaign Program Completion Report}

\section{c. LPOM Laser Performance Data Analysis and Reporting}

The final function of LPOM is performing post-shot data analysis and reporting laser performance. To do this, LPOM is directly linked to the ICCS shot database and upon request, it can quickly (within minutes) provide the NIF SD and/or the user with a report that compares predicted and measured results, summarizing how well the shot met the requested goals. In addition, the LPOM data reporting system can access and display near-field and far-field images taken on each of the laser diagnostic locations, and provide comparisons with predicted images. The results of the post-shot analysis are displayed on the LPOM GUI, while a subset of the analysis is presented to the SD through a Shot Supervisor interface. A data analysis screen from the LPOM GUI is shown in Figure 2-6.

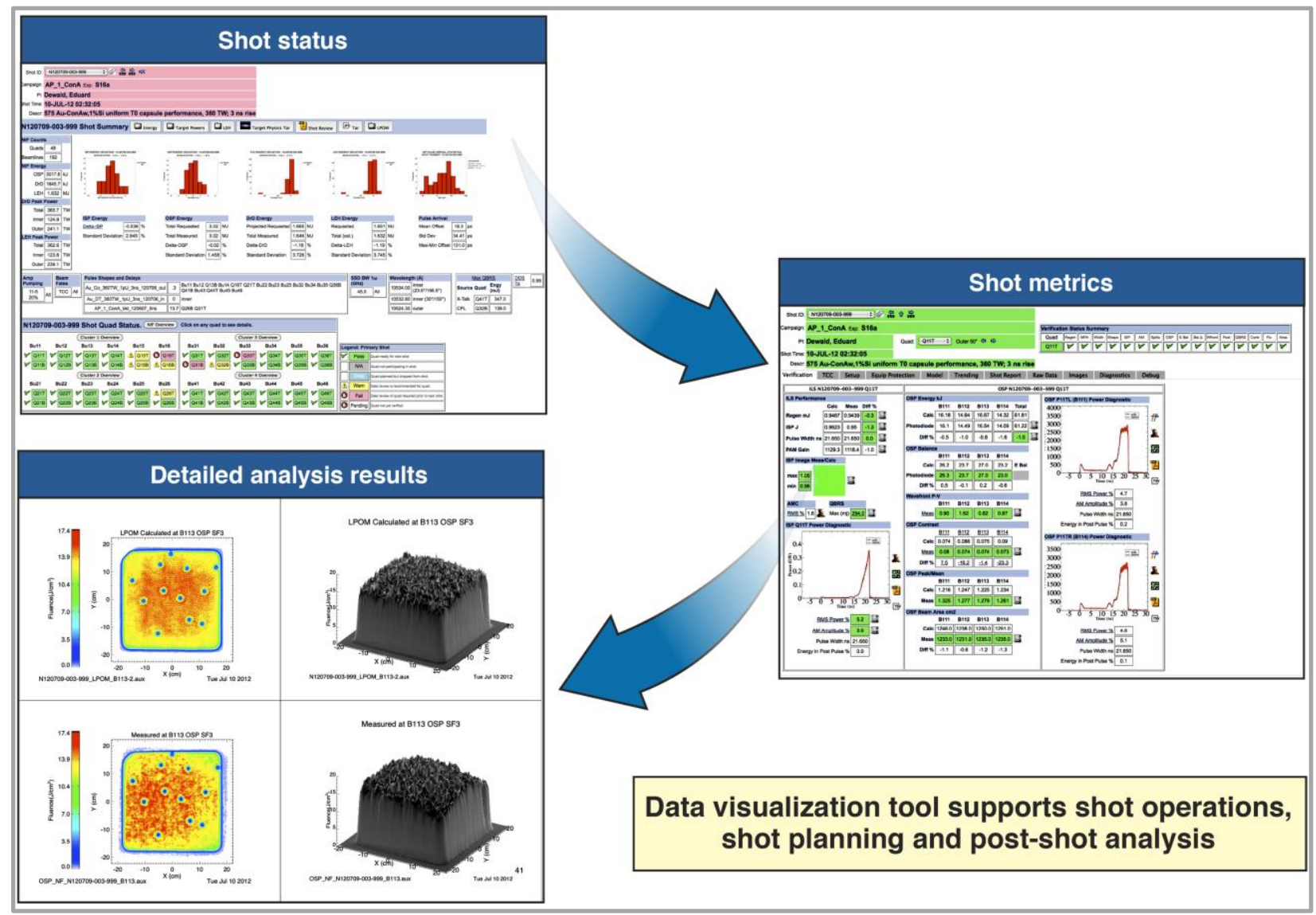

Figure 2-6. After each shot, a web-based format enables the detailed laser performance for any beamline to be examined using a suite of data trending and analysis tools.

\section{d. Demonstrated NIF Laser Performance}

Since the beginning of NIF commissioning in 2006, nearly 2000 system shots $^{\circ}$ have been performed. In particular, a large number of high-energy shots with output pulses at $1 \omega, 2 \omega$, and $3 \omega$ have been taken. During the course of these experiments, LPOM has thoroughly demonstrated its ability to accurately

o A system shot is a shot that uses the amplification of the main laser at the full $37 \mathrm{~cm}$ beam aperture to generate up to $\sim 26 \mathrm{~kJ}$ of energy per beam at the fundamental laser energy $(1 \omega)$. 


\section{National Ignition Campaign Program Completion Report}

model laser energetics and ensure that precision laser pulses to target and experiments are conducted safely.

The NIF frequency conversion system has been operated up to energies of $10 \mathrm{~kJ}$ per beamline, making it the highest energy $3 \omega$ laser system in the world. NIF displays excellent agreement between measured and specified $3 \omega$ power over a wide dynamic range of operating conditions. The robustness of the LPOM $1 \omega$ and $3 \omega$ models enables the system to accurately produce the highly-shaped $3 \omega$ temporal pulses required for ignition experiments, while meeting the extreme energy and power balance requirements placed upon NIF. In particular, LPOM was used to accurately model and operationally obtain the 500 terawatt level of energy on July 5, 2012, as shown in Figure 2-7. With LPOM, NIF delivers exquisite precision of user requests for power balance, pulse shape, energy, and power throughout the laser pulse.

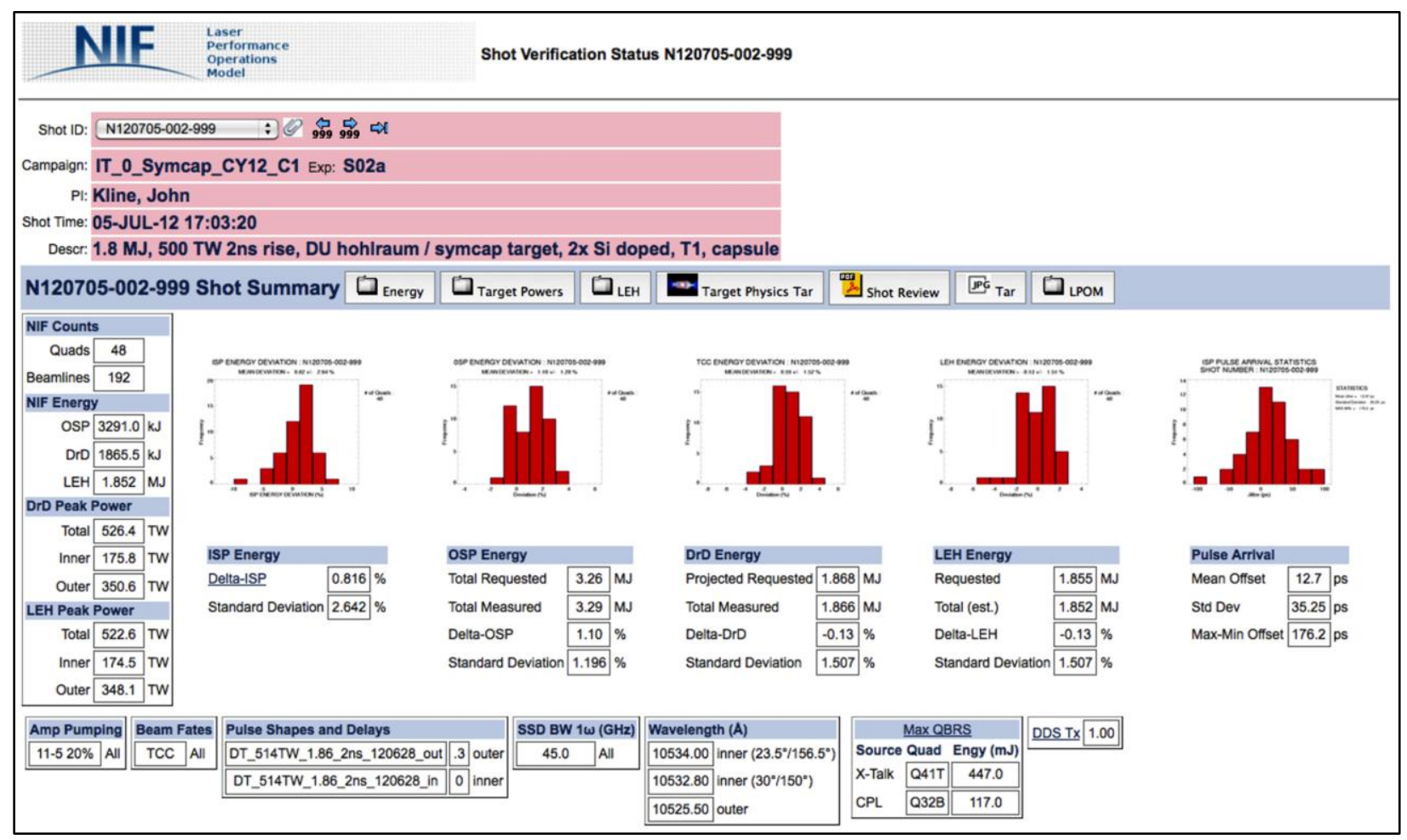

Figure 2-7. LPOM has been used to safely set up NIF to deliver 1.9 MJ (525 TW) in a 192-beam NIC shot, delivering within $0.13 \%$ of specified energy and quad-to-quad variation of $1.5 \%$. Fidelity of model allows LPOM to accurately set up NIF over the wide range of energies and powers required for the NIC campaign.

\section{B. NIF Information Technology Software Tools and Infrastructure}

LPOM is only one (albeit crucial) of the software and modeling tools used by Systems Engineering to support the NIF Operations Loop. These tools support all phases of a shot-from planning and scheduling an experiment, through setup, execution, data archiving, visualization, and analysis. The LPOM data are made available to users through a vast array of NIF Information Technology servers, networks, databases, and storage devices. A schematic diagram of functions provided by some of the software and modeling tools is shown in Figure 2-8. 


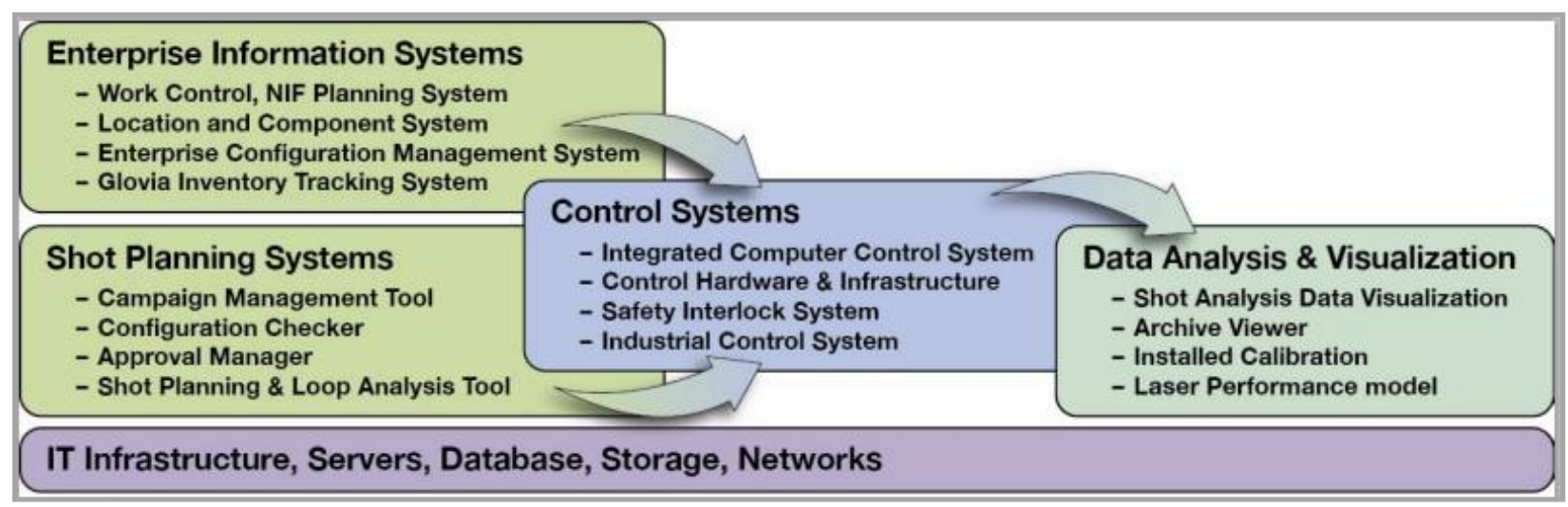

Figure 2-8. Shot data systems software supports pre-shot setup, calibration, shot execution, archiving, analysis, and visualization.

\section{Shot Planning, Facility Configuration, and Execution Tools}

Shot planning and scheduling is performed using the Shot Planning and Loop Analysis Tools. The laser and facility configuration for an experiment is generated with the Campaign Management Tool (CMT), a suite of software applications designed to translate experimental plans and specifications into actions for the control system.

Components of the CMT-managed automated shot cycle include:

- Inputting campaign shot goals from LPOM.

- Performing automatic alignment and wavefront correction.

- Configuring diagnostics and laser performance settings.

- Conducting countdown (4 min for software and $2 \mathrm{sec}$ for timing).

- Assessing shot outcome and archiving shot data.

A Shot Clock is provided as part of the suite of software tools so that the progression of the experiment can be monitored as the NIF control system implements the experimental parameters defined in the CMT.

\section{User Interfaces, Data Access, and Archiving}

NIF software tools have been designed and constructed to manage and integrate data from multiple sources, including machine state configurations and calibrations, experimental shot data and pre- and post-shot simulations. Data accumulation is on the order of 70 terabytes a year. During a shot, data from each diagnostic is automatically captured and archived into a database (see Figure 2-9). Arrival of new data triggers the Shot Analysis, Visualization, and Infrastructure (SAVI) engine, the first automated analysis system of its kind, which distributes the signal and image processing tasks to a Linux cluster and launches an analysis for each diagnostic [7]. The flexible and scalable analysis framework features a parallel architecture that provides analysis workflow sequencing, data provisioning from various data sources, data mapping, and results archiving with pedigree (a record of the data inputs and analysis software version). Results are archived in NIF's data repository for experimentalist approval and display using a web-based tool.

SAVI tools provide experimental results within 30 minutes of an experiment through a web interface, with error bounds and quality metrics (see Figure 2-10). Because the data are stored in a relational content management system, the data can be viewed in various ways via the Data Visualization tool; within these web applications, experimentalists can choose to view the raw data or any level of the analysis. The system also tracks data metrics over time (trending) to detect systematic drifts that could affect data integrity. Comparisons to off-line data or simulations are also supported, and expected results are compared to measured data as part of the analysis. A key feature is that scientists can review data results remotely or locally, download results, and perform and upload their own analysis. SAVI was developed 


\section{National Ignition Campaign Program Completion Report}

in close collaboration with scientists who perform target experiments, to determine how the software tools could best support the scientists.

Experimental data as well as data on the post-shot state of the facility are housed in and retrieved (via the Archive Viewer) from the NIF data repository. This secure archive stores all the relevant experiment information - including target images, diagnostic data, and facility equipment inspections - for 30 years using a combination of high-performance databases and archival tapes. Retaining the data allows researchers to retroactively analyze and interpret results or perhaps to build on experimental data originally produced by other scientists. A crucial design feature of the database is that it preserves the pedigree of the data - all the linked pieces of information from a particular experiment, such as algorithms, equipment calibrations, configurations, images, and raw and processed data - and thus provides a long-term record of all the linked, versioned shot data.

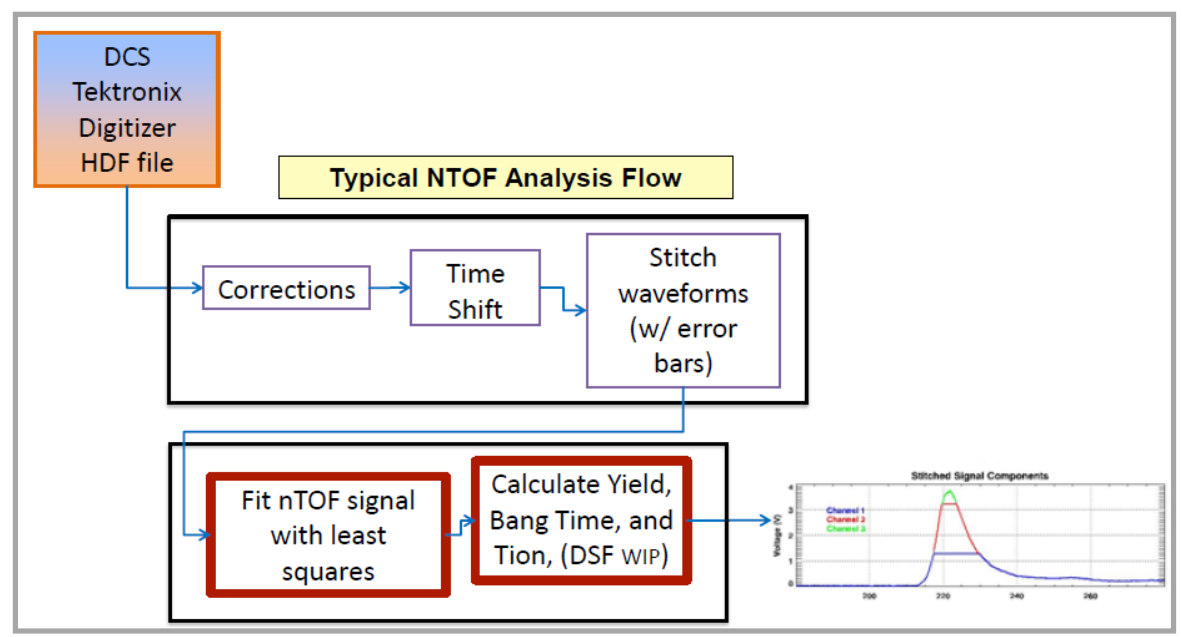

Figure 2-9. An example of diagnostic data flow for the Neutron Time-of-Flight detector. Accurate, precise, analyzed, and pedigreed data is available for visualization and download within minutes after the shot.

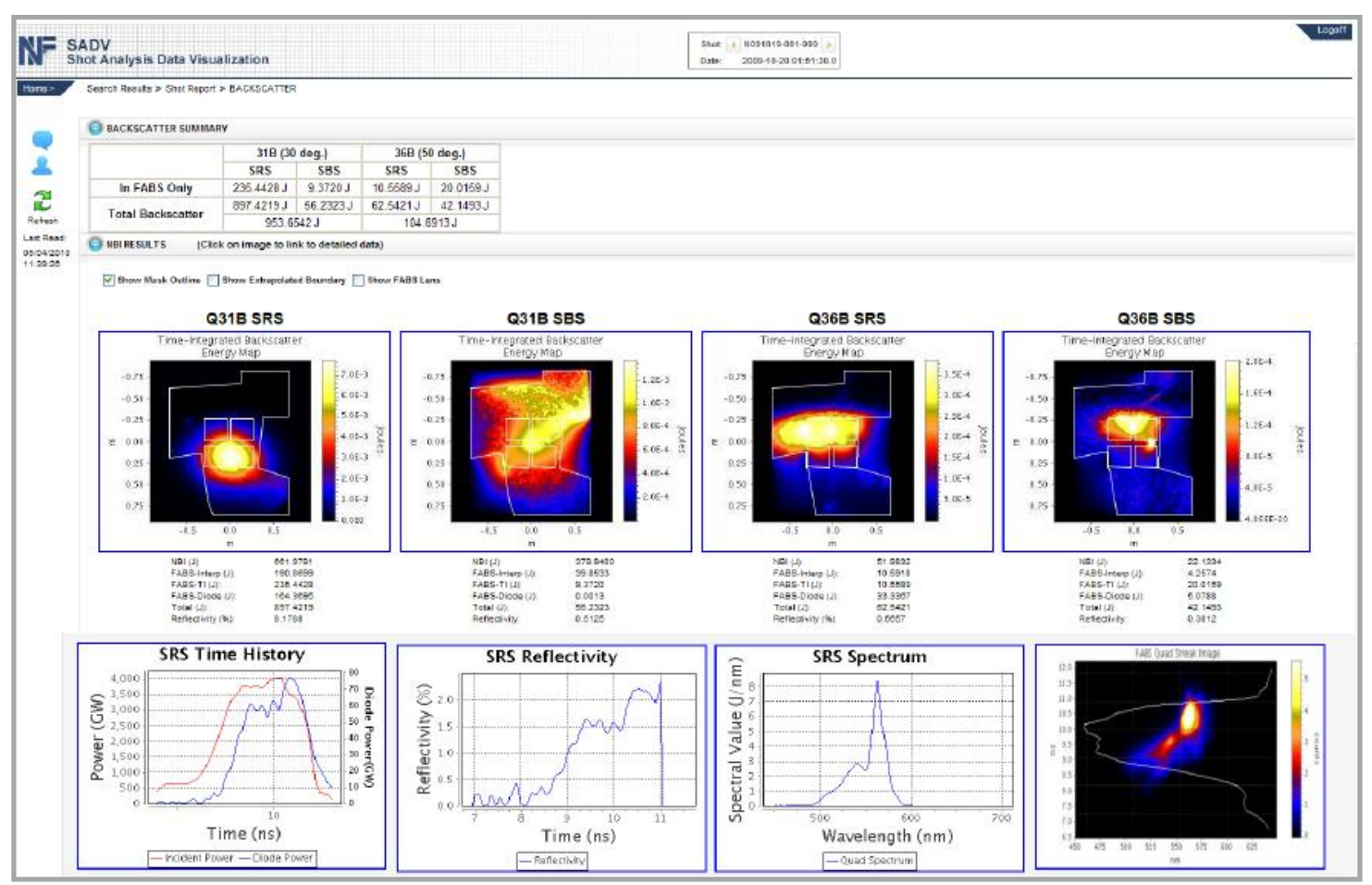

Figure 2-10. The shot analysis, visualization, and infrastructure tools help scientists quickly view and interpret data from a just-completed NIF experiment. 


\section{National Ignition Campaign Program Completion Report}

\section{NIC Wiki}

The NIC Wiki (https://nifit.llnl.gov/wiki/display/vc/Home) was created as a tool to improve collaboration and communication among the $100+$ scientists working across the ignition campaign. It provides a single, central location for quickly storing and accessing a diverse set of information and knowledge related to the campaign. The wiki stores user-generated content in a free-form format (presentations, documents, tables, charts, etc.). It is closely coupled, through numerous hyperlinks, to the NIF data repository- the official archival database for all NIF laser, target, and diagnostic data. This connection enables users to navigate quickly and easily between official shot data and scientific analyses and interpretation. Primary features of the NIC Wiki include the shot log, shot pages, campaign summaries and performance charts, meeting pages, and presentations (see Figures 12-11 through 12-15).

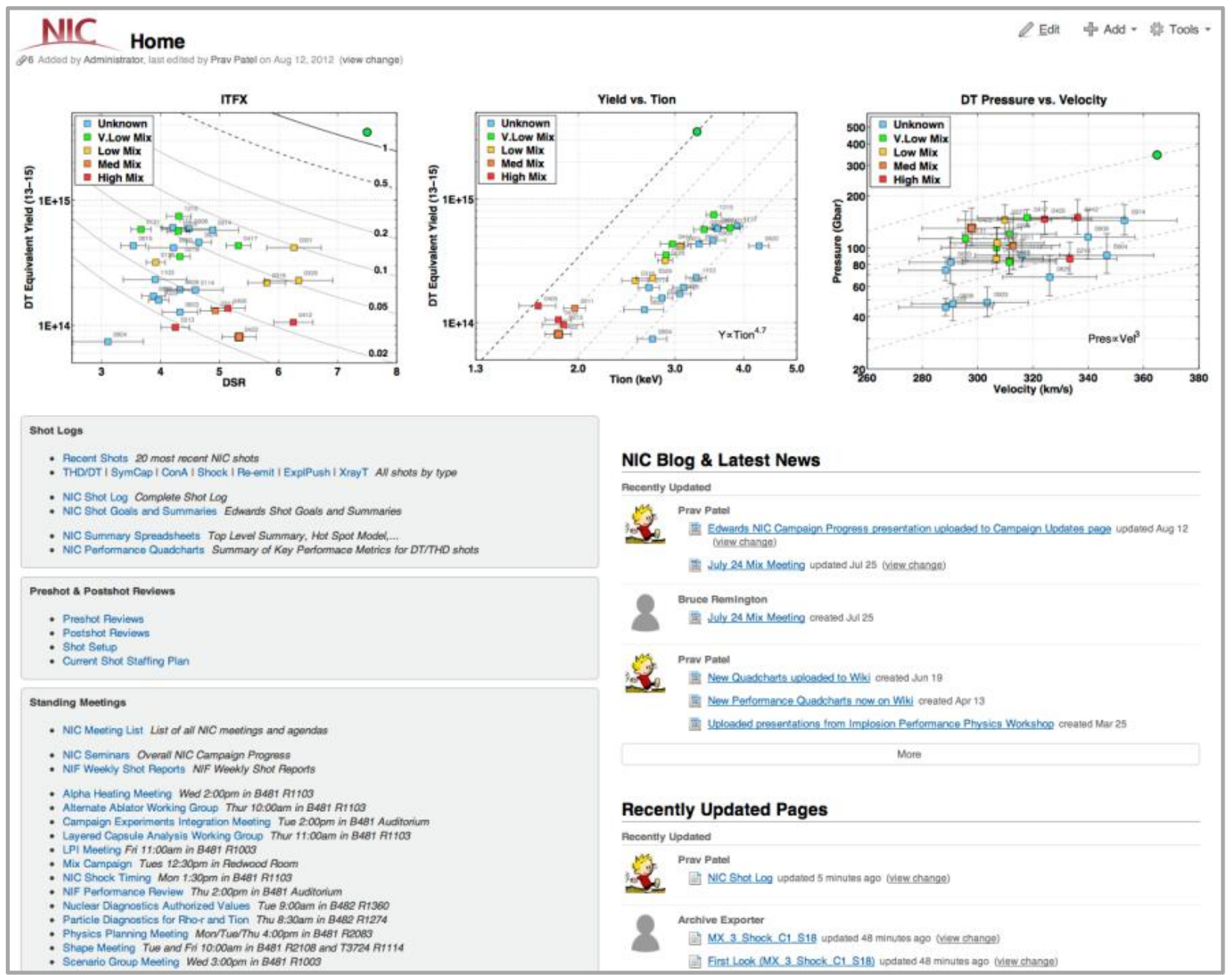

Figure 2-11. NIC Wiki home page displays latest shot results, links to wiki content, and latest news. 


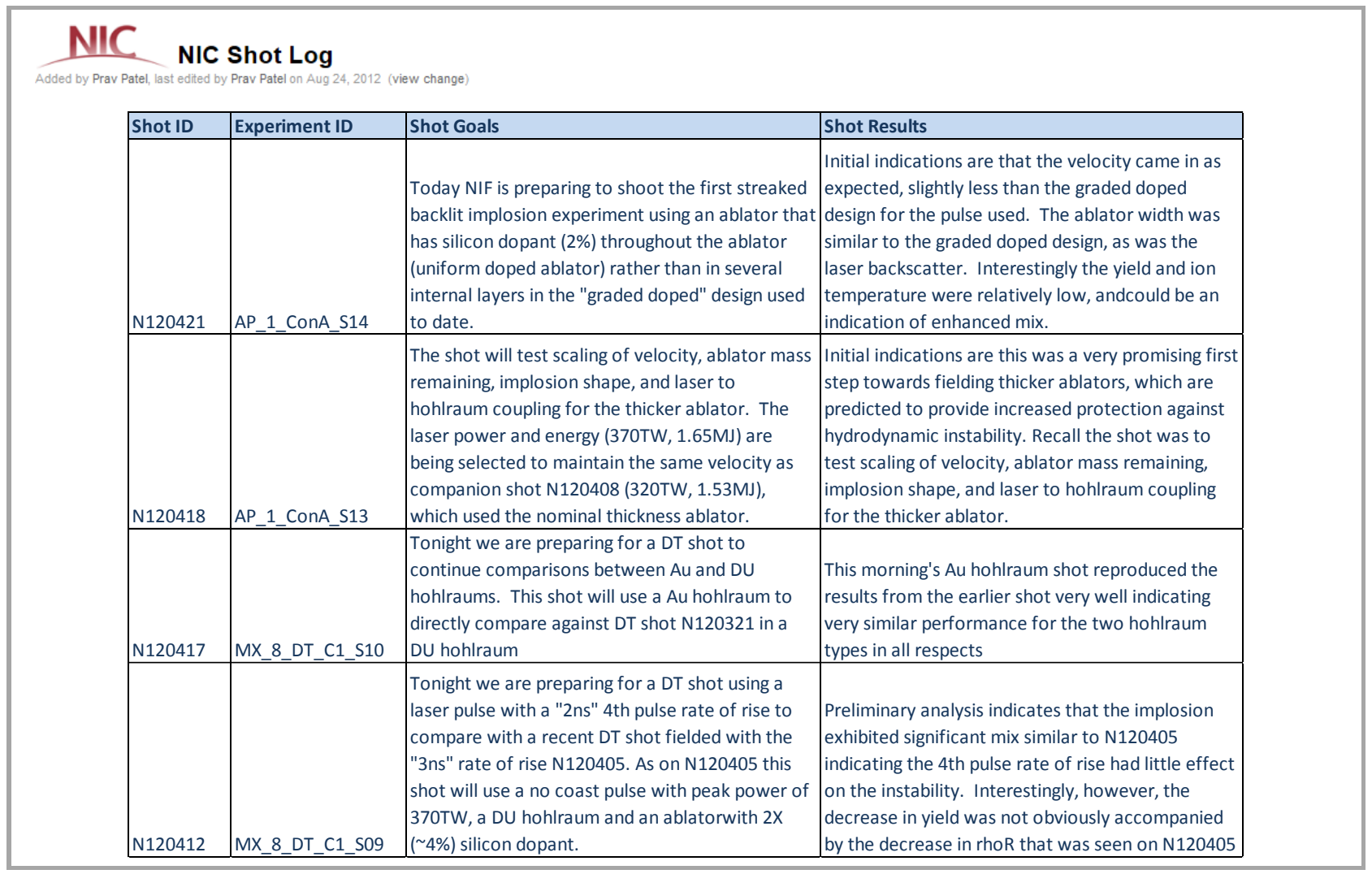

Figure 2-12. The shot $\log$ is a chronological record of all NIC experiments and includes the shot goals, shot results, and hyperlinks to the shot data in the data repository and to the wiki shot page.

\begin{tabular}{|c|c|c|c|c|c|}
\hline \multicolumn{6}{|c|}{$\begin{array}{l}\text { First Look } \\
\text { Campaign:MXIT_8_DT } \\
\text { Experiment:MXIT_8_DT_S02a }\end{array}$} \\
\hline \multicolumn{6}{|l|}{ Shot List } \\
\hline \multicolumn{6}{|l|}{ N120808-001-999 } \\
\hline Name & Size & Creator & Creation Date & Comment & \\
\hline 7] N120808-001-999_SPBT_Raw.pdf & $215 \mathrm{kB}$ & Dana Edgell & Aug 09, 2012 06:58 & & Properties | Remove | View \\
\hline Z7 N120808-001-999_SPBT_FTD1_analysis.... & $102 \mathrm{kB}$ & Dana Edgell & Aug 09, $201206: 58$ & & Properties | Remove | View \\
\hline SPBT_N120808-001-999.ppt & $188 \mathrm{kB}$ & Dana Edgell & Aug 09, $201206: 58$ & & Properties | Remove | Edit in Office | View \\
\hline N120808.pptx & $494 \mathrm{kB}$ & Riccardo Tommasini & Aug 09, $201211: 00$ & $\begin{array}{l}\text { Preliminary shape analysis } \\
\text { P } 0-0 \text {, Time Integrated }\end{array}$ & Properties | Remove | Edit in Office | View \\
\hline N120808-001-999 DT Quick look P90-7... & $1.08 \mathrm{MB}$ & Nobuhiko Izumi & Aug 15, 2012 19:55 & & Properties | Remove | Edit in Office | View \\
\hline FFLEX EHXI on N120808 rev2.pptx & $1.87 \mathrm{MB}$ & Tilo Doeppner & Aug 09, 2012 11:47 & EHXI analysis for $\mathrm{N} 120808$ & Properties | Remove | Edit in Office | View \\
\hline pTOF first look N120808-001.pptx & $83 \mathrm{kB}$ & Maria Gatu Johnson & Aug 09, 2012 13:10 & $\begin{array}{l}\text { Preliminary first-look pTOF } \\
\text { DT-n BT }\end{array}$ & Properties | Remove | Edit in Office | View \\
\hline N120808_GRH_Quicklookv1.pptx & $645 \mathrm{kB}$ & Jennifer Church & Aug 09, 2012 18:00 & & Properties | Remove | Edit in Office | View \\
\hline N120808_TC090-089_ARIANE_v02.pptx & $989 \mathrm{kB}$ & Vladimir Smalyuk & Aug 11, 2012 12:16 & $\begin{array}{l}\text { preliminary ARIANE shape } \\
\text { analysis }\end{array}$ & Properties | Remove | Edit in Office | View \\
\hline 國 N120808_ARIANE_frame_seq.avi & $517 \mathrm{kB}$ & Vladimir Smalyuk & Aug 11, 2012 12:16 & ARIANE movie & Properties | Remove \\
\hline N120808_TC90-078_HGXI_firstlook.ppt... & $743 \mathrm{kB}$ & Steven Glenn & Aug 15, $201211: 50$ & HGXI 90-78 preliminary & Properties | Remove | Edit in Office | View \\
\hline N120808-001-999_laser_produced_foot... & $9.85 \mathrm{MB}$ & Robert Kirkwood & Aug 09, 2012 15:07 & & Properties | Remove | Edit in Office | View \\
\hline 国 SXI_MXIT_8_DT_S02a_n10808-001-999.p... & $1.47 \mathrm{MB}$ & Marilyn Schneider & Aug 10, 2012 14:48 & Standard SXI report & Properties | Remove | Edit in Office | View \\
\hline 國 n120808_NI_quicklook_v1.pptx & $1.55 \mathrm{MB}$ & Gary Grim & Aug 09, 2012 09:30 & & Properties | Remove | Edit in Office | View \\
\hline 直 N120808_00_00_frame_seq.avi & 844 kB & Arthur Pak & Aug 14, 2012 20:42 & $\begin{array}{l}\text { HGXI 00-00 frame } \\
\text { sequence. }\end{array}$ & Properties | Remove \\
\hline (120808_TC000-000_HGXI_V01.pptx & $1.39 \mathrm{MB}$ & Arthur Pak & Aug 14, 2012 20:41 & HGXI 00-00 first look slides & Properties | Remove | Edit in Office | View \\
\hline 国 N120808_TC090-078_frame_seq_green.a... & $795 \mathrm{kB}$ & Steven Glenn & Aug 15, 2012 11:29 & HGXI 90-78 movie (green) & Properties | Remove \\
\hline [- N120808_TC090-078_frame_seq.avi & $920 \mathrm{kB}$ & Steven Glenn & Aug 15, 2012 11:29 & HGXI 90-78 movie & Properties | Remove \\
\hline
\end{tabular}

Figure 2-13. A unique shot page for each shot is used to store and share results and data analysis for the shot, along with pre-shot and post-shot reviews. 


\section{National Ignition Campaign Program Completion Report}

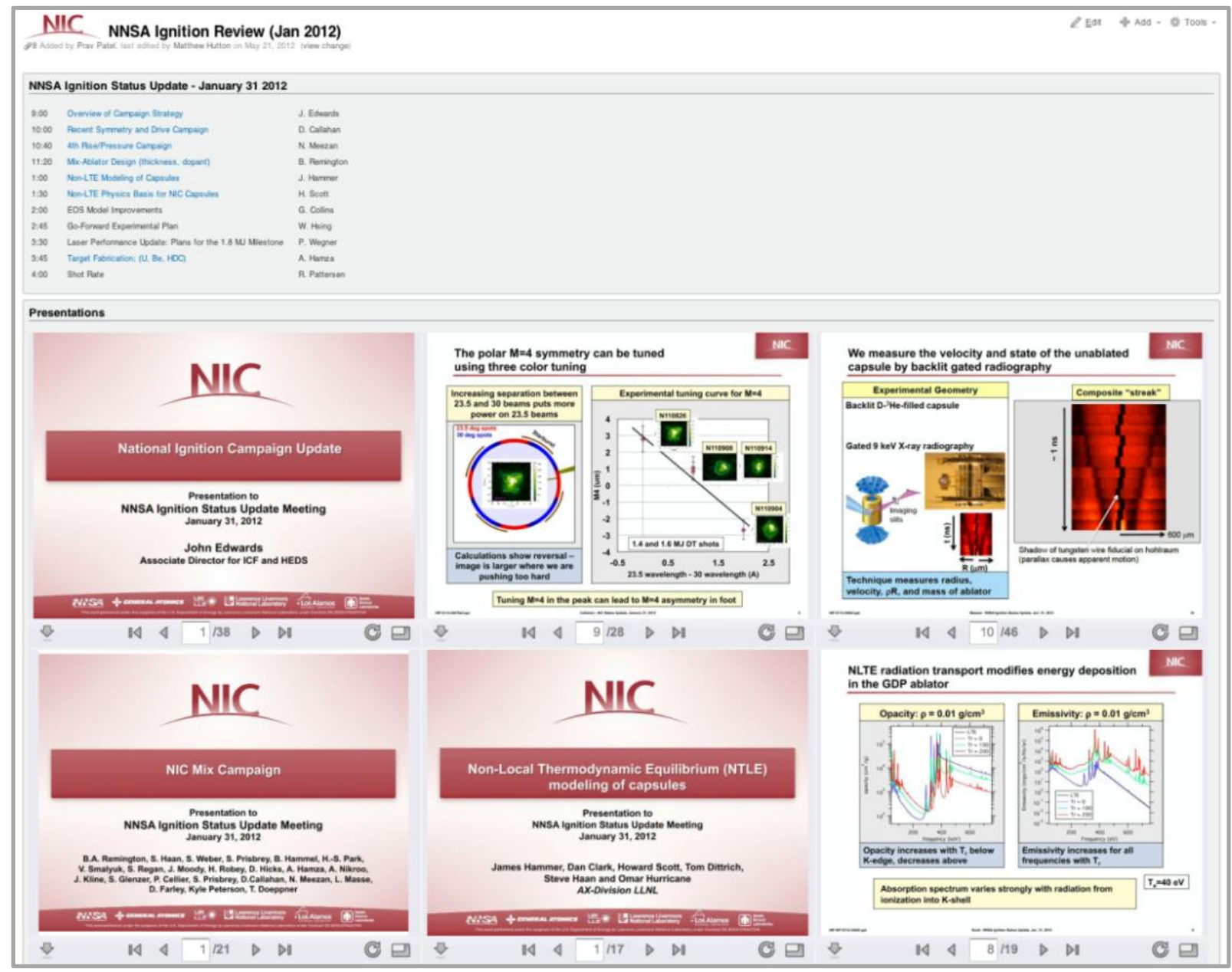

Figure 2-14. All scientific standing meetings maintain wiki pages to record discussions and presentations and track agendas and action items. The wiki is also used to archive presentations from numerous internal workshops, internal and external reviews, and external conferences. 


\section{National Ignition Campaign Program Completion Report}

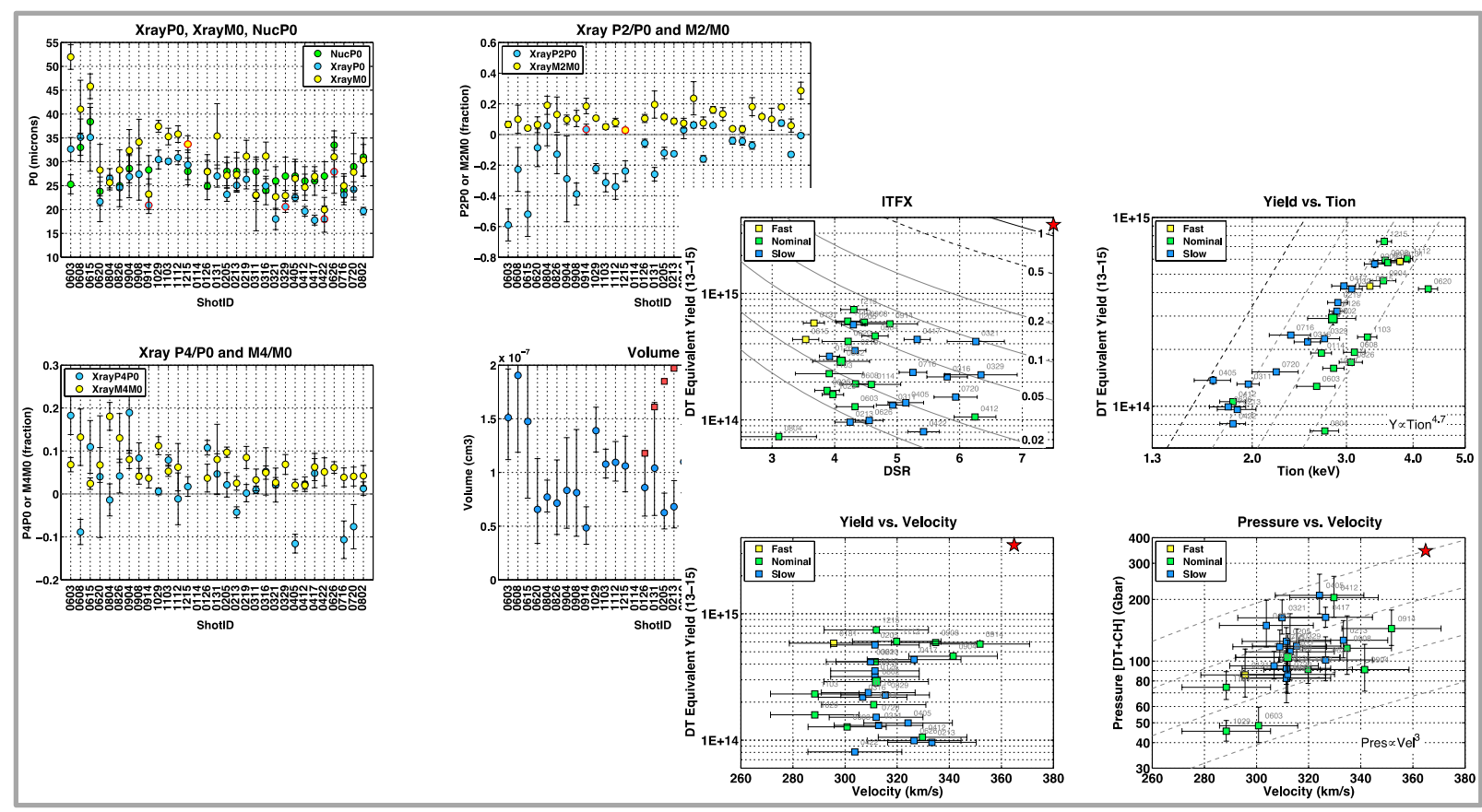

Figure 2-15. The Campaign Summaries and Performance Charts contain top-level summaries of NIC experimental data and simulation results. Key performance metrics of the implosions are tracked across shots and in scores of auto-generated charts with filters to separate out selected laser or target characteristics (dopant level, fourth pulse rise, hohlraum type, etc.) This compilation of data and simulation has assisted in assessing the overall campaign progress and exploring trends and correlations.

\section{Additional Systems Engineering Capabilities}

NIF's Systems Engineering organization has been developing and providing to users other essential support and organizational capabilities for successfully conducting experimental campaigns on NIF. These include expert group review and analysis, requirements management, experiment control, system integration, configuration management, and a Work Authorization Point (WAP) process.

\section{Expert Group Review and Analysis}

While LPOM ensures that the requested laser pulse can be generated safely and effectively, expert groups provide an additional level of shot analysis and oversight. NIF's System Engineering organization has a number of expert groups that are consulted throughout the shot preparation and planning process. These expert groups also formally review experiment plans at the Implementation Review.

The NIF expert groups are as follows:

- Beamline and Laser Integrated Performance (BLIP): BLIP works with the Responsible Individual and/or Principal Investigator to set final laser and user optic specifications for each experiment. This group coordinates with the NIF Optics Loop (NOL) group as needed.

- Target and Laser Interaction Sphere (TaLIS): The TaLIS group provides expert group review, evaluation, and recommendations on issues in the NIF target chamber, including experimental campaign planning and shot setup reviews and online commissioning activities.

- NIF Optics Lifetime: The optics expert group provides an assessment of NIF campaign cost and feasibility with regards to final optics use and the required capacity for supporting loop infrastructure.

- Cleanliness and Materials: This group ensures that NIF cleanliness protocols are maintained in the target chamber, Diagnostic Instrument Manipulators (DIMs), and other areas. 


\section{National Ignition Campaign Program Completion Report}

- Beryllium/Uranium/Tritium/Yield (BUTrY): BUTrY provides guidance or resolution to directorate managers and authorizing individuals on environmental safety and health issues related to the use of beryllium and radiological safety.

\section{Requirements Management/Experimental Control}

User requests to change the pulse shape, energy, power, or pulse length are evaluated by NIF Systems Engineering to determine whether the laser system can operate safely and effectively with the requested parameter in place. Requirements are reviewed by the BLIP expert group as communicated through the Campaign Management Tool. Performance calculation using LPOM/VBL and expert group review may result in modifications to experiment setup to meet requirements and also may motivate definition of preexperiment calibration activities or performance tests.

\section{Configuration and Requirements Management}

Standard configuration and requirements processes are used to ensure that work is performed in a manner that is consistent with safety and security. In particular, configuration management is the process by which any desired changes to the authorized policies, procedures, operations, and equipment are allowed only after a formal review process involving all stakeholders. The configuration management process is applied in a risk-based graded manner, and change requests are managed through the use of a change control board.

For more information on NIF configuration management, see I.8, Operational Capabilities, and the NIF and Photon Science Configuration Management Plan [8].

\section{Work Authorization}

Shot planning and execution are managed using the WAP process. This rigorous authorization process confirms that all aspects of an activity - including equipment, personnel, and procedures - have been prepared safely, securely, and efficiently.

\section{References}

1. M. Shaw et al., "Laser Performance Operations Model," Opt. Eng., 43, 2885-2895 (2004).

2. M. Shaw et al., "Performance and Operational Modeling for the National Ignition Facility," at International Symposium on Optical Science and Technology (2003)

3. M. Shaw et al., "Laser Performance Operations Model (LPOM): A computational system that automates the setup and performance analysis of the National Ignition Facility," presented and the $5^{\text {th }}$ International Conference on Inertial Fusin Sciences and Applications, J. of Physics: Conference Series 112032022 (2008).

4. J. T. Hunt, K. R. Manes, J. R. Murray et al., "Laser design basis for the National Ignition Facility," Fusion Technology 26 767-771 (1994).

5. C.A. Haynam, R.A. Sacks, and M.J. Shaw, "Computational modeling in support of the National Ignition Facility," International Conference on Accelerator and Large Experimental Physics and Controls Systems (ICALEPS) (2001).

6. L.M. Frantz and J.S. Nodvik, "Theory of pulse propagation in a laser amplifier," J. Appl. Phys. 34, 2346 (1963).

7. S. Azevedo et al., "Automated Experimental Data Analysis at the National Ignition Facility," presented at International Conference on Accelerators and Large Experimental Physics Control Systems, Kobe, Japan, October 13, 2009.

8. NIF and Photon Science Configuration Management Plan, NIF-5018949. 


\section{National Ignition Campaign Program Completion Report}

\section{I.3-TARGET PHYSICS}

The main scope of work conducted under the NIC WBS Element I.3, Target Physics, is developing theories and models, and performing calculations to validate the ignition point design; and designing experiments on NIF and supporting facilities to verify aspects of the design. In addition, target design activities on alternative designs for risk mitigation may be performed. The experimental component of this element designs and executes ignition experimental campaigns as well as supporting experiments on NIF and other facilities for verification, validation, and risk mitigation. This area also supports developing requirements for diagnostics, targets, and cryogenic layering in support of ignition experimental campaigns. Target Physics may participate in the development and implementation of specialized equipment, as required, for ignition and HED experimental campaigns on NIF and for supporting facilities to execute the ignition plan.

The goal of the indirect-drive ignition program is to achieve routine indirect-drive ignition on the NIF by the end of FY2012. The goal of the direct-drive physics effort is to support the indirect-drive ignition program. Polar Drive (PD) may also result in an alternative approach to ignition and, subject to a separate review and approval process of a PD project execution plan proposal, a PD project may ultimately be fielded on the NIF. See Section $\mathrm{f}$ for a description of the progress in direct-drive ignition during the NIC and its impact on the indirect-drive ignition campaign.

\section{A. Indirect Drive Overview}

Progress on NIF capabilities and the NIC experimental campaign since the start of precision optimization experiments in May 2011 has been considerable and can be summarized as follows:

- Diagnostics, targets, and laser capabilities have reached the levels of performance needed for a systematic optimization of ignition-scale experiments.

- Hohlraum temperatures have been achieved that exceed the $300 \mathrm{eV}$ point design goal with nearly constant laser energy coupling of $84 \pm 2 \%$ for energies from 1.2-1.8 MJ.

- Hot spot symmetry that meets ignition specifications has been achieved using a combination of power balance and wavelength shifts between the inner and outer beams, and an additional wavelength shift between the two cones of inner beams.

- The dependence of implosion velocity on ablated mass has been accurately measured and is consistent with code simulations within the error bars. These measurements have enabled the identification of a mix performance boundary that depends on the velocity and the remaining unablated mass - a focus of the go-forward experimental plan.

- The NIC experiments have demonstrated an increase in the fuel areal density ( $\rho r$ ) from $\sim 35 \%$ to $\sim 85 \%$ of that specified for the point design by implementing systematic improvements to the shock timing, hot spot symmetry, and laser pulse shape.

- The stagnation pressure of the hot spot is $\sim 40 \%$ of point design goals.

- The ITFX of recent experiments has reached $\sim 0.1$. The improved performance was achieved at a lower implosion velocity and lower laser power than the previous best performing capsule, providing a greater margin for the path to ignition.

- For the first time in the laboratory, the beginning of alpha heating has been inferred. In the best implosions thus far, about $\sim 0.5 \mathrm{~kJ}$ of alpha particle energy is produced in the hot spot, the areal density of which is sufficient to trap a significant fraction of this energy. In these implosions, the total nuclear yield was $\sim 2.5 \mathrm{~kJ}$ which is also comparable to the thermal energy in the hot spot.

- As the NIC has moved toward shells with optimized shock timing and extended drives to achieve higher pressure and higher fuel $\rho$ r, the effect of mix on implosion performance has been observed and quantified, and is being investigated.

A key element in the progress toward ignition has been integration into the experimental platforms of a wide range of improvements in the technological capabilities of the NIF laser, the targets, and the diagnostics, including: 


\section{National Ignition Campaign Program Completion Report}

- Steady advances in laser performance enabled the longer pulses requiring increased laser energy used in recent experiments. During the March and April 2012 experiments, the NIF laser routinely delivered pulse energies of 1.45-1.8 MJ with powers over $400 \mathrm{TW}$. In July 2012, NIF delivered an ignition pulse with the full NIF design energy and power of $1.8 \mathrm{MJ}$ and $500 \mathrm{TW}$.

- A steady increase in the number of diagnostics and their performance. Approximately 60 diagnostic systems are now in use.

- Significant advances in the characterization and precision of targets used for these experiments.

With the current fuel areal density, ${ }^{\mathrm{p}}$ if yields of the experiments are increased by a factor of about 5 , the threshold of alpha-dominated burn would be reached. This will require about a $2 \mathrm{x}$ increase in the central hot spot density at the ion temperatures currently achieved. There will be three principal elements of the experiments designed to broaden the range of data available:

- Implosions with reduced sensitivity to hydrodynamic instability.

- Enhanced radiography of the implosion

- New platforms for improved understanding of target performance.

\section{B. Indirect-Drive Approach}

To set the point design requirements, the NIC team used numerical algorithms and databases integrated into models that have been extensively tested on more than three decades of experiments on the Nova and Omega lasers and their predecessors. These requirements were used to establish system capabilities for the laser, targets, diagnostics, and facility infrastructure. Using these system capabilities, a sequence of experimental platforms were designed to measure and tune a variety of physical parameters needed to optimize the implosion of the fuel. Finally, these optimized target and laser parameters were applied to cryogenic layered implosions whose performance was assessed using a variety of diagnostics that measured the implosion outputs.

NIC used indirect drive, also called x-ray drive. In these targets, the 192 high-energy NIF [1,2] laser beams enter a high-z cylinder, the hohlraum, through laser entrance holes (LEHs) on the ends of the cylinder. The plastic (CH) point design target used in NIC experiments in 2010-2012 is shown in Figure 3-1.

${ }^{\mathrm{p}}$ Areal density (abbreviated $\rho \mathrm{r}$ ) is a measure of the combined thickness and density of the fuel shell in an ignition target. It is a function of how much energy is absorbed by the fuel and the accuracy of target conditions during implosion. 


\section{National Ignition Campaign Program Completion Report}

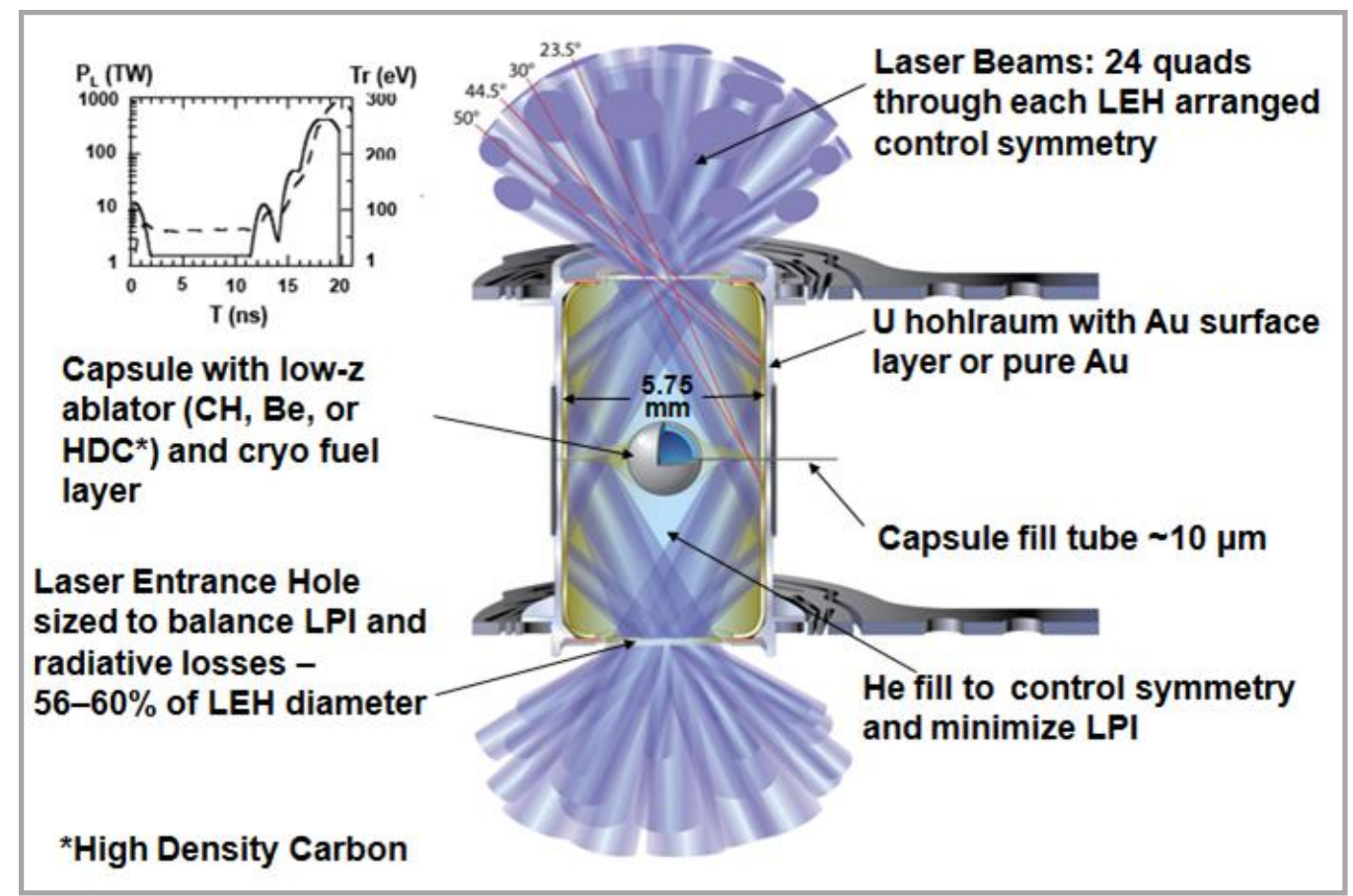

Figure 3-1. Ignition target design. For the initial ignition campaign using a $\mathrm{CH}$ capsule, the hohlraum diameter was $5.44 \mathrm{~mm}$; it was later increased to $5.75 \mathrm{~mm}$. Initially, the hohlraum wall material was all Au. A uranium hohlraum reduces the peak power requirements by $\sim 30 \mathrm{TW}$ and has been used in recent experiments. Beryllium and HDC (nanocrystalline diamond) ablators will be tested in later campaigns. Also indicated is the laser pulse shape showing the laser power in terawatts and the radiation temperature reached at that power vs. time in nanoseconds.

The $\mathrm{X}$-rays that result from heating the high- $\mathrm{Z}$ walls of the hohlraum ablate material from the spherical shell surrounding the fuel, which is mounted in the center of the hohlraum. The resulting implosion compresses and heats the central fuel to fusion conditions. The central capsule containing the fuel is made up of thin concentric spherical shells. The outer shell can be $\mathrm{CH}$ plastic, beryllium, high-density carbon (nanocrystalline diamond), or another low- $\mathrm{Z}$ material. The layers of the shell must be very smooth, to minimize seeds of hydrodynamic instabilities. Figure 3-2 shows the initial $\mathrm{CH}$ point design with silicon

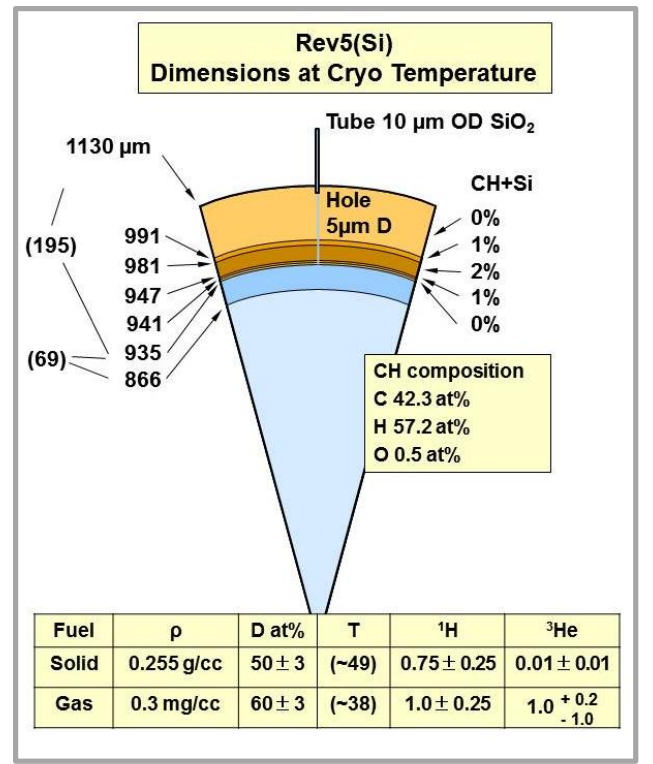

Figure 3-2. Point design capsule with silicon dopant preheat shielding. The silicon dopant profile and shell thickness were modified throughout the NIC to optimize the implosion, as discussed below.

The key challenges for NIC were:

1. Designing a laser/target system that can achieve fusion ignition and burn propagation;

2. Achieving the conditions required for ignition in the presence of uncertainties in the physics models; and

3. Gauging progress toward achieving those conditions as the ignition campaign progressed.

Defining these challenges, developing the approaches to address them, and providing the capabilities to achieve the subsequent requirements on the laser, target fabrication, and experiments has been the focus of research and experimentation in ICF for more than four decades.

A key advancement to the ICF strategy that occurred during NIC was development of a method of reducing the effective 


\section{National Ignition Campaign Program Completion Report}

dimensionality of the ignition campaign. This is shown schematically in Figure 3-3. There are hundreds of actionable quantities that affect an ICF implosion. These can be binned by the physical instantiation of the laser and target configuration along with specifications and allowable variations in experiments. These quantities can also be categorized as either one-dimensional variables (e.g., capsule dimensions and dopants, laser power and shock timing precision) or three-dimensional variables (e.g., ablator/fuel roughness, pointing errors and beam-to-beam power balance).

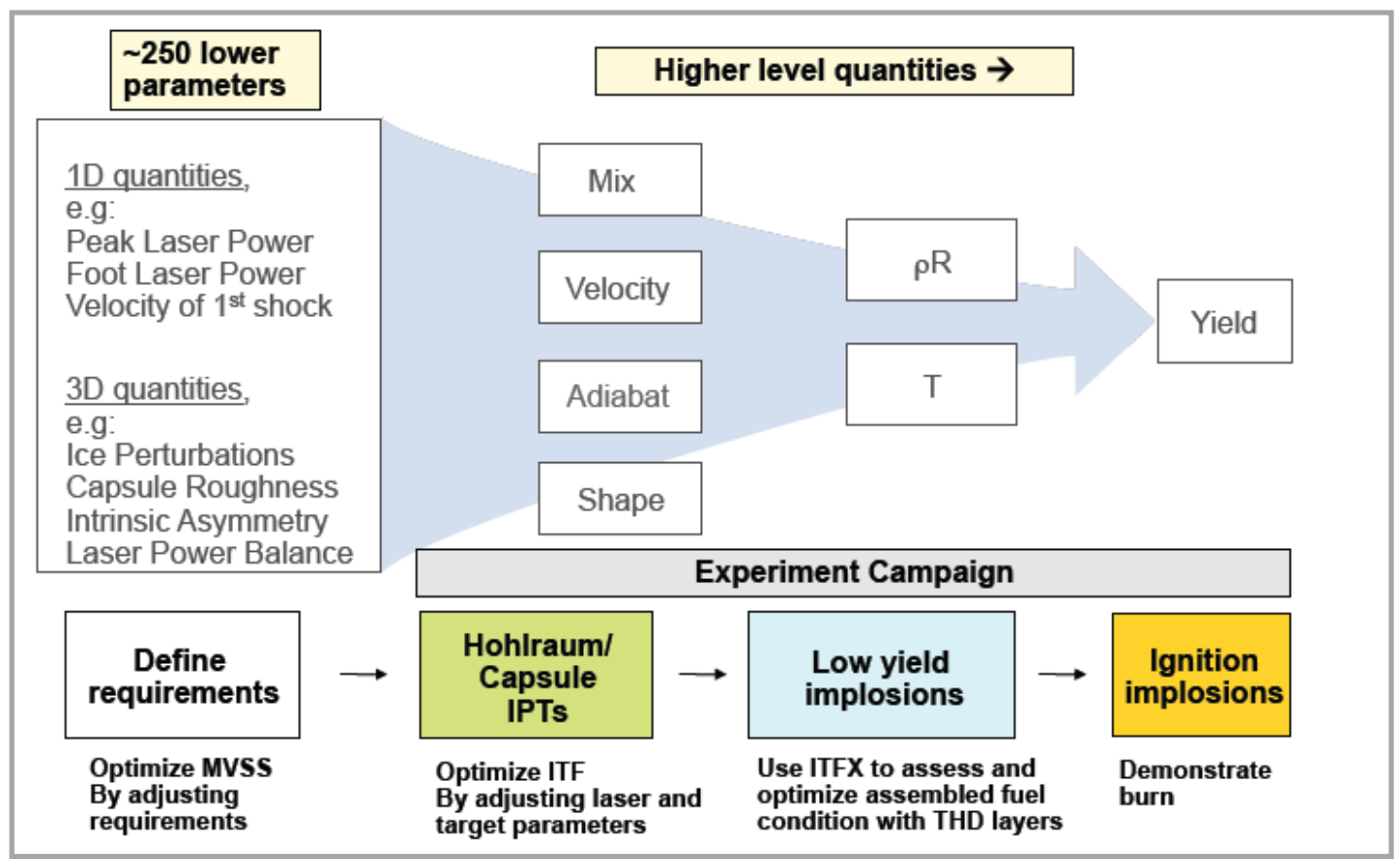

Figure 3-3. The NIC campaign methodology is designed to reduce the dimensionality of the experimental campaign. The NIC team identified four primary input variables (velocity, adiabat, hot spot shape, and fuel/ablator mix) and an ignition threshold factor based on those variables. Together, these variables determine the final state of the fuel. In cryogenic layered non-ignition implosions, the total fuel pr, the hot spot $\rho r$, temperature, and an output parameter ITFX based on those quantities are identified to assess the quality of the assembled fuel.

The NIC team showed that these variables can be mapped onto the principal in-flight parameters of the implosion process to address Challenge 1 , above, by optimizing the implosion velocity (V), the fuel entropy or adiabat $(\alpha)$, the hot spot shape $(\mathrm{S})$, and the mix of ablator into the fuel $(\mathrm{M})$. A metric termed the Ignition Threshold Factor (ITF) [3-8] was developed for setting specifications on the laser, targets, and experiments and for assessing the quality of the results achieved in experiments relative to those required for ignition. The target platforms developed to achieve the specifications for these key input parameters and the experimental measurements on these platforms [9] address Challenge 2, above. The principal outputs required to address Challenge 3, above, have been reduced to a measurement of the neutron yield (Y) and the fraction of those neutrons down scattered (DSR) by the surrounding cold fuel in an implosion with a cryogenic fuel layer. The yield is a measure of the hot spot performance, and the DSR is a measure of the total pr of the fuel, which determines confinement time. A metric [10] has been developed called ITFX, which is an Experimental Ignition Threshold Factor based on the yield and DSR. Both the ITF and ITFX metrics are equivalent to a Generalized Lawson Criterion [11,12] for ICF.

The basic requirements for ignition of DT fuel in an inertially confined implosion have been known for decades [13]: a central hot spot of 4-5 keV with a size comparable to the range of an alpha particle $\sim 0.3$ $\mathrm{gm} / \mathrm{cm}^{2}$, surrounded by a confining layer with a $\rho \mathrm{r}>1 \mathrm{~g} / \mathrm{cm}^{2}$ to provide adequate confinement. These are the conditions for the compressed state of the fuel, the endpoint or outputs of a properly tuned implosion. These conditions are similar to the $\mathrm{n} \tau$ and $\mathrm{T}$ specified by the Lawson Criterion in magnetically confined fusion plasmas. Under these conditions, the alpha heating can generate a self-sustaining burn wave, with 


\section{National Ignition Campaign Program Completion Report}

no external energy input, which heats the fuel surrounding the hot spot to tens of keV. This burn wave is well established by the time yields reach an energy equal to the input laser energy, and this level of performance, as recommended by the 1997 NRC review of NIF [14], has been adopted as a working definition of ignition. At this yield, the thermonuclear energy produced as a result of alpha heating is about 100 times the peak kinetic energy imparted to the fuel during the implosion. Shown in Figure 3-4 is a plot for the NIC point design of the fraction of the yield observed that is the result of heating from alpha particle deposition.

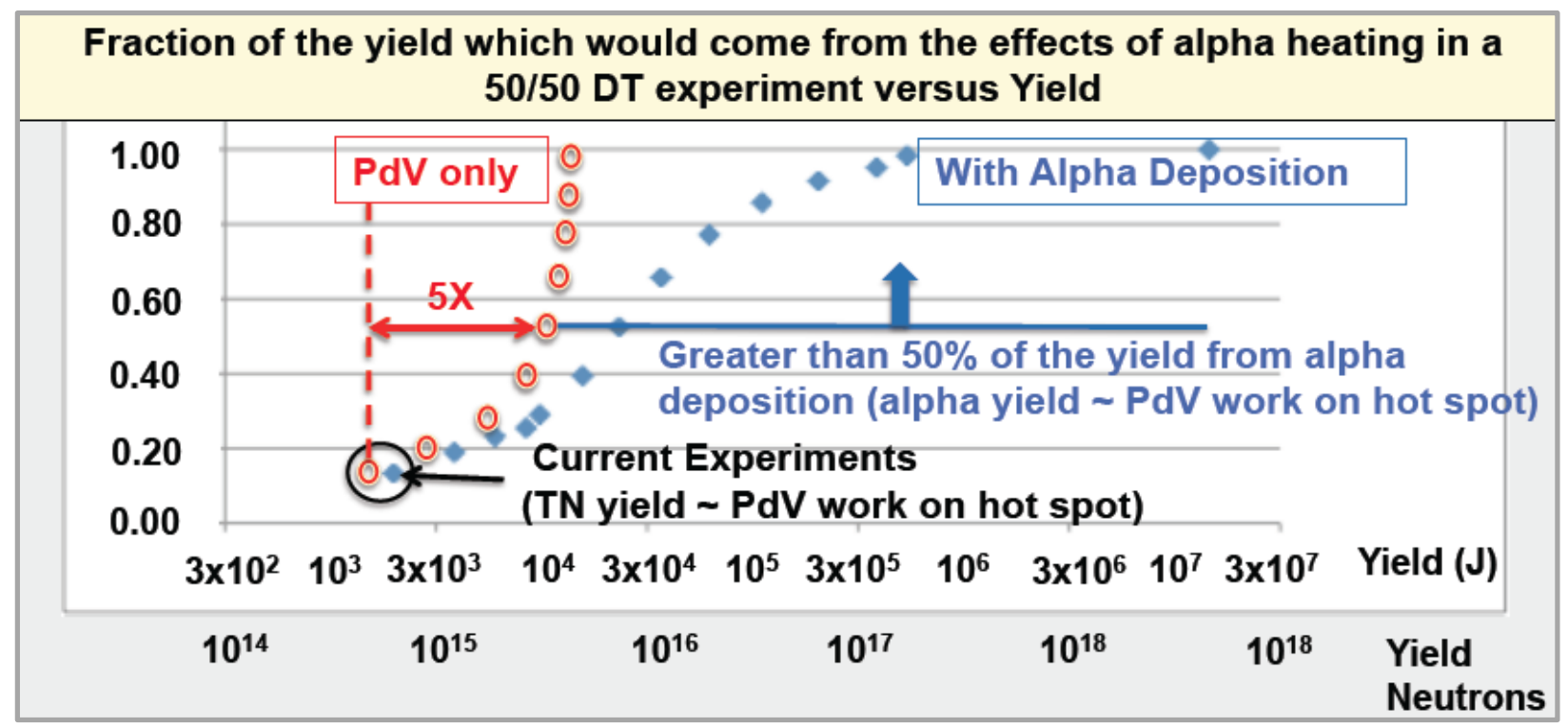

Figure 3-4. Impact of alpha heating on the yield. Above a yield from PdV work of about 8-10 kJ, the fusion burn becomes alpha dominated, with more than half the yield from alpha deposition.

There is a continuum of effects from alpha heating as the yield and fuel $\rho$ r increase toward ignition and beyond to high gain. For the first time in laboratory ICF, the hot spot $\rho$ r in the NIF implosions is sufficient to stop the alpha particles being produced by the thermonuclear reactions occurring within the hot spot. With the compressions currently being achieved in the NIC implosions, as described below, the pr of the hot spot is about $0.1 \mathrm{~g} / \mathrm{cm}^{2}$, which is sufficient to stop the alpha particles at the $\sim 3 \mathrm{keV}$ temperature of the hot spot. With total thermonuclear yields of only a couple of kilojoules, about $10-15 \%$ of the yield would come as a result of alpha heating, as shown in Figure 3-4. A yield of $2 \mathrm{~kJ}$ is about equal to the compressive work done on the central hot spot. This is about $20 \%$ of the total fuel kinetic energy at peak implosion velocity. The alpha particle energy is $20 \%$ of the TN yield or $400 \mathrm{~J}$ from $2 \mathrm{~kJ}$ of yield. Calculations indicate that a yield from PdV work of 7-10 kJ, or $1.4-2 \mathrm{~kJ}$ of alpha particles, is sufficient to produce an ion temperature increase of about $0.5 \mathrm{keV}$ and to double the yield as indicated in Figure 3-4. Beyond this point, the thermonuclear burn is alpha dominated. At a yield of $1 \mathrm{MJ}$, about $99 \%$ of the yield is the result of heating from alpha deposition, and burn propagation from the initial hot spot is well underway.

\section{Use of Numerical Models within the NIC}

Development of the foundational science of ICF requires the integration of an exceptionally wide range of physical phenomena. ICF computational models incorporate this accumulated knowledge into a system of equations, algorithms, and databases, with calculations carried out on state-of-the-art computers [15] where the capabilities have increased more than three orders of magnitude in the past decade. The two principal codes used for radiation hydrodynamics calculations on the NIF are Lasnex [16] and HYDRA [17]. The principal codes used for modeling laser-plasma interaction (LPI) physics is the pF3D [18] code. The models were developed and tested using a wide range of experiments on the Nova and Omega lasers and other facilities. However, these experiments were carried out with nearly a factor of 100 less energy 


\section{National Ignition Campaign Program Completion Report}

than NIF. Accordingly, these models were not tested over the full range of spatial and temporal scales or temperature and density conditions required for ignition experiments on the NIF. Because of the complexity of the scientific issues involved, and the difficulty of solving the equations describing these phenomena, even with today's computers these computational models necessarily involve approximate solutions of the relevant physics. While recognizing that there will inevitably be areas where the models prove inaccurate, a key challenge in the pursuit of ignition is to identify ways to use the models, together with experimental data from the campaign in an iterative manner, to optimize progress toward ignition.

Although the models were not expected to be sufficiently accurate to allow a priori specifications of precise details of the targets and laser pulse, the extensive data base acquired on all the principal ICF physics phenomena provided confidence that the specifications set by the codes could provide a good starting point for the campaign. The campaign would then be iterated using new experimental data. Prior to the start of experiments, the codes were used to set the initial laser pulse and target specifications, as well as experimental inputs, including expected timing and signal levels for the various diagnostics. "Playbooks" of sensitivities to adjustments in the experimental inputs were generated and used to optimize implosion performance. The expectation was that in some cases, the models would prove adequate to specify experiments that would achieve the required performance in relatively few iterations. In other cases, the models were expected to disagree with the data to a sufficient extent that additional experiments would be necessary to probe more deeply into the underlying physics. This approach has allowed the NIC team to move quickly through those areas of the physics where the models proved to be adequate while focusing resources and attention in the areas where the data was at greater odds with the models. This approach helped identify those areas of the underlying physics where improvements would have the largest impact, both in current experiments and for future applications where a more a priori predictive capability would allow more rapid convergence to the required performance. In advance of experiments, it was not possible to know which areas of the physics would prove to be the most challenging.

As data were obtained, they are fed into the models. On a time scale of days to months, the models were adjusted to obtain better correspondence between the calculations and the observations. A number of factors can contribute to the differences between experiment and calculation, but this kind of adjustment of the models has been shown to be quite effective as a means for predicting changes to upcoming experiments that are necessary to move the results closer to those required for ignition. Once enough experimental data has been obtained, the measured sensitivities themselves can be used for further adjustments. This process has been used to optimize symmetry, adiabat, and implosion velocity; examples are given below. On a longer time scale, which can range from months to a year or more, the underlying physics equations, algorithms, or data bases are improved as necessary to obtain a better a priori predictive capability. Areas in which the models need further improvements are also given below.

\section{Indirect Drive Implosion Optimization}

Many lower-level variables affect the four principal quantities in ITF. However, extensive analysis of the ignition point design indicated that, given a target and laser that meet specifications, a relatively small number of high-level variables have the largest leverage in the process of optimizing the $\mathrm{V}, \alpha, \mathrm{S}$, and $\mathrm{M}$ of an implosion. Based on an assessment of the variables optimized in computer simulations to achieve ignition and propagating burn, an initial set of laser and target parameters was identified that had be set precisely in pre-ignition experiments to optimize the imploded fuel assembly as required for ignition. However, it was expected that experiments would uncover the necessity for a more extensive optimization of features such as the pulse shape, capsule structure, and hohlraum geometry.

Optimization of these high-level variables formed the basis for an array of basic target platforms used in the implosion optimization campaign, as well as for the measurement precision required by NIF as discussed extensively in the article by Landen et al. [9] The principal target platforms are Symmetry Capsules (Symcaps), Re-emits, Keyholes, and Convergent Ablators (ConA), described below. NIC involved a series of iterations on implosion optimization in which the non-layered surrogate targets are 


\section{National Ignition Campaign Program Completion Report}

interleaved with layered THD and DT targets. In this way, it can be determined if the expected improvement in performance is achieved as the precision of the implosion inputs improves. Since the beginning of precision optimization implosion experiments in May 2011, a wide range of experiments addressing each of the key implosion attributes has been carried out.

Specifications for the accuracy with which the key variables must be established in tuning campaign surrogate targets are set by a multivariable sensitivity study (MVSS) designed to achieve a global optimization of specifications for the laser, targets, and experiments. The MVSS carried out for the ignition point design is discussed in an article by Haan et al. [3] These requirements, along with the experimental observables and their required measured precision, are discussed in a paper by Landen et al. [9] The requirements on these measurements set the goals of the precision optimization, or "tuning," campaign. Tuning requirements for each of the implosion optimization platforms are defined so that implosions meeting these requirements would meet the ITF specifications set for the point design. A summary of those requirements and the precisions (shot-to-shot uncertainty in measurement) and accuracies (absolute error in measurement) that have been achieved is given in Table 3-1. Key results from each of the implosion optimization platforms are described below.

Table 3-1. Required and achieved precision and accuracies for each of the implosion optimization platforms. Color coding: met or exceeded requirement (blue), on track to meet requirement (green), not applicable (yellow).

\begin{tabular}{|c|c|c|c|c|c|}
\hline \multirow{2}{*}{ Platform } & \multirow{2}{*}{ Key Parameter } & \multicolumn{2}{|c|}{ Precision } & \multicolumn{2}{|c|}{ Accuracy or Goal } \\
\hline & & Budget & June 2012 & Spec & June 2012 \\
\hline Reemit & Picket Symmetry (P2) & $\pm 3 \%$ & $\pm 1 \%$ & $\pm 7.5 \%$ & $\pm 2 \%$ \\
\hline \multirow{2}{*}{ Keyhole } & Shock Velocities & $\pm 2 \%$ & $\pm 1.5-2 \%$ & $\pm 5 \%$ & $\pm 5 \%$ \\
\hline & Shock Merge Depths & $\pm 4 \mu \mathrm{m}$ & $\pm 2-5 \mu \mathrm{m}$ & $\pm 6 \mu \mathrm{m}$ & $\pm 9 \mu \mathrm{m}$ \\
\hline \multirow{4}{*}{$\begin{array}{l}\text { Backlit } \\
\text { Capsule }\end{array}$} & Velocity (Gated) & $\pm 5 \%$ & $\pm \mathbf{5} \%$ & $\pm 2 \%$ & $\pm 5 \%$ \\
\hline & Velocity (Streaked) & $\pm 1.5 \%$ & $\pm 3 \%$ & $\pm 2 \%$ & $\pm 4 \%$ \\
\hline & Mass Remaining & $\pm 1.5 \%$ & $\pm 1 \%$ & $\pm 1.5 \%$ & $\pm 1.5 \%$ \\
\hline & Hydroinstability GF & $\pm 20 \%$ & Designed & $\pm 20 \%$ & \\
\hline \multirow{6}{*}{$\begin{array}{l}\text { Symmetry } \\
\text { Capsule }\end{array}$} & Hot Spot Symmetry rms & $\pm 5 \mu \mathrm{m}$ & $\pm 3 \mu \mathrm{m}$ & $\pm 5 \mu \mathrm{m}$ & $\pm 5 \mu \mathrm{m}$ \\
\hline & Peak Drive (Dante) & $\pm \mathbf{5} \%$ & $\pm \mathbf{3} \%$ & $\pm 5 \%$ & $\pm \mathbf{5} \%$ \\
\hline & X-ray Bangtime & $\pm 50 \mathrm{ps}$ & $\pm 30 \mathrm{ps}$ & $\pm 50 \mathrm{ps}$ & $\pm 50 \mathrm{ps}, \mathrm{SPBT}$ \\
\hline & Hot e- @ Capsule & $\pm 50 \mathrm{~J}$ & $\pm 50 \mathrm{~J}$ & $<100 \mathrm{~J}$ & $<100 \mathrm{~J}$ \\
\hline & X-ray preheat fraction & $\pm 10 \%$ & $\pm 7 \%$ & $\pm 10 \%$ & $\pm 7 \%$ \\
\hline & Hot spot mix (ng of Ge) & $\pm 30 \mathrm{ng}$ & $\pm 20 \mathrm{ng}$ & $\pm 30 \mathrm{ng}$ & $\pm 30 \mathrm{ng}$ \\
\hline \multirow{8}{*}{$\begin{array}{l}\text { Cryo- } \\
\text { Layered } \\
\text { Capsule } \\
\text { (THD and } \\
\text { DT) }\end{array}$} & Yield (nToF, Activation) & $\pm 5 \%$ & $\pm 5 \%$ & $\pm 10 \%$ & $\pm 10 \%$ \\
\hline & Fuel pr (MRS) & $\pm 10 \%$ & $\pm 8 \%$ & $\pm 10 \%$ & $\pm 8 \%$ \\
\hline & Tion (nToF) & $\pm 10 \%$ & $\pm 7 \%$ & $\pm 10 \%$ & Bulk motion \\
\hline & Nuclear Bangtime (GRH) & $\pm 50 \mathrm{ps}$ & $\pm 20 \mathrm{ps}$ & $\pm 50 \mathrm{ps}$ & $\pm 30 \mathrm{ps}$ \\
\hline & Nuclear Burn Duration & $\pm 30 \mathrm{ps}$ & $\pm 30 \mathrm{ps}$ & $\pm 30 \mathrm{ps}$ & \pm 30 ps \\
\hline & HS radius $(\mathrm{P} 0)(\mathrm{NI})$ & $\pm 5 \mu \mathrm{m}$ & $\pm 3 \mu \mathrm{m}$ & $\pm 5 \mu \mathrm{m}$ & $\pm 3 \mu \mathrm{m}$ \\
\hline & HS symmetry rms (NI) & $\pm 5 \mu \mathrm{m}$ & $\pm 5 \mu \mathrm{m}$ & $\pm 7 \mu \mathrm{m}$ & $\pm 5 \mu \mathrm{m}$ \\
\hline & Fuel pr rms (CR) & $\pm 10 \%$ & $\pm 15 \%$, OMEGA & $\pm 10 \%$ & $\pm 15 \%$ \\
\hline
\end{tabular}

\section{Hohlraums with Gas-Filled Symmetry Capsules}

A picture of a Symcap target is shown in Figure 3-5. In Symcaps, the cryogenic fuel layer is replaced with an equivalent mass of ablator material. These capsules are designed to have a hydrodynamic response to long wavelength radiation asymmetry very similar to that of a cryogenic-layered target, but they are simpler to field [19-22]. They are also designed for a convergence ratio of 20-25, about half that of cryogenic layered targets, by having an initial gas fill of $\sim 6 \mathrm{mg} / \mathrm{cc}$ of $\mathrm{D}^{3} \mathrm{He}$. 


\section{National Ignition Campaign Program Completion Report}

Symcaps are used to establish whether:

1. The required peak radiation temperature can be achieved consistent with specifications on the radiation asymmetry;

2. The level and variability of scattered laser light is acceptable; and

3. The hot electron production from LPI effects and the level of hot electrons transported to the capsule are at acceptable levels.

Symcap targets are used to set beam smoothing characteristics of the laser, including smoothing by spectral dispersion and polarization smoothing, as well to set the hohlraum gas fill and the spot size to maximize coupling and minimize LPI effects. Symcaps are used to set the relative power in the beams and the wavelength separation between cones required to obtain long-wavelength radiation symmetry integrated over the pulse. Also obtained from these targets is the spectrum of the $\mathrm{x}$-rays in the hohlraum - in particular, the fraction of $x$-ray energy above $1.8 \mathrm{keV}$ that is responsible for preheat of the ablator at the fuel-ablator interface. This measurement is used to set the ablator dopant levels

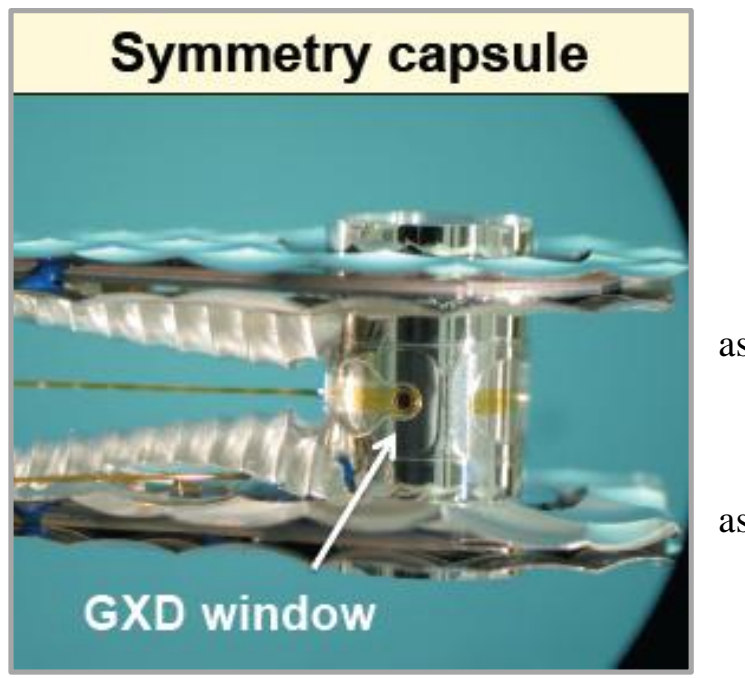

Figure 3-5. Picture of a Symcap target with a $400 \mu \mathrm{m}$ diameter $\mathrm{CH}$-filled diagnostic window on the hohlraum at midplane. as needed to control the Atwood number at the fuel-ablator interface. The hohlraum length is adjusted to optimize the $\mathrm{P} 4$ mode asymmetry in the radiation drive.

A wide range of diagnostic measurements provide data to assess these performance parameters. See I.5, Target Diagnostics and Experimental Systems, for more information on NIC diagnostics.

\section{Re-Emit Capsules}

Figure 3-6 is a picture of a Re-emit target. Re-emit targets are used to optimize symmetry during the first $2 \mathrm{~ns}$ of the pulse by imaging soft x-ray reemission from a bismuth-coated sphere, which replaces the standard $\mathrm{CH}$ capsule [23]. For the first couple of nanoseconds, the plasma conditions and the resultant beam propagation are the same for both capsules, but for longer times, ablation from the $\mathrm{CH}$ capsule produces plasma conditions in the hohlraum that differ from those in a hohlraum with a bismuth capsule.

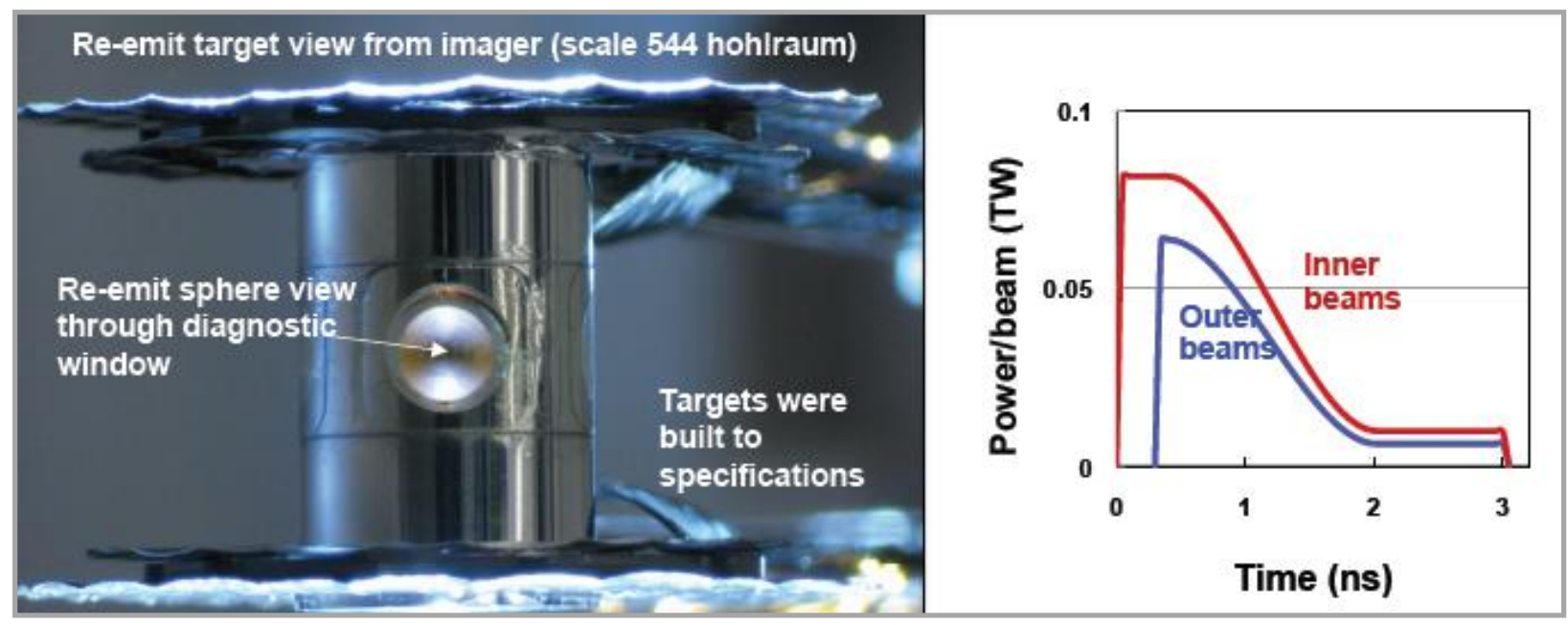

Figure 3-6. Picture of the Re-emit target and a typical initial 3 ns of pulse used in experiments. 


\section{National Ignition Campaign Program Completion Report}

\section{3. "Keyhole" Targets}

These targets have a cone inserted through the side of the hohlraum wall and into the capsule that is filled with liquid deuterium (surrogate for frozen DT) so that an optical interferometer (VISAR) [24] can see the inside of the shell. Keyhole targets measure the timing and strength of shocks launched in the ablator [25-27]. They also set the key parameters of the pulse shape - the power levels of the initial picket and the trough following the first picket; the power levels of the second and third shocks; the timing of the second, third, and fourth shocks; and the rate of rise of the radiation temperature during the fourth pulse.

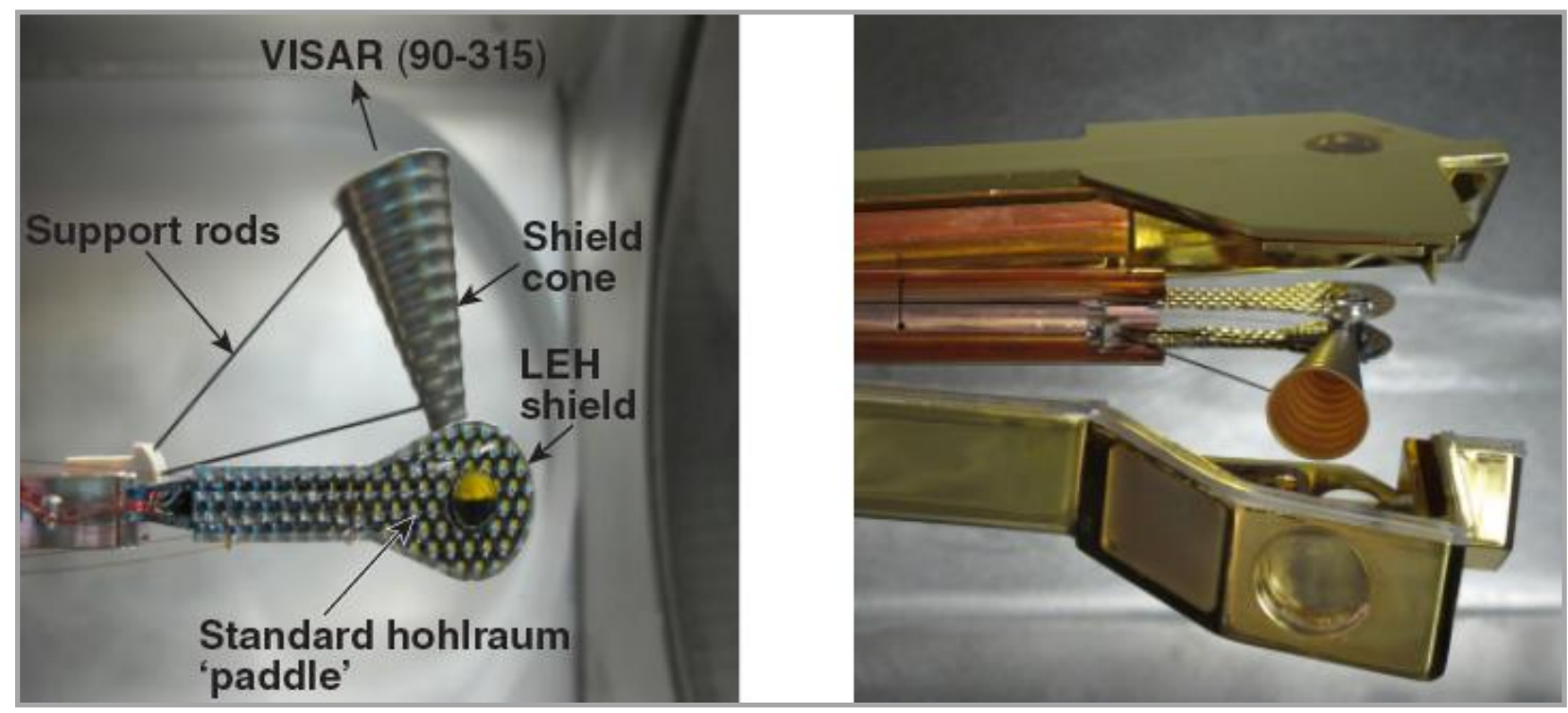

Figure 3-7. (Left) Picture of keyhole target with cone shield. (Right) Picture of keyhole target inside partially open custom shroud with VISAR window access.

If hot electron preheat effects from LPI meet specification, shock mistiming is the dominant source of entropy generation in the fuel. Initial keyhole experiments carried out 2010 had a single straight through line of sight through the cone that was pointed toward the waist of the hohlraum. A "mirrored" keyhole was introduced in 2011. The mirror allows the VISAR diagnostic to look both at the pole and waist of the capsule in a single shock. With the mirrored keyhole, the symmetry of shocks on the capsule as well as the strength and timing can be optimized. Pictures of the keyhole target used to set the shock timing and merge depth are shown in Figure 3-7.

\section{Convergent Ablator Target}

The ConA target can be either a Symcap or a cryogenic layered target, with a slot at the waist of the hohlraum and a backlighter illuminated by two quads of NIF beams. ConA targets are used to radiograph the position of the imploding shell radius versus time and the optical depth of the mass remaining in the ablator as it implodes $[28,29]$. This measurement is used to adjust the laser peak power and the shell thickness so that the implosion has both the required velocity and the required residual mass of ablator. The peak power and shell thickness, along with the dopant level in the ablator, must be adjusted to optimize the tradeoff between implosion velocity and mix. The position of the shell and its optical depth as it implodes can be obtained

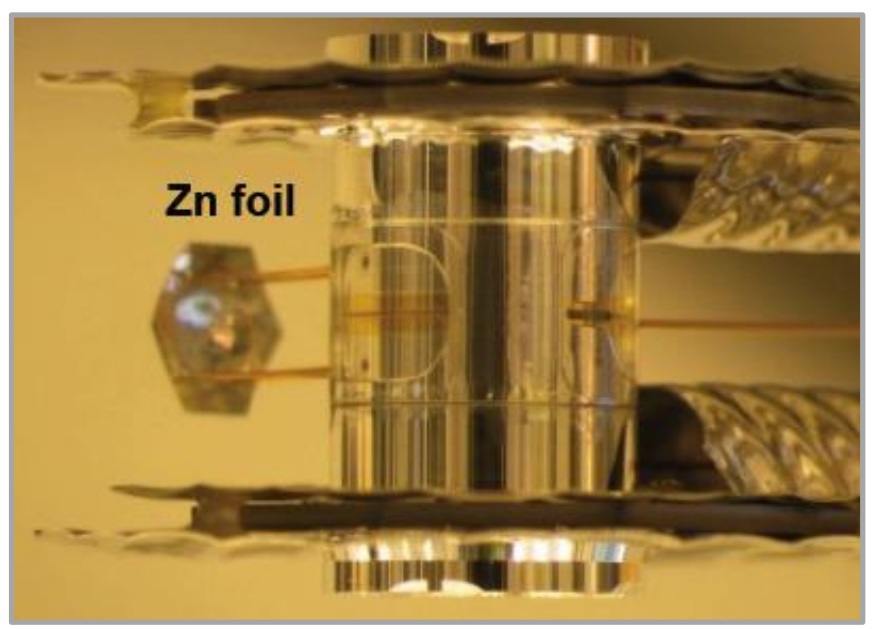

Figure 3-8. The ConA hohlraum with a Zn backlighter foil suspended at the midplane at $3 \mathrm{~mm}$ from the hohlraum wall. 


\section{National Ignition Campaign Program Completion Report}

either with a framed imager or a streak camera [30].

Figure 3-8 shows a picture of the target used to measure the trajectory, velocity, mass remaining, and thickness of the imploding ablator [31]. X-ray area backlighting was used by redirecting two of the $50^{\circ}$ drive quads, focused by CPPs to $1-3 \times 10^{15} \mathrm{~W} / \mathrm{cm}^{2}$, to a thin $(5-15 \mu \mathrm{m})$ mid-Z foil driven at peak power (8-9 TW/quad). This technique creates helium-like 2:1 resonance lines that cast a shadow of the capsule limb in-flight with up to 2 optical depths of contrast that are imaged with 10-25 $\mu \mathrm{m}$ wide slits or pinholes at 9-12x magnification.

ConA experiments prior to March 2012 used a framing camera that provided a sequence of snapshots of the capsule radius and optical depth versus time. Since March 2012, a streak camera has been used to provide a continuous record of radius and shell optical depth versus time. This also allows for increasing temporal resolution from 90 ps to below $30 \mathrm{ps}$, hence reducing motional blurring from $25 \mu \mathrm{m}$ to below 10 $\mu \mathrm{m}$ for more accurate late time shell density profile measurements.

A variant of the ConA target uses a 10-20 keV backlighter and a pinhole instead of a slit to obtain a sequence of 2D images of the optical depth of the imploding shell as a function of time. [32, 33] With 2D radiography, it is possible to obtain information about the spatial variation in the location of the shell as well as variations in the optical depth of the remaining mass.

\section{Mix Caps}

Mix Caps are a variant of Symcap with engineered surface features. The variation in optical depth of these features can be imaged during an implosion using a 5-10 keV x-ray backlighter $[34,35]$. The growth of variations in the optical depth is used to obtain hydrodynamic instability growth rates. The smaller the initial perturbation, the greater the inherent sensitivity $[36,37]$ to the high ablation front linear growth factors (500-1000) expected on NIF implosions. Mix depth is a nearly linear product of growth multiplied by the amplitude of surface features on ICF capsules. Measurement of this growth determines the level of surface roughness and the level of mass ablated required to keep mix at acceptable levels. Additional targets, which would use spectroscopy [38] or radiochemistry [39] to more directly measure mix at the fuel ablator interface, or deep within the hot spot, are being evaluated.

\section{Compton Radiography}

Information about areal density and shape of the dense cold fuel surrounding the hot spot can be obtained by active probing using an external source of hard x-rays. High-energy x-ray images [40] can be obtained using transmission Compton radiography where high-energy Compton scattering is used rather than traditional photo-absorption to cast a shadow of the imploding capsule. For photon energies of 50-200 $\mathrm{keV}$, the Compton scattering cross section is largely independent of photon energy. As a consequence, the optical depth of the fuel of an ICF target shows a plateau above $\sim 50 \mathrm{keV}$ where the Compton scattering dominates. Because of the slow dependence of the Compton scattering cross section on the $\mathrm{x}$-ray photon energy, the areal density of the fuel can be inferred even using a polychromatic backlighter. A broadband bremsstrahlung emitting source is adequate, and the energies of the x-ray photons can be selected by a combination of a high-pass filter and the detector response to optimize signal to background. The accuracy of measurements of fuel areal density depends mainly on the signal-to-noise ratio and on the contrast of the recorded radiograph. In the current implementation, the backlighting source is produced by irradiating Au wires with the UV beams from two tightly focused $8 \mathrm{TW}$ quads. A gated framing camera provides adequate 70 ps temporal resolution. When the NIF Advanced Radiographic Capability (ARC) is available, the $10 \mathrm{ps}, 1 \mathrm{~kJ} / \mathrm{beam}$ ARC pulses will be used to irradiate the Au micro-wires. Accuracies of between 7 and $10 \%$ for measurements of limb-averaged $\rho$ are estimated when long-pulse NIF UV beams are used to generate the backlighter and about $2 \%$ when using NIF-ARC.

\section{Cryogenic Layered Implosions}

The results of the implosion optimization campaign using the surrogate targets, discussed above, are used to set most details of the target and laser pulse for cryogenic layered capsule experiments, as discussed shortly after the first round of optimization by Glenzer et al. [41] Nevertheless, some physics issues that 


\section{National Ignition Campaign Program Completion Report}

result from the layer itself can only be addressed with cryogenic layered target experiments; these include instability growth at the hot spot/main fuel interface and at the ablator/main fuel interface as well as surrogacy issues arising from differences between the tuning targets and cryogenic layered targets. The shape of the hot spot, spatial distribution of compressed fuel in the main DT layer, mass remaining, velocity, and velocity profile of the imploding fuel layer can produce slight variations between a surrogate and a layered target.

Cryogenic layered targets can be designed with varying layer compositions, from layers for low yield using a fuel layer composition consisting mainly of tritium and hydrogen with only a few percent deuterium (referred to as THD layers), to 50:50 DT layers. The ratio between hydrogen, deuterium, and tritium in the reduced deuterium targets is chosen to maintain a density in the frozen fuel layer equal to that of 50:50 DT layers in order to retain surrogacy for hydrodynamic instability at the fuel-ablator interface. These cryogenic layered targets and their expected performance have been discussed extensively in an article by Edwards et al. [10], The full array of diagnostics needed to optimize the fuel assembly can be used with cryogenic layered THD targets designed for low yield, before shifting to 50:50 DT layers to obtain higher yields and ultimately to achieve ignition.

A central goal of the THD experiments is to optimize the tradeoff between velocity and mix in the ITF. Achieving higher velocity requires ablating more mass. With less mass remaining, instabilities, which initially grow on the ablation front, imprint on the ablator-fuel interface and provide a larger seed for growth at that interface. Since ITF depends so strongly on velocity, ITF will increase until mix begins to penetrate a significant fraction of the cryogenic fuel layer, or until the penetration of mass from isolated defects in the ablator starts to significantly affect the hot spot, as described above.

The nominal values of velocity and mix penetration are chosen based on extensive computational studies, as discussed by Haan et al. [3] The ablated mass and velocity can be adjusted by varying the peak laser power until optimal performance is found. If this velocity is below the velocity needed for ignition, we can either reduce the size of perturbations by improving target fabrication, work to reduce the growth rates that are dependent on the capsule dopant profile and the laser pulse shape, or increase the laser power and energy in order to implode thicker shells and/or thicker fuel layers to higher velocity. Calculations indicate that growth during the initial picket and the several nanosecond trough that follows can vary by factors of $2-4$, depending on details of the pulse. The point design is optimized to minimize this growth, but the optimum is uncertain because of equation of state and radiation transport effects. Hydrodynamic instability growth during the foot of the pulse can be measured using the Mix Cap targets discussed above and optimized by modifying the initial picket and the subsequent trough.

Mixing of the ablator into the fuel can result from instabilities at the ablation front and at the fuel-ablator interface. Mix becomes more of an issue as the implosion is optimized to produce higher convergence and higher compression. Mix signatures include a reduced yield and ion temperature due to radiative cooling and high $\mathrm{x}$-ray brightness from the hot core due to the higher $\mathrm{Z}$ ablator mixing into the compressed fuel. Line emission from the hot spot arising from mixing of dopants in the ablator into the fuel is also used to quantify mix [42].

The effect of mix can be seen in Figure 3-9 where the neutron yield is plotted versus the inferred ablator mass remaining at peak velocity for the recent high areal density implosions. The remaining mass is a measure of the amount of material that instabilities at the ablation front must penetrate to mix ablator into the hot fuel. Target yields are significantly reduced for implosions with less than $0.35-0.4 \mathrm{mg}$ of remaining mass. This is about $12 \%$ of the initial ablator mass. X-ray brightness from total capsule x-ray emission and spectroscopy measurements also confirmed this trend. The red dashed line in Figure 3-9 is the remaining mass in the ignition point design. A fairly sharp performance boundary referred to as a "mix cliff" occurs at a remaining mass $\sim 30-40 \%$ greater than that used in the point design. Targets designed to maintain the larger remaining mass require thicker ablators and hence more energy to accelerate the shell to the same velocity. 


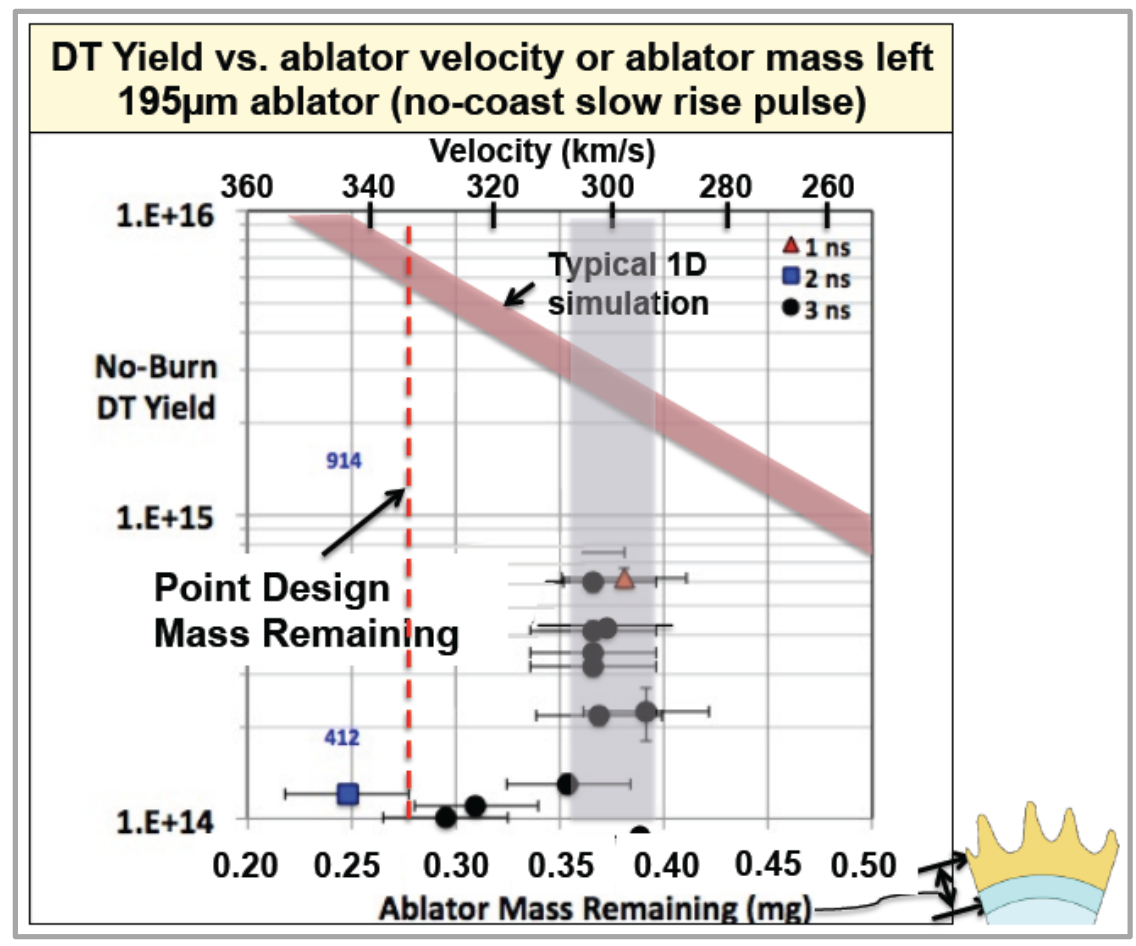

Figure 3-9. A "mix cliff" is found with $\sim 30-40 \%$ more ablator mass remaining than that for the point design calculation. Increasing the yield by a factor of 3-5 at the current velocity will be a focus of upcoming experiments.

There are various possible explanations for the required increase in remaining mass in the current experiments. These include unmeasured sources of perturbation, growth rates that exceed those currently calculated, and long wavelength variations in the fuel thickness that make it easier for the ablator to penetrate through the resulting thin region. Experiments are being developed to test possible causes. Improvements to the target roughness and laser power balance that could reduce the required remaining mass are also being pursued.

In addition to the mix cliff, Figure 3-9 shows that yields in present experiments are $\sim 5 \mathrm{x}$ lower than calculated 1D yields. The solid red band shows the predictions of yields from 1D- simulations as a function of remaining mass. The simulations are for typical implosions with this ablator thickness and target size and have been adjusted to match the observed shock timing and shell trajectories as describe in Figure 3-10. Understanding this difference and reducing the magnitude of the difference between experiments and simulations is important for ignition target performance. The simulations show that if the targets were performing at the calculated 1D levels of yield, alpha deposition would be significantly enhancing the yield even at these lower velocities.

An alternate metric for plotting the performance of capsules near the mix cliff is to plot the ion temperature and yield versus the shell thickness as shown in Figure 3-10. This is motivated by the dependence of the RT ablation front instability growth exponent fed through on acceleration to the ablator-DT fuel interface [43].

A standardized approach has been developed to calculate 1D drives used in predicting the expected performance of cryogenic layered implosions. The radiation drive is adjusted until the calculations match the keyhole VISAR shock timing data and the convergent ablator radius versus time. The chart on the left in Figure 3-11 shows the calculated drive in an integrated hohlraum and capsule simulation, the drive as measured by the Dante instrument, and the adjusted drive used to match the VISAR and ConA data. The peak drive must be adjusted downward by about $15 \%$ relative to the standard High Flux Model 


\section{National Ignition Campaign Program Completion Report}

calculation of the hohlraum to match the measured implosion velocity. Analysis of the Dante experimental data indicates that the x-ray drive predicted by the standard High Flux model is higher than the experimental drive by of order $15 \%$. Additional multipliers for earlier in the pulse are also required to match the VISAR shock timing data. When the drive is adjusted to match the shock timing data and the shell radius versus time in Figure 3-11, the remaining mass and shell thickness are also matched within the error bars, though shell thickness is always somewhat greater than simulated. The table in Figure 3-12 shows that predictions with these adjusted drives match much of the observed core size and shape and ion temperature data for cryogenic layered implosions but typically overestimate the hot spot density by $2 \mathrm{x}$ and the yield by factors of several.

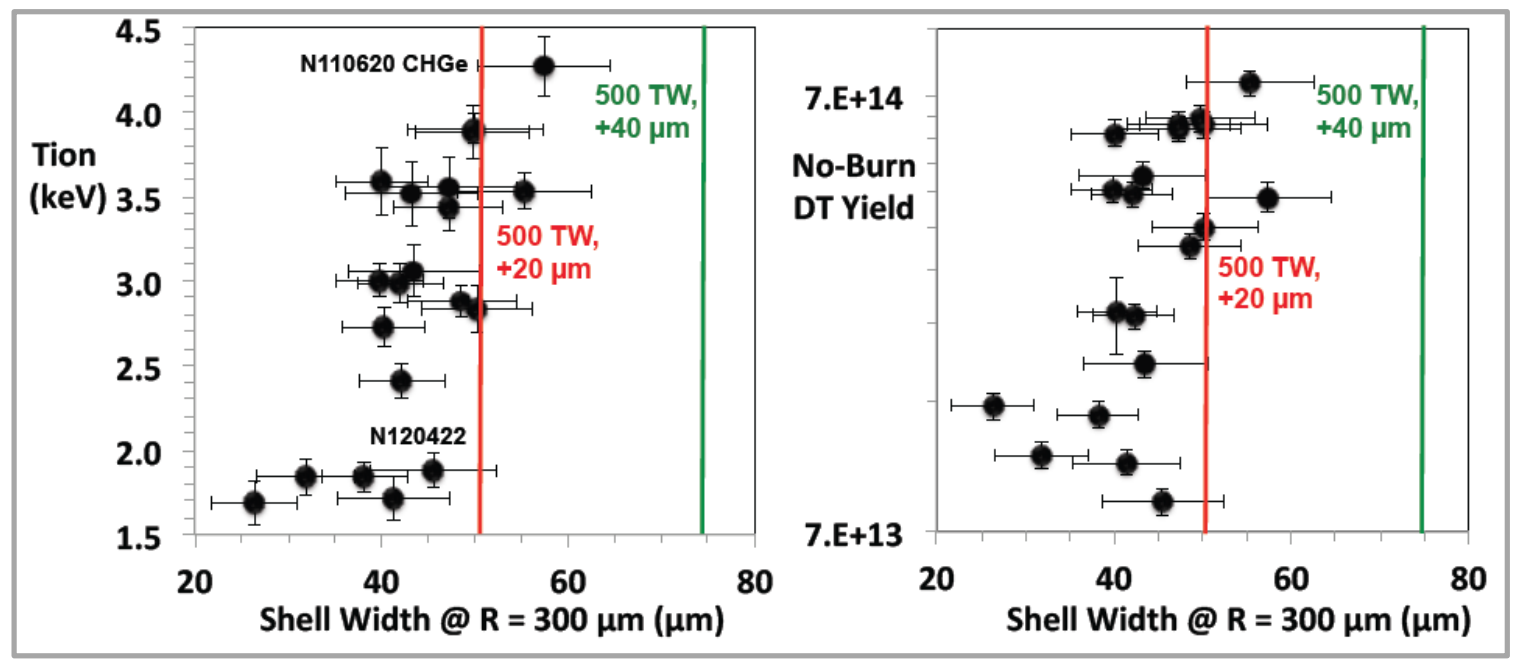

Figure 3-10. $T_{\text {ion }}$ and yield vs. ablator thickness at $R=300 \mu \mathrm{m}$ for all $>1.4 \mathrm{MJ}$ DT and THD shots since June 2011. The yield and $T_{i o n}$ for both low-coast and coasting shots show a common value for the shell thickness at the mix cliff.

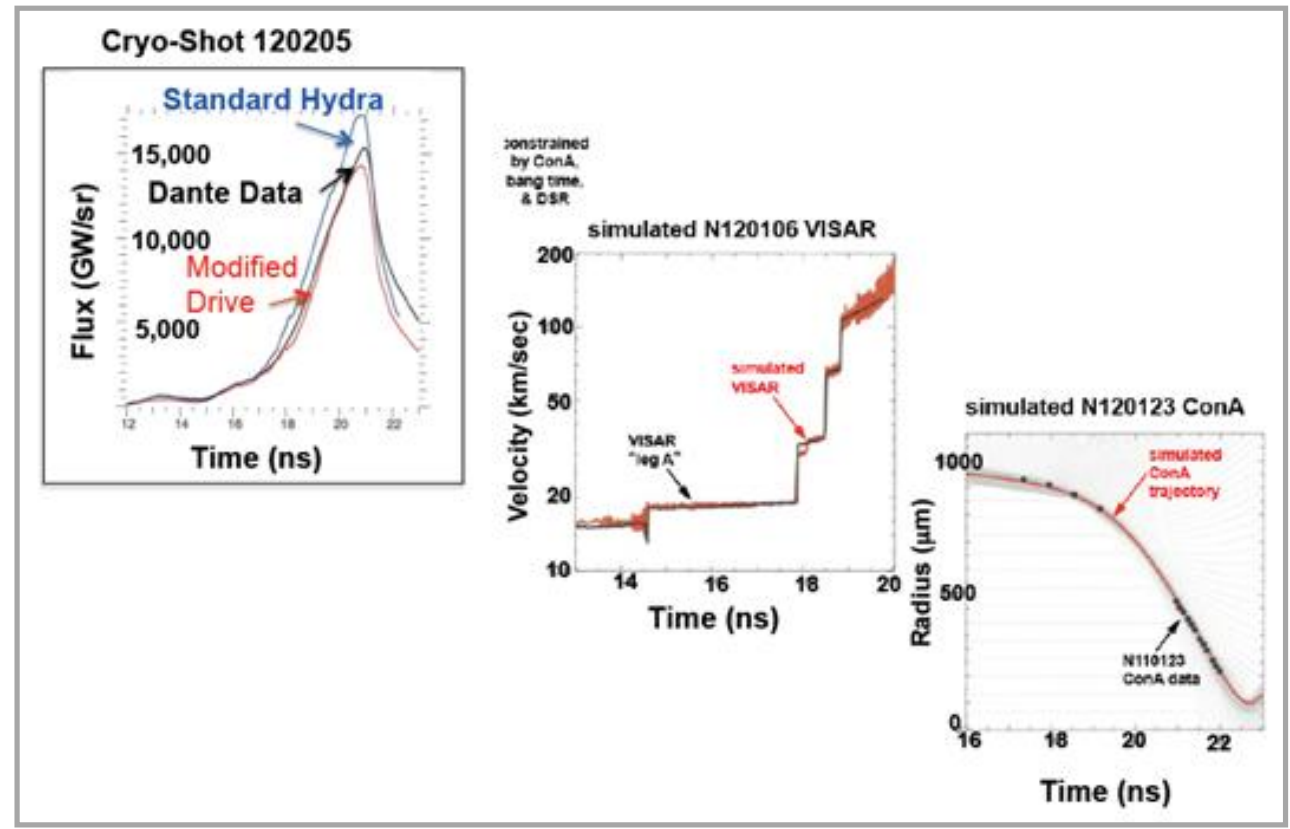

Figure 3-11. (Left) X-ray flux vs. time through LEH: data (black), standard HYDRA simulation (blue), and modified drive (red). (Middle) Simulated (red) and measured (black) leading shock velocity vs. time. (Right) Simulated (red curve) and measured (black dots) ConA radius vs. time. These charts show how the radiation drive is modified so that the calculation matches the VISAR and ConA data. This approach has enabled an exploration of the differences between the models and data throughout the implosion process and a correction for those differences as the campaign progressed. 


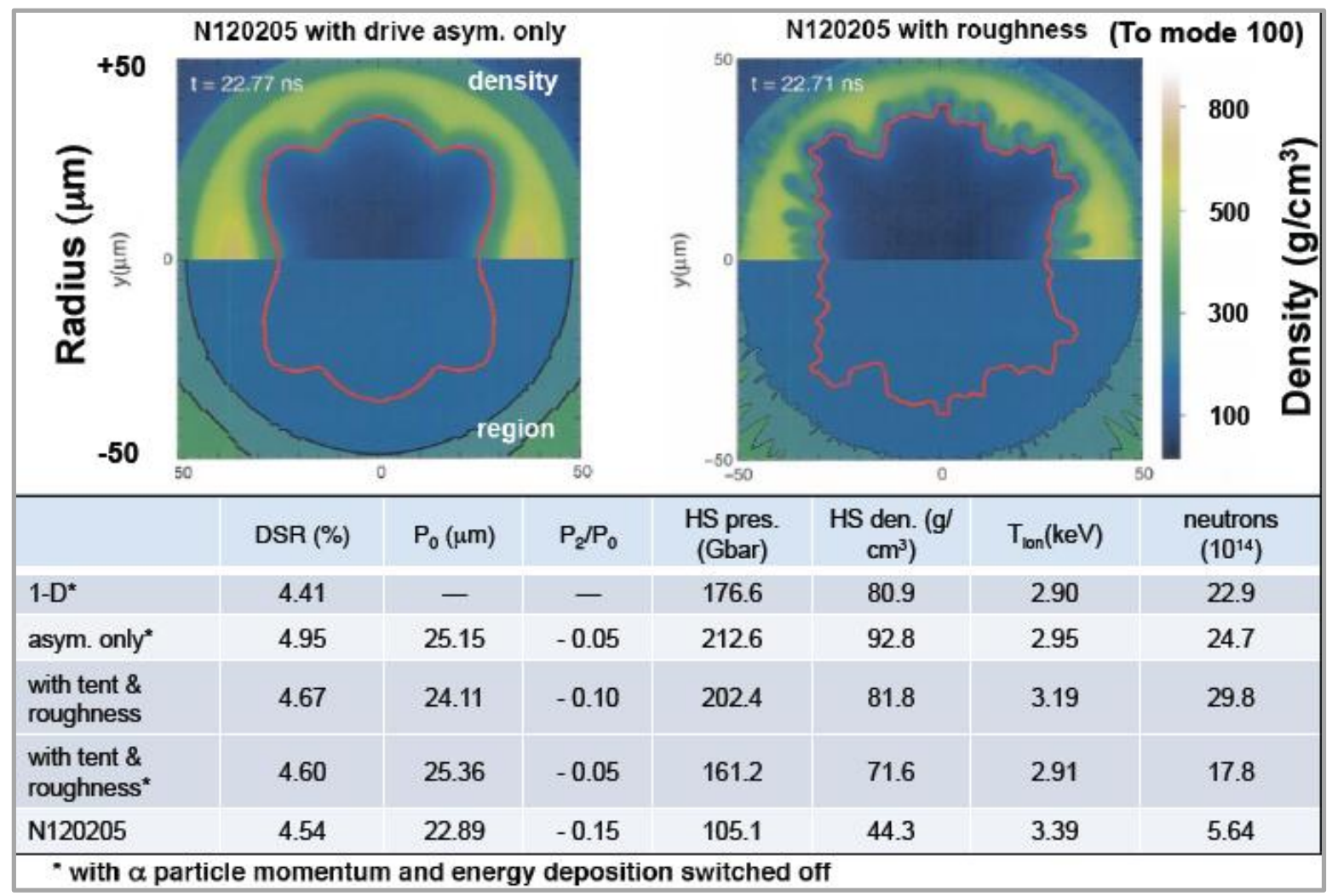

Figure 3-12. Simulated density cross-sections with drive asymmetry or roughness (top row) for cryogenicallylayered DT implosions shot N120205. Table below compares simulated observables and data.

A potential source of the yield deficit in implosions without obvious mix into the hot spot could still be the result of ablator mixing into the main fuel. However, the reduced yield could also result from lowmode asymmetry of the cold fuel and ablator. Measurements of the isotropy of the neutron yield, the neutron downscatter spectra, and images of the downscattered neutrons suggest that the cold fuel likely has a higher areal density at the poles of the implosion along the axis of the hohlraum than around the equator. Another hypothesis is that mix is occurring preferentially at thin spots of the ablator and/or fuel due to non-uniformities caused, for example, by residual symmetry swings. The nuclear measurements indicate that the main fuel can have quite large $\rho$ r variations even when the hot spot shape is quite round. By implementing 2D $10 \mathrm{keV}$ and $100 \mathrm{keV} \mathrm{CR}$ (discussed earlier), the in-flight and compressed ablator and fuel can be checked for distortions and thin spots that could enhance mix feed-through locally and hence increase the required spatially averaged minimum mass remaining.

In addition to minimizing these low-mode asymmetries, further optimization of the peak power pulse shape beyond the simple variations in the rise times tested to date may also be required to achieve increased hot spot density and improved yields predicted by integrated simulations [44]. Besides varying the peak power pulse shape, the level and length of the foot can affect mix. Specifically, seeding of the RT instability by Richtmyer-Meshkov growth during the initial shock traversal phase might be reduced by factors of 2 by altering the trough power and length, which can be tested by x-ray radiography of large pre-imposed perturbations [45] driven by just the foot of the NIC pulse. Finally, designs with higher foots and shorter pulses to put the capsule on a higher in-flight adiabat [46] might be used to reduce mix from increased ablative stabilization because they have a lower in-flight shell density and reduced convergence. 


\section{National Ignition Campaign Program Completion Report}

\section{E. Use of ITFX and Fuel Stagnation Pressure to Assess Progress toward Achieving the Requisite Fuel Assembly}

Diagnostics developed to characterize the state of the imploded fuel in the cryogenic layered targets are used to address the third key question of the ignition campaign: How is progress toward the achievement of those conditions gauged as the ignition campaign progresses? As discussed at length in a paper by Edwards et al. [10], a THD measureable parameter given by:

$$
\operatorname{ITFX}(\operatorname{Re} v 5 C H)=\left(\frac{0.17 m g}{m_{D T}}\right)\left(\frac{Y}{1.8 \times 10^{14}}\right)\left(\frac{d s f}{0.07}\right)^{2.3}
$$

is found to be a good predictor of DT performance. The yield Y in the ITFX formula is the measured neutron primary yield (defined as the integral between 13 and $15 \mathrm{MeV}$ ) and $\mathrm{m}_{\mathrm{DT}}$ is the fuel mass. The DSF is the measured neutron downscattered fraction (defined as the number of neutrons between 10 and $12 \mathrm{MeV}$ expressed as a fraction of those between 13 and $15 \mathrm{MeV}$ ). At ITFX $=1$, as for ITF $=1$, the probability of achieving a yield $\geq 1 \mathrm{MJ}$ is $50 \%$. The constants in ITFX above are the mean values for yield and DSF for ITFX $=1$ for the optimized CH capsule discussed above that is the baseline for the NIC. The value for the yield depends on the $\mathrm{D}$ fraction and the number given, $1.8 \times 10^{14}$, is appropriate for $2 \% \mathrm{D}$. The DSF of 0.07 for neutrons between 10 and $12 \mathrm{MeV}$ corresponds to a DT fuel $\rho r \sim 1.4 \mathrm{~g} / \mathrm{cm}^{2}$. There is an additional $\mathrm{\rho r} \sim 0.4 \mathrm{~g} / \mathrm{cm}^{2}$ of $\mathrm{CH}$ from the remaining ablator.

An alternate and complementary approach to ITFX for assessing progress toward the achievement of ignition conditions uses a synthesis of data obtained from the capsule implosions. The peak pressure achieved in an implosion is a very good integral measure of implosion performance. THD implosions that have an ITFX 1 also have a peak pressure $\sim 350$ Gbar. For example, an implosion with a hot spot density that is a little over $125 \mathrm{~g} / \mathrm{cm}^{3}$ and a central $\mathrm{Ti} \sim 3.5 \mathrm{keV}$, typical of a capsule with ITFX 1, would have a pressure of $\sim 350$ Gbar. From a large ensemble of THD calculations in which ITF varies from about 0.04 to 6 , the central pressure at the time of peak neutron production rate is found to be given by:

$$
P_{\text {peak-burn }}(G B a r)=350 \pm 45(I T F X)^{0.35}
$$

Even though pressure is not measured directly, it can be estimated from a measurement of the size of the imploded fuel region, the measured ion temperature, and the yield. With adequate knowledge of the spatial temperature variation in the hot spot, which can be obtained from x-ray images of the hot spot at different photon energies, and assuming an isobaric implosion, it is possible to estimate the pressure in an implosion that quite accurately matches simulations. This type of analysis is being applied to both Symcap and THD implosions. Although ITFX is used as the primary measure of capsule performance, the estimated pressure provides complementary information.

Improved shock timing [47] along with extended pulses to maximize the imploding shell density prior to stagnation has resulted in two discrete jumps in fuel $\rho$, which has reached about $85 \%$ of the point design value. Progress toward ignition can be graphically represented as shown in the left-hand chart in Figure 313 , where cryogenic layered target neutron yield is plotted versus the DSF and where the contours drawn represent constant ITFX. The yields plotted are those arising from compression during the implosion process and do not include enhancements from alpha deposition; these enhancements become significant as the implosion quality approaches that required for ignition. As described above, this yield is adopted because it provides a metric for the underlying quality of the implosion needed to get into a regime where the alpha particle deposition becomes significant.

Progress in ITFX can be summarized by the following:

- Significant improvement in ITFX has been shown between the first cryogenic layered implosion in September 2010 and February 2011. This improvement, mostly in the neutron yield, was 


\section{National Ignition Campaign Program Completion Report}

realized by increasing the laser power and energy and performing initial shock tuning experiments. Target improvements were also realized by preventing frozen condensation on the LEH windows (see I.4.1, Target Development and Manufacturing).

- During 2011, ITFX was increased to $~ 0.08$. This was achieved through improved shock timing, implosion velocity (using silicon ablators), laser performance, and implosion symmetry.

- In 2012, the slope of the rise of the fourth pulse was decreased, producing an implosion with higher compression and lower adiabat. The length of the fourth pulse was extended to better maintain compression of the shell during final convergence, referred to as a "no-coast" pulse. This produced a higher DSR, or $\rho r$, resulting in an improved ITFX of $\sim 0.1$ at significantly lower velocities and decreased drive energy.

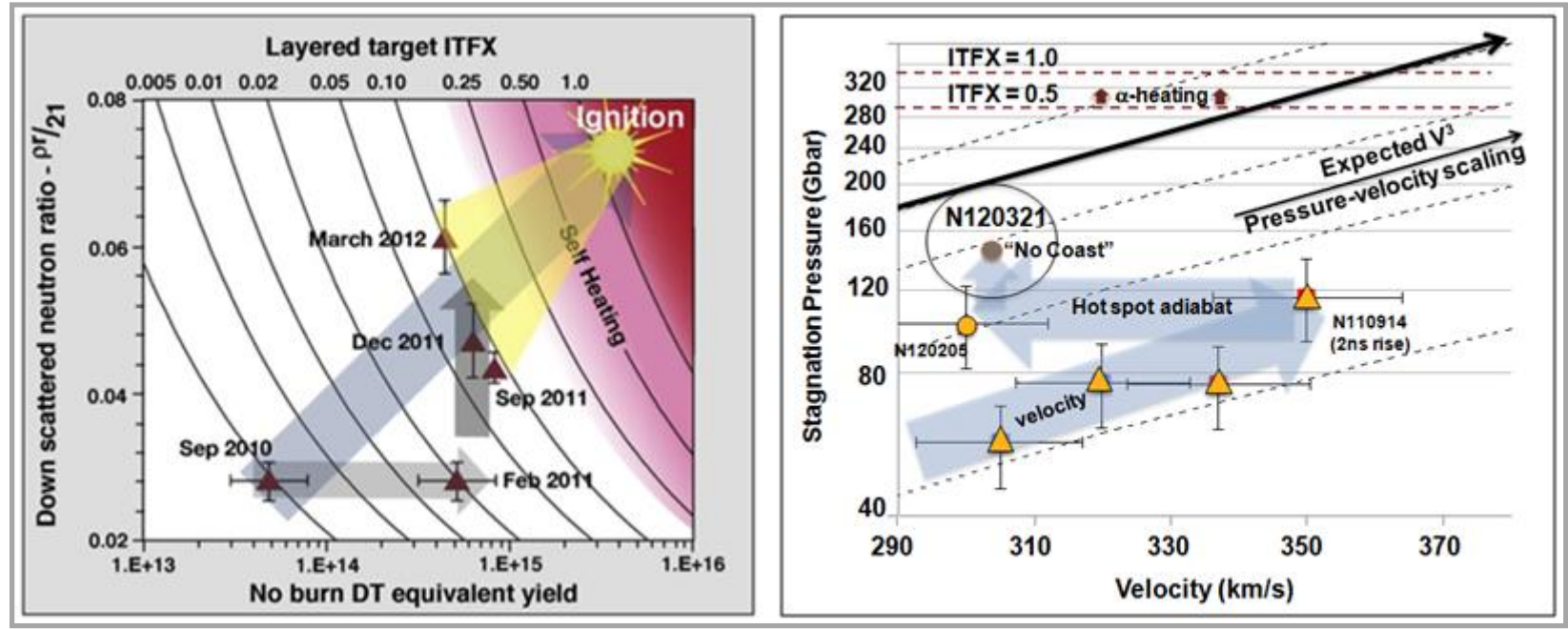

Figure 3-13. (Left) Peak values of ITFX are about $10 \%$ of that needed for ignition. The downscattered ratio, or the fuel $\rho r$, is now at about $85 \%$ of the point design value, but the yields must be increased $\sim 5 x$ to get into the alpha dominated regime and $\sim 10 x$ for ignition. (Right) Stagnation pressure vs. peak fuel velocity. Sloped lines are expected for scaling at given adiabat and dashed horizontal lines are ITFX values.

ITFX alone does not capture progress in the performance of recent layered implosions. Although ITFX increased by only about 25\% from September 2011 to March 2012, the present implosions provide a much better path to ignition:

- Present experiments produce higher compressed fuel densities and higher areal densities, $\sim 85 \%$ of the ignition point design.

- The pressure in the hot spot is $\sim 140$ Gbars, $\sim 40 \%$ of the ignition point design.

- These conditions were obtained using peak powers of $320 \mathrm{TW}$ with implosion velocity of $\sim 300 \mathrm{~km} / \mathrm{s}$, well below the NIF rated performance of $500 \mathrm{TW}$, substantially increasing the laser performance margin available for ignition.

The right side of Figure 3-13 shows that using "no coast" pulses, with extended duration at peak power to better maintain compression of the shell prior to final stagnation, the highest pressure to date ( 140 Gbar) has been achieved at a $50 \mathrm{~km} / \mathrm{s}$ lower velocity than the previous high pressure data obtained in September 2011.

In contrast, the best implosions in CY2011 achieved $\sim 65 \%$ of the areal density of the point design with an implosion velocity of $\sim 340 \mathrm{~km} / \mathrm{s}$ and $420 \mathrm{TW}$ of laser power. Also, these earlier implosions had lower peak hot spot pressures, $\sim 30 \%$ of the ignition pressure at ITFX $=1$. Since ITFX is a strong function of velocity and since peak velocities are expected to be limited to $\sim 370 \mathrm{~km} / \mathrm{sec}$, achieving the improved performance at a substantially lower velocity provides a better path to ignition. The right-hand chart in Figure 3-13 shows the improvements in peak fuel pressure versus implosion velocity. The challenge is to 


\section{National Ignition Campaign Program Completion Report}

increase the peak fuel stagnation pressure by about $50 \%$ at $300 \mathrm{~km} / \mathrm{s}$ and then scale present implosions to velocities of $\sim 370 \mathrm{~km} / \mathrm{s}$ using the increased laser power margin without getting significant mix.

\section{F. Direct-Drive Research within the National Ignition Campaign}

\section{Introduction}

LLE, the world leader in the research and development of direct-drive ignition, has lead NIC's directdrive ICF research efforts in support of NIC's indirect-drive ignition efforts in three primary ways:

- Development of diagnostics and diagnostic techniques to understand the performance of compressed cryogenic fuel. (See I.5, Target Diagnostics and Experimental Systems, for more on diagnostics for NIC.)

- Study of cryogenic target implosions under ignition-relevant conditions on the OMEGA laser, to validate models for compressed target conditions. The compressed target conditions are insensitive to whether the target is directly or indirectly-driven.

- Development of direct drive as a viable alternative to indirect-drive ignition, providing the potential for higher gains or more robust ignition. This includes the development a strategy for PD ignition that could be demonstrated with NIF enhancements [48].

\section{Ignition-Relevant Cryogenic Fuel Assemblies}

An important, but unanswered, question at the beginning of the NIC was whether the models for DT under extreme compression were valid. LLE's cryogenic target research was able to answer this question, thus removing this uncertainty in target performance. This section briefly describes measurements of high areal densities in OMEGA cryogenic target implosions that give confidence in models of the DT compressibility under ignition-relevant conditions. As described in more detail below, LLE uses a triple picket laser pulse to compress cryogenic targets; areal densities of up to $300 \mathrm{mg} / \mathrm{cm}^{2}$ have been demonstrated in OMEGA cryogenic target experiments [49, 50]. Figure 3-14 shows a comparison of the measured areal densities with the predictions of 1D simulations using the LILAC hydrodynamic code [51]. Good agreement has been observed, validating the properties of compressed DT under ignitionrelevant conditions. In the following section, a detailed description of the progress in the understanding of direct-drive target physics is provided.

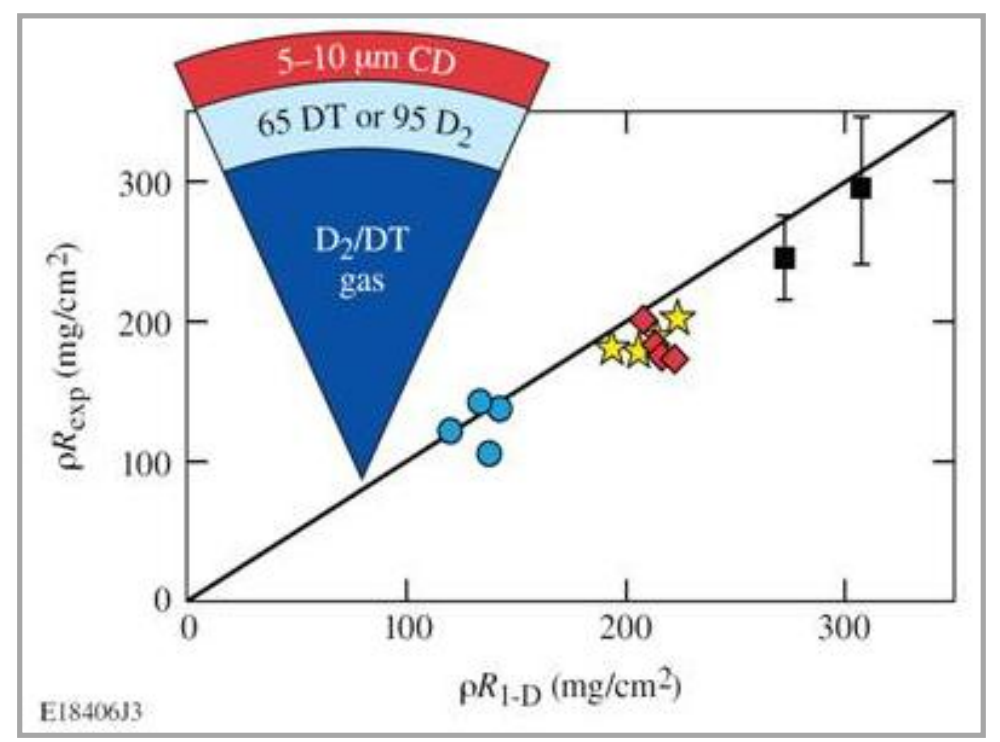

Figure 3-14. Comparison of measured and predicted compressed areal density in OMEGA cryogenic target implosions. 


\section{National Ignition Campaign Program Completion Report}

\section{LLE Progress on Direct-Drive Research}

Direct-drive ICF experiments at LLE's OMEGA Laser Facility [52, 53] have been used to help understand the underlying physics that feeds into the design of NIF ignition targets (both direct and indirect drive). Direct drive is predicted to couple 7-9 times more energy to the compressed capsule than indirect drive; thus, direct-drive ignition targets potentially could have higher gains or more margin than indirect-drive ones. Direct drive enables ignition-relevant compression conditions to be achieved on OMEGA even though the laser energy is $1 / 50$ th of that available on the NIF.

The high areal densities observed in OMEGA cryogenic target implosions led to the development of diagnostics that are used on the NIF to measure the compressed target performance of indirect-drive implosions. The directly driven exploding pusher platform has been transferred from OMEGA to the NIF to provide the neutron yields desired for activation and calibration of the NIF nuclear diagnostics [54]. DT neutron yields in the range of $10^{11}-10^{15}$ have been produced.

During the NIC, NIF was configured for the polar illumination that is required for indirect drivecylindrically, but not spherically symmetric. LLE has developed the PD concept to enable exploration of direct-drive ignition while NIF is configured for indirect drive [48]. During the NIC, LLE has made significant progress in improving the understanding and performance of symmetric cryogenic target implosions on OMEGA. Initial PD implosions of "exploding pusher" capsules have been performed on the NIF providing an initial study of PD symmetry [54]. A PD Execution Plan has been prepared that describes the requirements for the NIF laser to allow potentially uniform PD operations.

\section{a. Validation of Direct-Drive Physics on OMEGA}

The baseline symmetric-illumination ignition target design consists of a $1.5 \mathrm{MJ}$ multiple-picket laser pulse that generates four shock waves (similar to the NIF baseline indirect-drive design) and produces a $1 \mathrm{D}$ gain of $\sim 48$ [55]. Initial PD ignition designs have a predicted $2 \mathrm{D}$ gain of $\sim 32$, including all known sources of non-uniformities [56]. Verification of the physics basis of these simulations is a major thrust of implosion experiments on both OMEGA and the NIF. Many physics issues are being examined with symmetric beam irradiation on OMEGA. Cryogenic DT target experiments with symmetric irradiation have produced areal densities of $\sim 0.3 \mathrm{~g} / \mathrm{cm}^{2},[49,50]$ ion temperatures up to $3 \mathrm{keV}$, and neutron yields of up to $20 \%$ of the "clean" $1 \mathrm{D}$ predicted value.

LLE's plan for demonstrating PD ignition is based upon a large number of target shots on the OMEGA Laser Facility to validate the physics models used in multi-dimensional simulations codes, supported by a limited number of shots on the NIF to confirm the underlying physics at the larger energies and scales provided on the NIF. The main components of this multi-stage program are:

- Validating direct-drive physics models with symmetric cryogenic target implosions on OMEGA, including demonstrating performance that scales to ignition on the NIF.

- Extending these results to PD cryogenic target implosions on OMEGA [57].

- Demonstrating the technologies required for PD ignition on the NIF.

- Performing initial PD experiments on the NIF with indirect-drive smoothing (phase plates, etc.) that validate understanding in the higher energy and longer scale-length plasmas.

- Outfitting NIF with the phase plates and other beam smoothing capabilities required for PD ignition target designs, and demonstrating ignition.

As mentioned, direct drive is predicted to couple 7-9 times more energy to the compressed capsule than indirect drive for the same laser energy. This increased energy coupling potentially provides a large area in target design space to balance the requirements of minimizing the energy required for ignition [58, 59] with the need to ensure that the target is sufficiently stable to ignite. There are three primary parameters that determine the implosion performance: 


\section{National Ignition Campaign Program Completion Report}

- Target adiabat: Defined as the mass-averaged adiabat of the shell fraction contributing to the stagnation pressure,

$$
\text { adiabat }=\frac{\text { pressure }(\text { Mbar })}{2.2 \rho^{\frac{5}{3}}}
$$

- In-flight Aspect Ratio (IFAR): Defined as the ratio of $2 / 3$ of the implosion radius to $2 / 3$ of the shell thickness,

$$
\operatorname{IFAR}_{2 / 3}=\mathbf{R}_{2 / 3} / \rho_{2 / 3}
$$

- Implosion velocity: The implosion velocity determines the minimum energy required for ignition [59],

$$
E_{\min } \sim 1 /\left(V_{\text {imp }}\right)^{6}
$$

These three parameters determine the target stability and performance. The adiabat determines the target compressibility and the RT growth rate [60]. The in-flight aspect ratio determines the amplitude of the RT modulations that disrupt the implosion. The implosion velocity is determined by the acceleration of the target, which determines the number of RT growth factors.

Direct-drive cryogenic target experiments on OMEGA have been exploring the target performance as a function of these parameters. The implosions of $\sim 430$ $\mu \mathrm{m}$ diameter thin plastic ablators (5-12 $\mu \mathrm{m}$ thick) enclosing a DT ice layer $(50-90 \mu \mathrm{m})$ are driven with triple-picket pulses as shown in Figure 3-15. The target adiabat is varied by changing the spacing and power in the pickets and the step on the main pulse rise. The IFAR is varied through changes in the ablator and ice thicknesses, while the implosion velocity is varied through the total target mass and laser intensity. While these variables are not completely independent, they provide a convenient parameterization of target performance that provide the design space for ignition designs on the NIF.

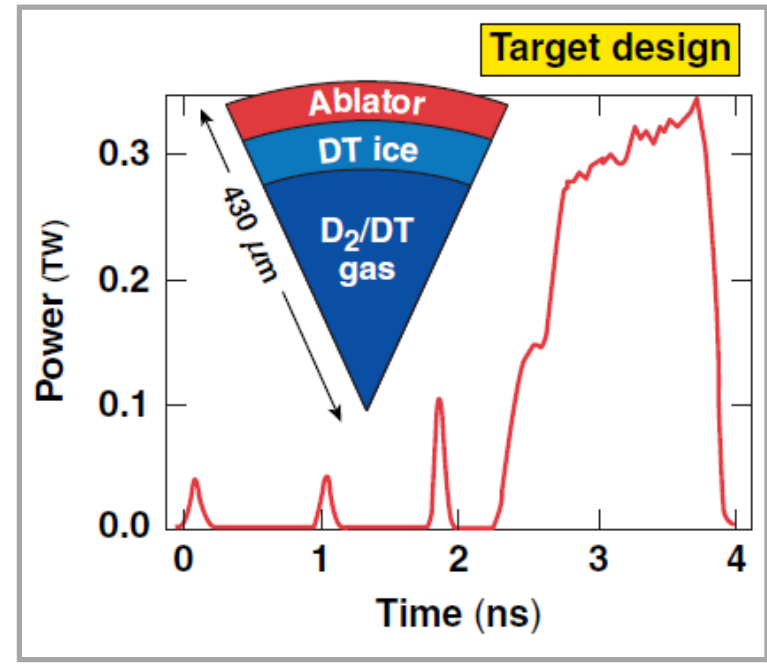

Figure 3-15. Schematic of OMEGA cryogenic target and typical pulse shape used to drive it.

\section{b. Symmetric Cryogenic Target Implosions on OMEGA}

The performance of direct-drive cryogenic target implosions is being systematically explored through variations of the target adiabat, IFAR, and implosion velocity, with the highest implosion velocities to date reaching approximately $3.6 \times 10^{7} \mathrm{~cm} / \mathrm{s}$. The performance is parameterized by comparing the measured neutron yield and areal densities to those predicted by $1 \mathrm{D}$ simulations using the LILAC hydrodynamic code [51]. LILAC includes the best current models for nonlocal electron transport [61] and crossed-beam energy transfer [62-64]. These models agree with a wide variety of experiments and are used without "adjustable knobs." The left panel of Figure 3-16 shows the ratio of the neutron yield to the 1D prediction yield over clean (YOC) as a function of the calculated target adiabat. The data is separated into those with IFARs below and above 22. The right panel shows a map of the YOC as a function of adiabat and IFAR. The black points are the results of OMEGA implosions while the color shows the measured YOC. 


\section{National Ignition Campaign Program Completion Report}

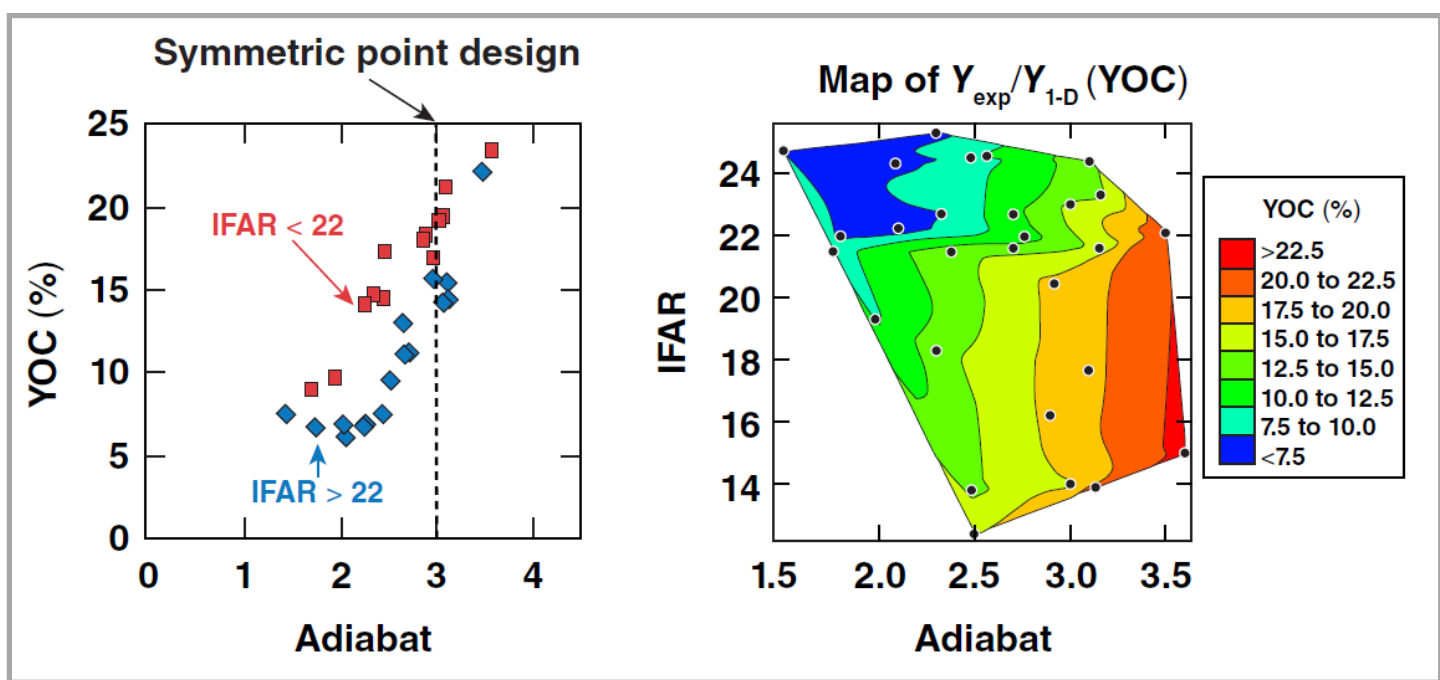

Figure 3-16. Experimental yield over clean (YOC) as a function of target adiabat (left panel) for OMEGA cryogenic target implosions. The data is separated by the IFAR (red points IFAR <22, blue points higher IFAR). The vertical dashed lines show the values of adiabat for the symmetric direct-drive ignition design. The right panel shows a map of the YOC as a function of adiabat and IFAR. The black points are the results of OMEGA implosions while the color shows the measured YOC.

Figure 3-17 shows the ratio of the measured to predicted areal density ( $\rho r / \rho r(1 D))$ as a function of target adiabat.

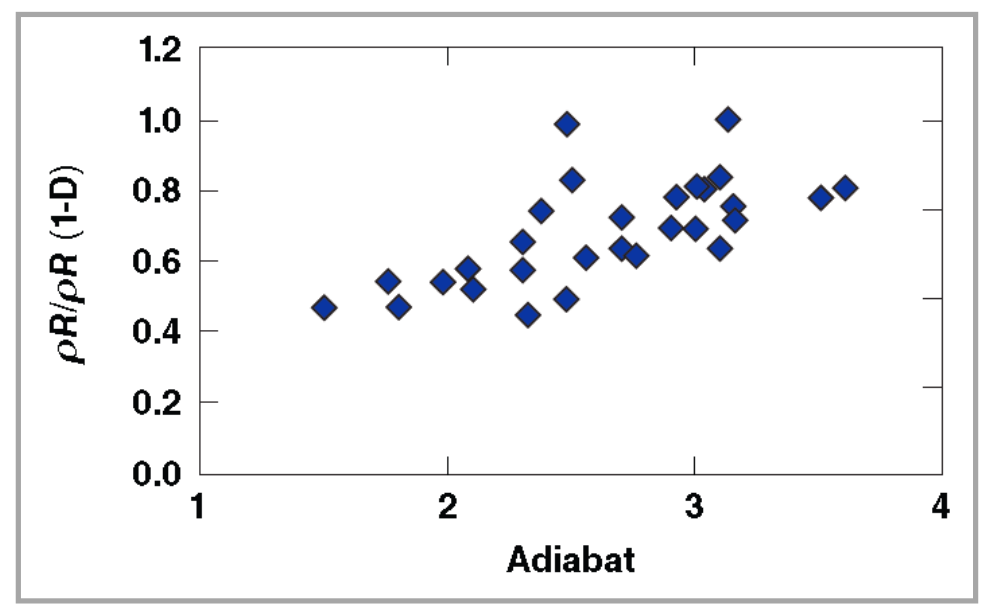

Figure 3-17. Ratio of experimental to calculated areal density as a function of target adiabat for OMEGA cryogenic target implosions.

The results show the expected trends with better performance at higher adiabats and lower IFARs. The highest-performing targets had an ion temperature of $3 \mathrm{keV}$ and neutron yields of up to $1.7 \times 10^{13}$. The areal densities are in the range of $150-300 \mathrm{mg} / \mathrm{cm}^{2}$.

Current target performance is limited by the accumulation of target surface defects during the cryogenic target fills [65]. These appear to be gases entrained in the high-pressure fill that condense on the target surface as it is cooled to liquid DT temperatures. Improved target performance has been observed with targets that have significantly fewer than the usual number of defects [65]. The targets limit the highest performing targets to adiabats above $\sim 2.5$ and IFARs less than $\sim 20$. Significant effort is being devoted to reducing the number of surface defects to improve target performance, especially at values of IFAR approaching those required for ignition (IFAR $\geq 25$ ). 


\section{National Ignition Campaign Program Completion Report}

As the number of surface defects is reduced, it is expected that laser imprinting will become the dominant determinant of target performance. Recent experiments have shown that doping the outer part of the ablator with germanium or silicon can reduce both the imprinting level and RT growth rate [66, 67]. This reduction occurs due to the smoothing of the plasma pressure gradients and imprint reduction due to the increased distance between the critical and ablation surfaces. The concept is similar to the thin high-Z outer layers proposed by the Naval Research laboratory for imprint reduction $[68,69]$.

\section{c. Path to Polar-Drive Ignition on the NIF}

The current PD ignition point design consists of a 1.5-MJ, triple-picket laser pulse driving a $3.2 \mathrm{~mm}$ diameter plastic shell enclosing a $187 \mu \mathrm{m}$ cryogenic fusion-fuel layer [56]. Two-dimensional DRACO [70] calculations indicate a target gain of $\sim 32$ when all expected sources of non-uniformity are included. Validation of the physics in these code calculations drives a large fraction of the experimental implosions on OMEGA, including those described in the previous section. The current point design ignites with an implosion velocity of $\sim 4 \times 10^{7} \mathrm{~cm} / \mathrm{s}$. A significant effort is being devoted to developing a design that ignites with an implosion velocity of $\sim 3.7 \times 10^{7} \mathrm{~cm} / \mathrm{s}$, similar to that for the indirect-drive design [3].

Critical issues include symmetric cryogenic target performance, room-temperature PD implosions detailing the drive symmetry of various pointing and defocusing schemes, as well as a series of laserplasma instability experiments investigating the effects of preheat caused by the two-plasmon-decay instability $[71,72]$ and cross-beam energy transfer between the incoming and outgoing OMEGA laser beams [63].

PD ignition designs rely on repointing beams to the equator, using different pulse shapes for different rings of the NIF configuration, and using specialized phase plates (particularly for the equatorial beams). Accurate modeling of oblique beam-energy deposition, the effect of beam obliquity on laser-plasma instabilities in the under-dense corona, and heat transport to the ablation surface are critical to achieving the adequate symmetry, implosion velocity, and shell adiabat. LLE is procuring a set of distributed phase plates that will be optimized for PD cryogenic implosions on OMEGA [57]. The targets will be smaller than the symmetric cryogenic ones to allow relevant implosion velocities and laser intensities to be used.

High-convergence PD OMEGA implosions and cone-in-shell geometries are being used to validate models of laser deposition, heat conduction, and non-uniformity growth in the ignition designs. Using beams judiciously repointed toward the equator, control of the $\ell=2$ mode has been experimentally demonstrated through backlit images of the implosion. Experiments are investigating the use of fuel/ablator layer shimming near the target equator. This reduces the laser intensity required to drive this region of the target and allows for more efficient targets to be deployed on the NIF.

PD target implosions using DT fuel have been designed and fielded for neutron diagnostic development on the NIF. The experiments use thin, room-temperature glass shells filled with low pressures (10 atm) of DT. Initial target implosions on the NIF have produced DT yields in the range of $10^{12}$ to $10^{15}$ neutrons. LLE, in collaboration with LLNL, LANL, and GA, drafted a PD Ignition Plan that provides a detailed outline of the requirements, capital resources, and timetable leading to PD ignition experiments on the NIF. This plan describes a proposed series of experiments on the NIF that would address key physics issues that are unique to the NIF high-energy environment. Such experiments initially would make use of existing NIF hardware, but then would transition to more optimally designed components as they became available. LLE has a path forward to develop PD ignition that includes ongoing OMEGA implosions (including PD) supplemented by theoretical advances and implosions on the NIF.

\section{References}

1. E.I. Moses, "Ignition on the National Ignition Facility," J. Phys. Conf. Ser. 112, 012003 (2008).

2. G.H. Miller, E.I. Moses, and C.R. Wuest, Nucl. Fusion 44, 228 (2004).

3. S.W. Haan et al., "Point design targets, specifications, and requirements for the 2010 ignition campaign on the National Ignition Facility," Phys. Plasmas 18, 051001 (2011). 


\section{National Ignition Campaign Program Completion Report}

4. W. K. Levedahl and J.D. Lindl, "Energy scaling of inertial confinement fusion targets for ignition and high gain," Nucl. Fusion 37, 165 (1997).

5. M.M. Basko and J. Johner, "Ignition energy scaling of inertial confinement fusion targets," Nucl. Fusion 38, 1779 (1998).

6. M.C. Herrmann, M. Tabak, and J.D. Lindl, "A generalized scaling law for the ignition energy of inertial confinement fusion capsules," Nucl. Fusion 41, 99 (2001).

7. R. Kishony and D. Shvarts, "Ignition condition and gain prediction for perturbed inertial confinement fusion targets," Phys. Plasmas 8, 4925 (2001).

8. D.S. Clark, S.W. Haan, and J.D. Salmonson, "Robustness studies of ignition targets for the National Ignition Facility in two dimensions," Phys. Plasmas 15, 056305 (2008).

9. O.L. Landen et al., "Capsule implosion optimization during the indirect-drive National Ignition Campaign," Phys. Plasmas 18, 051002 (2011).

10. M.J. Edwards et al., "The experimental plan for cryogenic layered target implosions on the National Ignition Facility-The inertial confinement approach to fusion," Phys. Plasmas 18, 051003 (2011).

11. C.D. Zhou and R. Betti, "A measurable Lawson criterion and hydro-equivalent curves for inertial confinement fusion," Phys. Plasmas 15, 102707 (2008).

12. P.Y. Chang et al, "Generalized Measurable Ignition Criterion for Inertial Confinement Fusion," Phys. Rev. Lett. 104, 135002 (2010).

13. J.H. Nuckolls et al., "Laser compression of matter to super-high densities: Thermonuclear (CTR) applications," Nature 239, 139 (1972).

14. S.E. Koonin, Chair, Committee for the Review of the Department of Energy's Inertial Confinement Fusion Program, Review of the Department of Energy's Inertial Confinement Fusion Program: The National Ignition Facility (National Academy Press, Washington, D.C. (1997).

15. Information on the ASC computers is available at http://www.sandia.gov/NNSA/ASC/.

16. G.B. Zimmerman and W. L. Kruer, Comments Plasma Phys. Controlled Fusion 2, 51 (1975).

17. M.M. Marinak et al., "Three-dimensional simulations of Nova high growth factor capsule implosion experiments," Phys. Plasmas 3, 2070 (1996).

18. C.H. Still et al., "Filamentation and forward Brillouin scatter of entire smoothed and aberrated laser beams," Phys. Plasmas 7, 2023 (2000); D.E. Hinkel et al., "Analyses of laser-plasma interactions in National Ignition Facility ignition targets," Phys. Plasmas 15, 056314 (2008).

19. A.A. Hauer et al., Phys. Plasmas 2, 672 (1995).

20. T.J. Murphy et al., "Hohlraum symmetry experiments with multiple beam cones on the OMEGA laser facility," Phys. Rev. Lett. 81, 108 (1998).

21. R.E. Turner et al., "Demonstration of time-dependent symmetry control in hohlraums by drivebeam staggering," Phys. Plasmas 7, 333 (2000); R.E. Turner et al., "Role of laser beam geometry in improving implosion symmetry and performance for indirect-drive inertial confinement fusion," Phys. Plasmas 10, 2429 (2003).

22. G.A. Kyrala et al., "Symmetry tuning for ignition capsules via the symcap technique," Phys. Plasmas 18, 056307 (2011).

23. E.L. Dewald et al., "Experimental demonstration of early time, hohlraum radiation symmetry tuning for indirect drive ignition experiments," Phys. Plasmas 18, 092703 (2011).

24. R.M. Malone et al., "Fielding of an imaging VISAR diagnostic at the National Ignition Facility (NIF),"Proc. of SPIE 5523, 148 (2004).

25. D.H. Munro et al., "Shock timing technique for the National Ignition Facility," Phys. Plasmas 8 , 2245 (2001).

26. T.R. Boehly et al., "Properties of fluid deuterium under double-shock compression to several Mbar," Phys. Plasmas 11, L49 (2004).

27. H.F. Robey, "An assessment of the 3D geometric surrogacy of shock timing diagnostic techniques for tuning experiments on the NIF," J. Phys.: Conf. Ser. 112, 022078 (2008). 


\section{National Ignition Campaign Program Completion Report}

28. D.G. Hicks et al., "Streaked radiography measurements of convergent ablator performance (invited)," Rev. Sci. Instrum. 81, 10E304 (2010); D.G. Hicks et al., "Convergent ablator performance measurements," Phys. Plasmas 17, 102703 (2010).

29. B. Spears et al., "Influence and measurement of mass ablation in ICF implosions," J. Phys.: Conf. Ser. 112, 022003 (2008).

30. R.E. Olson et al., "Design of a streaked radiography instrument for ICF ablator tuning measurements," Rev. Sci. Instrum. 79, 10E913 (2008).

31. D. Hicks et. al., submitted to Phys. Plasmas.

32. D.H. Kalantar et al., "X-ray backlit imaging measurement of in-flight pusher density for an indirect drive capsule implosion," Rev. Sci. Instrum. 68, 814 (1997).

33. F.J. Marshall et al., "Plasma-Density Determination from X-Ray Radiography of Laser-Driven Spherical Implosions," Phys. Rev. Lett. 102, 185004 (2009).

34. C. Cherfils et al., "Convergent Rayleigh-Taylor experiments on the Nova laser," Phys. Rev. Lett. 83, 5507 (1999); S.G. Glendinning et al., "Ablation front Rayleigh-Taylor growth experiments in spherically convergent geometry," Phys. Plasmas 7, 2033 (2000).

35. V.A. Smalyuk et al., "Rayleigh-Taylor Growth Measurements in the Acceleration Phase of Spherical Implosions on OMEGA," Phys. Rev. Lett. 103, 105001 (2009); V.A. Smalyuk et al., "Spherical Rayleigh-Taylor growth of three-dimensional broadband perturbations on OMEGA," Phys. Plasmas 16, 112701 (2009).

36. D.K. Bradley et al., "D-T gamma-to-neutron branching ratio determined from inertial confinement fusion plasmas," Phys. Plasmas 14, 056313 (2007).

37. O.L. Landen et al., J. Phys.: Conf. Ser. 112, 022004 (2008).

38. B.A. Hammel et al., "Diagnosing and controlling mix in National Ignition Facility implosion experiments," Phys. Plasmas 18, 056310 (2011); S.P. Regan et al., "Hot-spot mix in ignitionscale implosions on the NIF," Phys. Plasmas 19, 056307 (2012).

39. J.M. Gostic et al., "Solid debris collection for radiochemical diagnostics at the National Ignition Facility," Rev. Sci. Instrum. 83, 10D904 (2012).

40. R. Tommasini et al., Phys. Plasmas 18 (2011) 056309

41. S.H. Glenzer et al., "Cryogenic thermonuclear fuel implosions on the National Ignition Facility," Phys Plasmas 19, 056318 (2012).

42. S.P. Regan et al., "Hot-spot mix in ignition-scale implosions on the NIF," Phys. Plasmas 19, 056307 (2012).

43. R. Betti et al., "Growth rates of the ablative Rayleigh-Taylor instability in inertial confinement fusion," Phys. Plasmas 5, 1446 (1998).

44. O.S. Jones et al., "A high-resolution integrated model of the National Ignition Campaign cryogenic layered experiments," Phys. Plasmas 19, 056315 (2012)

45. E.N. Loomis, "Areal density evolution of isolated surface perturbations at the onset of x-ray ablation Richtmyer-Meshkov growth," Phys. Plasmas 18, 092702 (2011).

46. J.P. Knauer et al., "Improved target stability using picket pulses to increase and shape the ablator adiabat," Phys. Plasmas 12056306 (2005).

47. A.J. Mackinnon et al., "Assembly of High-Areal-Density Deuterium-Tritium Fuel from Indirectly Driven Cryogenic Implosions," Phys. Rev Lett. 108215005 (2012).

48. S. Skupsky et al., "Polar Direct Drive on the National Ignition Facility," Phys. Plasmas. 11(5), 2763 (2004).

49. V.N. Goncharov et al., "Demonstration of the Highest Deuterium-Tritium Areal Density Using Multiple-Picket Cryogenic Designs on OMEGA," Phys. Rev. Lett. 104(16), 165001 (2010).

50. T.C. Sangster et al., "Shock-Tuned Cryogenic-Deuterium-Tritium Implosion Performance on OMEGA," Phys. Plasmas 17(5), 056312 (2010).

51. J. Delettrez, "Thermal Electron Transport in Direct-Drive Laser Fusion," Can. J. Phys. 64(8), 932 (1986).

52. T.R. Boehly et al., "Initial Performance Results of the OMEGA Laser System," Opt. Commun. 133, 495 (1997). 


\section{National Ignition Campaign Program Completion Report}

53. L.J. Waxer et al., "High-Energy Petawatt Capability for the OMEGA Laser," Opt. Photonics News 16(7), 30 (2005).

54. A.M. Cok, R.S. Craxton, and P.W. McKenty, "Polar-Drive Designs for Optimizing Neutron Yields on the National Ignition Facility," Phys. Plasmas 15(8), 082705 (2008).

55. V.N. Goncharov et al., "Multiple-Picket Cryogenic Designs for OMEGA and the NIF," to be published in Physical Review Letters (2009).

56. T.J.B. Collins et al., "A Polar-Drive-Ignition Design for the National Ignition Facility," Phys. Plasmas 19(5), 056308 (2012).

57. P.B. Radha et al., "OMEGA Polar-Drive Target Designs," Phys. Plasmas 19(8), 082704 (2012).

58. M.C. Herrmann, M. Tabak, and J.D. Lindl, "A Generalized Scaling Law for the Ignition Energy of Inertial Confinement Fusion Capsules," Nucl. Fusion 41(1): p. 99-111 (2001).

59. C.D. Zhou and R. Betti, "Hydrodynamic Relations for Direct-Drive Fast-Ignition and Conventional Inertial Confinement Fusion Implosions," Phys. Plasmas 14(7), 072703 (2007).

60. R. Betti et al., "Theory of Laser-Induced Adiabat Shaping in Inertial Fusion Implosions: The Relaxation Method," Phys. Plasmas 12, 042703 (2005).

61. V.N. Goncharov, "Direct-Drive Inertial Fusion: Basic Concepts and Ignition Target Designing," Laser-Plasma Interactions, Boca Raton, FL: CRC Press (2009).

62. P. Michel et al., "Energy Transfer Between Laser Beams Crossing in Ignition Hohlraums," Phys. Plasmas 16(4), 042702 (2009).

63. I.V. Igumenshchev et al., "Crossed-Beam Energy Transfer in Direct-Drive Implosions," Phys. Plasmas 19(5), 056314 (2012).

64. W.L. Kruer et al., "Energy Transfer Between Crossing Laser Beams," Phys. Plasmas 3(1), 382 (1996).

65. T.C. Sangster et al., "Cryogenic-DT-Implosion Performance with Improved Target-Surface Quality," Bull. Am. Phys. Soc. 56(12), 241 (2011).

66. S.X. Hu et al., "Mitigating Laser Imprint in Direct-Drive Inertial Confinement Fusion Implosions with High-Z Dopants," Phys. Rev. Lett. 108(19), 195003 (2002).

67. G. Fiksel et al., "Experimental Reduction of Laser Imprinting and Rayleigh-Taylor Growth in Spherically Compressed, Medium-Z-Doped Plastic Targets," Phys. Plasmas 19(6), 062704 (2012).

68. A.N. Mostovych et al., "Enhanced Direct-Drive Implosion with Thin High-Z Ablation Layers," Phys. Rev. Lett. 100(7), 075002 (2008).

69. S.P. Obenschain et al., "Effects of Thin High-Z-Layers on the Hydrodynamics of LaserAccelerated Plastic Targets," Phys. Plasmas 9(5), 2234 (2002).

70. P.B. Radha et al., "Multidimensional Analysis of Direct-Drive, Plastic-Shell Implosions on OMEGA," Phys. Plasmas 12, 056307 (2005).

71. D.T. Michel et al., "Experimental Demonstration of the Two-Plasmon-Decay Common-Wave Process," to be published in Physical Review Letters 2012.

72. W.L. Kruer, "The Physics of Laser Plasma Interactions," Frontiers in Physics, Boulder, CO: Westview Press, p. 39 (2003). 


\section{National Ignition Campaign Program Completion Report}

\section{I.4-INTEGRATED TARGET SYSTEMS}

\section{I.4.1-Target Development and Manufacturing}

The main scope of work conducted under the NIC WBS Element I.4.1, Target Development and Manufacturing, is establishing the capability to develop, design for manufacturability, and fabricate capsules, hohlraums, and all the diagnostic and alignment components for ignition targets and targets required for supporting experiments. This effort also encompasses the equipment needed to manufacture, assemble, and characterize the target components and assemblies.

\section{A. Ignition Target Design Development}

NIC requires complex and precise targets to perform its mission. The most complicated of these are the indirect-drive cryogenic ignition targets. At the center of these targets is a $~ 2$ $\mathrm{mm}$ diameter capsule that is filled with hydrogen fuel - in most cases, a solid layer of a 50:50 equimolar mixture of deuterium and tritium in equilibrium with its vapor at $1.3-1.5$ $\mathrm{K}$ below the triple point at shot time. The capsule and the fuel are concentric and centered inside a high-Z hohlraum. An example of an ignition target is shown in Figure 4-1.

A concentrated technology development program, with particular emphasis on precision engineering design with respect to applied materials research and development, has enabled the Target Manufacturing team to

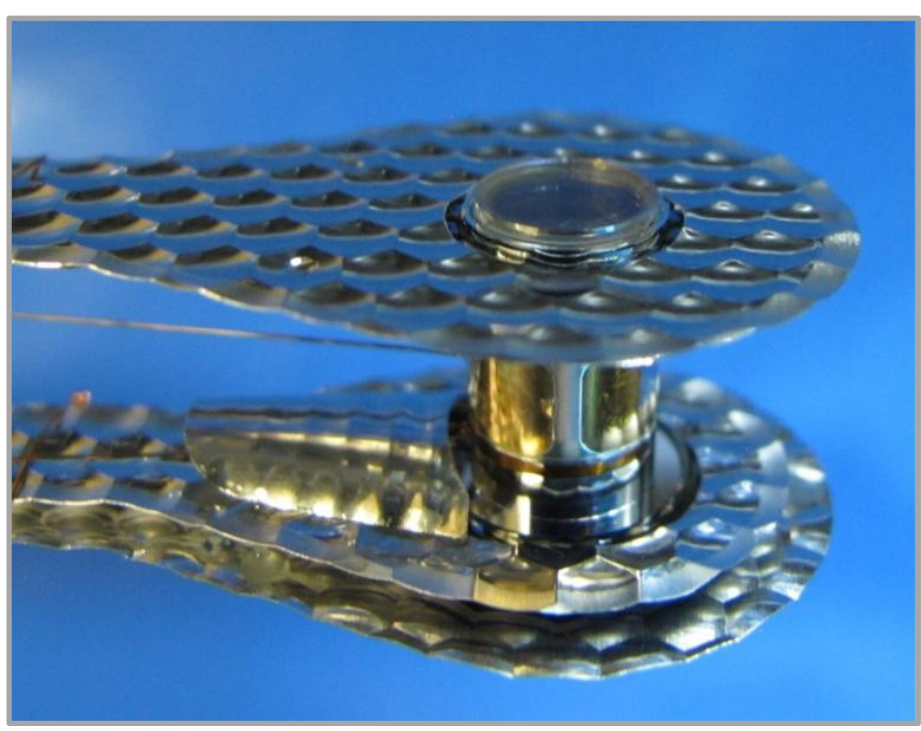

Figure 4-1. A layered cryogenic ignition target. meet the stringent NIC requirements for capsule surface finish and positioning, performance at cryogenic temperatures, and robustness during assembly and fielding. Each target component was analyzed, and new designs and processes were developed, verified, tested, implemented, and documented. Assembly and tooling stations and procedures were created to meet target positioning requirements, and component design changes were implemented to improve assembly yield and throughput. Because requirements change due to experimental feedback, the target technology research and development must be continuous to keep pace. The Target Manufacturing team has excelled in providing precision targets for the NIC, showing a unique ability to respond dynamically as new targets are required.

The NIC ignition target consists of an ablator capsule and fill tube, hohlraum, LEH inserts, tents, and the Thermal Mechanical Package (TMP). A TMP consists of silicon cooling arm, thermal shell, diagnostic band, windows, heaters and sensors, tamping gas line, and wiring harness (see Figure 4-2). 


\section{National Ignition Campaign Program Completion Report}

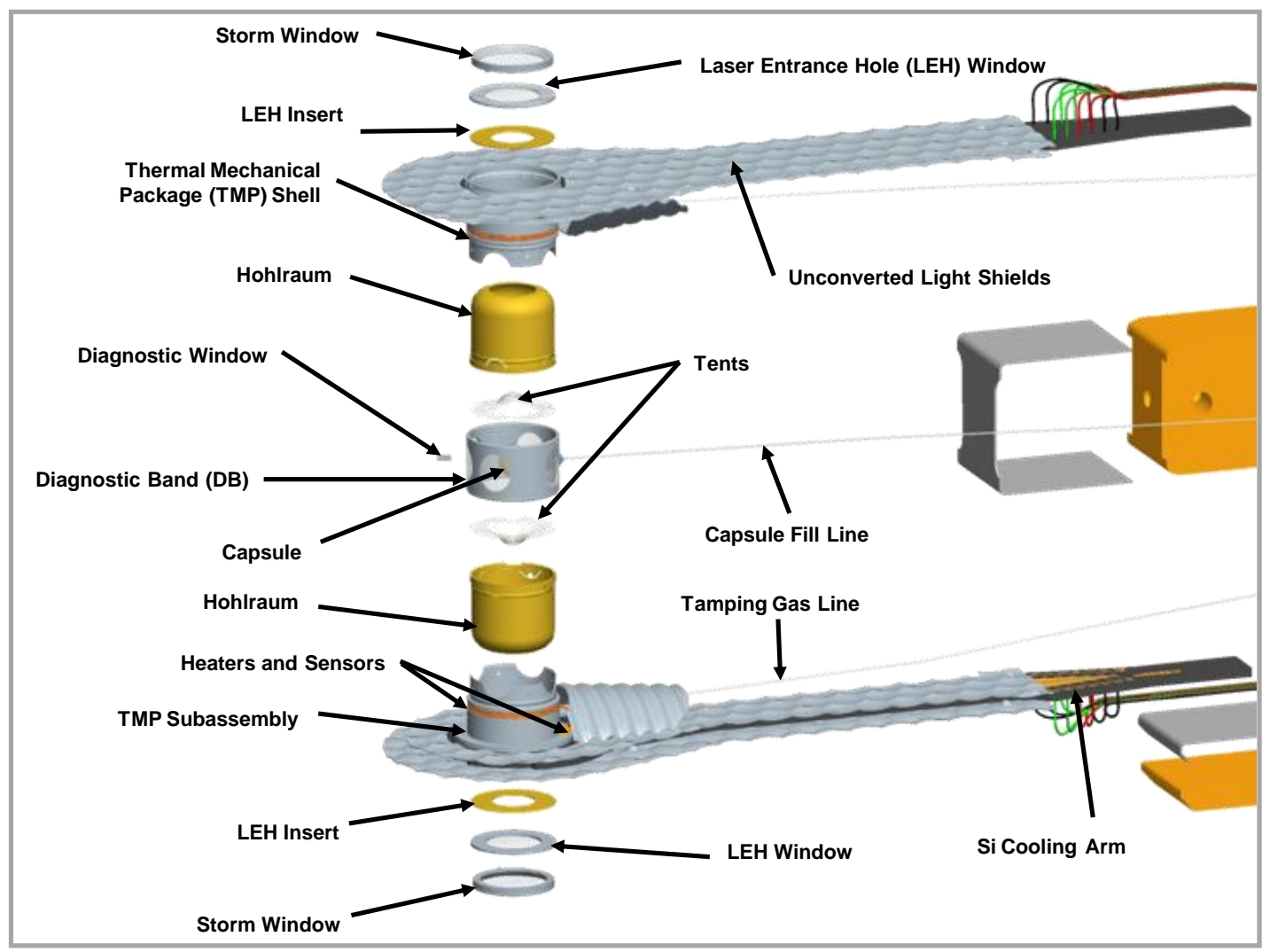

Figure 4-2. Exploded view of an ignition target.

Production of the capsule starts with a very smooth, very spherical plastic mandrel, the form on which the silicon-doped plastic is deposited uniformly as the mandrel is rolled or rotated. The mandrel material is chosen so that it depolymerizes into its monomer form at $300^{\circ} \mathrm{C}$. The mandrel material is thus removed by heating, leaving the stable silicon-doped coating behind as the final shell. The silicon-doped shells are then characterized to ensure that they meet dimensional and dopant content requirements and are polished as appropriate to improve surface finish. A fill hole, typically ten microns in diameter, is then laser drilled into the capsule. A precisely drawn and finished glass fill tube is attached to the capsule for DT gas delivery $[1,2]$.

The ignition hohlraum is a gold or goldcoated (to prevent oxidation) uranium cylinder designed to couple as much laser energy to the capsule as possible. The layers are sputter-deposited on a precision-machined mandrel, which is etched away after deposition and machining are complete. The hohlraum is equipped with dual tori on the outer diameter for precision positioning within the thermal aluminum shell. A flange at the waist of the hohlraum supplies a stop for the insertion in the TMP and an

attachment surface for the thin Formvar tent that positions the capsule. The LEH

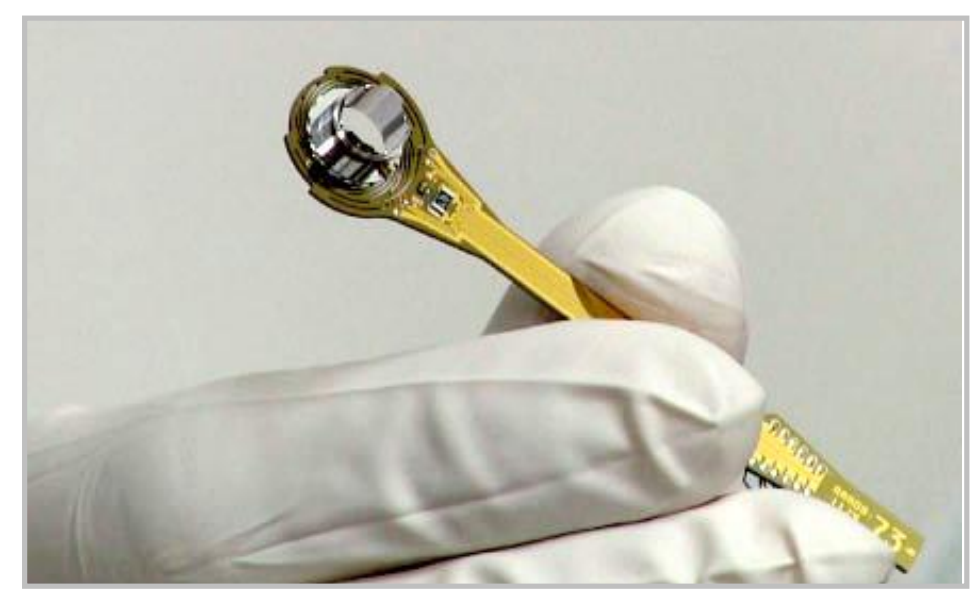

Figure 4-3. TMP subassembly. inserts are machined as separate parts to 


\section{National Ignition Campaign Program Completion Report}

allow for various LEH diameters, and the LEH windows are aluminum coated, 500 nanometer thick polyimide film [3]. The diagnostic band joins and aligns the subassembled target halves and provides ports for characterization and diagnostic access.

The TMP (see Figure 4-3) precisely positions the hohlraum, manages the thermal environment of the hohlraum and capsule, and provides a modular platform for various diagnostic configurations without changes to the remaining components. The TMP consists of two aluminum shells joined by a band with cutouts to accommodate various diagnostics requirements. The design is modular and has been used throughout the ignition campaign.

The two silicon cooling arms attached to either end of the TMP assembly conduct heat away from the hohlraum to maintain the required temperature. These are lithographically etched to create a precise heat transfer path that ensures temperature uniformity in the target. Heaters located on the TMP shells are then used to produce a nearly spherical isotherm around the capsule. Tamping gas lines deliver helium gas to the hohlraum to mitigate the interaction between laser light entering the hohlraum and the ablated material from the hohlraum walls, as this interaction can steer and scatter the light.

It is important to note that this generic overview of the target understates the complexity of target manufacturing. A target often consists of up to 500 individual precision-manufactured components, as specified by the particular target physics campaign. More detail on the ignition target design can be found in a paper by Alger et al. [4]

\section{B. NIC Surrogate Physics Targets}

A fundamental set of surrogate physics targets, fielded at cryogenic temperatures, are used as part of NIC (see Figure 4-4). These surrogate targets are not intended to produce ignition; instead, they generate essential physics information on target conditions to help refine ignition experiment parameters.

As discussed in Section I.3(d), Indirect-Drive Implosion Optimization, these include the Re-emit target, used to tune early-time radiation symmetry in the hohlraum; the Keyhole target, used to establish shock timing and power levels of the ignition laser pulse; the Convergent Ablator (ConA) target, used to measure the capsule trajectory ${ }^{\mathrm{q}}$ and determine mass remaining at bang time ${ }^{\mathrm{r}}$; and the Symcap, used to adjust implosion symmetry. The Symcap is essentially an ignition target but with a thicker plastic capsule layer replacing the DT fuel. In an iterative process that has continued throughout the campaign, the implosion parameters are refined using these targets, and integrated experiments using an ignition target complete with a cryogenic fuel layers are then conducted to assess implosion performance.

${ }^{\mathrm{q}}$ Capsule trajectory is the change in capsule radius as a function of time during an implosion.

${ }^{\mathrm{r}}$ Bang time is the time interval between the start of the laser pulse and peak $\mathrm{x}$-ray emission from the fuel core in inertial confinement fusion experiments.

${ }^{\mathrm{s}}$ Ignition experiments use equimolar (50:50) deuterium and tritium (DT) fuel; however, THD experiments contain a tritium, deuterium, and hydrogen mix. THD experiments are designed to produce neutron yields low enough to use a full suite of x-ray and neutron diagnostics that would not be available for the higher neutron production of DT implosions. 


\section{National Ignition Campaign Program Completion Report}

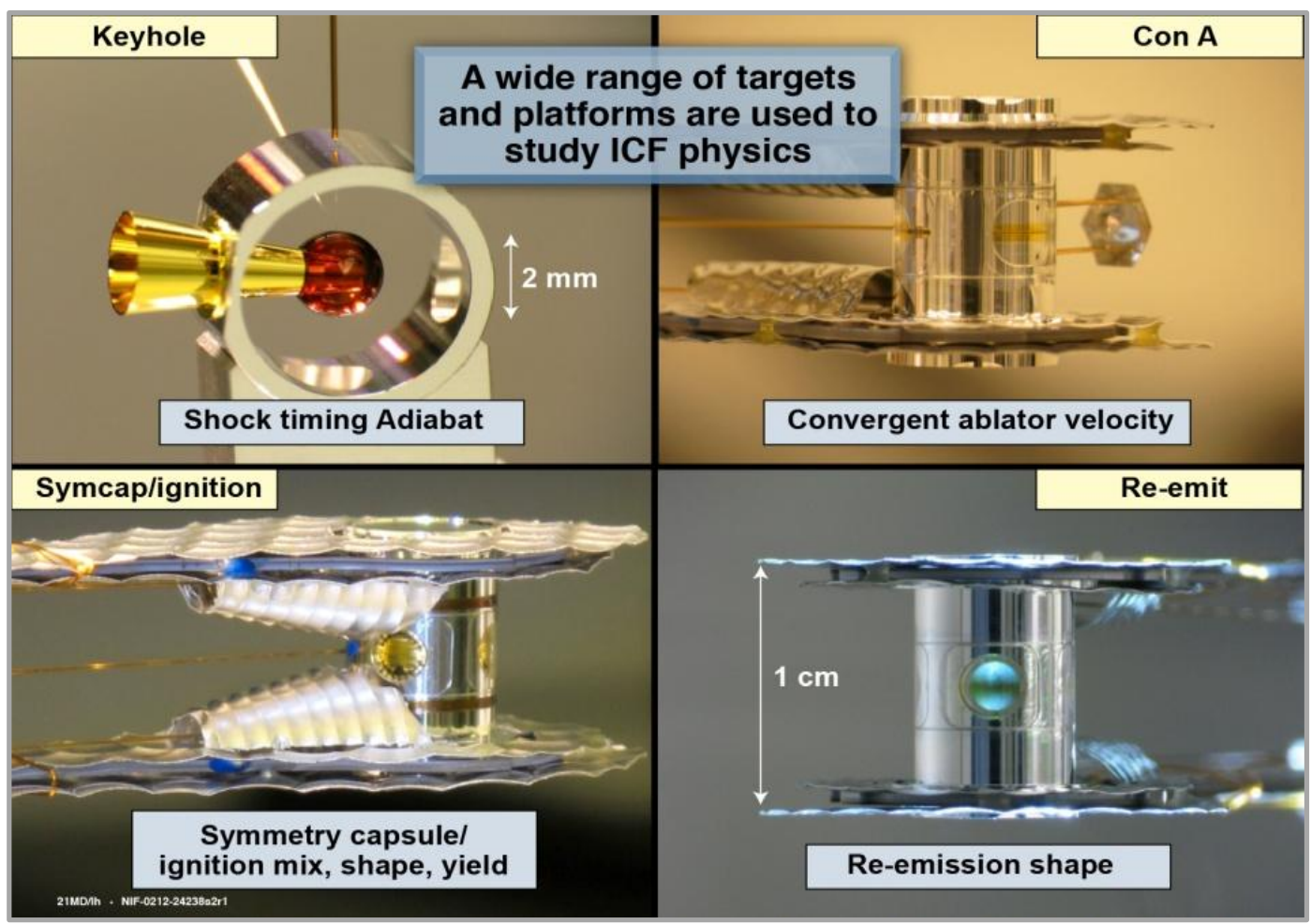

Figure 4-4. Surrogate physics targets used in NIC.

\section{Major Target Modifications and New Target Designs}

Often modifications to existing target designs or new target designs are required to respond to the evolving needs of the ignition experimental program. For example, to measure the implosion trajectory over a longer time period, the viewing slit in the hohlraum for ConA targets had to be increased significantly. The diagnostic axis and thus the location of the backlighter for this target were also changed to allow relocation of the primary diagnostic. This resulted in a significant reduction in diagnostic reconfiguration time, hence increasing the shot rate on NIF. The Keyhole target is another case where a major design modification was required. To resolve a physics issue relating to possible shock asymmetry in Keyhole shock timing experiments, the Keyhole target was redesigned to incorporate a small internal mirror to obtain shock transit information using the Velocity Interferometer System for Any Reflection (VISAR) diagnostic from both the equatorial and polar directions of the capsule simultaneously. This modified target is called the dual-axis Keyhole target and is shown in Figure 4-5.

During NIC, the ignition targets were modified to improve layering and reduce radiation losses through diagnostic openings by adding a heater to remove non-concentricity observed in solid fuel layers in depleted uranium hohlraums and gold coating the diamond window. The TMP was also redesigned to minimize the impact of future target scale changes and allow quicker response to changes in hohlraum size (length and diameter), shape (rugby), and case-to-capsule ratio (ratio of hohlraum to capsule dimensions). Ignition targets were also made impervious to frost formation by adding a second, warmer

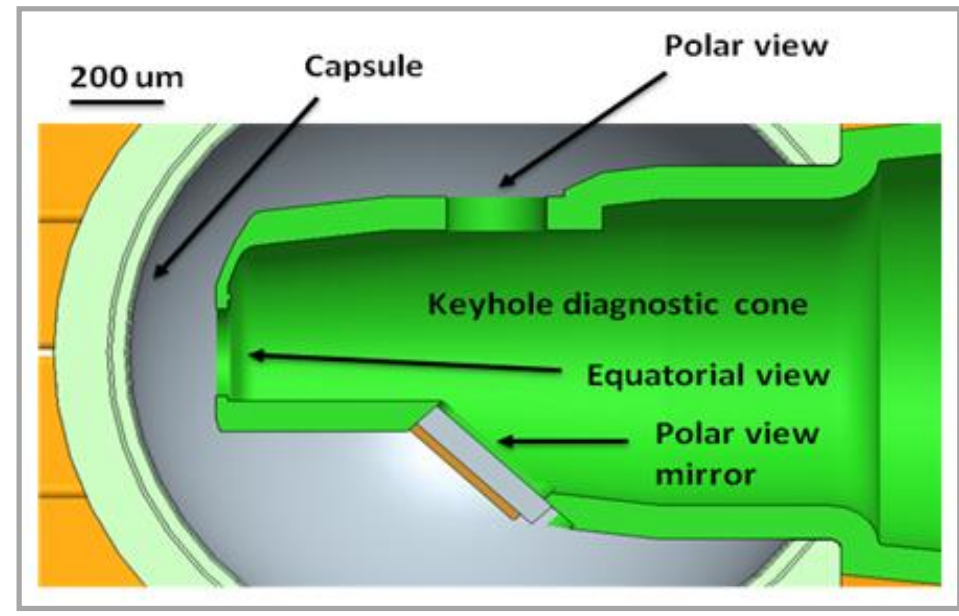

Figure 4-5. The Keyhole target was redesigned to incorporate a small mirror to support VISAR diagnostic data gathering. 


\section{National Ignition Campaign Program Completion Report}

window, often called a "storm window," on the top of each LEH.

In addition, the Target Manufacturing team responded to requests for completely new targets designs, all of which had to be designed and fabricated to meet the demanding experimental schedule. Examples of these included the CR target, which uses a backlighter to image the cold fuel during the implosion; the Crystal Ball target, which diagnoses hohlraum drive pressure and timing by measuring the shock timing in materials where the equations of state are well known; and the View Factor target, which measures the radiation flux and temperature in a hohlraum at the capsule equator. See Figure 4-6.

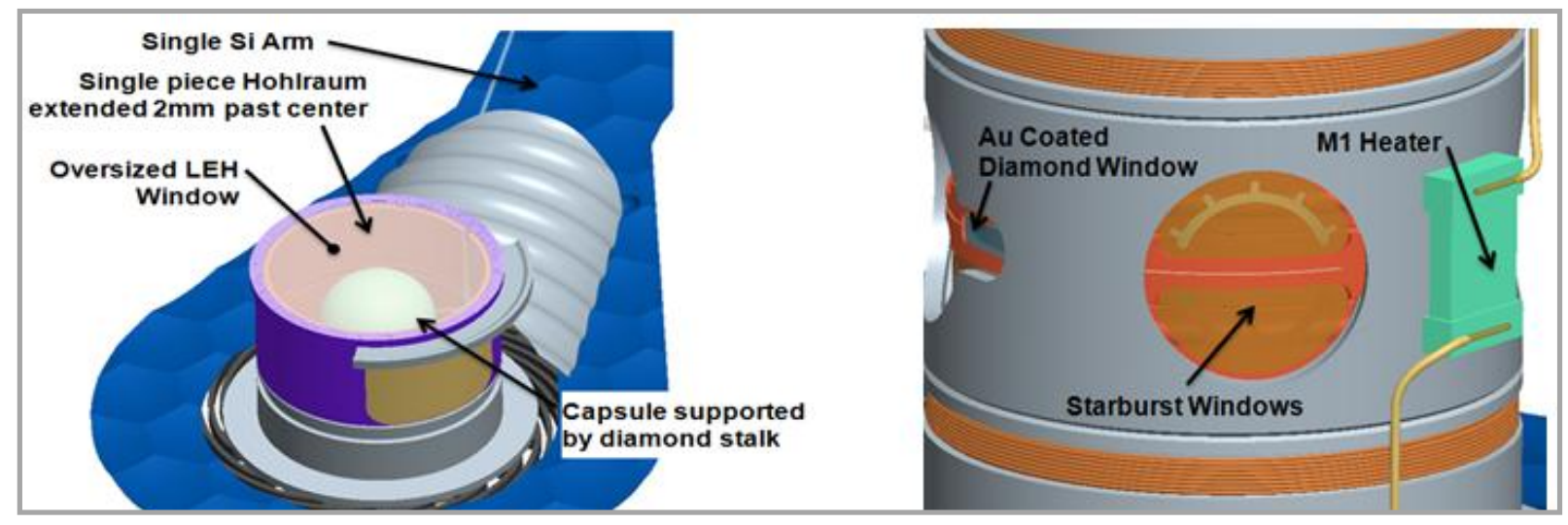

Figure 4-6. View Factor and tritium-hydrogen-deuterium target upgrades.

\section{Continuous Improvements in Target Quality and Target Assembly}

The extreme temperatures and pressures the targets encounter during experiments make the results susceptible to imperfections in fabrication. The specific manufacturing requirements for all NIF targets are extremely precise. Components have to be machined to an accuracy of within one micrometer, or one millionth of a meter. Some joints can be no larger than 100 nanometers. Precise microassembly of the targets has an error margin of less than three microns. New tools to image and characterize materials have provided insight into the target development progress and necessary changes in material preparation and fabrication. Continual improvements are also based on simulations and experiments at the Janus laser at LLNL, the OMEGA laser at the University of Rochester, and other facilities worldwide.

\section{Capsule Finishing}

Surface conditions for the assembled fuel capsules have stringent specifications, and most capsules require polishing to meet these specifications. In the case of plastic ablators, the major polishing concern is controlling isolated defects on the surface. The defects arise from submicron features in all dimensions on the mandrel; their size is such that optical detection is very limited. They evolve into 30-40 micron diameter bumps 200-800 nanometers in height. Conventional polishing can remove the bumps but leaves scratches behind. A proprietary polishing technique has been developed that can remove or reduce bumps from 600 nanometers to less than 150 nanometers in height with minimal scratching. Larger bumps require a laser ablation step before polishing to reduce their height to less than the 600 nanometers.

Scanning electron micrographs show that polishing a plastic capsule improves its smoothness and consistency (see Figure 4-7). These capsule finishing techniques were developed in approximately a year. 


\section{National Ignition Campaign Program Completion Report}

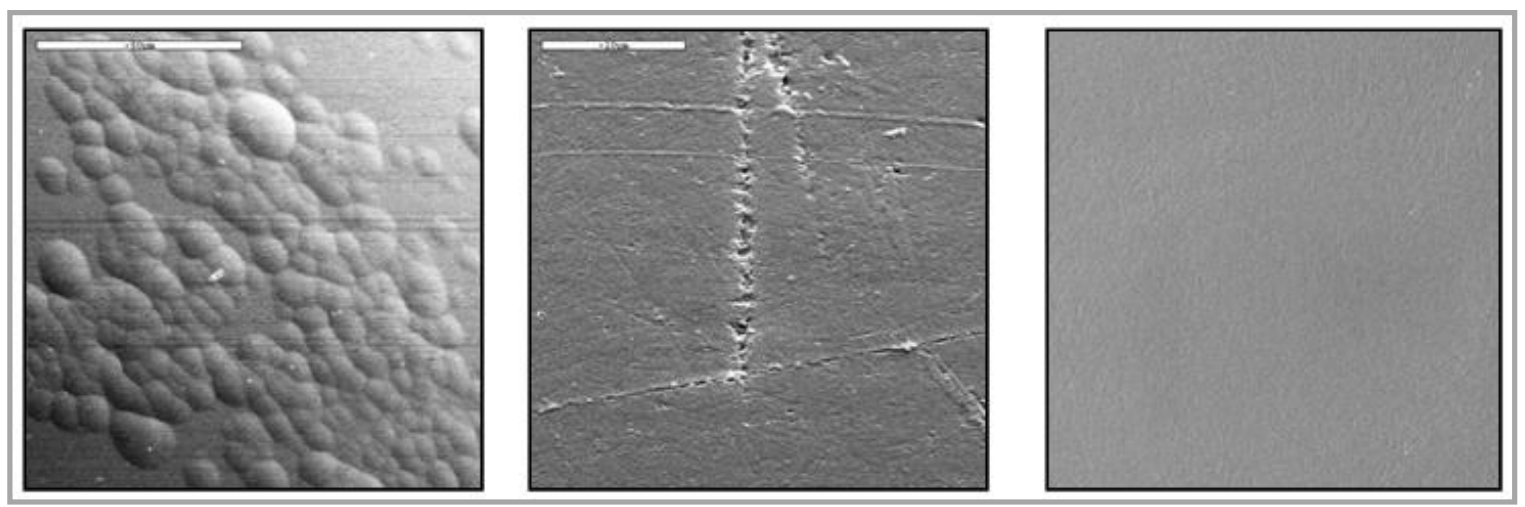

Figure 4-7. Scanning electron micrographs of capsule surface. Left to right: as-deposited, conventional polish, advanced finishing.

\section{New Materials and Processes Developed}

As the NIC experimental campaign has progressed, target physicists and target manufacturing engineers have collaborated to develop new materials and processes to enhance target performance and diagnosis and/or make target production more efficient. Examples of hohlraum design and manufacturing developments include:

- The use of micron-layered material coatings (of depleted uranium, for example).

- Precision doping of capsule ablators (by adding silicon to the capsule wall, for example) to enable the detection of specific x-ray signatures during a shot.

- The addition of trace amounts of entrapped gases to the capsule (such as xenon) [5] for after-shot radioisotope forensics [6].

- New ablator materials (such as high-density carbon) for improve x-ray energy coupling [7].

- Modifications to the tent that positions the capsule inside the hohlraum (to reduce the amount of material and adjust the capsule wall characteristics during ablation) [8].

- Reduction of the fill tube diameter (from an already miniscule 10 micron diameter to less than 5 microns) to minimize its effect on capsule implosion symmetry.

Of particular note is the effort to reduce the fill tube diameter. The fill tube delivers fuel to the target and is thought to have a role in the initiation of the crystal growth of the ice layer. At issue was the possibility of an implosion asymmetry introduced by the fill tube, as it penetrates the capsule wall at one point.

Moving to a smaller diameter fill tube could minimize the asymmetry effect but would require drilling a 5 micron hole through the 200-micron-thick ablator, a difficult 40:1 aspect ratio. Nevertheless, the engineering teams successfully completed the process engineering steps needed to make the fill tube area four times smaller - creating the new fill tube, drilling the desired hole, manipulating the smaller tube and gluing it in place, examining the effect of the smaller tube on capsule fill time [9], and finally putting all the processes through a rigorous scientific peer review. The result is a process that may increase the reliability of crystal seeding during fuel layering and reduce the already miniscule capsule asymmetry, an achievement accomplished while continuously producing the current capsules required by the target physicists.

\section{Target Assembly}

Several methods have been used in target assembly to position the capsule in the center of the target, each with associated downsides relative to sealing joints at cryogenic temperatures. Currently, the most robust method for capsule positioning is threading the fill tube through a 200-micron hole in the diagnostic band, roughly positioning the capsule in the center of the band. The capsule fill tube assembly (CFTA) and diagnostic band are then moved into the final assembly station as a subassembly. This method has been repeatedly shown to be helium leak tight. The main concern when assembling the CFTA to the diagnostic band is protecting the capsule-to-fill tube joint, which is made with 5 nanograms (specification) of epoxy. 


\section{National Ignition Campaign Program Completion Report}

The final assembly integrates two TMP subassemblies, the CFTA and the diagnostic band, simultaneously. It also integrates the target and the target cryogenic base.

An important consideration during final assembly is that the workstation and related characterization systems are aligned prior to and during assembly. Therefore, the final assembly machine is integrated on an Optical Coordinate Measuring Machine. Precision tooling used to bring the target halves together must keep the assemblies square to each other and properly clocked. Force and torque sensors are integrated into the final assembly machine to monitor any misalignment or binding. A critical element of the final assembly is capsule centering. The capsule is centered with respect to the hohlraum-LEH inner diameter via micrometer-driven stages, but once the tents engage the capsule, the position is controlled by the mechanical properties of the tent. Capsule centering metrology is done optically via microscopy and laser ranging.

The NIC infrastructure for making and characterizing the precision cryogenic target components resides at GA. LLNL, on the other hand, is responsible for assembly of these components and the characterization of the "as-built" cryogenic targets (ignition and surrogate physics targets) used in the NIC experimental ignition program.

Target assembly is carried out at LLNL in Building 381 in a 3000-square-foot, Class 100 cleanroom (see Figure 4-8). The cleanroom is equipped with over 40 assembly stations with customized tooling, where components arriving from GA, in-house fabrication facilities, and outside vendors are inspected, assembled, and tested to produce shot-ready, cryogenic targets for NIC.

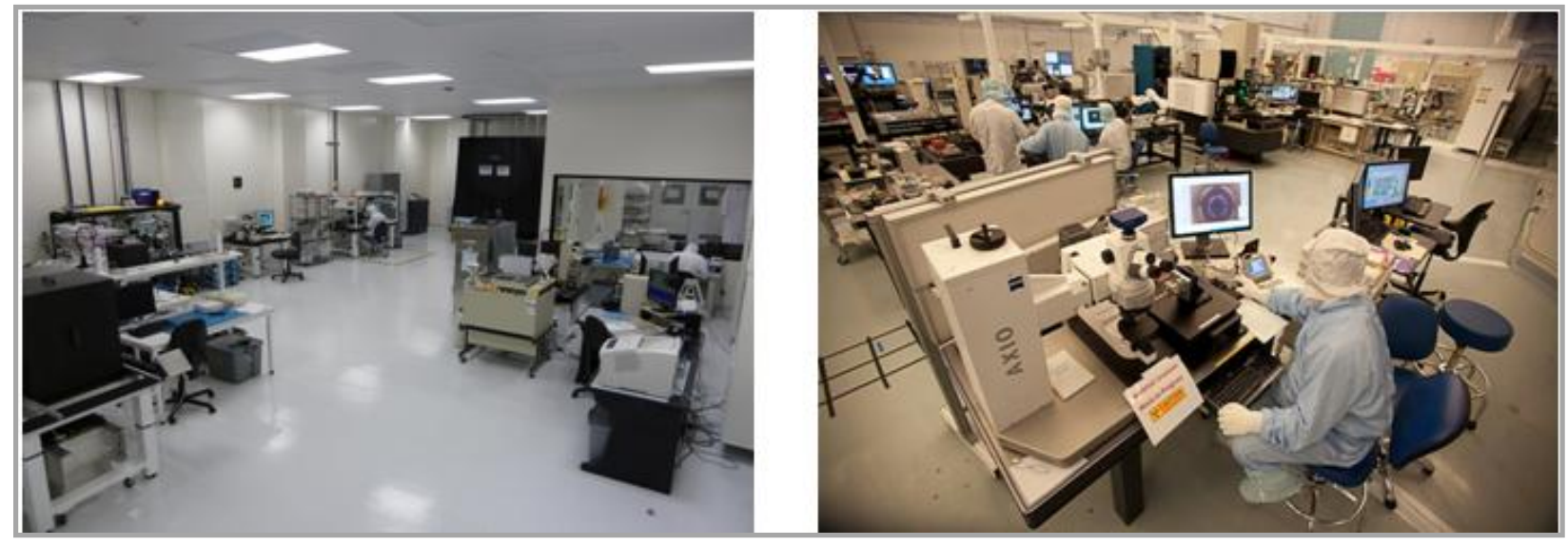

Figure 4-8. Cleanroom for fabrication and characterization of target components at General Atomics is shown on the left, while the cleanroom at LLNL for target assembly and characterization is shown on the right.

Four distinct assembly lines have been developed to assemble the intricate NIC targets to the required precision. They are:

1. Tent subassembly line — used for fabricating, installing, and inspecting the Formvar membranes that support the capsule in the center of the target.

2. CFTA subassembly line - used for confocal microscopy $4 \pi$ inspection and additional cleaning (if required) of the CFTA, and threading the CFTA into final assembly tooling.

3. TMP subassembly line - used to marry the hohlraum and LEH insert with the TMP.

4. Final assembly line - brings together the components and subassemblies from the other three lines and completes the integration of these components, including sealing the target so that it is leak tight at both room and cryogenic temperatures [10].

Fabrication of NIF ignition targets has evolved to a greater level of automation and determinism through the use of a new Precision Robotic Assembly Machine (see Figure 4-9) [11]. Error budgets supporting the fabrication and validation of critical assemblies have been formulated and validated through extensive metrology of completed targets. A suite of new assembly tools provides increased throughput with greater 


\section{National Ignition Campaign Program Completion Report}

repeatability, while offering agility in accommodating varying size scales and novel target features. A process for rapid-close target assembly was designed and implemented to assemble a CFTA into an ignition target while maintaining capsule orientation.

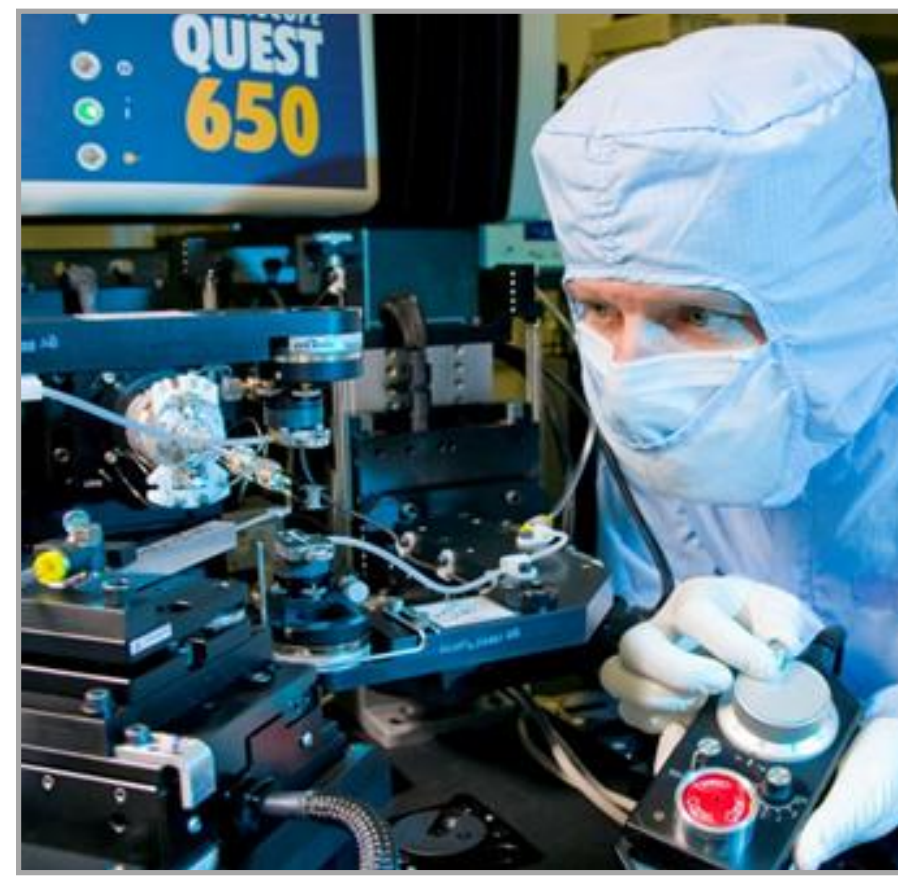

Figure 4-9. (Left) The Precision Robotic Assembly Machine used to manufacture fusion ignition targets. (Right) High-throughput capsule cleaning process uses a combination of chemical action (solvent choice) and physical entrainment (stream kinematics) to clean capsule surfaces with high efficiency.

Throughout the target assembly process, a critical element is cleanliness. Surface debris and imperfections can interfere with the uniformity of capsule heating and compression. The external and internal capsule surfaces are the smoothest surfaces fabricated during the target manufacturing process. The capsules undergo a multistep manufacturing process to ensure precision and performance reliability through interior and exterior capsule smoothness. Capsules are inspected and then subjected to a tumble polish. If during assembly any features or spots higher than 2 microns are detected, they are individually mapped (a 2 micron height variation on a 2000 micron diameter sphere represents a variation of $0.1 \%$ in height) and treated. A solvent stream jet is used to remove isolated particles adhering to the surface of the capsule. The cleaned capsule is inspected and characterized, and the capsule is then ready for hohlraum insertion.

\section{E. Target Fabrication and Manufacturing Infrastructure}

To meet the demanding precision, schedule, and volume requirements for manufacturing the wide array of targets used during the NIC, numerous capabilities have been put into place both at GA and LLNL. Further, processes have been developed and corresponding procedures have been documented to ensure high-quality component fabrication, target assembly, and characterization on a consistent basis. These have formed the basis of training for the target production workforce and for ensuring continuity and retention of expertise. The extraordinary capabilities and infrastructure developed and implemented for target fabrication and manufacturing under NIC will broadly support all NIF missions in the future.

\section{Capabilities at GA (La Jolla, California)}

The production of target components and subassemblies for NIC is carried out in numerous laboratories located in the main GA campus.

Precision Machining: A precision machining area includes seven precision lathes and three precision mills used in the fabrication of TMP components, LEH components, keyhole cones, mirrors, shells, and hohlraums. Two laser machining areas are used for drilling holes in plastic $(\mathrm{CH})$, beryllium, and highdensity carbon shells, and a laser ablation station that is used to remove isolated features on $\mathrm{CH}$ shells to reduce the effect of such defects on the implosion. 


\section{National Ignition Campaign Program Completion Report}

Mandrel Fabrication: Mandrels for $\mathrm{CH}$ capsules and beryllium shells are fabricated in a cleanroom.

Capsule Fabrication: Ten glow discharge plasma coating systems [12] are available for making capsules, five of which are dedicated to NIF CH shell fabrication. The other coating systems are used for depositing custom layers (such as copper or germanium) or silicon dopant layers used as X-ray preheat shields. Custom layering also includes the option to add small amounts of detector material at specific locations in the capsule to trace capsule material mixing into the central hot spot region. Capabilities have been introduced for polishing both $\mathrm{CH}$ and beryllium capsules. Glass exploding pusher target shells used for neutron diagnostic calibration at NIF are also fabricated and metrologized using the glow discharge polymer process.

Hohlraums: In addition to gold hohlraums, GA has facilities for fabricating depleted uranium (DU) hohlraums. A precision lathe is dedicated to machining DU and copper or aluminum mandrels used in DU hohlraum fabrication, and three coating systems are used to coat mandrels for DU hohlraum fabrication.

Subassemblies: Subassembly of the CFTA and TMP cans is performed in a cleanroom equipped with a number of customized assembly stations and specialized metrology equipment. A robotic assembly area has been qualified for production to automate the attachment of TMP cans to the silicon arms. This subassembly is a critical component of the final assembly at NIF, where the target is connected to the cryogenic base.

Beryllium: GA has a facility capable of safely handling beryllium, equipped with three beryllium sputter coating systems used in fabrication of beryllium shells [13].

Metrology: Several laboratories at GA are set up and equipped to carry out precision characterization [14] of NIC target components (TMP components, LEH components, keyhole cones, mirrors, mandrels, capsules, and hohlraums, and TMP and CFTA subassemblies). Capabilities include a Nikon Nexiv optical coordinate measuring machine equipped with custom analysis software for automated mandrel dimensional metrology.

GA fully characterizes NIC capsules using the following techniques (see Figure 4-10).

- Phase Shifting Diffractive Interferometry (PSDI): The PSDI functions by passing a single 532 $\mathrm{nm}$ laser beam through a beam splitter, which divides the beam into a reference and measurement beam. These beams are reflected to the interferometer and undergo constructive and destructive interference. Fringes are produced as the beams go in and out of phase, and a charge-coupled device (CCD) camera detects and displays the resultant interferogram. A delay in the beam is introduced by a piezoelectric transducer (PZT) that moves a total distance of approximately onehalf wavelength. A CCD image is taken each time the PZT moves $1 / 24$ of a wavelength to generate twelve $2 \mathrm{k}$ by $2 \mathrm{k}$ pixel arrays. By fitting a sine wave to the 12 arrays, the phase angle is obtained for every pixel. This phase angle is directly proportional to the surface height at each pixel, resulting in a height map. Captured CCD images have a lateral resolution of $\sim 1 \mu \mathrm{m}$ and height accuracy of $\sim 5 \mathrm{~nm}$ peak to valley. In its current embodiment, the interferometer uses 110 images (medallions) to capture all isolated and gently curved defects on the entire shell surface [15].

- Atomic Force Microscopy (AFM): Using the Spheremapper AFM tool to measure equatorial traces on a rotating shell, GA measures (high mode) surface roughness and (low mode) shell distortion to NIC specifications with $1 \mathrm{~nm}$ system noise. Until a few years ago, the Spheremapper sampled only a small fraction of the surface of the capsule, but with recent upgrades, the Spheremapper is now capable of providing complete (19 traces) and accurate measurements of the mid modes.

- Contact Radiography: GA developed a nondestructive technique to precisely profile graded dopants in ICF shells. This quantitative method can detect dopant variation to better than 0.1 atomic \%. Contact radiography also provides accurate dimensional information through the proper corrections of various distortions induced by the imaging lens, the point projection geometry, and x-ray refraction. 


\section{National Ignition Campaign Program Completion Report}

- Scanning Electron Microscopy (SEM): Scanning electron microscopy with energy dispersive xray spectroscopy (EDS) is frequently used for quality assurance and monitoring contamination. GA uses this technique for determining NIC capsule dopant profiles and DU hohlraum microstructure.

- X-ray microscopy: A commercial (Xradia) point projection x-ray microscope is used to measure/characterize the laser drilled fill hole geometry to $\sim 1.5$ micron resolution.

- Energy dispersive x-ray spectrometer (EDS): GA developed a physics-based EDS model and fabricated standards to make it quantitative for low concentration of relatively light elements in a very low-Z matrix to examine NIC capsule contaminants and dopants.

- X-ray Fluorescence (XRF): Commercial XRF systems can only calculate elements atomic percent in flat samples. GA developed a unique XRF program for quantitative XRF computation on spherical samples. This method is accurate to $10 \%$ for high- $Z$ elements and has the trace detection capability at a $1 \mathrm{ppm}$ level for contamination control.

- Precision Radiography: GA designed and constructed a precision radiography system to measure $\mathrm{x}$-ray opacity variation in an ablator capsule to $10^{-4}$ accuracy at 120 -micron spatial resolution. Recent improvement in x-ray tube design enables complete full-surface measurements in one day. This instrument is unique in its ability to see not only the surface perturbations but also the variations caused by non-uniformity of the dopant layers.

- X-ray Edge Absorption Spectroscopy: GA has developed, using the contact radiography setup with an $\mathrm{X}$-ray spectrometer, $\mathrm{x}$-ray absorption spectroscopy of ICF capsules. Measuring the absorption edge can be used to determine the concentration of elements $(Z>17)$ in the presence of other elements, eliminating the "matrix effect" in XRF. It can also be used to determine the thickness of opaque samples, including a 2D map of the thickness variations [16].

- Dual-confocal measurement system: GA has adapted a design from LANL and made it production friendly to provide thickness measurements over a sample area for opaque samples to complement its x-ray edge absorption spectroscopy unit. 3D mapping of ripples and steps in target components to $\sim 1$-micron accuracy has been achieved.

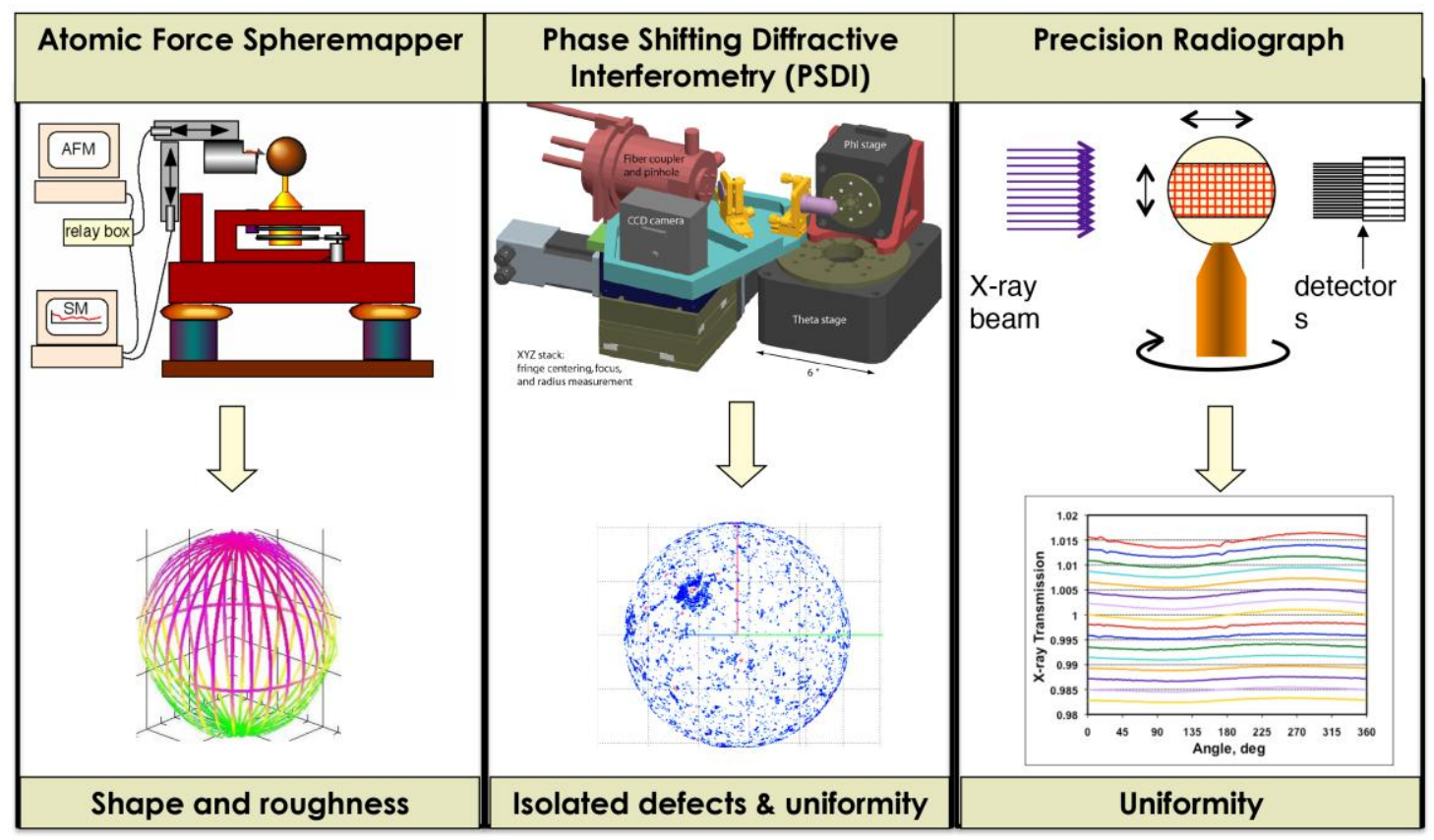

2012-043761s2

Figure 4-10. Various metrology tools are used to determine that capsule specifications are met. From left, Atomic Force Spheremapping facilitates full-surface mapping of capsule shape and roughness, Phase Shifting Diffractive Interferometry is used to look for isolated defects and uniformity, and Precision Radiography is used to confirm capsule uniformity. 


\section{National Ignition Campaign Program Completion Report}

The current target production workforce at GA consists of nearly 90 personnel; of these, approximately 40 are involved in NIC target fabrication. The GA workforce consists of approximately equal numbers of skilled technicians and scientists/engineers. These include precision machinists and engineers involved in development and production of much of the metal components; chemists, chemical engineers, and materials scientists responsible for development of various coatings and processes for capsule fabrication using different ablator materials, including mandrel fabrication; physicists; and optical engineers who ensure development and implementation of proper metrology required for determining the pedigree of the target components. Though most specialize in a single production type, nearly all are cross- trained in several processes. These GA engineers work through various designs with their counterparts at LLNL and then develop and implement the resulting new designs.

\section{Capabilities at LLNL}

In addition to the 3000 square foot Class 100 target assembly area in Building 381, there are two other major LLNL facilities supporting target production, both located in Building 298. These are the Ignition Target Proofing Station (ITPS), used to cryogenically test layering targets prior to sending them to NIF, and the Contaminated Target Repair Facility, used to repair or modify targets which have been tritium contaminated from ITPS or NIF.

LLNL has a suite of materials characterization techniques that have been applied to target metrology and characterization. Characterization techniques/equipment includes:

Atomic Force Microscopy: A molecular imaging atomic force microscope system is used to measure target material surfaces with nanometer spatial and height resolution.

Ion Beam Characterization (Rutherford backscattering, Elastic Recoil Detection Analysis): A $4 \mathrm{MV}$ ion accelerator is used for target research and development, primarily ion implantation doping and ion beam analysis. Ion implantation has been used for doping ICF ablator capsules with ${ }^{124} \mathrm{Xe}$ atoms and potentially other elements of interest to neutron capture experiments. Ion beam analysis experiments use Rutherford backscattering spectrometry and elastic recoil detection analysis for characterization of the elemental composition of many target components, including the ablator, hohlraum, tent, fill tube, and nanoporous foam scaffold. The accelerator has also been used for the development of post-synthesis processing of nanofoams for ICF applications. Less common ion beam analysis experiments have involved nuclear reaction analysis, particle-induced $\mathrm{x}$-ray emission, and ion-induced desorption spectrometry.

Confocal Microscope $4 \pi$ Capsule Inspection System: The cleanliness requirement has led to the development of other special fixtures and microscopy tools, such as confocal microscopy for $4 \pi$ inspection of capsules for particles added during assembly. This system allows particles as small as a few microns to be identified and their location translated to NIF target chamber coordinates for that specific target (see Figure 4-11).

Focused Ion Beam with Scanning Electron Microscopy (FIB SEM): The focused ion beam characterization allows for site-specific analysis of various capsules by conveniently cutting open the thick coating layers and revealing the internal microstructures, defects (if any exist), and composition details. The FIB measurements offer critical feedback for controlling capsule deposition parameters, and help to fabricate compositionally and microstructurally uniform capsules. FIB techniques are ideal for micrometer-scale area analysis and offers good sampling for millimeter-sized capsules.

Transmission electron microscopy with electron energy loss: Transmission electron microscope (TEM) with electron energy loss spectroscopy offers atomic structure level analysis and extremely highenergy resolution for composition analysis of capsule materials. The information offered by TEM allows for measuring the capsule microstructure down to nanometer spatial resolution and is also critical to controlling the doping atom locations within the capsules. The technique further provides scientific insights for improving capsule fabrication. 


\section{National Ignition Campaign Program Completion Report}

Micro x-ray computed tomography: Peering inside optically opaque materials requires penetrating xrays, acoustic waves, or particles. LLNL's x-ray computed tomography system images materials with a resolution of less than 1 micrometer over a 1-millimeter field of view. This technique provides spatiallyresolved opacity that can be translated to density in known compositions and thicknesses.

Double-sided white light interferometer: LLNL developed a double-sided, white light interferometer to scan both sides of a sample simultaneously to provide thickness measurements over the sample area. Combining a "dual roof" mirror and a right angle mirror and a long working distance interferometer objective lens affords equal path length to the front or rear surface of the sample. 3D mapping of ripple and steps in target components to $\sim 1$ micron accuracy has been achieved.

At LLNL, the target group has access to beamlines at synchrotron facilities such as the Advanced Light Source at Lawrence Berkeley National Laboratory, Stanford Synchrotron Radiation Lightsource at SLAC National Accelerator Laboratory, and the Advanced Photon Source at Argonne National Laboratory. These tunable, intense x-ray sources are used to perform scattering, diffraction, absorption (near edge and fine structure), and tomography measurements of target materials and capsules.

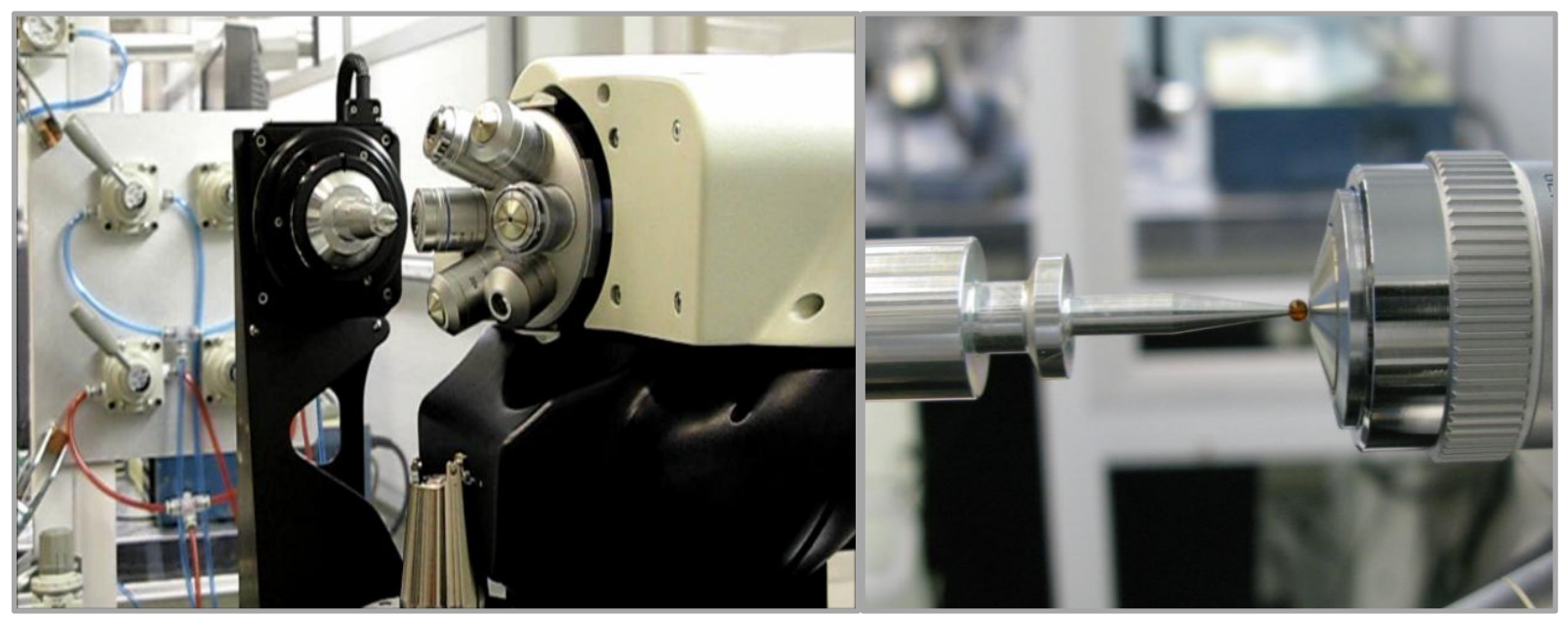

Figure 4-11. Close-up view of a capsule undergoing a near- $4 \pi$ inspection using a confocal microscope.

The current target production workforce at LLNL consists of 21 technicians and 5 production engineers. Technicians are trained for a primary assembly role and may be cross-trained in up to five additional assembly tasks. There are a minimum of two fully qualified technicians for each assembly task and at least one qualified mentor. The work shift is currently eight hours per day, five days per week. The engineering staff ( $\sim 8$ engineers and four technicians) supports target production by providing design, component, tooling, and assembly expertise to ensure an agile production capability that can respond to changing requirements that evolve from the experimental ignition program. In addition, there are approximately 15 material scientists, chemical engineers, and synthetic chemists plus a contingent of postdoctoral students working in more fundamental areas related to target production, such as material development for foams (nanoporous materials) of varying and graded density, metallic nanoporous foams, ablator materials (such as high density carbon (HDC) or boron carbide $\left(\mathrm{B}_{4} \mathrm{C}\right)$ ), doping of fully dense ablators, and doping of nanoporous materials via atomic layer deposition. 


\section{National Ignition Campaign Program Completion Report}

\section{I.4.2-Cryogenic Target System}

The primary scope of work conducted under the NIC WBS Element I.4.2, Cryogenic Target System, includes the systems and processes necessary to insert and position the target in the target chamber, form and characterize the layer, and maintain the layer quality until the experiment is initiated. The main components of the system are the ignition target insertion device, target positioner, hydrogen fill system, layering capability, layer characterization diagnostics, and removable shroud.

\section{A. Cryogenic Target Positioning System}

Two cryogenic target positioners have been fielded in the NIF target chamber are shown in Figure 4-12. They have similar positioning, target gas fill, and cryogenic capabilities. However, one of the positioners is configured for rapid-turnaround of warm and cryogenic shots (TARPOS 90-239, often referred to as TARPOS) and the second for layering (TARPOS 90-015, often referred to as CryoTARPOS).

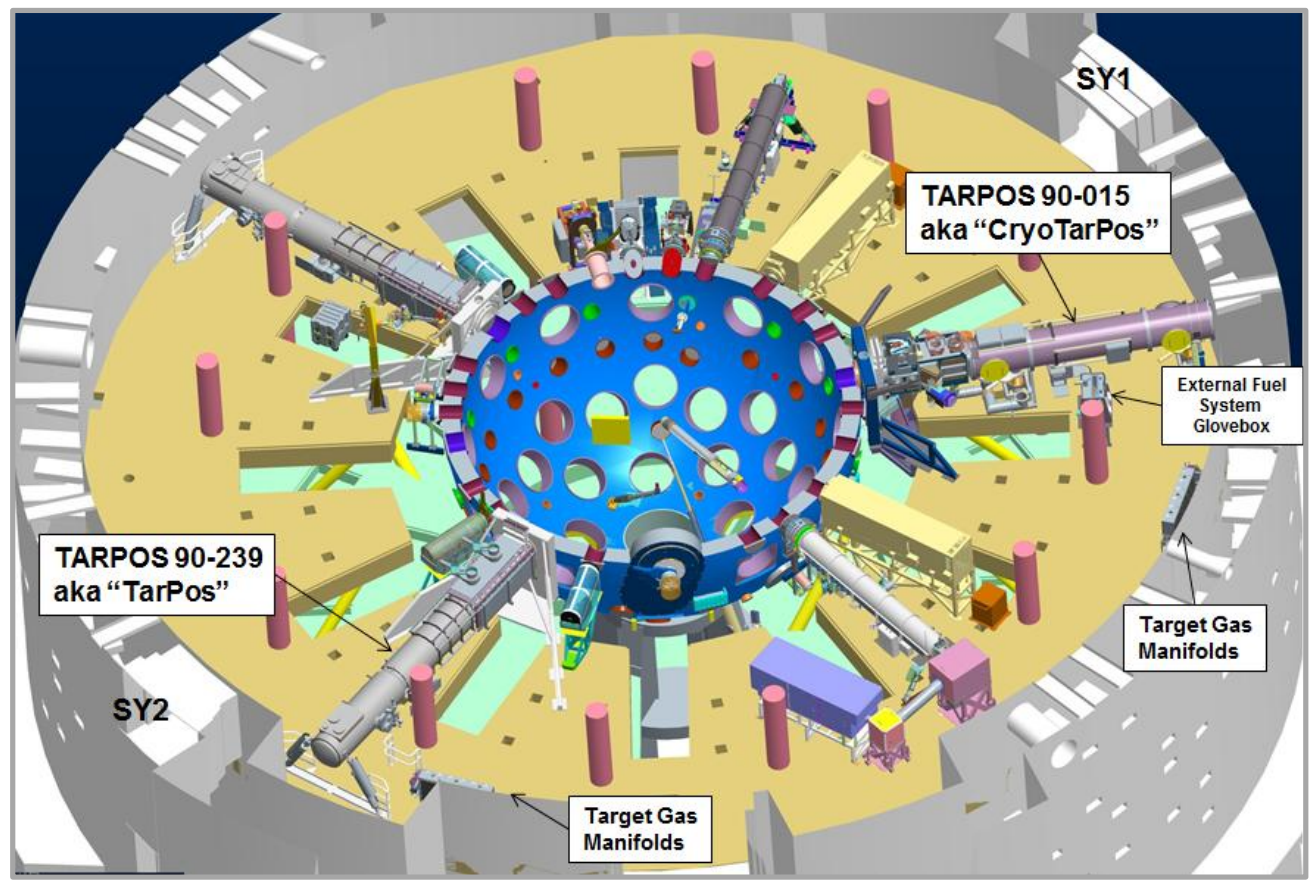

Figure 4-12. TARPOS 90-239 is capable of cryogenic and warm shots. TARPOS 90-015 has additional capabilities for layering and gas fills of $\mathbf{T}_{2}, \mathrm{DT}$, and THD.

The Cryogenic Target Positioning System (CTS), also called CryoTARPOS, consists of four primary components: 1) the Target Positioner; 2) the Ignition Target Inserter Cryostat (ITIC); 3) the Load, Layering, and Characterization System (LLCS); and 4) the gas fill system. Together, these elements enable the placement and maintenance of a well-characterized, cryogenically layered fuel capsule at the TCC [17]. The three integrated components of the CTS (positioner, ITIC, and LLCS) are shown schematically in Figure 4-13. TARPOS (TARPOS 90-239) will not be discussed here in detail. TARPOS was originally fielded as a room-temperature target positioner as part of the NIF Project but has been retrofitted to be functionally similar to CryoTARPOS except that TARPOS:

- Does not include the LLCS or a dedicated high vacuum system, so it cannot be used for layering.

- Uses a Non-ignition Target Insertion Cryostat (NTIC) instead of an ITIC to reduce cooling time by four hours to expedite shot turnaround. 


\section{National Ignition Campaign Program Completion Report}

- Uses gas delivery, ventilation and alarm systems that are limited to a relatively low inventory of tritium (appropriate for "pusher" targets-100 $\mathrm{mCi}^{\mathrm{t}}$ ) rather than the level of several curies required for layering targets.

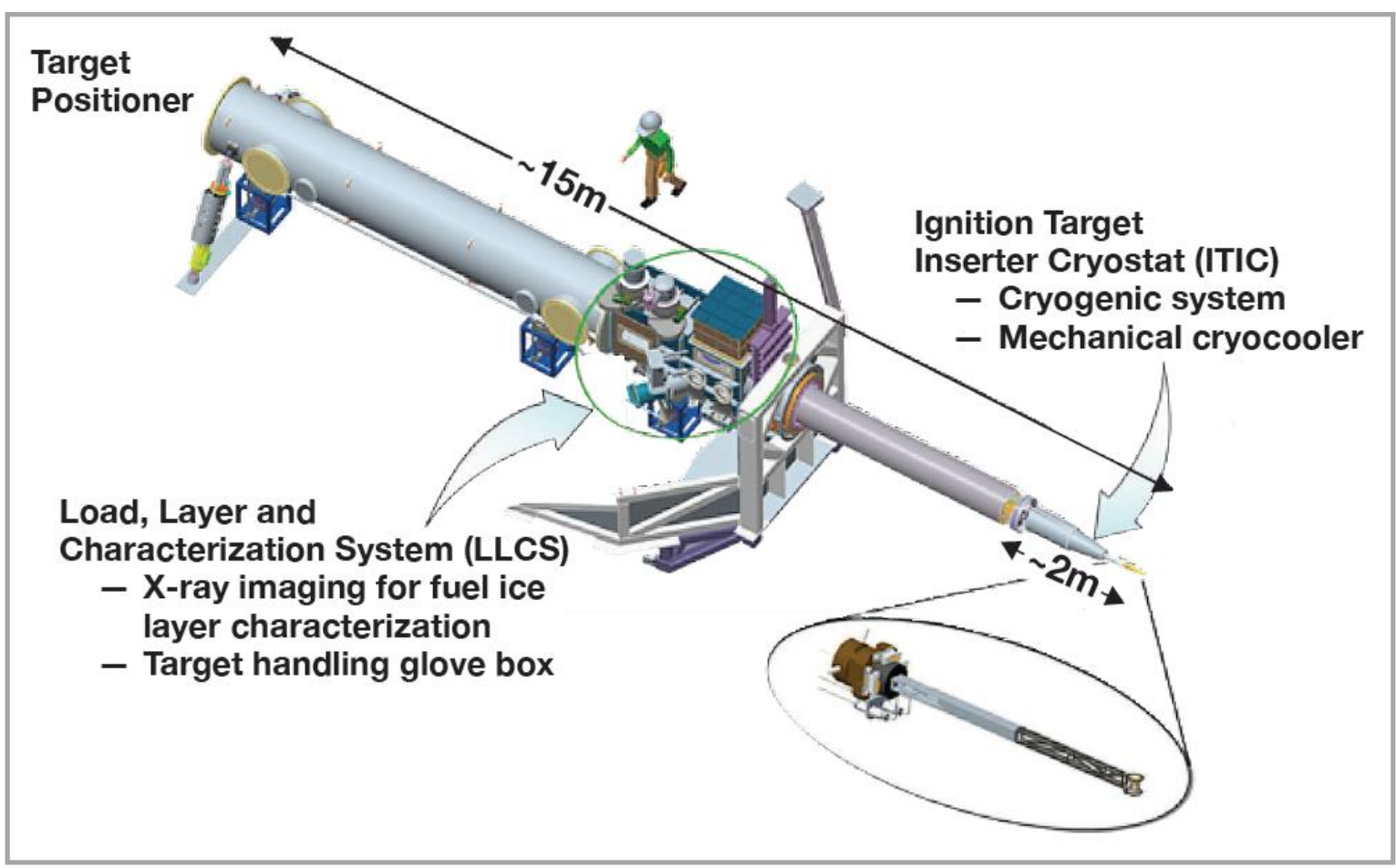

Figure 4-13. The cryogenic target positioning system performs the complex tasks of refrigeration, hydrogen layer growth and characterization, and target positioning.

\section{Cryogenic Target Positioner}

The target positioner element of CryoTARPOS is used to place the target at the center of the spherical, 10-meter diameter NIF target chamber. Its design was based on a room-temperature version of TARPOS fielded for NIF Early Light ${ }^{\mathrm{u}}$ shots in 2003. The body of the positioner consists of a stainless steel cylindrical vacuum vessel. Inside the vacuum vessel is a 0.5 -meter diameter, 7-meter long boom constructed of carbon composite, a material with a low thermal expansion coefficient. The boom rides on linear rails, and the positioner incorporates five degrees of freedom to adjust the position of the target. Adjusting the stroke of the boom along the rails controls the target's position along its axis. Lengthening or shortening the struts that support the rear of the vacuum chamber adjusts the two transverse directions. Two rotations of the nose cone, roll and nod, are adjusted by a geared mechanism at the end of the boom (see Figure 4-14). Incremental adjustments and positional feedback enable the precise positioning of the target.

$\mathrm{t}$ The curie (symbol Ci) is a non-SI unit of radioactivity and is roughly the activity of 1 gram of radium-226. Curies are often used to express a quantity of radioactive material rather than a decay rate such as $1 \mathrm{Ci}$ of tritium.

u In 2003, the NIF Early Light beampath was completed and commissioning teams achieved a major objective of propagating one quad of NIF beams "first light" to target chamber center. At that time, target experiments were conducted to exercise the system and to apply value engineering principles to complete the design and construction of the remaining 188 beams. 


\section{National Ignition Campaign Program Completion Report}

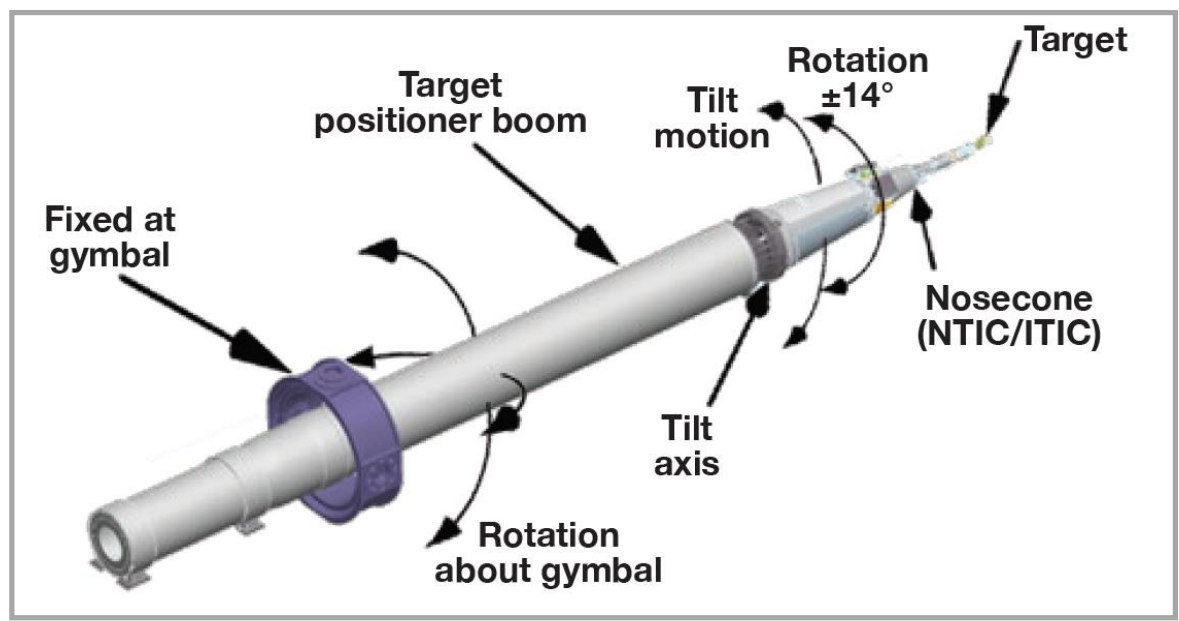

Figure 4-14. Diagram of the target positioner showing the degrees of freedom for positioning a target.

The error budget for laser-on-target positioning has allocated $7 \mu \mathrm{m} \mathrm{RMS}^{\mathrm{v}}$ to positioning of the target. A potential source of positioning error is vibration, which has been measured on the room temperature TARPOS to be approximately $1.5 \mu \mathrm{m}$ RMS. Target positioning with the CryoTARPOS (see Figure 4-15) is much more complex due to the systems required to cool the target and to expose the target to the laser a few seconds before the shot. The cryogenic system is designed to eliminate cryocooler-induced vibration by temporarily shutting down the cryocooler while maintaining target temperature. During critical steps in the target fielding process, the CryoTARPOS vibration can be made nearly identical to that of the room temperature TARPOS. The remainder of the target positioning error budget is allocated to longer-term positional drift of the target while at the center of the target chamber.

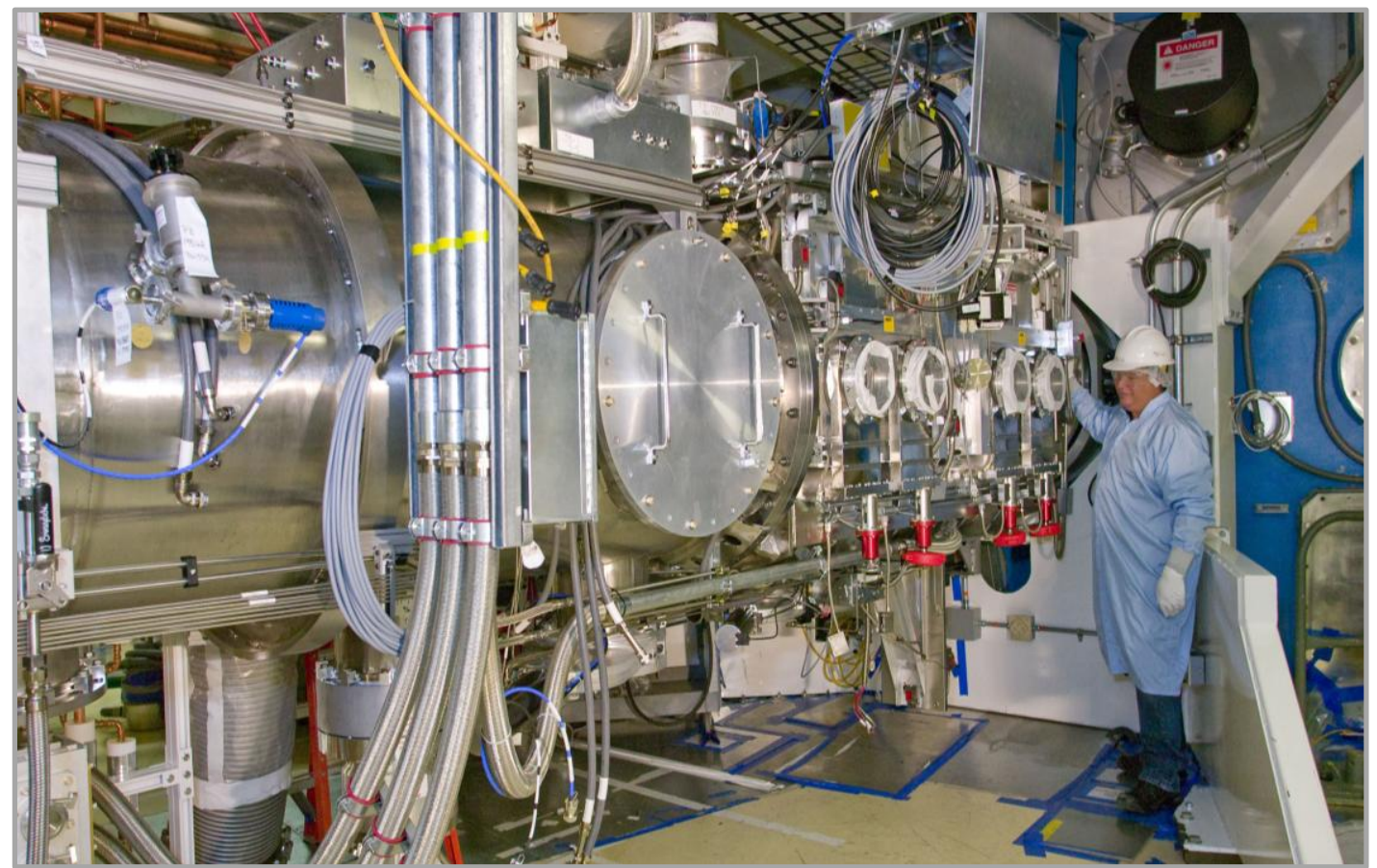

Figure 4-15. Picture of the CryoTARPOS located on NIF port $(90,15)$ during commissioning in 2009.

\footnotetext{
${ }^{\mathrm{v}} \mathrm{RMS}=$ root-mean-square
} 


\section{National Ignition Campaign Program Completion Report}

The positioner also holds the target during DT fuel ice layer formation (the process known simply as layering) [18]. The stability requirement, dictated by ice layer characterization requirements and the x-ray imaging system, is $\pm 1 \mu \mathrm{m}$ during the 6 second $\mathrm{x}$-ray image frame integration time.

\section{Ignition Target Inserter Cryostat}

Mounted at the end of the boom, the ITIC (shown in Figure 4-16) is used to cool the target from room temperature $(\sim 293 \mathrm{~K})$ to temperatures just below the DT triple point ${ }^{\mathrm{w}}(18 \mathrm{~K})$. Once cooled, multiple control points, consisting of pairs of temperature sensors and heaters, are used to maintain the target hohlraum temperature with a precision of $\pm 0.001 \mathrm{~K}$. The ITIC also ensures that all structures influencing target position are maintained at room temperature or are controlled at a stable intermediate temperature.

The ITIC cryogenic system consists of the cryocooler connected to the cryogenic thermal capacitance device, followed by a coaxial structure reaching to the target base. The innermost conductive path, called the cold rod, is connected to the second stage of the cryocooler and operates near $4 \mathrm{~K}$. Surrounding the cold rod is a $60 \mathrm{~K}$ thermal shield that limits heat transfer to the cold rod and is connected to the first stage of the cryocooler. Surrounding the $60 \mathrm{~K}$ cold shield is a warm shield that is controlled to ambient temperature using heaters. The cold rod and cold shield are fabricated using oxygen-free, highconductivity copper and are gold plated to reduce radiation heat transfer.

The ITIC cryogenic system is packaged into the ITIC structure. Ignition targets are installed on the ITIC gripper at room temperature by operators working in the LLCS vessel, shown in Figure 4-17. A two-layer thermal shroud surrounds the target, as shown in Figure 4-16. The inner layer of the shroud is thermally connected to the cold shield and operates at about $100 \mathrm{~K}$; the inner shroud minimizes both thermal radiation and condensation on the target. To minimize cooling of nearby equipment, the outer layer is controlled to $293 \mathrm{~K}$. The shroud opens in a clamshell manner a few seconds before the shot to expose the target to the laser. Structural analysis and laboratory tests have been performed to ensure that the vibration imparted by the opening of the shroud reaches acceptable levels before the shot.

To minimize vibration disturbance, the NIF CryoTARPOS uses a thermal capacitance device that permits the cryocooler to be turned off during periods requiring positioning stability. X-ray imaging of the ice presents the most difficult challenge. The cryogenic system must maintain target temperature within \pm 1 $\mathrm{mK}$ with the cryocooler off for the duration of the x-ray imaging time, despite the fact that most engineering materials lose almost all of their heat capacity at temperatures below $100 \mathrm{~K}$. The ITIC cryogenic system uses high-pressure helium confined in a copper structure that minimizes the heat transfer time constant by ensuring that all helium is within a few millimeters of a copper conduction path. The temperature stability was demonstrated over a 48-hour period with a 4 minute off and 6 minute on duty cycle during commissioning.

\footnotetext{
${ }^{\mathrm{w}}$ The temperature and pressure at which the three phases (gas, liquid, and solid) of that substance coexist in thermodynamic equilibrium.
} 


\section{National Ignition Campaign Program Completion Report}

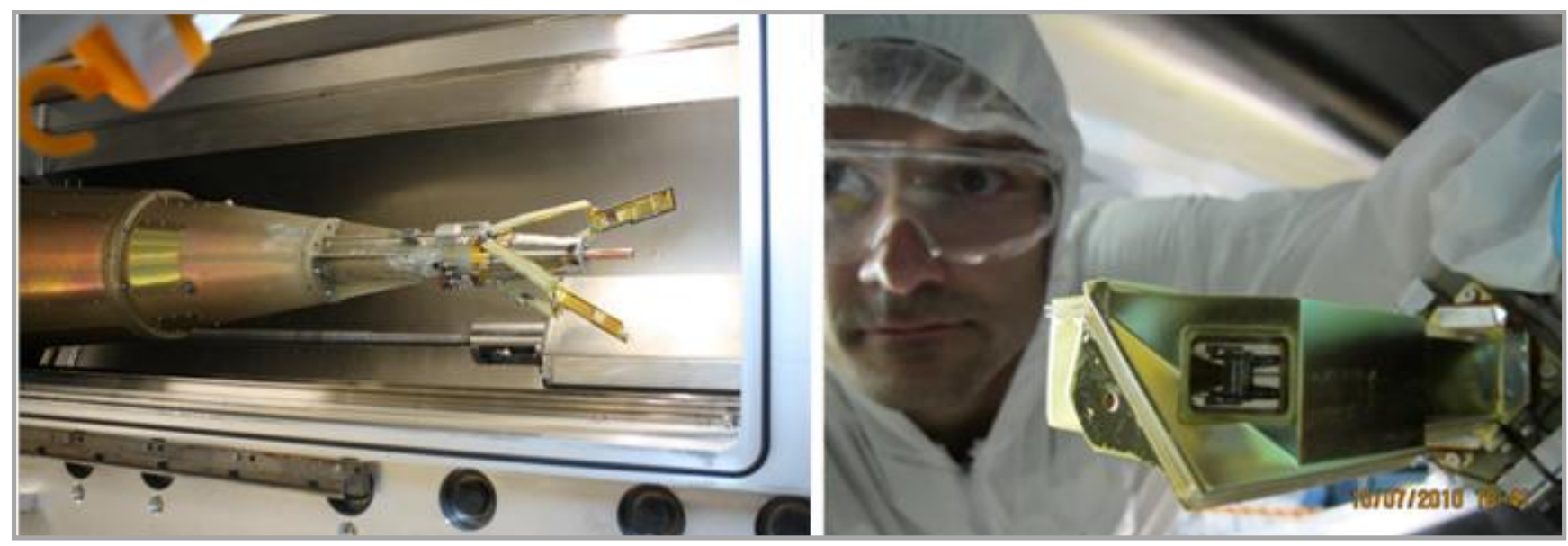

Figure 4-16. (Left) Target loaded on the ITIC, located at the end of the target positioner. The heat shrouds surrounding the target are shown partially retracted. (Right) View of a target through the alignment windows of the shrouds that protect the target from its thermal environment.

\section{Load, Layering, and Characterization System}

Loading the DT fuel into the target and cooling, forming, and characterizing the layer prior to TCC positioning is performed using the LLCS and the gas fill system. During installation or removal of a target, the vessel serves as a ventilated enclosure. In this mode, the vessel is connected to the NIF Diagnostic and Positioner Ventilation system and the operators work through the side door as seen in Figure 4-16. When high-Ci reservoir filling is required, the LLCS can perform as a glove box as shown in Figure 4-17.

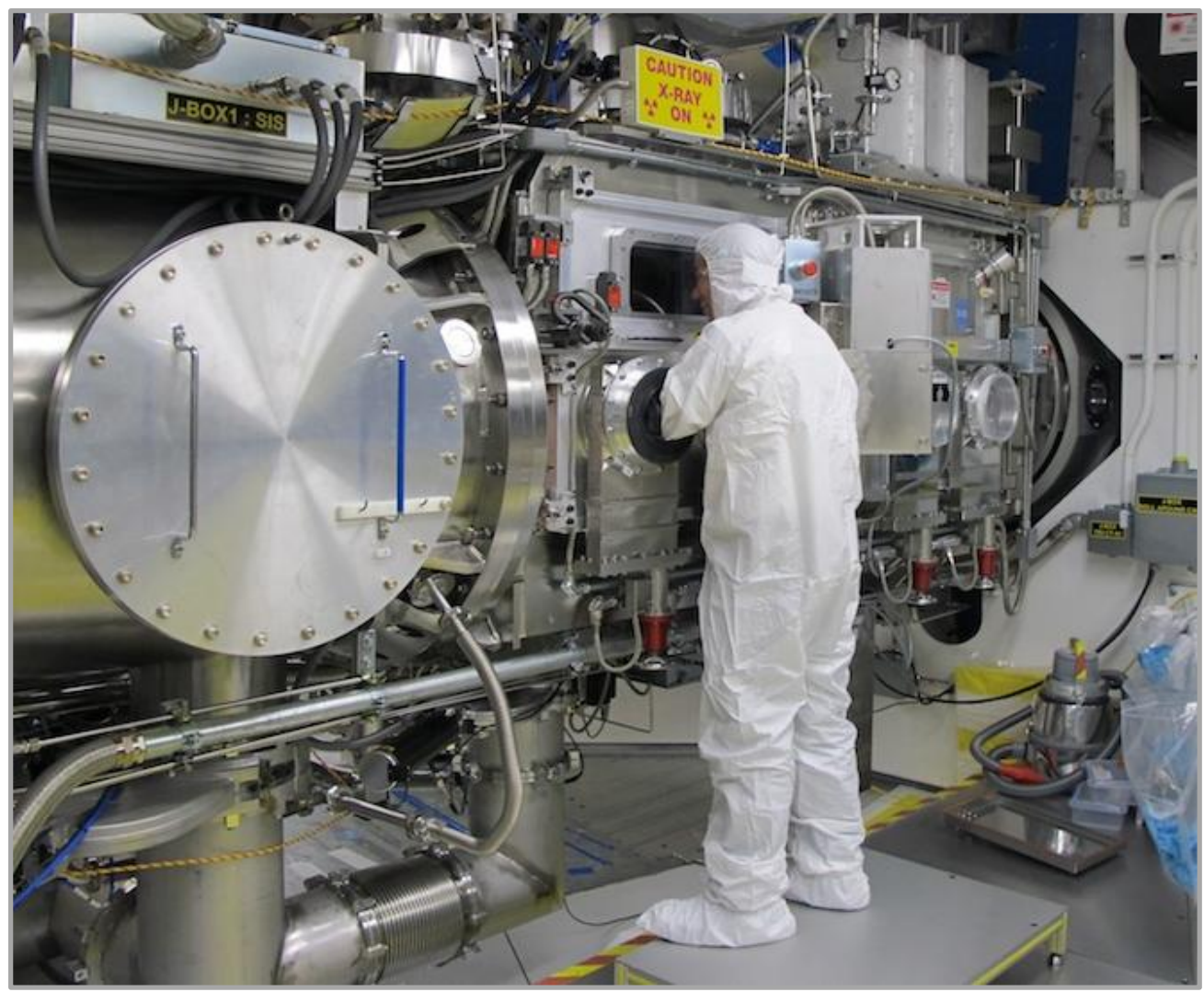

Figure 4-17. LLCS with large door closed so that a high-Ci reservoir can be installed directly onto the ITIC. 


\section{National Ignition Campaign Program Completion Report}

LLCS x-ray characterization equipment measures the smoothness (surface roughness) and uniformity (thickness, roundness) of the DT ice layer within the spherical capsule. Solid hydrogen is very transparent to $\mathrm{x}$-rays at the $\mathrm{x}$-ray wavelengths needed to penetrate the plastic shell. Therefore, traditional $\mathrm{x}$-ray imaging cannot be employed to image the DT fuel layer. The very small deflection of the x-rays at the hydrogen surface, however, can be used to characterize the fuel layer surface. This is known as phasecontrast enhanced $\mathrm{x}$-ray imaging and requires an $\mathrm{x}$-ray source with a point-like emission and placing the detector at a sufficient distance to allow the small angular deflection of the x-rays to be observed [19]. Three such x-ray imaging systems are employed in the LLCS, one imaging through the laser entrance hole of the hohlraum, and two through the sides of the hohlraum (Figure 4-18). The three views are necessary to determine the out-of-roundness of the fuel layer (low-mode errors). With this information, and precise control of the hohlraum temperature profile, both the layer offset from the center of the ablator shell and the layers ellipticity can be minimized in the LLCS. The three x-ray views allow characterization of the quality of the ice layer relative to other ignition requirements, including size and number of grain boundaries, and discrete defects in the ice layer.
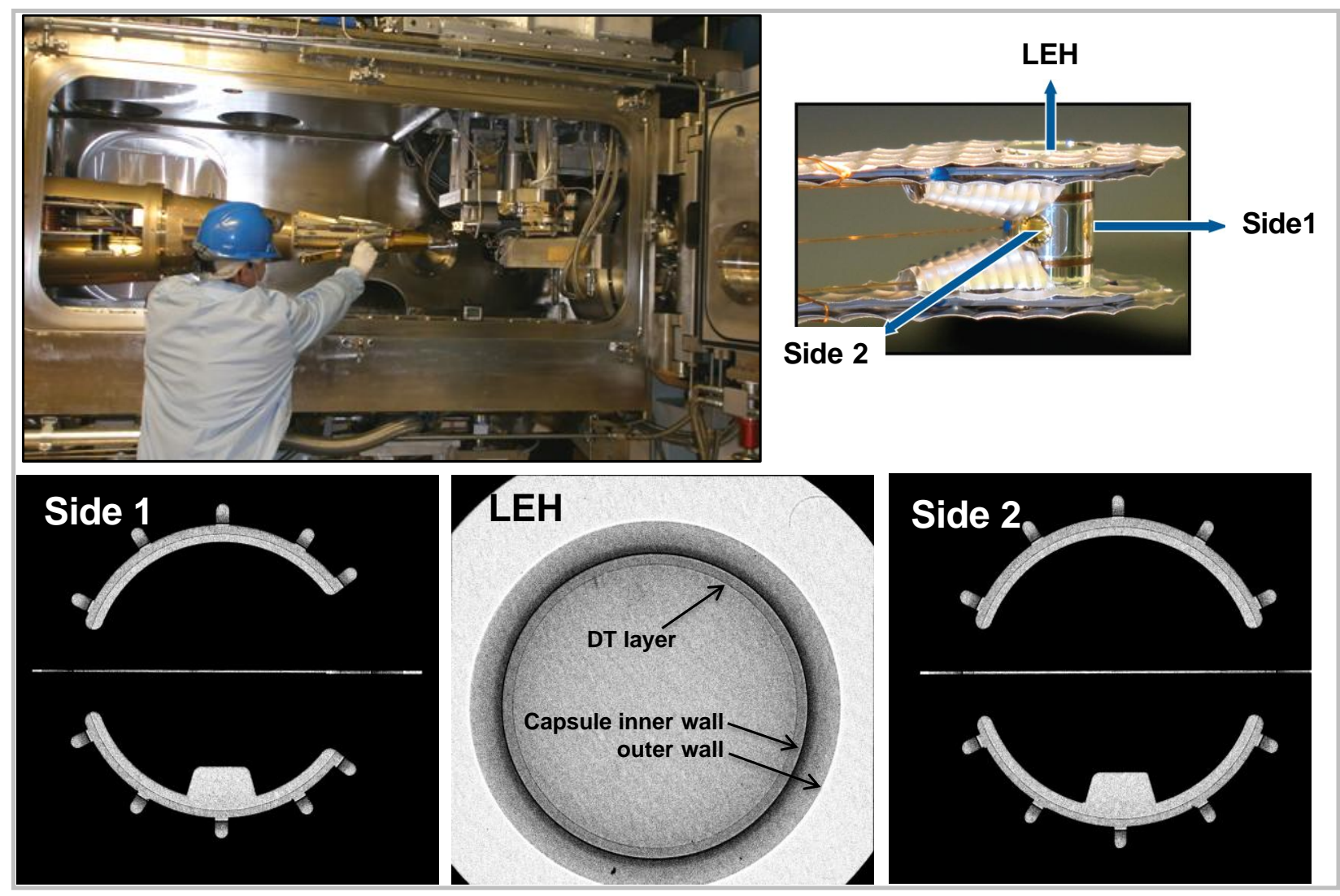

Figure 4-18. (Upper left) A technician finishes the insertion of a target into the LLCS prior to evacuation, cooldown, layer formation, and characterization. (Upper right and lower) X-ray images taken from three directions are used to view and evaluate ice layer shape, smoothness, and grooves.

\section{B. Gas Fill System}

The gas fill system has three components: the Target Gas Manifolds (TGMs) mounted on the wall of the Target Bay, an external glove box mounted near the CryoTARPOS, and a valve manifold mounted directly on the nose of the ITIC in a cluster of three small air boxes. These are shown in Figure 4-19. The TGM, identical for both TARPOS and CryoTARPOS, is used to pump and purge air from the target and perform portions of the target filling. The TGM removes air before the target reaches a temperature where air would freeze (between 32 to $60 \mathrm{~K}$, depending upon fill pressures) and fills the hohlraum with the 


\section{National Ignition Campaign Program Completion Report}

desired fill gas—usually ${ }^{4} \mathrm{He}$. The TGM accomplishes these tasks using long, flexible tubing — nearly 30 $\mathrm{m}$ from the Target Bay wall through the positioner.

For Symcap or other low-Ci shots, the TGM also provides the fill gas for the capsule (D- ${ }^{3} \mathrm{He}, \mathrm{D}_{2}$, etc.). A series of pump/purge cycles is used to reduce air and other condensable gases to parts-per-million levels to avoid freezing in the capsule fill tube or onto the capsule and to avoid interfering with layering.

Layering requires high-Ci gas fills, generally 50:50 DT or variations of THD. These fills are accomplished using small reservoirs (typically $1.7 \mathrm{cc}$ ) that are pre-filled at the LLNL Tritium Facility and are installed either in the external glove box or directly onto the ITIC gas manifold (using the glove box mode of the LLCS), depending on specific needs of the experiment. These two reservoir systems are also used for high-Ci gas fills for non-layered targets that exceed the tritium inventory limits of TARPOS (examples include DT and $\mathrm{T}_{2}$ gas-filled Symcap targets).

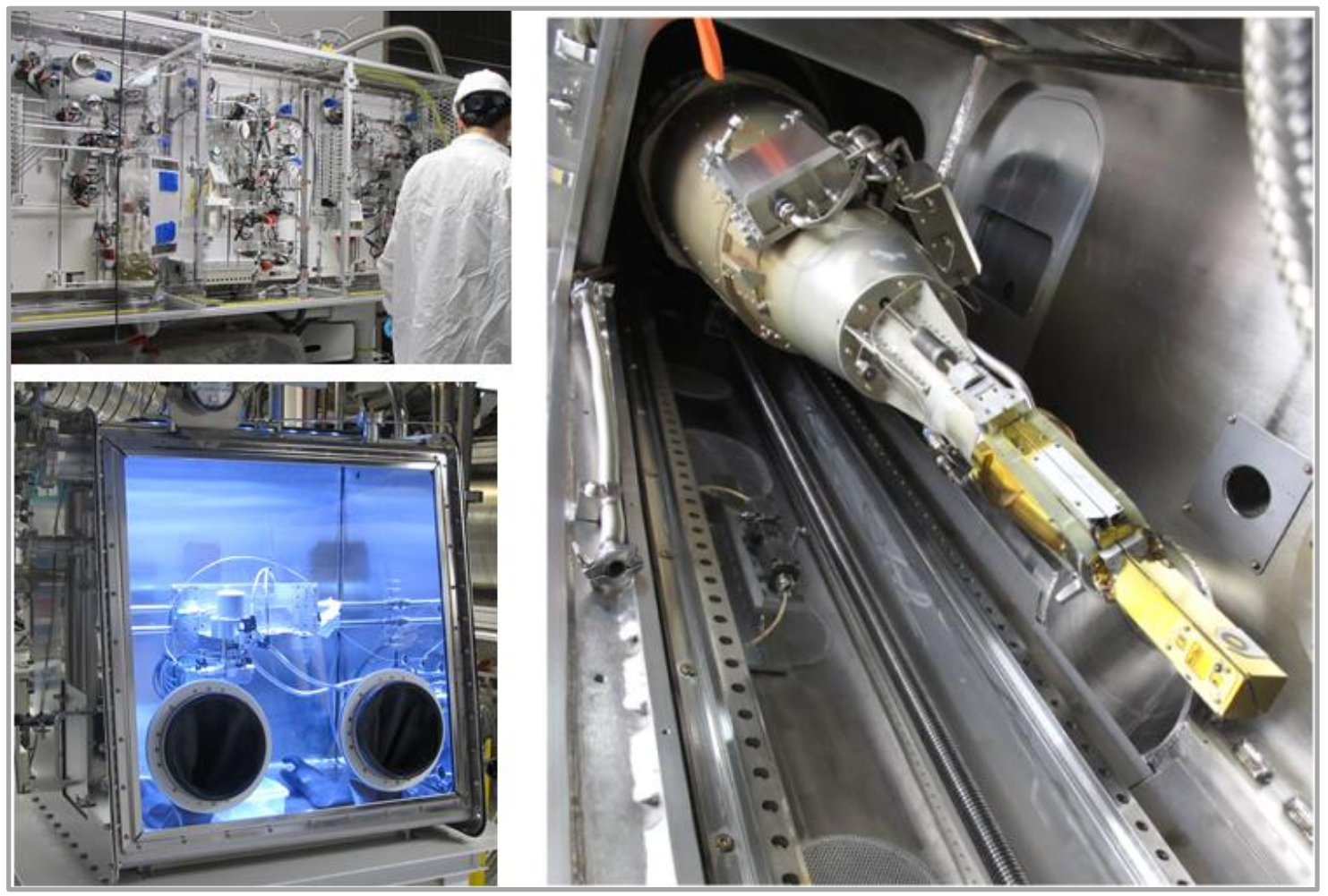

Figure 4-19. (Upper left) Target Gas Manifold on the wall of the Target Bay used to remove air from targets and DT reservoir connections and to fill the hohlraum with ${ }^{4} \mathrm{He}$. (Lower left) External glove box used to fill the capsule with high-Ci fills such as DT, THD, or $T_{2}$ (Right) View of the ITIC showing the ITIC gas manifold-three small air boxes that enclose valving and a pressure gauge for managing the layering gas fill after the target reaches cryogenic temperature of $\sim 18 \mathrm{~K}$. In this photo, a target is installed but not visible inside the closed shrouds.

\section{Cryogenic Layering}

Capsule layering, a major technical and scientific challenge, was successfully addressed during the NIC. The process for growing spherical, ultra-smooth DT and THD layers free of even very small isolated defects has been refined over many years [20]. The low-mode sphericity requirement has been achieved by using heaters to produce a symmetric thermal environment within the capsule during fuel (DT) crystallization. The roughness and isolated defect specifications are met by growing the crystal layer with only small low-angle grain boundaries.

Over the past few years, scientists at LLNL and LLE have achieved high-quality layers in approximately one out of every three crystal growth attempts. Roughly a third of the layers that do not meet specification meet the roughness requirement but have isolated defects that exceed the NIC specification by up to three 


\section{National Ignition Campaign Program Completion Report}

times. The remaining layers not meeting specification have much larger isolated defects or macroscopic structures. Given the stochastic nature of the crystal seeding process, NIC cryogenic layering success has focused on rapidly characterizing, quantifying, and predicting layer quality. It takes 14-18 hours to grow an acceptable layer, but by catching an unacceptable layer early in the process, the layer can be melted and restarted; this enables up to three growth attempts within a 30 hour time period. The point design calls for $\sim 70$ micron thick condensed layer of a mixture of hydrogen isotopes ${ }^{\mathrm{x}}$ with approximately 1 micron thickness uniformity inside a nominal $2 \mathrm{~mm}$ diameter hollow spherical capsule.

The NIC layering process was developed using specialized layering stations and specialized targets that violate many of the requirements for ignition, but that provide better diagnostic access to the layer. These off-line layering facilities culminated in a NIF-like system that exercised most of the techniques required in NIF including compatibility with NIC ignition targets, use of the NIF three-view X-ray diagnostics, use of the NIF cryogenics system, and NIF integrated controls and software systems. This laboratory layering station, the ITPS, was used for final process and software development prior to moving the NIC layering process into NIF in 2010. The system, shown in Figure 4-20, is in continuous use to proof NIC layering targets prior to use on NIF, for process improvements, and for software automation development.

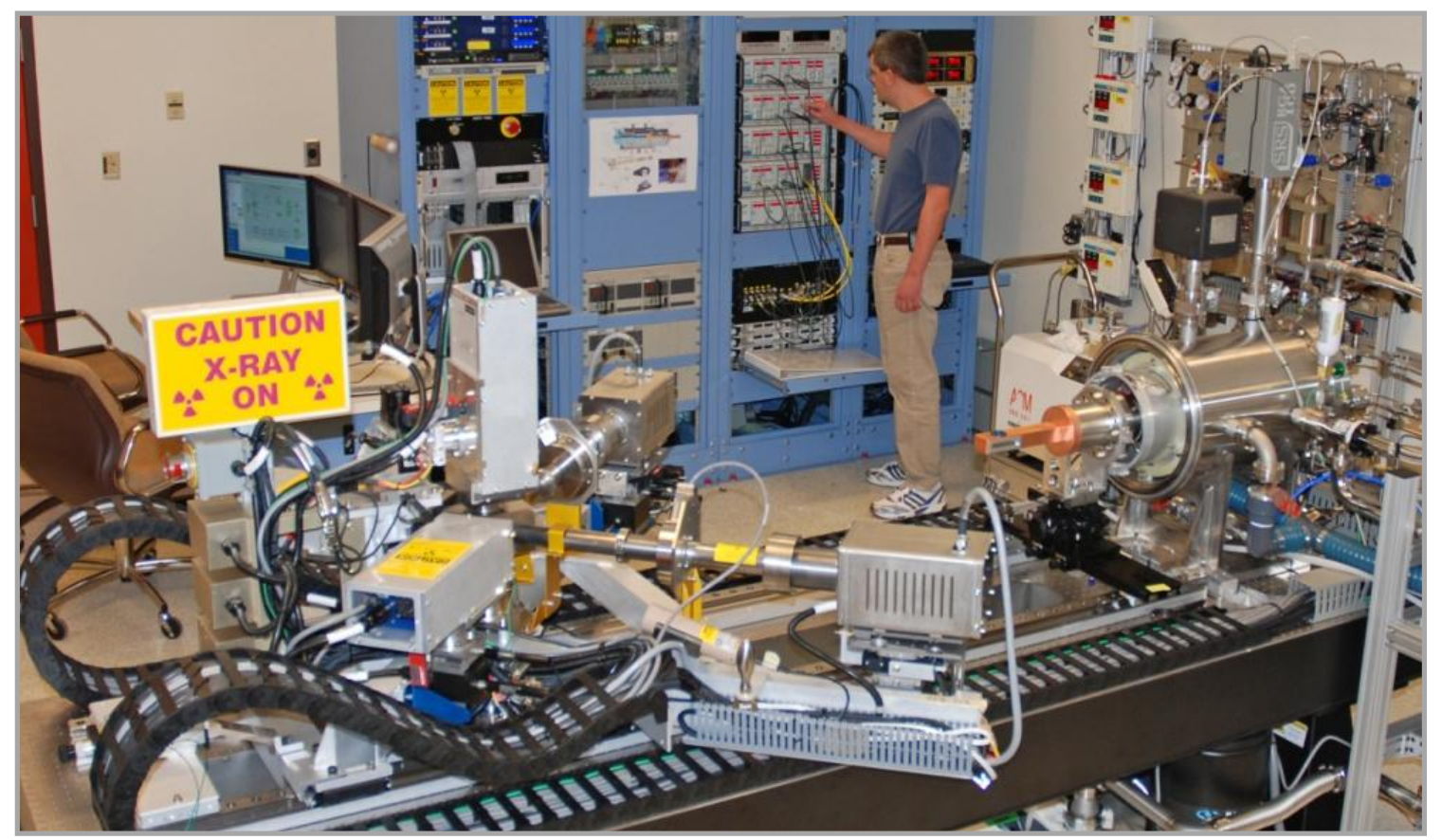

Figure 4-20. The Ignition Target Proofing System is an off-line laboratory facility used for process and software development of layering. Because it uses the NIF cryogenic, $x$-ray, and control systems, techniques developed here are easily translated to NIF.

The layering process is conceptually divided into three major steps:

1. Filling an evacuated capsule with a hydrogenic fuel;

2. Redistributing the condensed fuel uniformly on capsule walls followed by subsequent crystal growth of a frozen fuel layer; and

3. Characterizing the layer uniformity before the implosion.

${ }^{\mathrm{x}}$ The ignition point design capsule uses an equimolar (50:50) DT mixture; however, some NIC experiments use fills consisting of other isotopic mixtures where normal hydrogen $(\mathrm{H})$ replaces some amount of the deuterium (D) in the fuel. The fuel for these experiments is referred to as a THD fill. Hydrogen and deuterium are stable isotopes but tritium is radioactive decaying by the emission of a $\beta$-particle. 


\section{National Ignition Campaign Program Completion Report}

\section{Capsule Filling}

A fill tube, shown in Figure 4-21, is used to fill the NIC ignition capsule with fuel. The fill tube material and dimensions are determined based on target design simulations to minimize fill tube effects on the target performance during the implosion. The capsule fill tube is nominally $10 \mu \mathrm{m}$ in diameter, although recent ignition targets have used fill tubes half that size. Prior to the introduction of the fuel, the capsule wall has a few monolayers of residual air molecules (oxygen, nitrogen, etc.) sticking to the inner wall. These atoms, if incorporated in the fuel, act as contaminants and can cause problems in the layering process. This situation is mitigated by purging the target (filling and evacuating repeatedly with deuterium gas) before purified tritium $\operatorname{gas}^{\mathrm{y}}$ is added. A cold trap is incorporated into the base of the target as the final defense to ensure that no condensable contaminants reach the target from the gas handling systems (for layering, this includes any molecule other than isotopes of helium or hydrogen).

The tritium gas is physically located only a few meters from the actual capsule to be filled. However, since the target containing the capsule must eventually be extended into the center of the NIF target chamber (an 8 meter movement), the tritium-containing gas must travel through a long capillary tube in order to reach the capsule. The large inner surface area of the capillary tube, like the inner wall of the capsule, must also be purged to remove potential contaminants (helium, oxygen, nitrogen, etc.). In addition, the capillary tubing must be resistant to the effects of low energy $\beta$-radiation due to tritium decay. The hydrogen pressure is increased to a value needed for filling capsules to the required total number of hydrogen molecules.

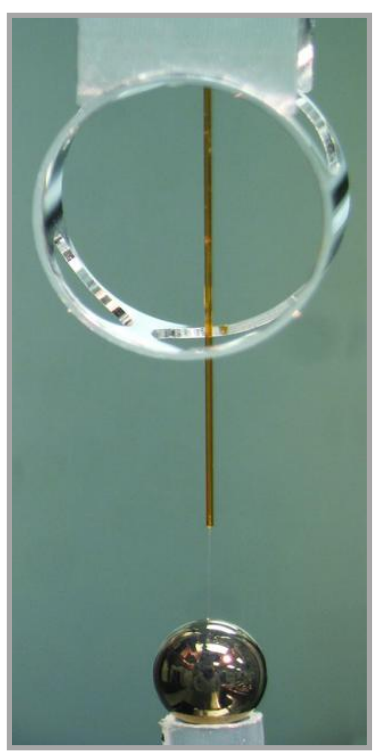

Figure 4-21. A fill tube smaller than a human hair is used to transfer DT fuel to the $2 \mathbf{~ m m}$ diameter target capsule.

In addition to the ablator capsule, critical components include miniature temperature sensors that record continuously relevant parameters. The liquid hydrogen capsule fueling is monitored by $\mathrm{x}$-ray imaging and is terminated by rapidly cooling the fill tube, resulting in the formation of a solid hydrogen plug inside it. Such a solid plug effectively isolates the fuel delivered to the capsule from that left in the reservoir connected to the capsule via the fill tube. A challenge of fill tube filling is accurately controlling the total amount of hydrogen in the capsule, which determines the thickness of the resultant condensed layer. A second challenge is achieving the desired molecular composition of the fuel. This is accomplished by carefully mixing pure isotopes and verifying the resulting composition with mass spectroscopy in the LLNL Tritium Facility (B331) and by measuring the melting point of the mix inside the capsule each time a layer is grown.

\section{Fuel Layering}

Cryogenic layering often refers only to the process of redistribution of condensed hydrogen fuel on the inner wall of the capsule. After filling, liquid hydrogen perfectly wets the capsule wall and, under gravity, forms a puddle at the capsule bottom. When cooled below the triple point, the liquid puddle converts to a solid ice layer. Well-known crystal growth principles and techniques are applied for nucleation and subsequent growth of hydrogen crystals. In addition to miniature temperature sensors, miniature heaters are also incorporated near the cryogenic capsule, creating a spherically symmetric thermal environment.

${ }^{\mathrm{y}}$ Because tritium is radioactive, it decays to helium-3 by emission of a $\beta$-particle with a half-life of 12.3 years. Helium-3 is a gas that accumulates at a rate of about 150 part per million (ppm) per day and can also act as a contaminant causing problems during layering. Therefore, tritium is purified (the helium-3 is removed by gas purging) before the NIC capsule is filled. 


\section{National Ignition Campaign Program Completion Report}

Hydrogen redistributes uniformly across the capsule wall via evaporation of warmer thicker regions and condensation onto colder thinner regions.

The hydrogen (DT) ice layer solidifies in a crystalline lattice. By definition, a crystal is a uniform arrangement of atoms, with every atom equally spaced from its neighbor. A perfect single crystal of any material is difficult to form, and hydrogen is no exception. The roughness of a typical solid DT layer is the result of complex crystallization and polygonization processes and is caused by grain boundaries. (A grain boundary occurs at the intersection of two distinct and separate crystals.) Faults in the crystal structure that form during growth of the crystal will propagate with the growth and result in slight misorientation of the crystal grains. A groove in the crystal surface forms when the grain boundary reaches the solid surface. It is the random nature of the crystal growth seeding process and subsequent formation of grain boundaries that potentially causes an individual layering step to exceed the required smoothness specifications. Layers with the smoothest surfaces result from the growth of an initial single seed crystal. The key to the success of the NIC cryolayering effort is to first attempt to form a layer from a single crystal, then slowly solidify the layer to minimize the number of defects that occur during formation of the layer. Next, the layer is monitored during growth to assess the size, number, and location of the grain boundary grooves (during crystallization). If the layer quality becomes unacceptable, growth is halted, and begun again within the time period set by the tritium decay rate (avoiding the accumulation of helium in the capsule, the decay product of tritium).

\section{Fuel Layer Imaging and Characterization System}

Diagnosis and verification that the cryogenic layer meets specifications is nearly as challenging as growing the layer itself. Major challenges include:

1. Operating within a cryogenic environment where small amounts of heat from the diagnostics can disrupt the layering process;

2. Characterizing a low atomic number $(\mathrm{Z}=1)$ cryogenic fuel layer that is surrounded by a denser plastic ablator shell; and

3. Diagnosing a capsule located inside a cylindrical, high $\mathrm{Z}$ hohlraum made of gold ( $\mathrm{Au})$ or depleted uranium (DU).

The layering process used in NIF was developed using customized layering targets and laboratory systems that employ multiple diagnostics to characterize the ice layer uniformity - optical microscopy, $\mathrm{x}$ ray radiography, and optical interferometry (Figure 4-22). All three methods were applied to characterization of fuel layers. Optical microscopy has proven to be a powerful technique for monitoring the evolution of the layering process such as seed nucleation and propagation, the transport of liquid hydrogen due to the formation of new solid/liquid interfaces during crystallization, and the formation and evolution of grain boundaries. Optical interferometry was used to study the morphology of the hydrogen solid-vapor interface. Qualitative analysis of the layer quality was performed by analyzing onedimensional traces of the hydrogen ice layer thickness and describing the average layer thickness and concentricity. 


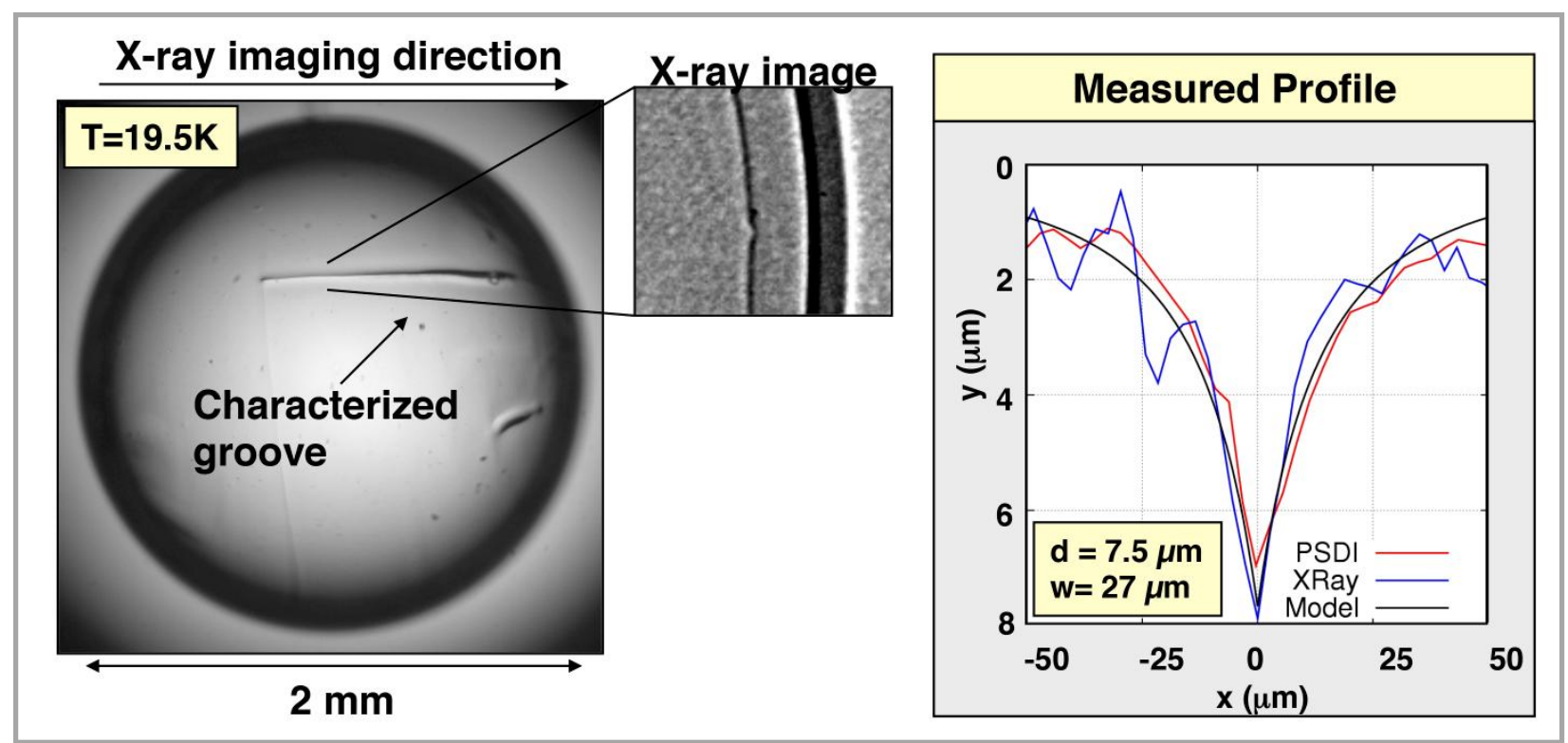

Figure 4-22. Example of characterization information obtained using a combination of optical and x-ray techniques on the DT layer in the offline laboratories using customized non-NIF targets.

The NIC ignition target and the NIF Target Bay environment make layering characterization more challenging than in the off-line laboratories. In the LLCS, only the three-view phase-contrast X-ray imaging technique is available. Knowledge from the diagnostically richer laboratory experiments enabled creation of the NIF layering process using a more limited set of layering diagnostics - the three x-ray views shown in Figure 4-23. Two views (Side 1 and Side 2) of the target can be obtained using slits in the equatorial plane of the hohlraum wall. A third view is possible vertically through the circular LEHs of the hohlraum. Each imaging axis incorporates a micro-focus x-ray source enclosed in an air box in close proximity to the target. All three air boxes are arranged on a single strongback that can be raised up to allow the boom to stroke into the NIF target chamber. X-ray CCD cameras are used, and these CCD cameras are located outside the LLCS vacuum vessel. Beryllium windows enable the X-rays to pass through the vacuum vessel wall.

Current specifications for the layer quality of the NIC hot spot ignition target focus on layer roughness and grain boundaries. The three x-ray views enable near-real-time determination of layer quality with high confidence. A real-time dashboard summarizing layer quality during the growth process is available to operations technicians in the NIF Control Room (Figure 4-23). The ability to make an early decision regarding the layer quality and whether to abort and restart the ice layering process increases the shot rate for layered implosions. 


\section{National Ignition Campaign Program Completion Report}

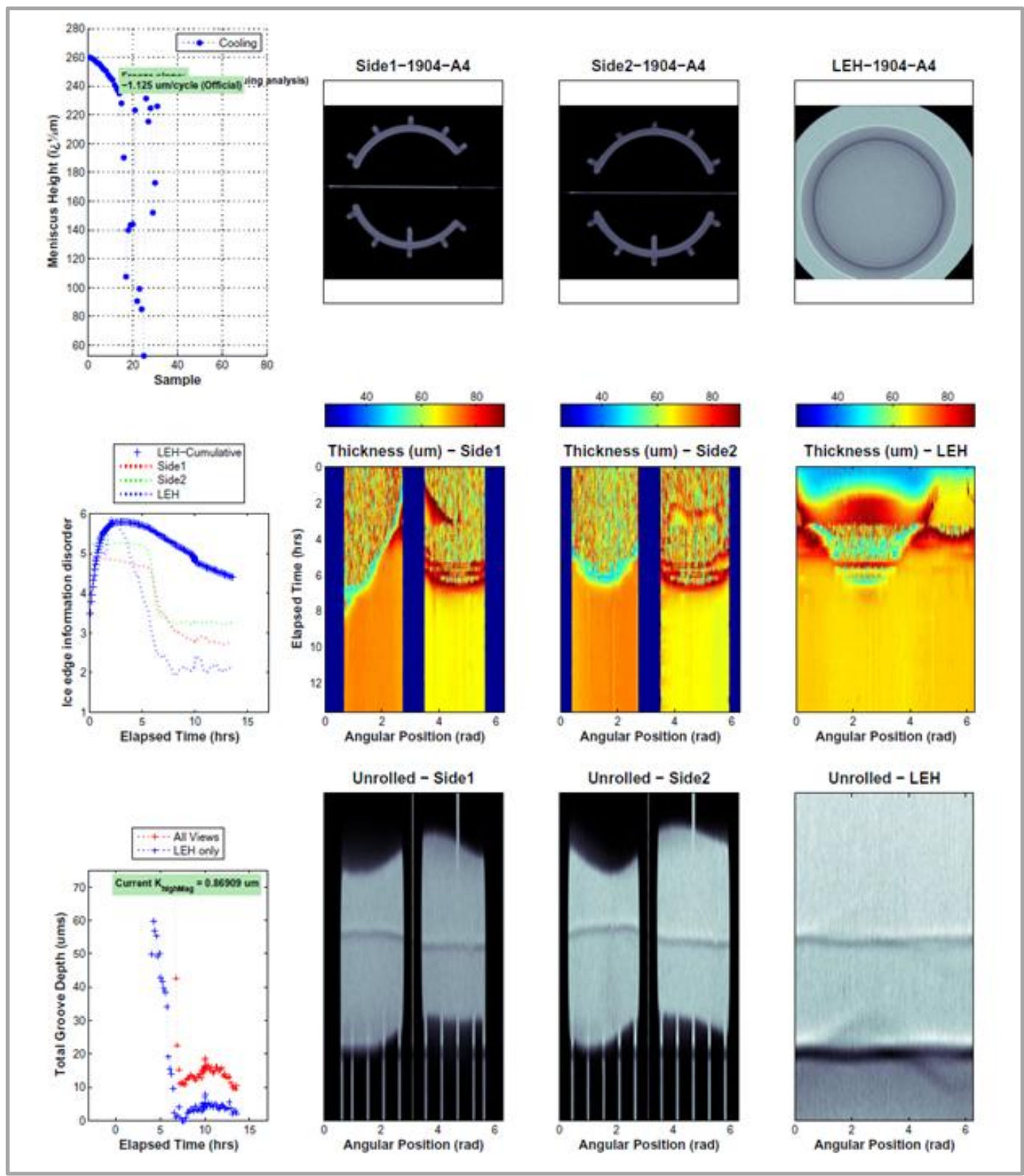

Figure 4-23. Example of the real-time dashboard used in the NIF Control Room by cryogenic system operators to track quality of a growing layer. This layer, shot on September 9, 2012, was near-ignition quality. The dashboard view shows as-grown low-mode shape errors that were subsequently shimmed out so that the layer met the requirements for the shot.

After the layer growth is complete, a second characterization technique is used to ensure that there are no hidden grooves that would fail the NIC specification for defect size. This is accomplished by moving the $\mathrm{x}$-ray tray to near its maximum height and acquiring a large number of lower-magnification LEH-only images (typically 900 images). This technique trades off resolution with contrast to ensure that no large grooves are missed. An example of this technique is shown in Figure 4-24. 


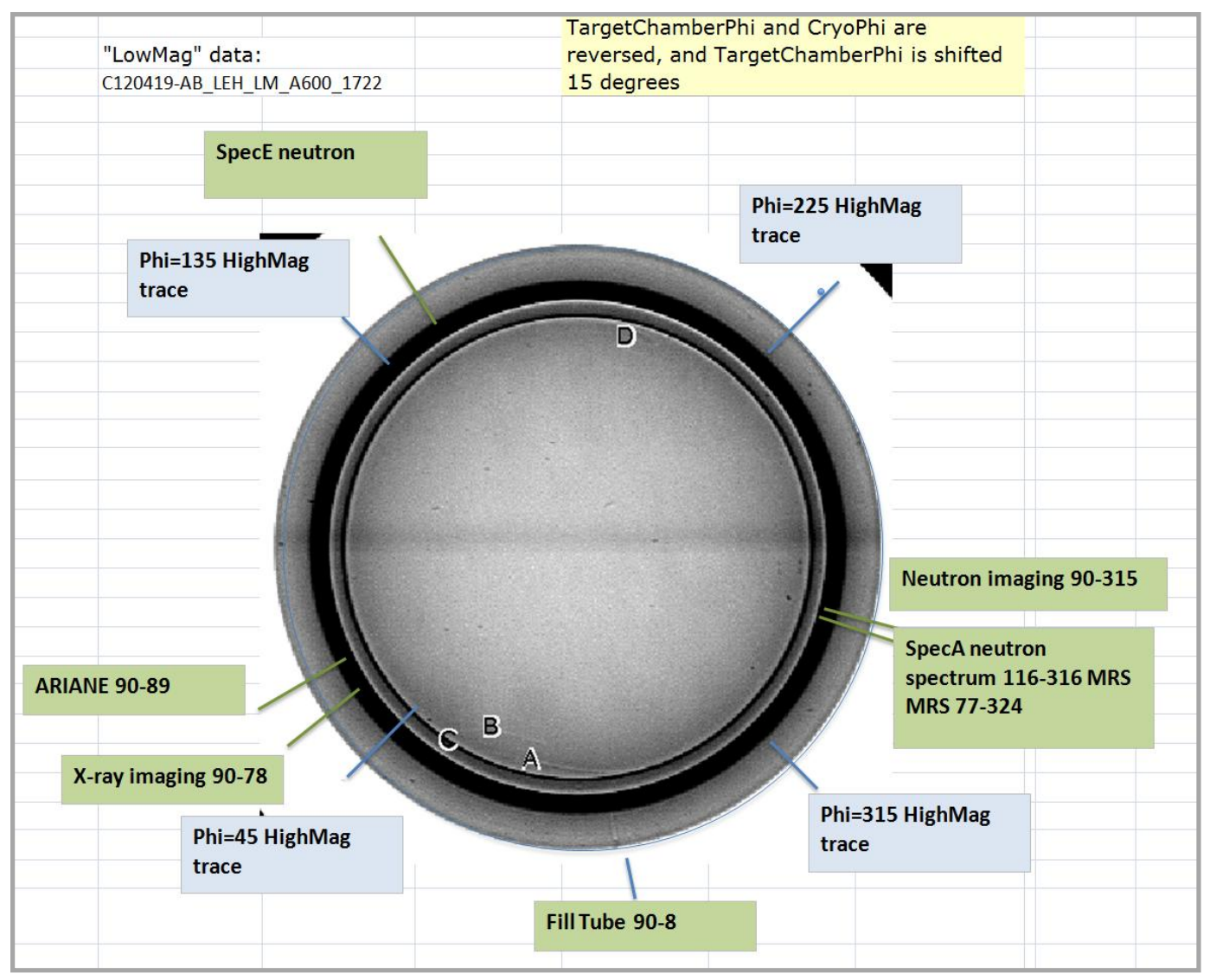

Figure 4-24. "Low-mag" image taken of a layer shot on April 22, 2012. This technique provides higher contrast at lower magnification to ensure that no defects are missed by the three-view x-ray characterization. It also provides the location of large defects, which can be related to positioning of various diagnostics around the target chamber.

\section{NIF System Demonstration}

The CTS was assembled in an off-line facility where the software control of the many sub-components was extensively tested. In March 2010, the CTS was moved and attached to the NIF target chamber and was operationally qualified in August. Later in August, a THD layering test target was installed on the ITIC in the CTS and within two days, the cryogenic team had succeeded in forming and characterizing the first $\mathrm{D}_{2}$ meniscus using contrast $\mathrm{x}$-ray imaging in three orthogonal directions using the CTS. To test layer quality at TCC during and after the alignment process and all the way through the time of the shot, on September 18, a well-characterized THD layer was taken to TCC, aligned, and then brought back to the LLCS. Subsequent re-characterization showed that layer quality did not change. This layer was then returned to TCC and a "mock" shot was executed to test the quench and shroud opening steps of the process without firing the laser. These tests were successful and demonstrated that the CTS was capable of executing a cryogenic layered target implosion on NIF.

On September 29, 2010, at 8:27 PM, NIF fired its first integrated ignition experiment using CryoTARPOS to field a cryogenic layered target at TCC. This experiment demonstrated the integration of the complex systems required for an ignition campaign. All 192 laser beams fired $1 \mathrm{MJ}$ of laser energy into the first cryogenically layered capsule. This frozen THD layer used a fuel mixture tailored to limit yield and enable the most comprehensive physics results. All systems operated successfully, and 26 target diagnostics participated in the shot. This target, and a view of the fuel layer through its LEH, is shown in Figure 4-25. 


\section{National Ignition Campaign Program Completion Report}

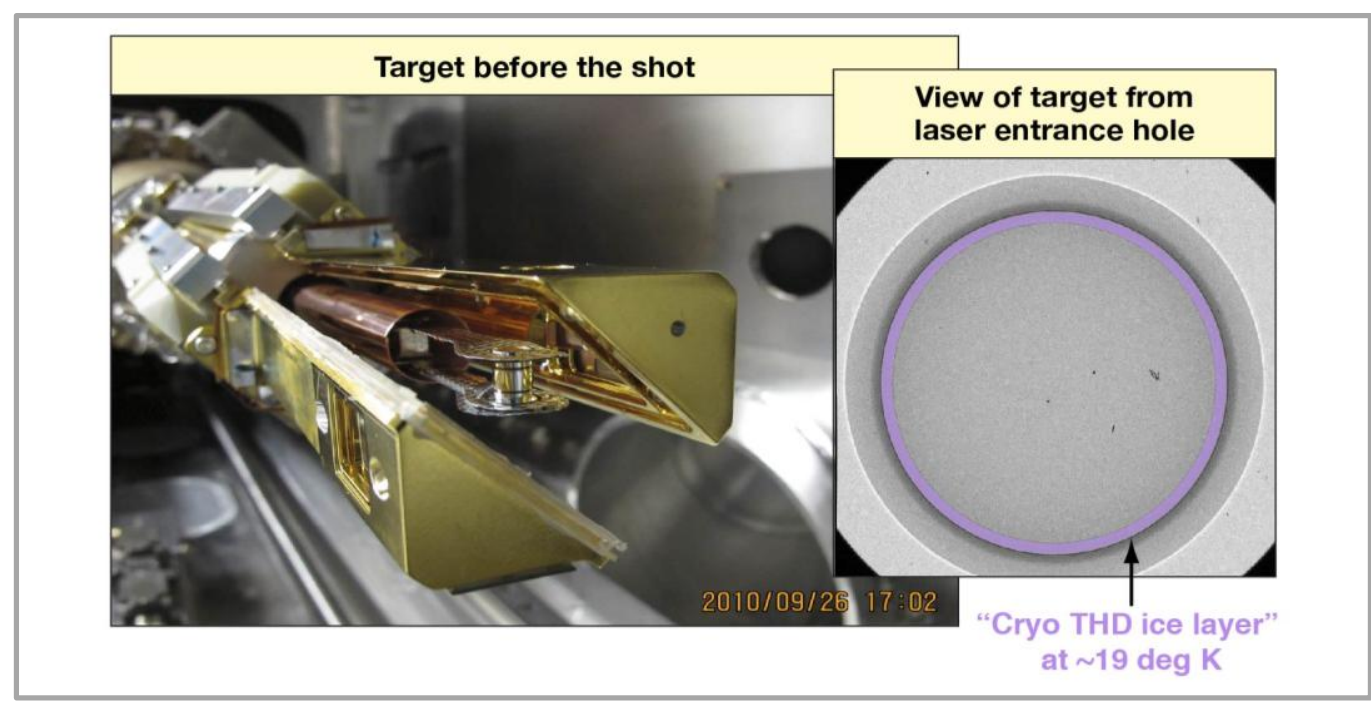

Figure 4-25. The target and capsule ice layer for the first integrated ignition experiment before the shot.

Since the demonstration of the first integrated ignition experiment in 2010, NIC has conducted nearly 40 cryogenic, layered fuel target shots on NIF. The initial process took several weeks to set up and grow a layer acceptable for a NIC shot and was heavily dependent on continuous support by engineers and scientists. Since then, the hardware, process, and layering software has evolved such that operations personnel can reliably produce a high-quality layer for a shot in four days with minimal input from the scientific staff (see Figure 4-26).

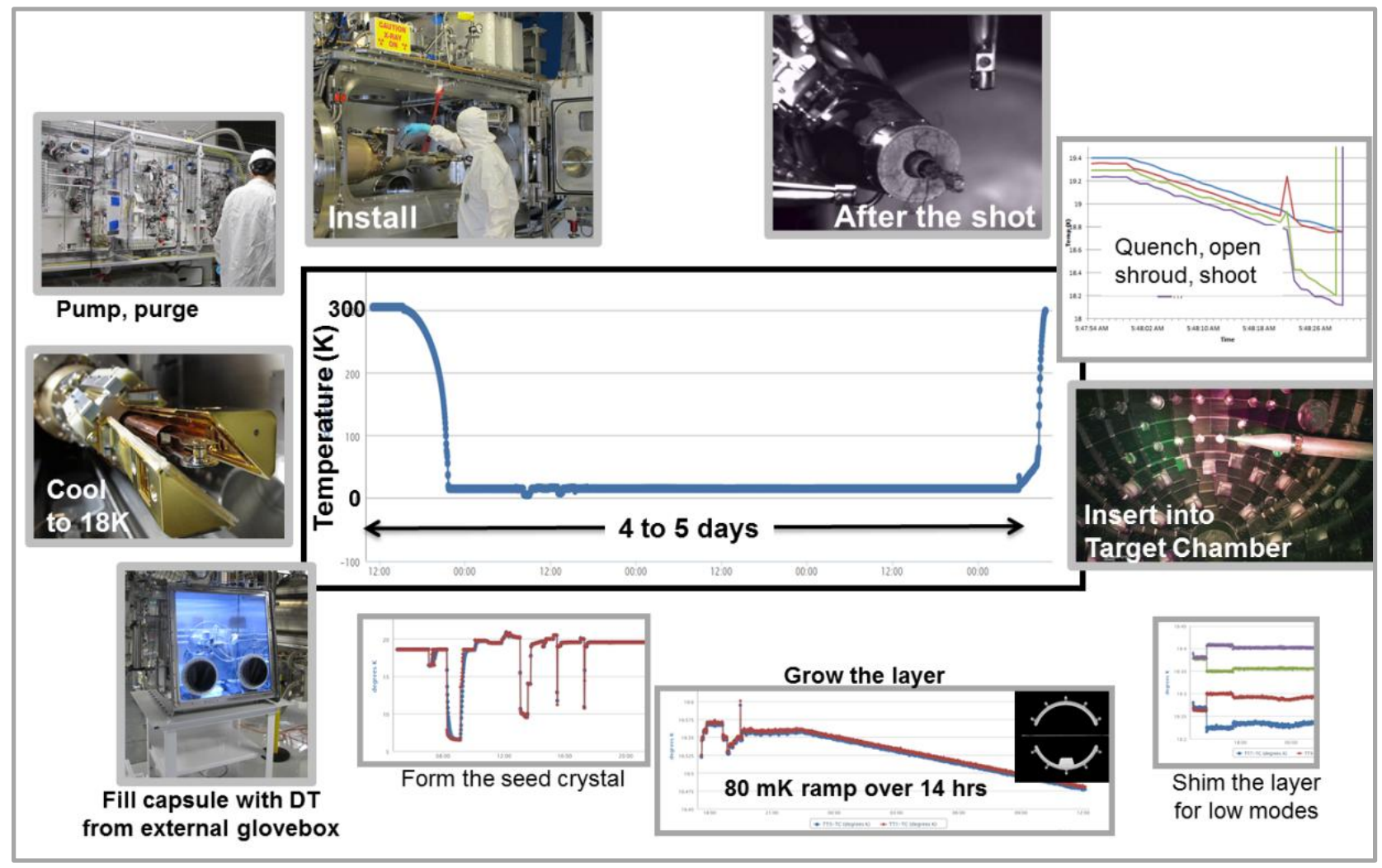

Figure 4-26. The layering process on NIF routinely grows a DT layer meeting NIC requirements in four days, allowing a layered shot as often as every four days. 


\section{National Ignition Campaign Program Completion Report}

\section{References}

1. K.A. Moreno et al. "Evolution of the Capsule Fill Tube Assembly Production Methods for the National Ignition Campaign," Fusion Sci. Technol. 59, 46 (2011).

2. Z.Z. Johal et al., "Robust Capsule and Fill Tube Assemblies for the National Ignition Campaign," Fusion Sci. Technol. 59, 331 (2011).

3. B. Lairson et al. "Laser Entrance Hole Window Burst and Pressure Deflections at Cryogenic Temperature," Fusion Sci. Technol. 59, 262 (2011).

4. E.T. Alger et al., "NIF Target Assembly Metrology Methodology and Results," Fusion Sci. Technol. 59, 269 (2011).

5. M. M. Biener et al., "Controlled Incorporation of Mid-to-High Z Transition Metals in CVD Diamond," Diamond \& Related Materials 19, 643 (2010).

6. S.J. Shin et al., "Xenon doping of glow discharge polymer by ion implantation," Applied Physics Letters 111, 096101 (2012).

7. M. Wiora et al., "Grain size dependent mechanical properties of nanocrystalline diamond films grown by hot-filament CVD," Diamond \& Related Materials 18, 927 (2009); M. Wolfer et al., "Crystallographic anisotropy of growth and etch rates of CVD diamond," Diamond \& Related Materials 18, 713 (2009).

8. M. Stadermann, S.A. Letts, and S. Bhandarkar, "Improvements to Formvar Tent Fabrication Using the Meniscus Coater," Fusion Sci. Technol. 59, 58 (2011).

9. S. Bhandarkar, T. Parham, and J. Fair, "Modeling and Experiments of Compressible Gas Flow Through Microcapillary Fill Tubes on NIF Targets," Fusion Sci. Technol. 59, 51 (2011).

10. S.A. Letts et al., "Quality Assurance and Characterization of Adhesives used for NIC Target Assembly," Fusion Sci. Technol. 59, 63 (2011).

11. R.C. Montesanti et al., "Lessons from Building Laser-Driven Fusion Ignition Targets with the Precision Robotic Assembly Machine," Fusion Sci. Technol. 59, 70 (2011).

12. A.J. Detor et al., "Stress and microstructure evolution in thick sputtered films," Acta Materialia 57, 2055 (2009); J. Biener et al., "Diamond Spheres for Inertial Confinement Fusion," Nuclear Fusion 49, 112001 (2009); L.A. Zepeda-Ruiz et al., "Understanding the Relation between Stress and Surface Morphology in Sputtered Films: Atomistic Simulations and Experiments," Appl. Phys. Lett. 95, 151910 (2009); L.A. Zepeda-Ruiz et al., "Surface Morphology Evolution During Sputter Deposition of Thin Films - Lattice Monte Carlo Simulations, " Journal of Crystal Growth 312, 1183 (2010); K.L. Sequoia et al., "Increased X-Ray Opacity of GDP Capsules from High Intensity X-Ray Exposure," Fusion Sci. Technol. 59, 35 (2011).

13. K. Youngblood et al., "Improving the Reproducibility of the Radial Argon Concentration in Beryllium Shells," Fusion Sci. Technol. 59, 126 (2011).

14. H. Huang etal., "Metrology Statistics For NIF Tuning Campaign," Fusion Sci. Technol. 59, 26 (2011).

15. A.Q.L. Nguyen et al., "Characterization of Isolated Defects for NIF Targets using PSDI with an Analysis of Shell Flipping Capability," Fusion Sci. Technol. 55, 399 (2009).

16. J.R. Fong et al., "X-Ray Absorption Spectroscopy for ICF Target Characterization," Fusion Sci. Technol. 55, 367 (2009).

17. C.R. Gibson et al., "Design of NIF Cryogenic Target System," Fusion Sci. Technol. 55 (2009).

18. E.T. Alger et al., "Experimental D-T Ice-Layering Target Assembly," Fusion Sci. Technol. 55, 269 (2009).

19. J.A. Koch et al., "Optical and X-Ray Characterization of Groove Profiles in D-T Ice Layers," Fusion Sci. Technol. 55, 244 (2009); N. Izumi et al., "Development of a Laser-Produced Plasma XRay Source for Phase-Contrast Radiography of D-T Ice Layers," Fusion Sci. Technol. 55, 253 (2009).

20. B.J. Kozioziemski et al., "Deuterium-Tritium Fuel Layer Formation for the National Ignition Facility," Fusion Sci. Technol. 59, 14 (2011); B.J. Kozioziemski et al., "Metastable polymorphs of hydrogen isotopes solidified near the triple point," Phys. Rev. B82, 012104 (2010); A.A. Chernov et al., "Single crystal growth and formation of defects in deuterium-tritium layers for inertial confinement nuclear fusion," Appl. Phys. Lett. 94, 064105 (2009); B.J. Kozioziemski et al., "Plastic deformation of solid hydrogen in fusion targets," J. Appl. Phys. 105, 093512 (2009). 


\section{I.5-TARGET DIAGNOSTICS AND EXPERIMENTAL SYSTEMS}

The primary scope of the NIC WBS Element I.5, Target Diagnostics and Experimental Systems, is provision of a core set of optical, $\mathrm{x}$-ray, neutron, and radiographic diagnostics sufficient to support experimental campaigns and measure/verify laser and target performance as an integral part of these campaigns. This WBS includes design, integration, qualification, control, and operation of diagnostics, as well as the target area systems necessary for executing experiments and the requisite off-line and on-line testing and calibration. The scope as executed also includes data analysis and archiving. Other facilities, particularly OMEGA, have been important for development and calibration of target diagnostic systems.

\section{A. Target Diagnostics Background}

Plans for NIF diagnostics began with the Nova Technical Contract in the early-1990s. At that time, the Joint Central Diagnostic Team was formed to coordinate efforts, working with their home laboratories, to develop NIF diagnostics through ICF program funding at the various NNSA laboratories. The resulting strategy called for a national effort to develop and implement a comprehensive suite of diagnostics on NIF. Moreover, it was recognized that these diagnostics would be implemented in a phased manner and when possible, multiple diagnostics would be available to measure key observables. The need for multiple complementary and redundant diagnostics was recognized as an essential requirement because no single diagnostic makes a perfect measurement. For example, thermonuclear yield is measured by three absolute and independent diagnostics.

Many diagnostics that have been implemented on NIF are adaptations of previous diagnostics developed for the nuclear test program at the Nevada Test Site (now known as the Nevada Nuclear Security Site); examples include neutron imaging (PINEX), gamma reaction history, and radiochemistry. Similarly, other diagnostics were developed for experiments on laser facilities such as Nova, OMEGA, and Janus or the $\mathrm{Z}$ pulsed power machine and then further adapted and improved for use on NIF. Although all diagnostics are integrated and operated by the NIF operations and engineering staff, the responsibility for the design, construction, initial testing and accuracy of the diagnostics systems is shared among the NIC partners. The extraordinary contributions to NIF diagnostics by NIC partners and collaborators can be found in the references at the end of this section.

The diversity of experimental requirements and finite diagnostics space has necessitated many of the

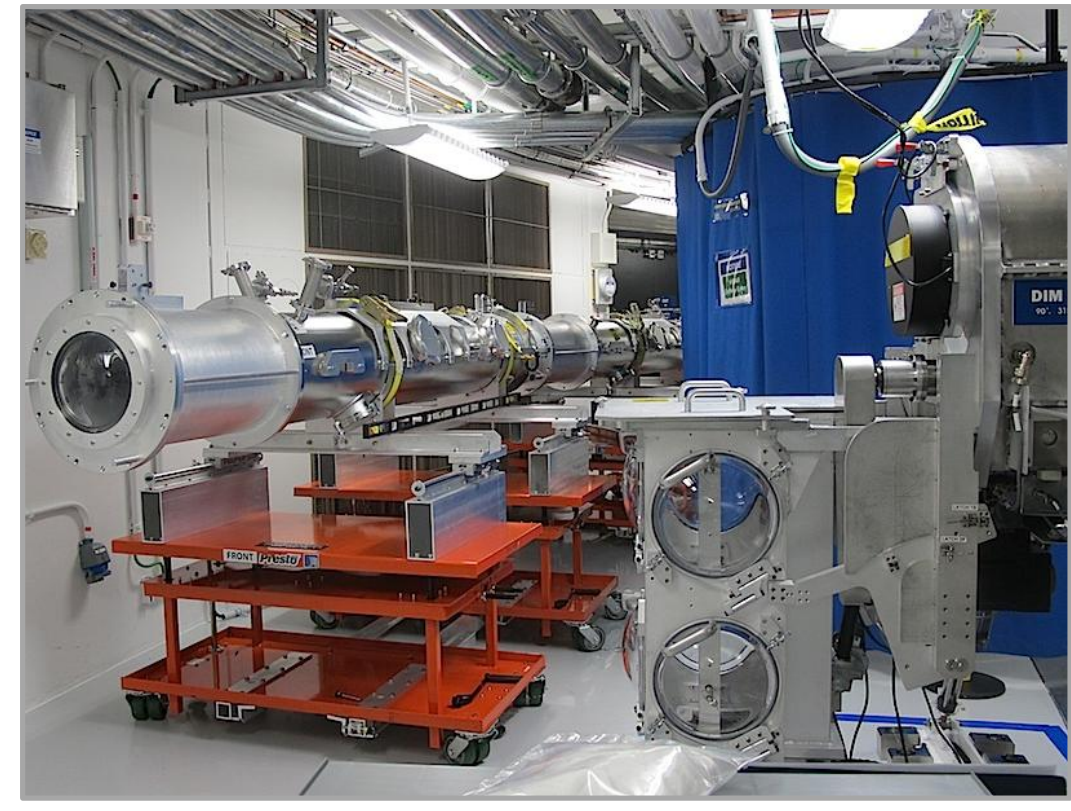

Figure 5-1. DIM Handling units and orange carts are staged for an exchange between the Neutron Imager and VISAR instruments. instruments at major ICF facilities to be removable and exchangeable. This is accomplished with vacuum-interlocked DIMs that are used to place and align instruments close to the target (see Figure 5-1). Manipulators were developed collaboratively, starting in the 1990s, and OMEGA and OMEGA Extended Performance each have six 


\section{National Ignition Campaign Program Completion Report}

Ten-Inch Manipulators (TIMs). The standardized interface on TIMs and DIMs has allowed movable DIM-based diagnostics to be easily tested at OMEGA.

\section{B. Categorization and Function of NIC Diagnostics}

NIF is now equipped with approximately 60 nuclear, optical, and x-ray diagnostics that together provide 300 channels for experimental data. More than half of the diagnostics are fielded on most shots. Operation of the set of diagnostics requires set up and control of 13,000 parameters and 1,000 control points and configuration control of thousands of diagnostic parameters. There are about 60 major data analysis algorithms. Most data is archived and is available to and searchable by qualified users (see I.2, Systems Engineering).

NIF's diagnostics can be divided into three major categories according to their principal functions:

- Diagnostics of laser absorption and hohlraum conditions, also referred to as drive diagnostics.

- Diagnostics of the shock and implosion phase, also referred to as target response/implosion diagnostics because not all experiments are implosions.

- Fuel assembly, stagnation, and heating diagnostics.

\section{Diagnostics of Laser Absorption and Hohlraum Conditions}

The NIF diagnostics that measure the laser absorption and hohlraum conditions (specifically, the radiation drive) are shown in Table 5-1.

Table 5-1. Diagnostics of laser absorption and hohlraum conditions.

\begin{tabular}{|c|c|c|c|c|}
\hline Acronym & Diagnostic & Contributors & Observable & Ref. \\
\hline $\begin{array}{l}\text { Dante1 } \\
\text { Dante2 }\end{array}$ & $\begin{array}{l}\text { Broad-band, time-resolved x-ray } \\
\text { spectrometer }\end{array}$ & LLNL & Hohlraum x-ray conditions & 1 \\
\hline EHXI & Equatorial Hard X-ray Imager & LLNL & $\begin{array}{l}\text { Beam pointing in the } \\
\text { hohlraum }\end{array}$ & \\
\hline EMP & Electromagnetic Power & LLNL & Microwave generation & \\
\hline $\begin{array}{l}\text { FABS31 } \\
\text { FABS36 } \\
\end{array}$ & $\begin{array}{l}\text { Full Aperture Backscatter } \\
\text { Station }\end{array}$ & LLNL & $\begin{array}{l}\text { Backscattered light into } \\
\text { lenses }\end{array}$ & 2 \\
\hline $\begin{array}{l}\text { FFLEX } \\
\text { FFLEX TR }\end{array}$ & Filter Fluorescer & LLNL/AWE & $\begin{array}{l}\text { Hot electron fraction and } \\
\text { temperature }\end{array}$ & 3 \\
\hline $\begin{array}{l}\text { NBI31 } \\
\text { NBI36 }\end{array}$ & Near Backscatter Imager & LLNL & $\begin{array}{l}\text { Backscattered light near } \\
\text { lenses }\end{array}$ & 2 \\
\hline $\begin{array}{l}\text { SXI-L } \\
\text { SXI-U }\end{array}$ & Static X-ray Imager & LLNL & $\begin{array}{l}\text { Laser entrance hole size } \\
\text { and beam pointing }\end{array}$ & 4,5 \\
\hline
\end{tabular}

Dante1 and Dante2: Dante1 and 2 are fixed soft x-ray power diagnostics for the upper and lower hemispheres. Each Dante has 18 different time-resolved channels; spectral ranges are controlled by the filter packs, filters, and metallic mirrors. Dante1 has five channels with mirrors, and Dante 2 has eight mirrored channels. With knowledge of the size of the LEH, Dante determines the time-dependent radiation temperature in the hohlraum from the Stefan-Boltzmann law (see Figure 5-2). 


\section{National Ignition Campaign Program Completion Report}

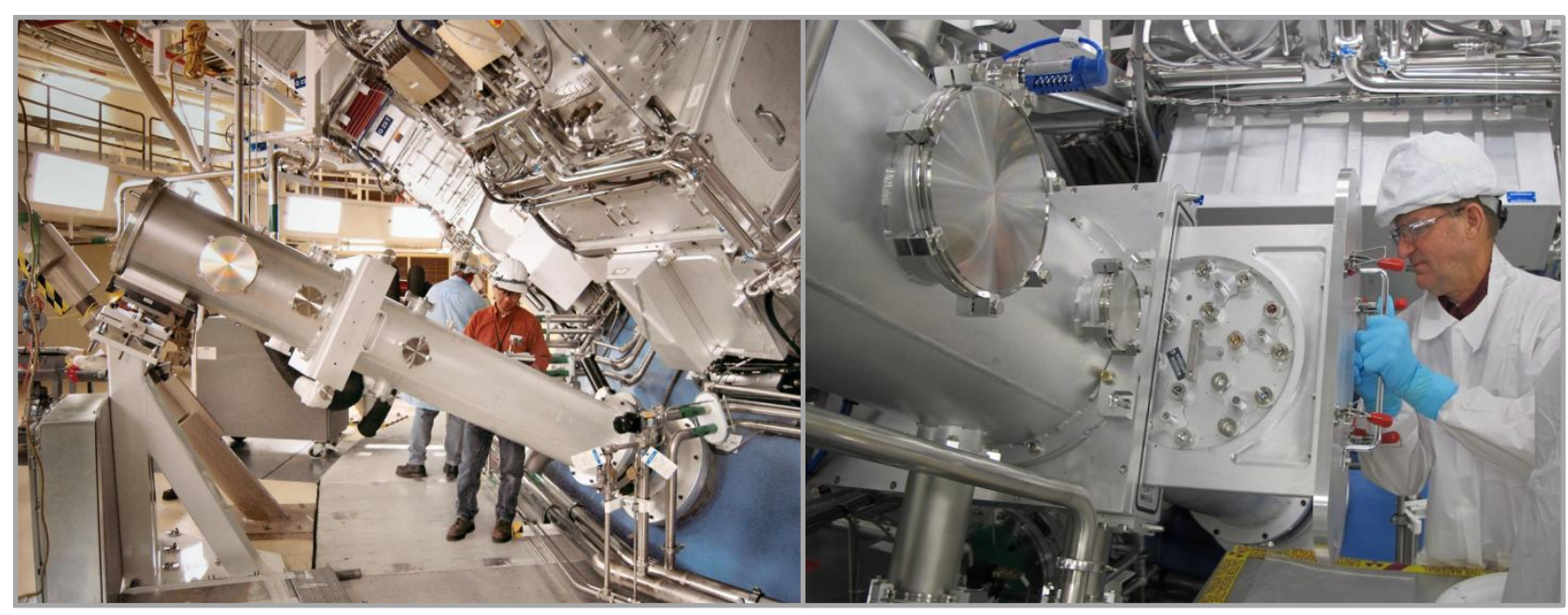

Figure 5-2. The Dante diagnostic (with a close-up of the Dante filter wheel being inserted, shown at right) is a broad-band $x$-ray spectrometer used to measure hohlraum conditions.

EHXI: The Equatorial Hard X-ray Imager is a static array of pinholes that form many low-resolution hard $(>40 \mathrm{keV}) \mathrm{x}$-ray images of the positions of the beams in the hohlraum from the $\mathrm{x}$-rays transmitted through the hohlraum walls and the TMP. The pinhole array is outside of the chamber at about $6 \mathrm{~m}$ from the target, with the image plate detector another $3 \mathrm{~m}$ further away. The low energy cutoff is set by of the $\mathrm{x}$-ray absorption in the hohlraum wall, TMP, and a thinned-out target chamber flange.

EMP: The Electromagnetic Power diagnostic measures the electromagnetic frequency spectrum in the target chamber.

FABS and NBI: On NIF, the energy that is absorbed by the target is the input laser energy minus the light that leaves the target. For coherent light sources, most of the light leaving the target is back or forward scattered by stimulated Brillouin or Raman scattering. Particularly for x-ray drive targets, the laser energy that is not absorbed comes back into the Wedge Focus Lenses (WFLs) and through the final turning mirror and is measured by the FullAperture Backscatter Systems on two representative quads of the inner and outer beams (at 30 degrees and 50 degrees). These systems are called FABS30 and FABS50, respectively. In addition, some light is scattered in the area around the WFLs and is measured by the three Near Backscatter Imager diagnostics on representative quads - an outer cone of beams at 50 degrees, and two inner cones of beams at 30 and 23.5 degrees. The NBIs consist of scatter plates (see Figure 5-6) close to the lenses, observed from outside the target chamber.

FFLEX: The Filter Fluorescer diagnostic measures the absolute hard $x$-rays energy in ten spectral bands $(10 \mathrm{keV}$ to $400 \mathrm{keV})$

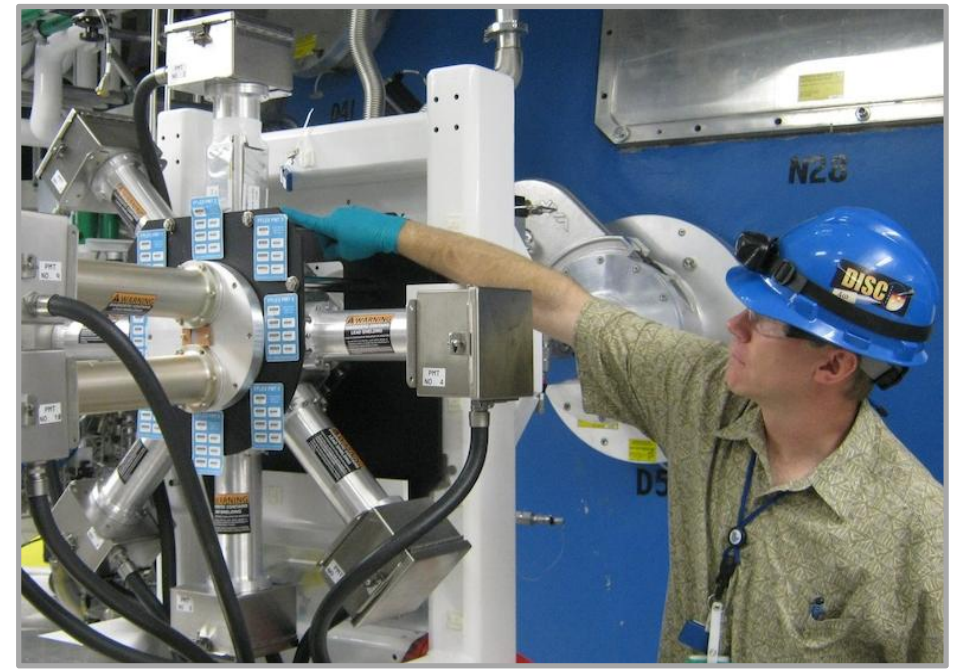

Figure 5-3. The FFLEX $x$-ray diagnostic measures electron energy during experiments by measuring the hard $x-$ ray spectrum. Shown are the eight radial direction channels, which were time integrated, and two harder (higher energy) channels, which are time resolved.

${ }^{\mathrm{z}}$ See I.4.1, Target Development and Manufacturing, for a description of TMP. 


\section{National Ignition Campaign Program Completion Report}

with time resolution on some channels. The hard x-ray spectrum determines the "temperature" and energy of hot electrons (see Figure 5-3).

SXI-U and SXI-L: Two Static X-ray Imager diagnostics (upper hemisphere and lower hemisphere), mounted on retractable positioners, perform pinhole imaging. On some channels, spectral selection is accomplished with filters and mirrors that provide low-resolution x-ray imaging at $900 \mathrm{eV}$. Other channels merely use filters, providing 3-6 keV spectral imaging. The images are recorded on either image plates or charge coupled devices (CCDs), depending on the expected neutron yield. The positions of the SXIs are chosen to view the X-rays from the inner walls of the hohlraums through the LEH. SXI was one of the original diagnostics used to assist in the verification of laser pointing during NIF commissioning. This instrument instruments can determine the time-integrated size of the LEH (taking into account closure during the laser pulse) and the positions of the laser spots focused on a planar target (by measuring the resulting $\mathrm{x}$-ray emission with respect to fiducial markings on the target).

\section{Target Response/Implosion Diagnostics}

The $\mathrm{x}$-rays produced by the interaction of the laser beams with the inside wall of a hohlraum drive shocks into the capsule within the hohlraum. As a result, the capsule is accelerated inwards, causing the fuel inside to implode and after an interval of time, stagnate at the center of the shell. The uniformity and velocities of the shocks, the shape of the hot spot at stagnation, and the interval of time when stagnation occurs are measured with the set of diagnostics shown in Table 5-2.

Table 5-2. Target Response/Implosion Diagnostics.

\begin{tabular}{|c|c|c|c|c|}
\hline Acronym & Diagnostic & Contributors & Observable & Ref. \\
\hline $\operatorname{DISC}(3 *)$ & DIM Insertable Streak Camera & LLNL/SNL & $\begin{array}{l}\text { Implosion velocity and } \\
\text { ablator thickness }\end{array}$ & $6-8$ \\
\hline GXD $(2 *)$ & Time-Gated X-ray Detector & LLNL/LANL & $\begin{array}{l}\text { Drive symmetry for low- } \\
\text { yield shots }\end{array}$ & 9 \\
\hline hGXI (2*) & Hardened X-ray Imager & LLNL/LLE & $\begin{array}{l}\text { Drive symmetry for yield } \\
<10^{15} \text { neutrons }\end{array}$ & $10-12$ \\
\hline nTOF4BT & Neutron Time-of-Flight & LLE/LLNL & Neutron bang time & 13 \\
\hline pTOF & $\begin{array}{l}\text { Particle Time-of-Flight Proton } \\
\text { Detector }\end{array}$ & MIT/LLNL & $\begin{array}{l}\text { Time of proton emission } \\
\text { from the }\left(\mathrm{D}-{ }^{3} \mathrm{He}\right) \text { reaction }\end{array}$ & 14 \\
\hline SOP & $\begin{array}{l}\text { VISAR in combination with a } \\
\text { Streaked Optical Pyrometer }\end{array}$ & LLNL & Shock break out & \\
\hline SPBT & South Pole Bang Time & LLE/LLNL & $\begin{array}{l}\text { Time of } \mathrm{x} \text {-ray emission } \\
\text { from the imploded capsule }\end{array}$ & 15 \\
\hline SPIDER & $\begin{array}{l}\text { Streaked Polar Instrumentation } \\
\text { for Diagnosing Energetic } \\
\text { Radiation }\end{array}$ & SNL/LLNL & $\begin{array}{l}\text { X-ray burn history from } \\
\text { implosion }\end{array}$ & 8,16 \\
\hline VISAR & $\begin{array}{l}\text { Velocity Interferometer System } \\
\text { for Any Reflector }\end{array}$ & LLNL/LLE & Shock velocity vs. time & 17 \\
\hline
\end{tabular}

* Number in parentheses is the number of units.

DISC: The trajectory (radius versus time or position versus time for a planar experiment) of the imploding shell is measured with DIM Insertable Streak Cameras (DISCs). These are x-ray streak cameras that have been designed to function in the harsh electromagnetic environment of the NIF target chamber. To monitor the fidelity of the streak rate and the timing, an ultraviolet $4 \omega$ fiducial (ultraviolet light) is displayed on the edge of the streak record. The x-ray streak cameras are used mainly to measure 


\section{National Ignition Campaign Program Completion Report}

the position and width of the shell as a function of time using x-ray backlighting. An earlier version of the $\mathrm{x}$-ray streak camera established the initial beam synchronicity of arrival time at TCC.

GXD and hGXI: When the imploded shell reaches the center, it stagnates, heats, and emits X-rays. The shape and time history of the x-ray emission depends in a complex way on the velocity and symmetry of the implosion and thus the intensity and symmetry of the x-ray drive. Following the evolution of the shape of the hot spot and drive symmetry by $x$-ray imaging is a technique that has been widely used in the ICF program for several decades. The instrument uses an array of pinholes projecting many images onto an electrically gated microchannel plate (MCP) microstrip, coated x-ray detector. Typically, these detectors are located about $1 \mathrm{~m}$ from TCC. Gated X-ray Detectors (GXDs) have evolved over many years to be sophisticated instruments with many control points. They use CCD detectors behind the gated MCPs and phosphors, and as a result, their use is limited to yield environments up to about $10^{13}$ neutrons. To use gated $\mathrm{x}$-ray imaging at a higher yield (up to about $10^{15}$ neutrons), hardened Gated X-ray Imager (hGXIs) are used (see Figure 5.4). In these, the CCDs are replaced by optical film, which is less sensitive to neutrons.

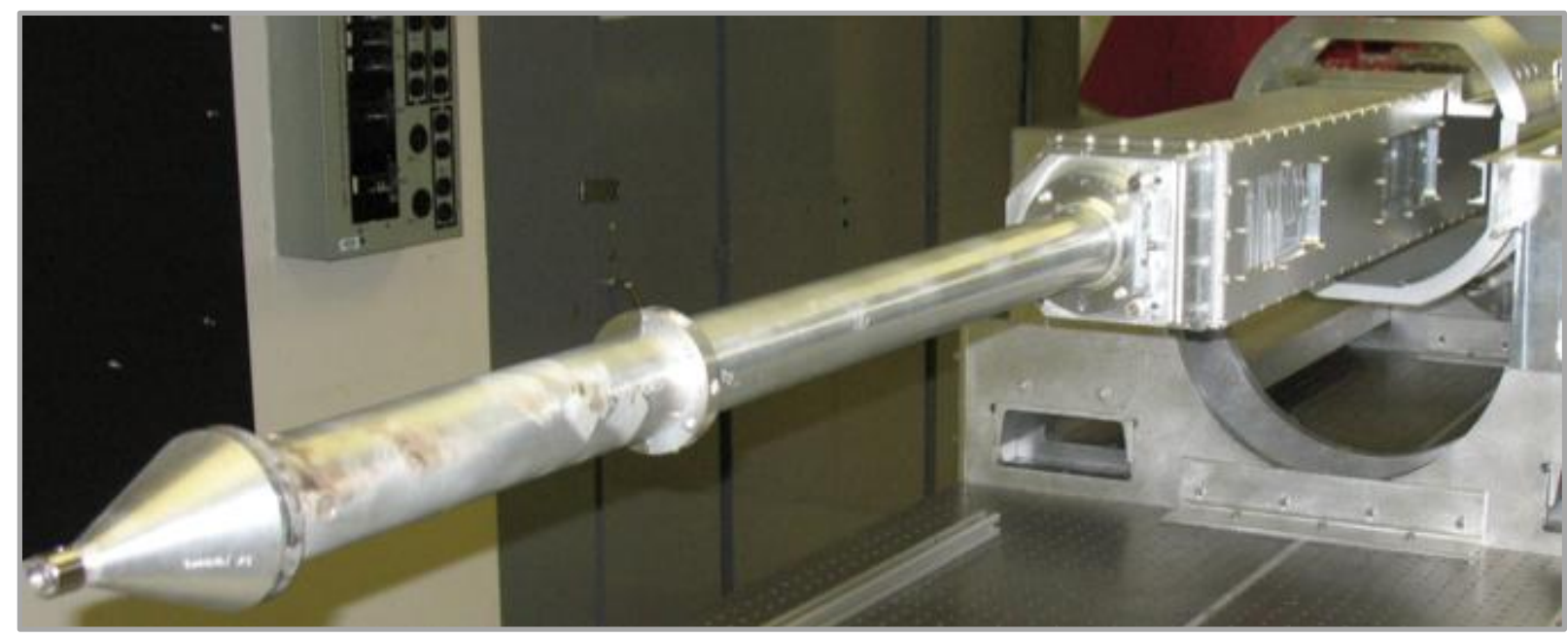

Figure 5-4. Hardened Gated X-ray Imager (hGXI) with imaging snout attached. The hGXI is the rectangular box at the back. The pinhole assembly at the front (left) end of the snout is typically located $8-10 \mathrm{~cm}$ from the target.

nTOF4BT: When the imploding target stagnates and heats, thermonuclear neutrons ${ }^{\text {aa }}$ can be emitted. A neutron detector that is relatively close to TCC can measure the time at which the burn or bang occurs or the bang time ${ }^{\mathrm{bb}}$ (BT). The Neutron Time-of-Flight-4BT (nTOF) detector in a short re-entrant well in the target chamber $4 \mathrm{~m}$ from the target measures the emission or bang time of neutrons.

pTOF: Some implosions on NIF have a gas fill of deuterium (D) and helium-3 $\left({ }^{3} \mathrm{He}\right)$ in order to produce 14.5 $\mathrm{MeV}$ protons from the $\mathrm{D}^{3} \mathrm{He}$ fusion reaction. The emission time of the protons is measured with the particle Time-of-Flight (pTOF) proton detector. The detector uses the radiation- or particle-induced conductivity in a synthetic diamond wafer detector made by the chemical vapor deposition (CVD) technique. Despite the relatively slow flight time of the protons compared to x-rays, the residual background from hohlraum x-rays is a problem for this diagnostic. Efforts are underway to reduce this background (see Figure 5-5).

\footnotetext{
${ }^{\text {aa }}$ Neutrons produced from the fusion reaction or the burning of the fusion fuel (e.g., $\mathrm{D}+\mathrm{T} \rightarrow \mathrm{n}+{ }^{4} \mathrm{He}$ ).

${ }^{b b}$ Neutron bang time is the time of the peak of the neutron burst relative to the beginning of the laser pulse.
} 


\section{National Ignition Campaign Program Completion Report}

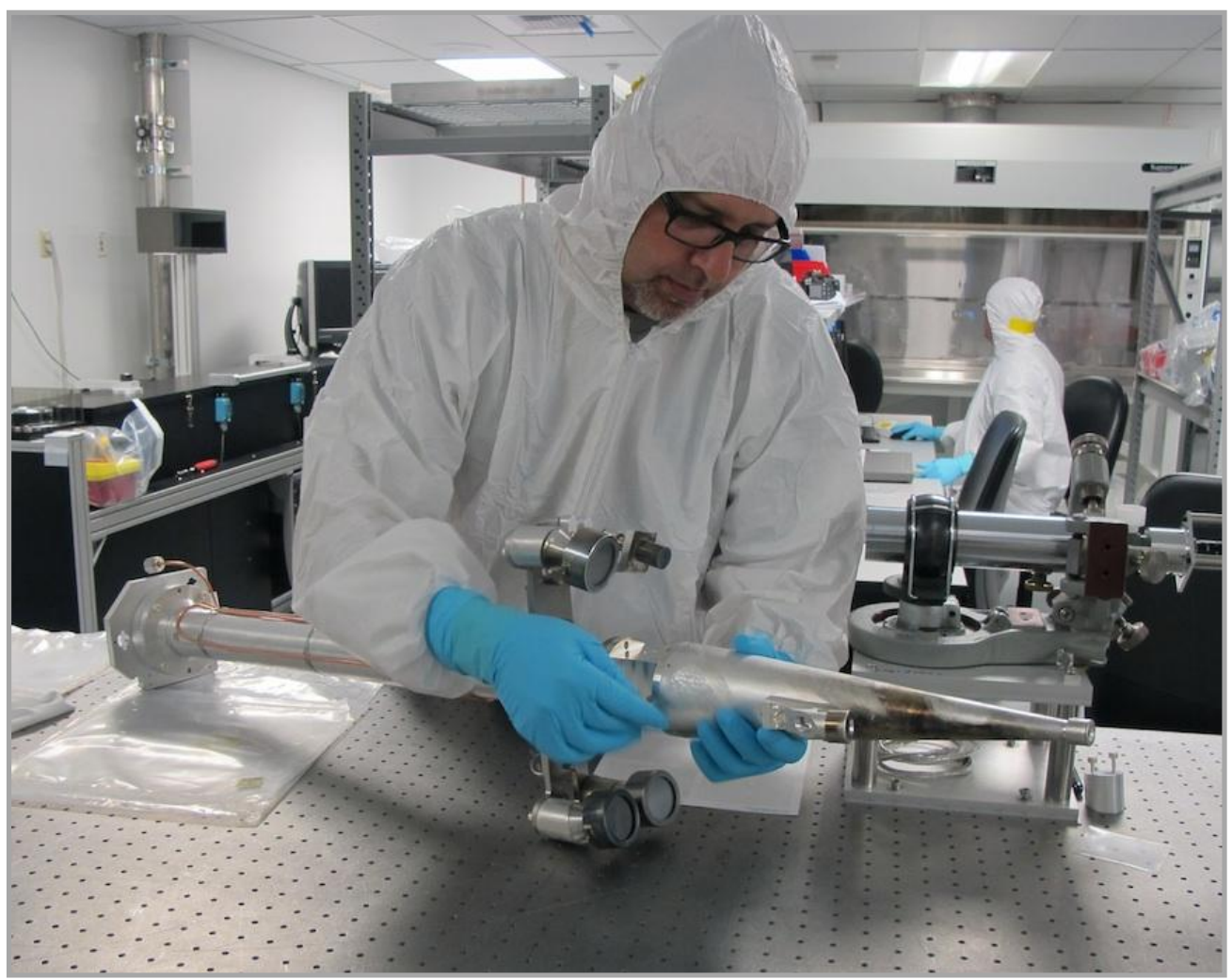

Figure 5-5. The particle Time-of-Flight (pTOF) detector is mounted on the side of the x-ray imaging snout. Here a technician assembles a snout supporting three Solid Radiochemical Collection detectors (top left and bottom) and a pTOF detector (top right) for an experiment. PTOF determines the timing of charged particles using a diamond detector that responds to neutrons and protons.

SPBT: The South Pole Bang Time (SPBT) detector measures through the lower (south) hohlraum LEH the time of peak x-ray emission (peak compression) relative to the laser pulse. This interval, which is on the order of 20 nanoseconds from the start of the laser pulse for ignition implosions, is referred to as the "x-ray bang time." The instrument has a fixed x-ray detector measuring the x-rays diffracted off a highly ordered pyroelectric graphite $\mathrm{x}$-ray crystal at a distance of about $2 \mathrm{~m}$ from TCC. The crystal is set and filtered so the SPBT is sensitive to a $1 \mathrm{keV}$ band of $\mathrm{x}$-rays at $10.8 \mathrm{keV}$. Because the signal is relayed through several tens of meters of cable to an electrical recorder, the SPBT can measure the x-ray bang time to an accuracy of only about 50 picoseconds. Therefore, the SPBT cannot accurately measure the $\mathrm{x}$-ray emission history of an implosion, the duration of which is on the order of 150 picoseconds. 


\section{National Ignition Campaign Program Completion Report}

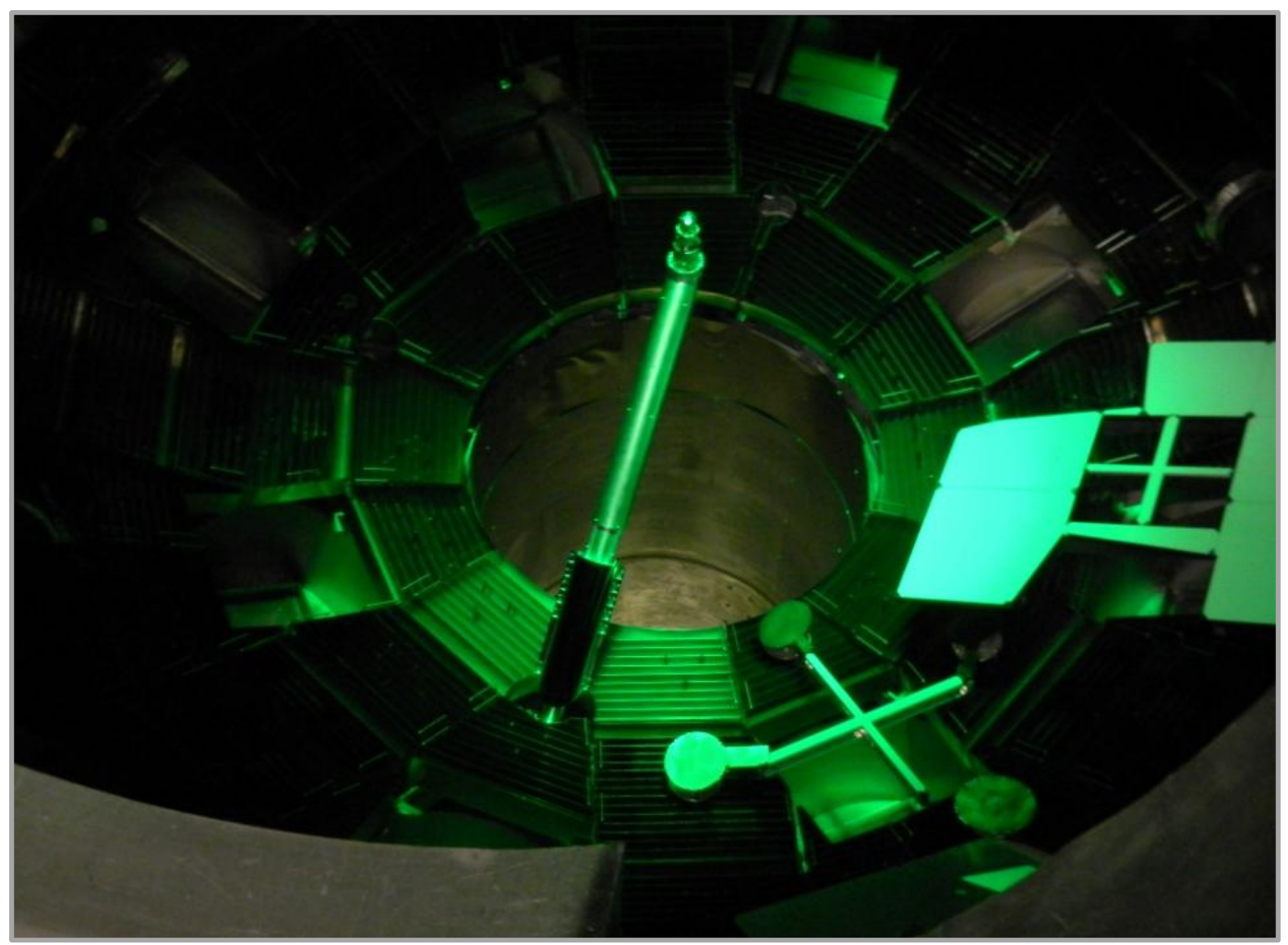

Figure 5-6. Viewing the lower hemisphere of the NIF target chamber. The South Pole Bang Time diagnostic is in the center. The scatter plates of NBI30 are on the right and the scatter cross for NBI23.5 is at the bottom right.

SPIDER: The x-ray burn history from an implosion is measured by Streaked Polar Instrumentation for Diagnosing Energetic Radiation (SPIDER). This is a fixed instrument mounted outside the chamber. It measures the $\mathrm{x}$-ray emission from an implosion at about $10 \mathrm{keV}$ through the upper LEH at a viewing angle of 7 degrees off vertical. The detector is a DISC $x$-ray streak camera, with a $4 \omega$ ultraviolet timing fiducial.

VISAR and SOP: The x-ray drive onto the capsule produces a series of shocks, which for ignition need to be delivered to the capsule at precise instants in time. The progress of the shocks through an optically transparent material (ablator or ice) is measured by the reflection of a probe laser beam to the Velocity Interferometer System for any Reflector. The break out time of an optically emitting shock is measured with the Streaked Optical Pyrometer. VISAR has been successfully used for shock timing up to the beginning of the fourth shock. A variant of VISAR technique uses a tiny mirror mounted inside a capsule that allows viewing the inside wall of the capsule at two different positions simultaneously. This is referred to as the dual-axis VISAR technique (see Figure 5-7). 


\section{National Ignition Campaign Program Completion Report}

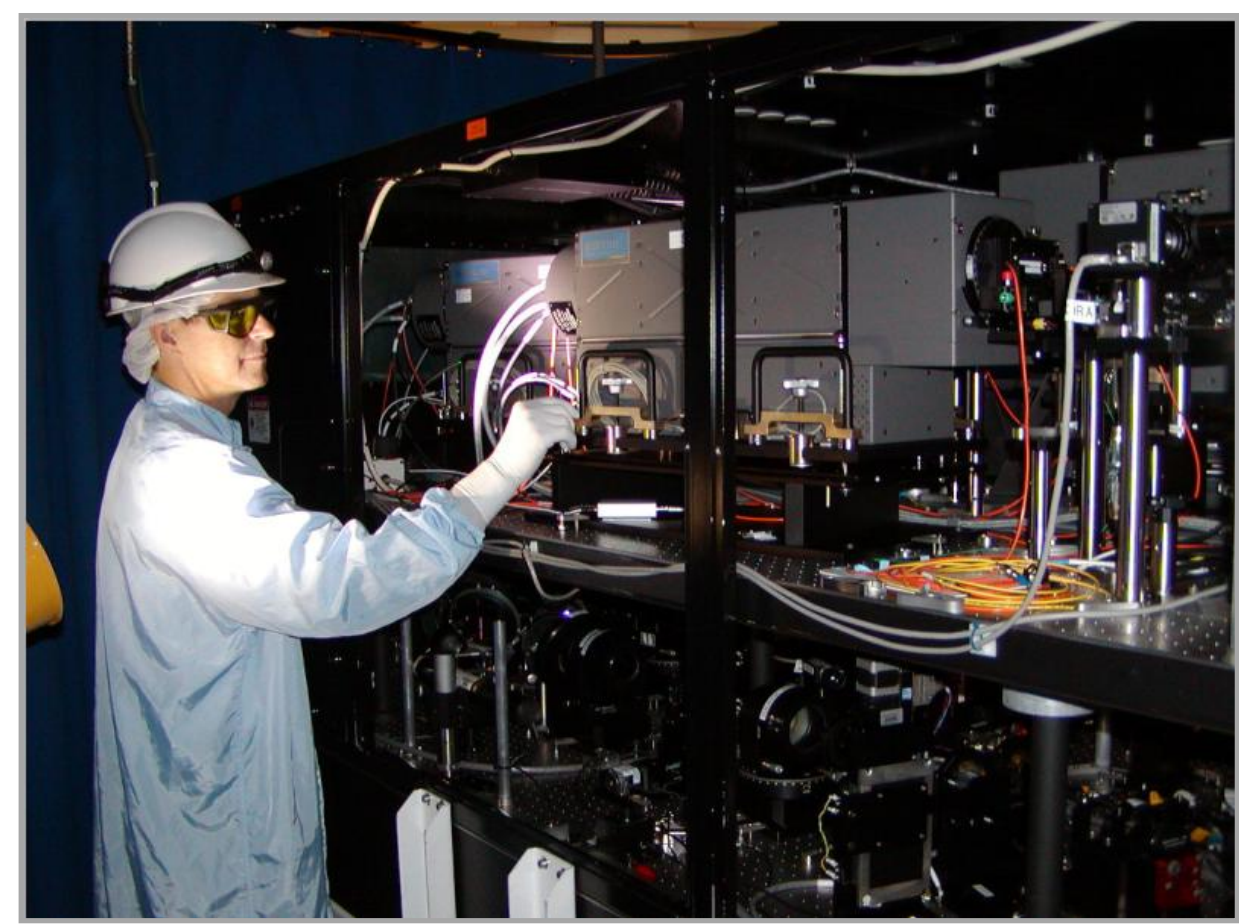

Figure 5-7. Technician is shown aligning VISAR, an interferometer used to measure shock timing.

\section{Assembly, Stagnation, and Heating Diagnostics}

As the imploding fuel and remaining ablator approach the center of the capsule, they cause the pressure and temperature of the material at the center to rise forming a hot spot. This increase in pressure and temperature leads to deceleration and stagnation of the implosion. The hot spot causes thermonuclear fusion reactions that produce high-energy neutrons, gammas, and $\alpha$-particles. NIF has a set of diagnostics to measure this phase of the implosion. The main measurement attributes of this phase are categorized as being associated with the hot spot (yield, size, burn history, and temperature), the cold compressed fuel and its areal density, and mix. The suite of fuel assembly, stagnation, and heating diagnostics are shown in Tables 5-3 through 5-5.

\section{a. Diagnostics of the Hot Spot}

The neutron yield is a primary metric of the properties of the hot spot. For DT, yield is measured by three independent absolute diagnostics, namely two types of Neutron Activation Detectors (NADs) and the Magnetic Recoil Spectrometer (MRS). These techniques are absolute in the sense that they depend on the known geometry and two different nuclear activation cross sections (copper and zirconium) and the neutron-proton cross section for MRS. The levels of accuracy for all techniques are less than 10\% and are only weakly dependent on yield for yields $>10^{13}$ neutrons. The fact that usually the three independent techniques agree adds confidence to estimates of accuracy. A more rapid diagnostic for neutron yield is provided by the nTOF detectors, but these are calibrated against the three absolute detectors. For deuterium (DD) and $\mathrm{D}^{3}{ }^{3} \mathrm{He}$ gas fills, the lower energy $2.4 \mathrm{MeV}$ neutron yield is measured by neutron activation of an indium foil and/or absolute track counting. 


\section{National Ignition Campaign Program Completion Report}

Table 5-3. Diagnostics of the hot spot.

\begin{tabular}{|c|c|c|c|c|}
\hline Acronym & Diagnostic & Contributors & Observable & Ref. \\
\hline ARIANE & $\begin{array}{l}\text { Active Readout in a } \\
\text { Neutron Environment } \\
\text { (Gated x-ray imager) }\end{array}$ & LLNL & $\begin{array}{l}\text { X-ray hot spot size and } \\
\text { shape for yields }<10^{16} \\
\text { neutrons }\end{array}$ & 18 \\
\hline DIXI & $\begin{array}{l}\text { Dilation Imager for X- } \\
\text { rays at Ignition }\end{array}$ & GA/LLNL & $\begin{array}{l}\text { X-ray hot spot size and } \\
\text { shape with an } \mathrm{x} \text {-ray } \\
\text { shutter time } \sim 10 \mathrm{ps}\end{array}$ & 19 \\
\hline GRH & $\begin{array}{l}\text { Time and spectrally- } \\
\text { resolved Gamma } \\
\text { Reaction History }\end{array}$ & LANL/LLNL & $\begin{array}{l}\text { Gamma spectrum and } \\
\text { time history }\end{array}$ & 20,21 \\
\hline $\mathrm{NAD}-\mathrm{Cu}$ & $\begin{array}{l}\text { Neutron Activation } \\
\text { Detector }\end{array}$ & SNL/LLNL & $\begin{array}{l}\text { Unscattered neutron } \\
\text { yield from a DT-filled } \\
\text { capsule }\end{array}$ & 22 \\
\hline $\begin{array}{l}\text { NAD_-DIM } \\
\text { indium (In) }\end{array}$ & $\begin{array}{l}\text { Neutron Activation } \\
\text { Detector (DIM mounted) }\end{array}$ & LLNL & $\begin{array}{l}\text { Unscattered neutron } \\
\text { yield from a DD-filled } \\
\text { capsule }\end{array}$ & \\
\hline $\mathrm{NAD}-\mathrm{Zr}$ in well & $\begin{array}{l}\text { Neutron Activation } \\
\text { Detector (well mounted) }\end{array}$ & LLNL & $\begin{array}{l}\text { Unscattered neutron } \\
\text { yield from a DT-filled } \\
\text { capsule }\end{array}$ & 23 \\
\hline NIS & Neutron Imaging System & LANL/LLNL & $\begin{array}{l}\text { Hot spot size and fuel } \\
\text { asymmetry }\end{array}$ & 24 \\
\hline nITOF & $\begin{array}{l}\text { Neutron Imaging Time- } \\
\text { of-Flight }\end{array}$ & LLE/LANL/LLNL & Ion temperature & 24 \\
\hline nTOF20 IgnHi & Neutron Time-of-Flight & LLE/LANL/LLNL & Ion temperature & 25 \\
\hline
\end{tabular}

ARIANE: The Active Readout in a Neutron Environment gated $\mathrm{x}$ ray imaging detector measures the X-ray hot spot size and shape up to a yield of $\sim 10^{16}$. ARIANE uses the gated MCP technology described for GXD and hGXI. To operate in a higher neutron yield regime, the detector is moved further away, to a location just outside of the target chamber. A plan is in place to use a mirrored version of ARIANE for experiments for operation with yields in excess of $10^{16}$ neutrons (see Figure 5-8).

DIXI: The Dilation X-ray Imager drifts and time dilates a photoelectron image of an implosion. The time dilation is caused by ramping the extraction field of the

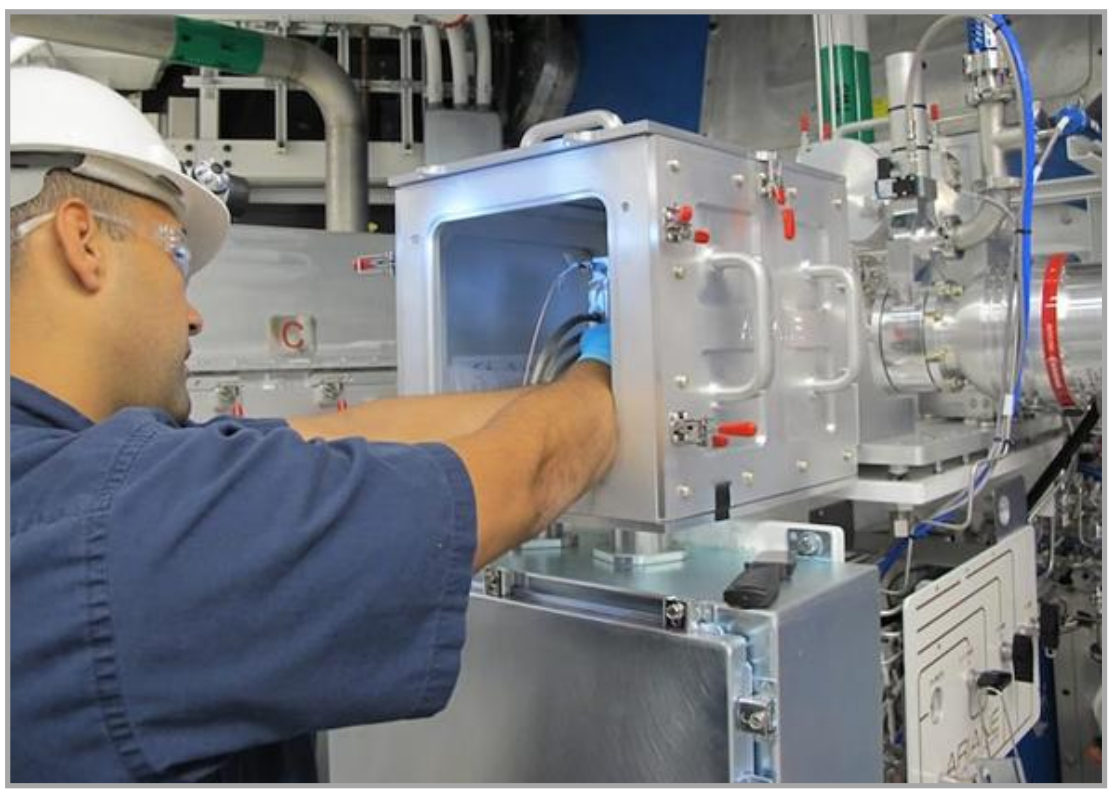

Figure 5-8. A technician installs a film pack in the ARIANE timeresolved $x$-ray imager for an experiment. ARIANE uses a CCD detector to electronically capture and record $x$-ray data for higheryield experiments. 


\section{National Ignition Campaign Program Completion Report}

photo-electron image in a magnetic field. A simple analogy is the ways cars spread out as they accelerate after stopping at a traffic light. The time dilation allows time resolution to better than $10 \mathrm{ps}$. This kind of time resolution is necessary because as the yield increases, the duration of $\mathrm{x}$-ray emission should become less than $100 \mathrm{ps}$.

GRH: The Gamma Reaction History diagnostic measures the spectrum and time history of the emission of gamma rays produced from the DT reaction and from neutrons interacting with matter. GRH is set up with four spectral channels (typically $2.9,5,8$, and 10 $\mathrm{MeV})$. In each GRH channel, gammas interact with a foil to produce Compton electrons, which recoil into a gas-filled cell. If the velocity of the Compton electrons exceeds the local speed of light as determined by the type and pressure of the gas in the cell, they will generate broad-band Cerenkov light (from 250 to 700 $\mathrm{nm})$. For each channel, Cerenkov light is relayed to a high-speed detector using an off-axis parabolic mirror. This design incorporates a fixed time delay of $4.26 \mathrm{~ns}$ that allows the detector to recover from prompt radiation due to laser-plasma interactions from the target (see Figure 5-9).

NAD_Cu: One NAD measures the unscattered neutron yield from DT-filled capsules by activating a copper foil in a neutron line of sight in the neutron alcove. Copper (and zirconium, discussed below) is an ideal energy threshold activation detector for $14 \mathrm{MeV}$ neutrons. Any scattering by the target, material in the chamber, and most importantly the chamber itself, will down-shift the energy of the neutrons sufficiently that they will not cause activation; thus only the unscattered neutron yield is measured. After the shot, the foil is removed and the activation level is determined using standard nuclear coincidence counting techniques. Because of the rapid decay rate of the activated copper (one half-life is 9.7 minutes), the foil must be quickly removed and counted in a nearby detector system (see Figure 5-10).

NAD_DIM: For the $2.45 \mathrm{MeV}$ neutrons produced in the DD fusion reaction, indium is used as the activation detector. Because indium is not a threshold detector for activation by $2.45 \mathrm{MeV}$ neutrons, the sample needs to be close to the TCC to decrease the fractional contribution of scattering from the chamber. Consequently, the samples are mounted on DIMs, typically on the side of gated $\mathrm{x}$-ray detectors also mounted in DIMs. The half-life of the indium activation product $-4.5 \mathrm{hr}$ - is comparable to the normal operational extraction time of a few hours; therefore, the comprehensive LLNL radiochemistry counting facilities in B151 can be used.

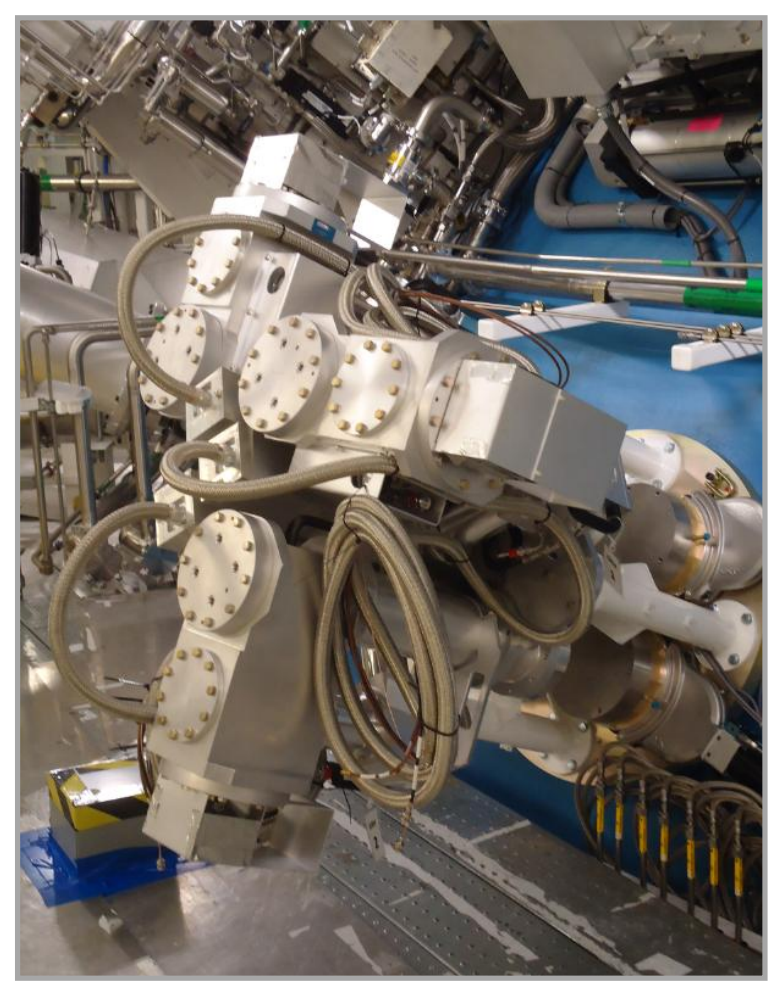

Figure 5-9. The Gamma Reaction History diagnostic is used to measure the time history gamma-ray emission. The four Cerenkov detectors are shown on the outside of the chamber.

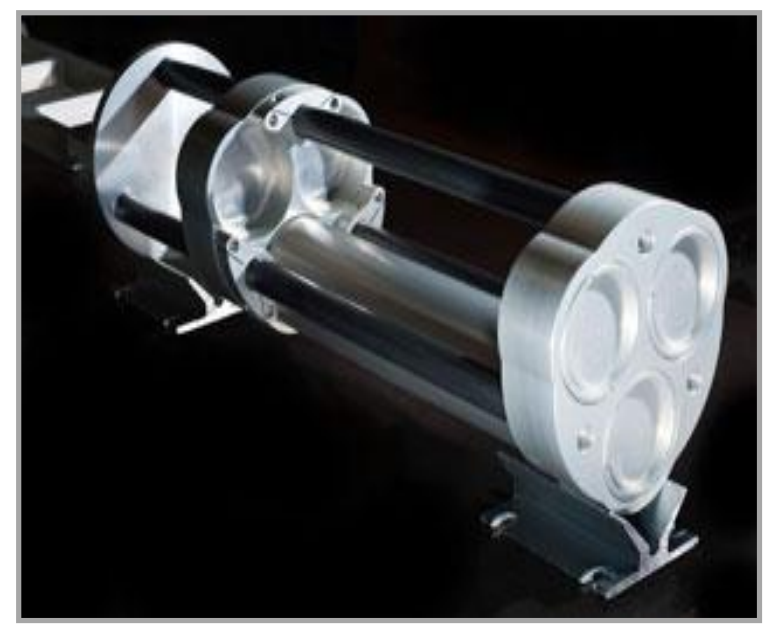

Figure 5-10. The Neutron Activation Detector in a well is designed to hold up to three zirconium samples at the front or back to measure unscattered 14.1 MeV DT neutrons. 


\section{National Ignition Campaign Program Completion Report}

NAD Well-Zr: The NAD in a well uses activation of a zirconium sample inserted into a short re-entrant well on the target chamber. Zirconium, like copper, is a threshold detector. Since the half-life of the zirconium activation product is three days, the counting facilities in the basement of building B151 can be used.

NIS: The Neutron Imaging System (NIS) creates static neutron images of the primary $(14.1 \mathrm{MeV})$ unscattered neutrons and the downscattered $(6-12 \mathrm{MeV})$ neutrons from a burning DT capsule. Imaging is by a neutron pinhole array, but because of the large range of $14 \mathrm{MeV}$ neutrons, the pinholes are precisionshaped tunnels in thick tungsten (see Figure 5-11). The neutron pinhole array is held in place by the DIM 90-315 manipulator. The detector is a scintillator but because of the nearly mm range of the knock-on protons, a high magnification is required ( 100x) to achieve 10-20 micron source resolution. The hot spot size and fuel asymmetry are determined from the image of the primary neutrons, and the cold fuel areal density is inferred from the DSR. ${ }^{\mathrm{cc}}$

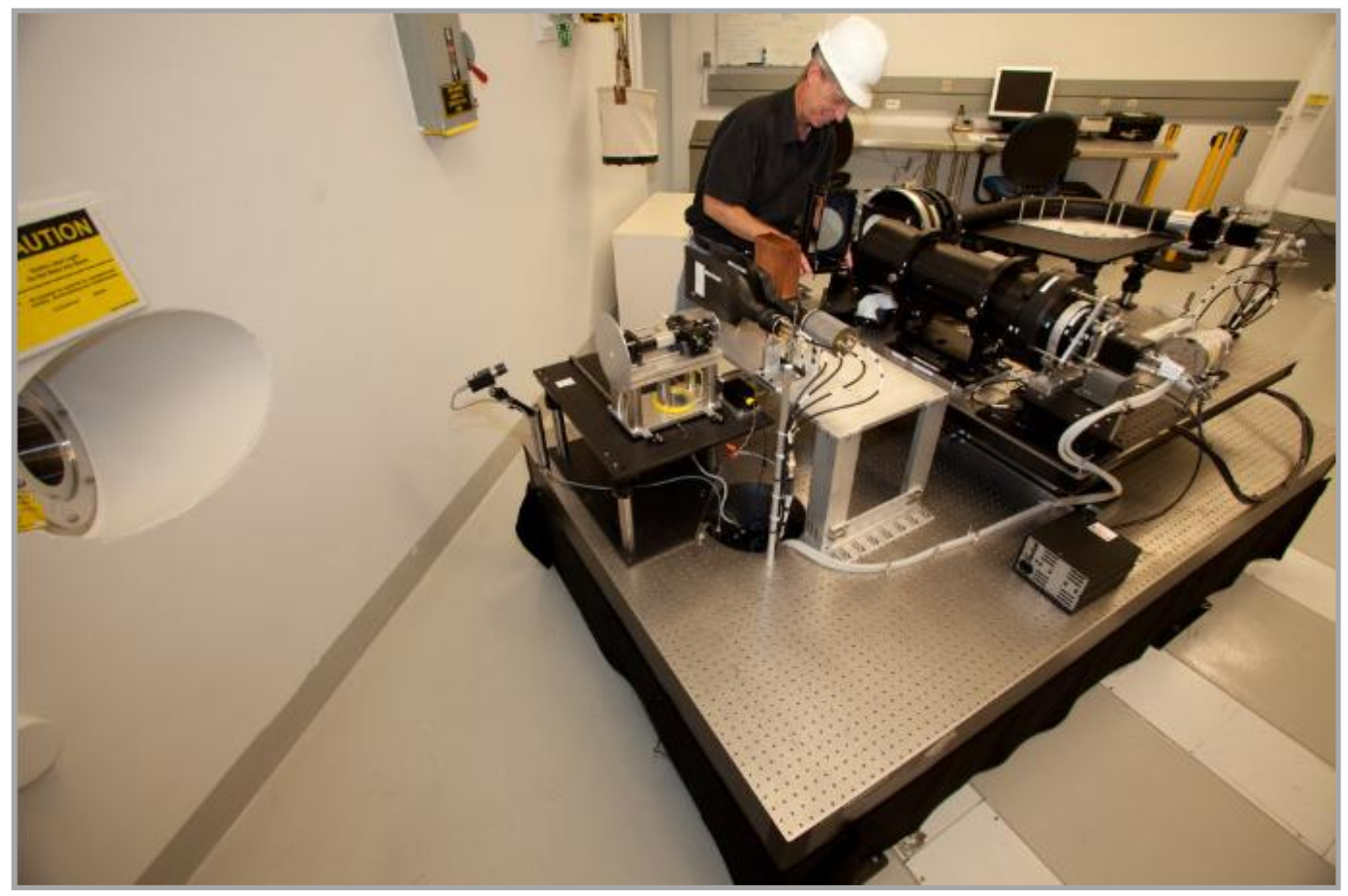

Figure 5-11. The detector end of the Neutron Imaging System.

nTOF20IgHi and nITOF: These are two separate nTOF detectors whose main function is to measure ion temperature of the hot spot from the thermal broadening of the neutrons. The nTOF20IgHi is a CVDbased synthetic diamond detector located in the neutron alcove about $20 \mathrm{~m}$ from TCC. The Neutron Imaging Time-of-Flight diagnostic (nITOF) is located around the NIS system about $28 \mathrm{~m}$ from TCC.

${ }^{\mathrm{cc}}$ Downscattered ratio $(\mathrm{DSR})=$ Number of primary neutrons $\left(\mathrm{E}_{\mathrm{n}} \sim 12-16 \mathrm{MeV}\right)$ divided by the number of downscattered neutrons $\left(\mathrm{E}_{\mathrm{n}} \sim 6-10 \mathrm{MeV}\right)$. 


\section{National Ignition Campaign Program Completion Report}

\section{b. Diagnostics of Areal Density}

Table 5-4. Diagnostics of Areal Density

\begin{tabular}{|l|l|l|l|l|}
\hline Acronym & Diagnostic & Contributors & Observable & Ref. \\
\hline CR & Compton Radiography & LLNL & Areal density & 26 \\
\hline MRS & $\begin{array}{l}\text { Magnetic Recoil } \\
\text { Spectrometer }\end{array}$ & MIT/LLE/LLNL & Areal density & $27-30$ \\
\hline $\begin{array}{l}\text { nTOF_-Flange } \\
\text { nTOF20 SPEC-E }\end{array}$ & $\begin{array}{l}\text { Detector (Flange } \\
\text { Mounted) }\end{array}$ & LLNL & Areal density anisotropy & 23 \\
\hline RAGS & $\begin{array}{l}\text { Radiochemical Analysis } \\
\text { of Gaseous Samples }\end{array}$ & LLNL & Areal density & 13 \\
\hline SRC & $\begin{array}{l}\text { Solid Radiochemical } \\
\text { Collection Diagnostic }\end{array}$ & LLNL/LANL & Areal density & 31 \\
\hline WRF & Wedged Range Filter & MIT/LLNL & $\begin{array}{l}\text { Plastic areal density for } \\
\text { deuterium and helium-3 }\end{array}$ & 33 \\
\hline
\end{tabular}

CR: ARC will be used for time-resolved radiographic imaging of the dense cold fuel surrounding the hot spot by Compton Radiography. CR is a measurement technique based on point-projection radiography at photon energies from 60 to $200 \mathrm{keV}$ where the Compton effect is the dominant contributor to the $\mathrm{x}$-ray "opacity" (of course, photons are not absorbed but scattered) of the fuel. Until ARC is available at NIF, CR with reduced resolution of about 30 microns is being performed using two focused $3 \omega$ quads of NIF. The detector is a hardened, gated $\mathrm{x}$-ray detector.

MRS: The MRS is a fixed location neutron spectrometer that provides the most accurate measurement of the compressed fuel areal density (see Figure 5-12). Neutrons interact with a plastic foil (sometimes deuterated) held $30 \mathrm{~cm}$ from the target, producing knock-on protons or deuterons. These charged particles are then energy dispersed by their momentum in a magnetic field and focused on an array of solid plastic film track detectors (CR-39) located at the focal point of the spectrometer. After a shot, the film is removed and etched and the neutron spectrum (neutrons as a function of their energy) and yield (total number of neutrons) are determined by the location and number of tracks on the detectors. The number of neutrons downscattered in energy by the compressed DT is measured by the neutron spectrum. In addition, MRS records NIF's absolute yield and ion temperature (although ion temperature is measured with lower resolution than the nTOF, depending on the thickness of the plastic foil). 


\section{National Ignition Campaign Program Completion Report}

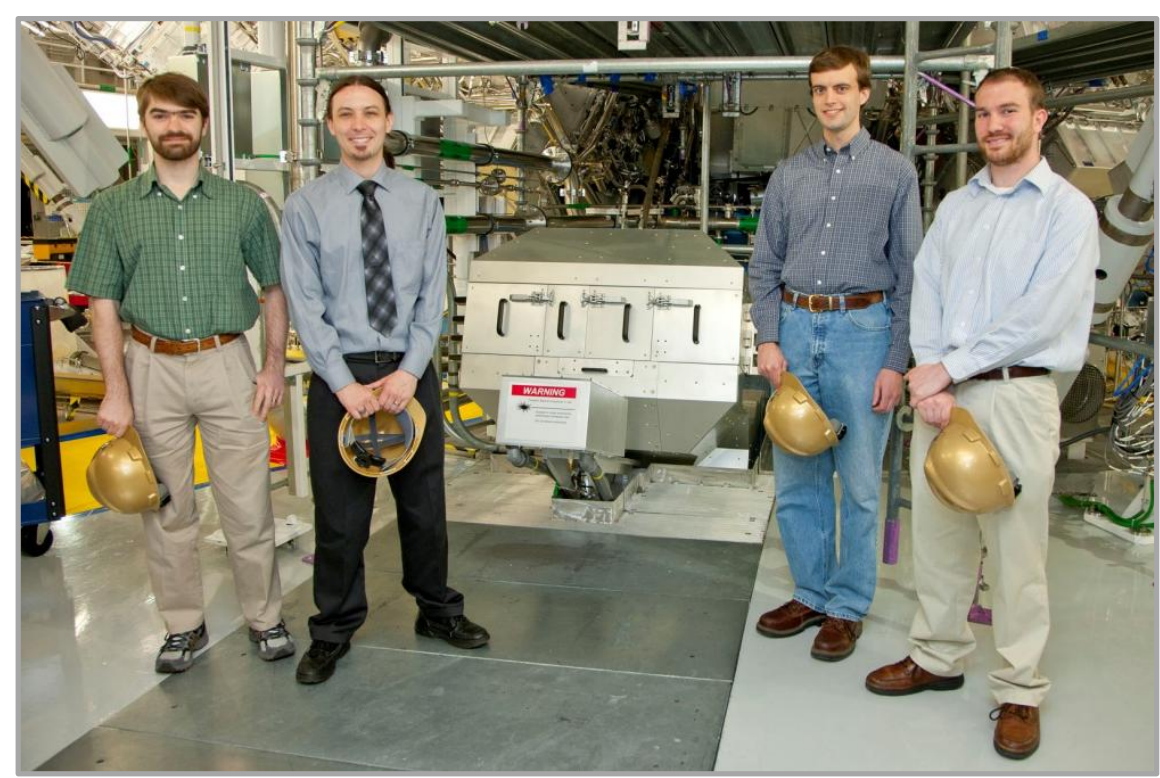

Figure 5-12. The Magnetic Recoil Spectrometer is shown with four students from MIT who were essential in implementing the instrument and the CR-39 detectors.

NADS Flange-Zr: The flange NAD system uses a set of up to 16 zirconium activation samples strategically mounted on the outside of the flanges of the target chamber. The 3-day half-life of the zirconium activation products allows the samples to be counted in B151. Most of the $14 \mathrm{MeV}$ neutrons are not scattered by the flanges and so the suite of zirconium NADs measures the anisotropy of neutron yield from the target. If the angular distribution is not isotropic (equal in all directions), a variation in yield as a function of direction indicates a variation or asymmetry in the fuel areal density. Accurately measuring the anisotropy in the neutron yield from an implosion requires an accurate cross-calibration using low areal density implosions because the compressed fuel areal density only downscatters about $20 \%$ of the $14 \mathrm{MeV}$ neutrons, and variations in that $20 \%$ are needed to measure accurately.

\section{nTOF20 SPEC-A and nTOF20 SPEC-E:}

There are two nTOFs located at a distance of approximately $20 \mathrm{~m}$ from TCC in the alcove (SPEC-A) and at the equator (SPEC-E) that are well screened against neutrons scattered off of the TARPOS and DIM and from the target chamber walls. Consequently, these diagnostics have sufficiently low backgrounds to measure neutrons downscattered by the fuel. These instruments are used to measure neutron yield, ion temperature, and areal density (see Figure 5-13).

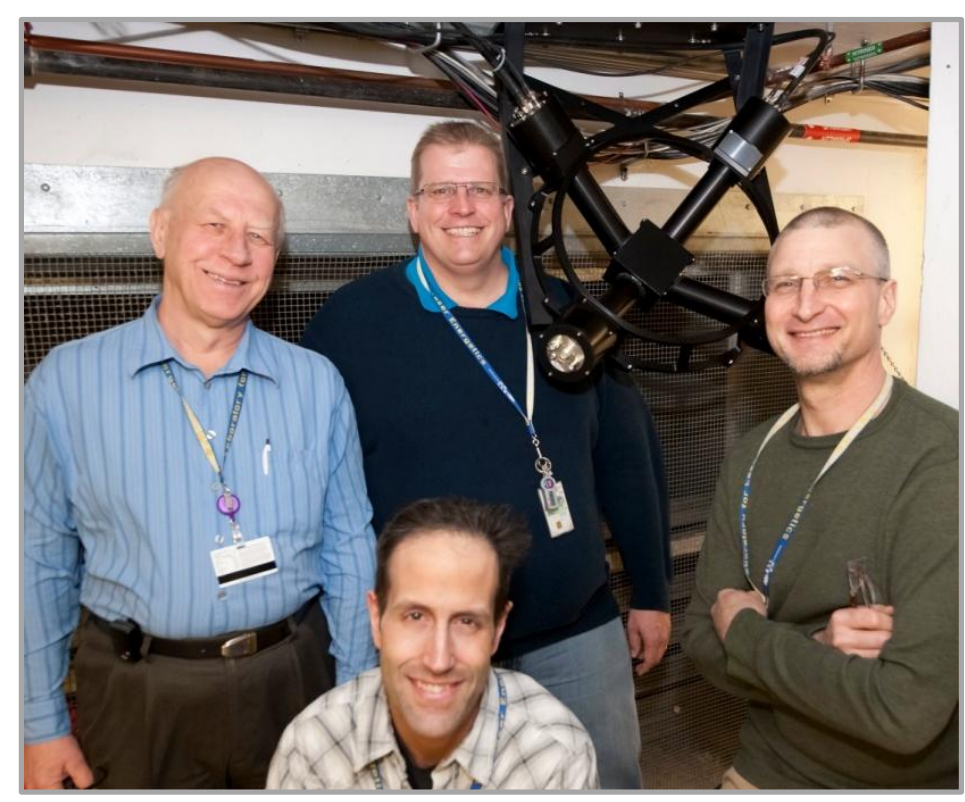

Figure 5-13. Neutron Time-of-Flight (top right) is shown with collaborators from $L L E$. 


\section{National Ignition Campaign Program Completion Report}

RAGS: The Radiochemical Analysis of Gaseous Samples diagnostic (see Figure 5-14) is used to collect and measure neutron activation products that are gaseous at room temperature. For example, noble gases such as krypton and xenon can be used as activation detectors by pre-loading low levels into the ablator. The resulting krypton and/or xenon isotopes produced can be collected and chemically fractionated very efficiently by cryogenic trapping. Isotopic analysis of the collected samples, when corrected for contributions from air, can be used to obtain quantitative data on multiple capsule performance parameters such as mix of the shell material into the fuel, asymmetry of implosion, shell and fuel areal density at peak emission, and neutron yield.

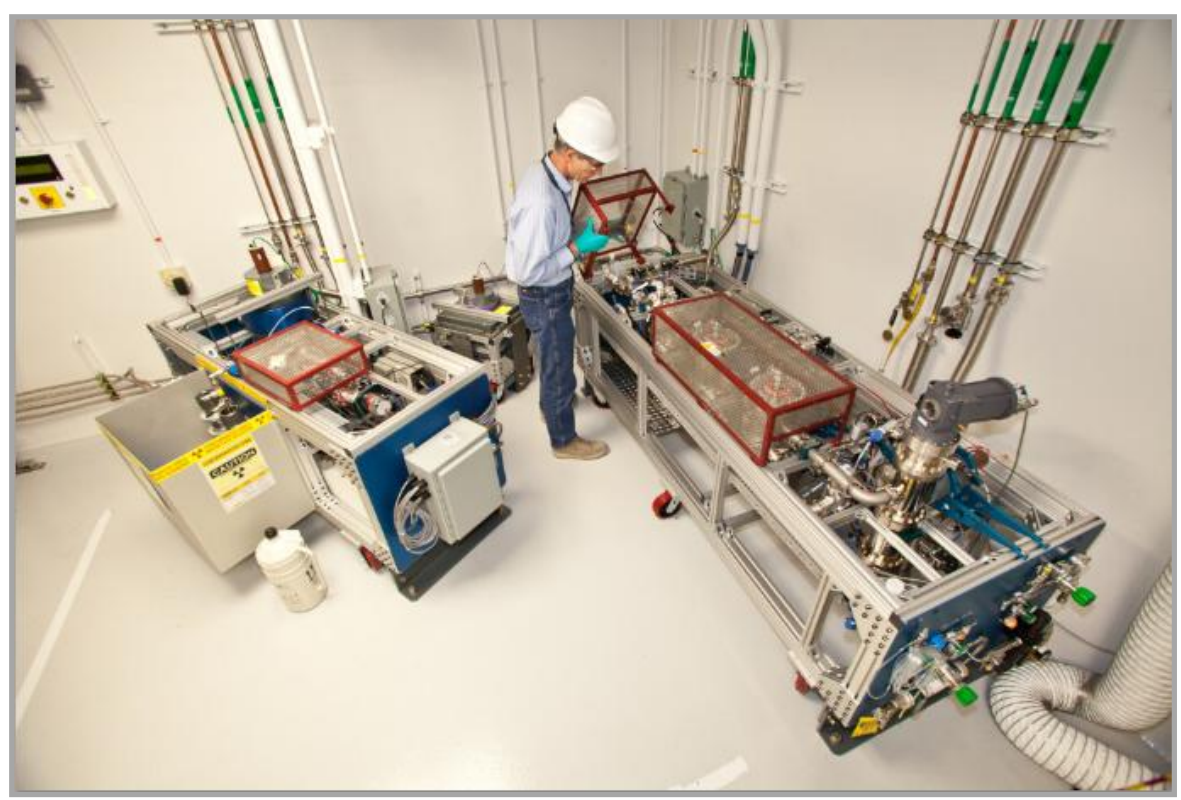

Figure 5-14. The Radiochemical Analysis of Gaseous Samples system collects and analyzes gases produced by NIF experiments.

SRC: The Solid Radiochemistry Collectors collect samples of solid target debris that may contain radioactive species produced by neutron activation. For example, gold from the hohlraums can be activated by neutrons and collected as a solid sample. SRC units placed about $50 \mathrm{~cm}$ from TCC as shown in Figure 5-5. The SRC units are removed post-shot and the presence of radioactive isotopes is determined by nuclear counting techniques in B151. The ratio of gold isotopes observed in SRCs has been used to determine areal density and some measure of mix.

WRF: Wedged Range Filters are used for D- ${ }^{3} \mathrm{He}$ gas-filled implosions (see Figure 5-15). The escaping thermonuclear protons lose energy in the compressed plastic. The energy spectrum of the escaping protons is measured by passing them through a wedge of material and measuring the energy of the protons after passing through various parts of the wedge with CR-39 track detectors. These WRF units are mounted at about 50 $\mathrm{cm}$ from TCC. The technique yields valuable data prior to the full compression of ablator. When the density of the ablator is about $200 \mathrm{mg} / \mathrm{cm}^{2}$ or higher, the protons are stopped in the ablator.

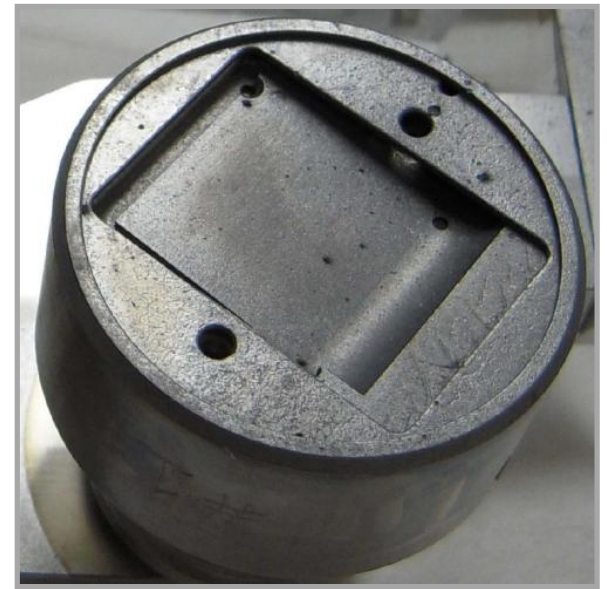

Figure 5-15. The Wedged Range Filter detector unit. 


\section{National Ignition Campaign Program Completion Report}

\section{c. Diagnostics of Mix}

\section{Table 5-5. Diagnostics of Mix}

\begin{tabular}{|l|l|l|l|l|}
\hline Acronym & Diagnostic & Contributors & Observable & Ref. \\
\hline Ross Filter Pair & Ross Filter Pair & LLNL & Mix & 34 \\
\hline $\begin{array}{l}\text { Supersnout II (multi- } \\
\text { wavelength) }\end{array}$ & $\begin{array}{l}\text { Multi-wavelength } \\
\text { X-ray Spectrometer }\end{array}$ & LLE/LLNL & Mix & 35 \\
\hline
\end{tabular}

Ross Filter Pairs: An array of "Ross filtered" pinholes records time integrated x-ray images after several different filter packs in the energy range from $8 \mathrm{keV}$ to $>20 \mathrm{keV}$. These give measures of the temperatureand density-sensitive bremsstrahlung emission from the imploding core. This data provides estimates of hot spot mass, mix mass, and pressure, as well as broadband, time-integrated absolute x-ray self-emission images of the imploded core.

Supersnout: Two four-channel curved crystal spectrometers are used to record with medium resolution K shell $\mathrm{x}$-rays from dopants such as germanium and copper in the plastic ablator once they mix into the hot spot and emit x-rays. The spectrometer is a snout that is attached to the front of a gated detector, and the data is recorded onto an image plate (see Figure 5-16).

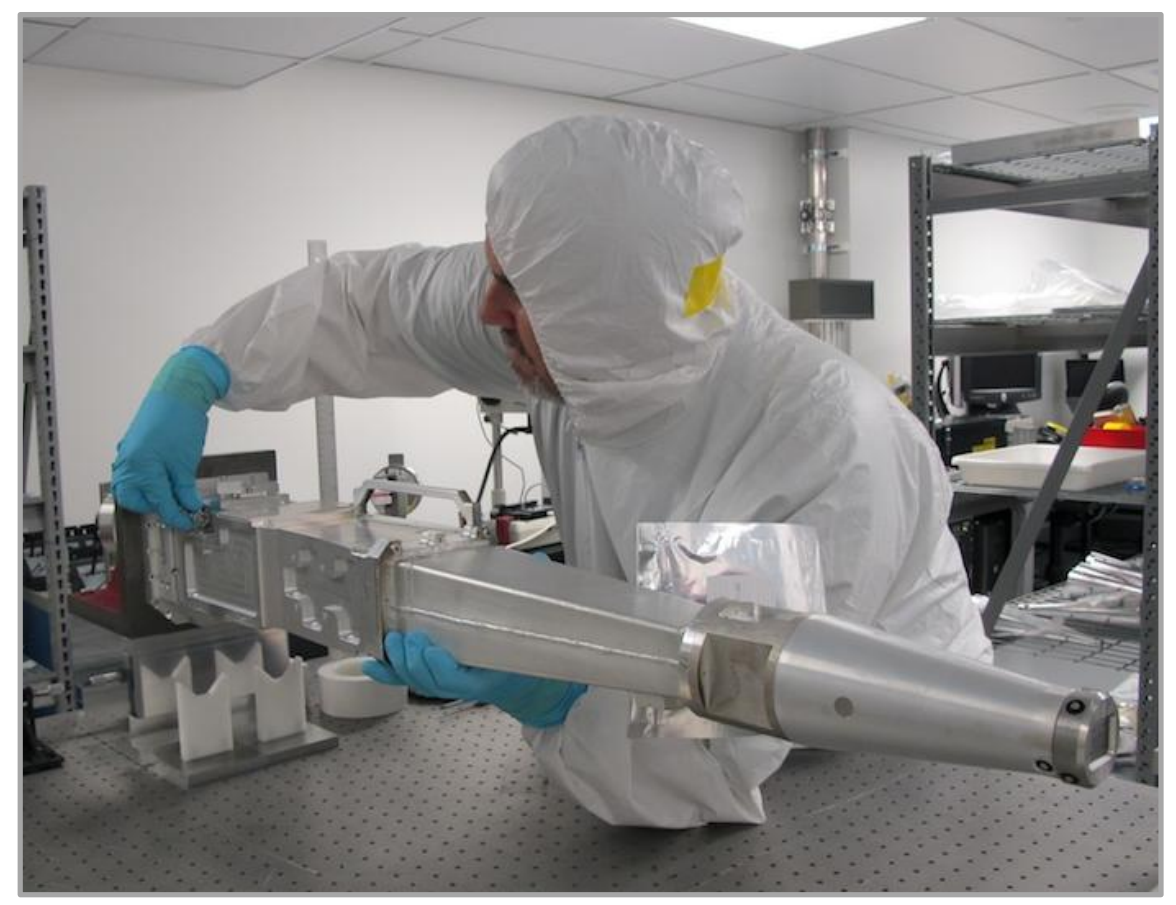

Figure 5-16. The Supersnout four-channel crystal spectrometer is attached to the recording box.

The locations of the target postioners (TARPOS and CryoTARPOS), DIMs, and some of the diagnostic systems on the NIF target chamber are shown in Figure 5-17. Also shown in the figure are FODI (Final Optics Damage Inspection system) and OPAS (Opposed Port Alignment System). 


\section{National Ignition Campaign Program Completion Report}

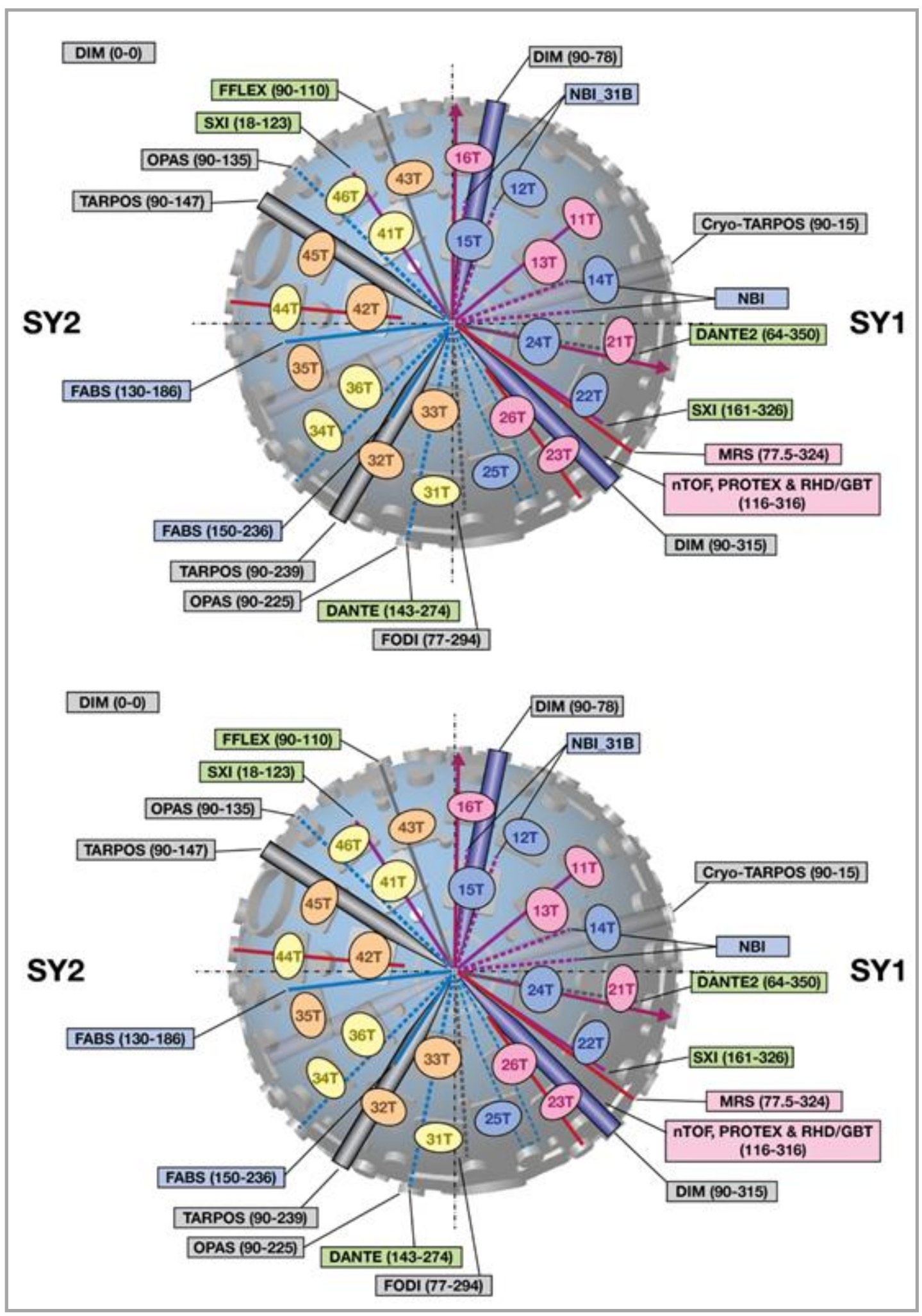

Figure 5-17. Top and bottom views of the NIF target chamber, showing the location of the quads and major diagnostic (Switchyards, SY1 and SY2, are shown on the right and left sides). NIF diagnostics are either constructed and deployed to a fixed location in the target chamber/bay or are fielded on a DIM. 


\section{National Ignition Campaign Program Completion Report}

\section{Designing New Diagnostics}

Before a new piece of equipment can be deployed at NIF, it must undergo a formal process, including a series of reviews. A member of the NIF Diagnostic Engineering team is assigned to assist each researcher in the design of hardware that can be readily fielded on the NIF. The NIF Diagnostic Engineering interface partner helps determine the appropriate review scope and deliverables using a risk-based graded approach. A NIF document is available that describes the diagnostic design process and design requirements [36].

\section{Target Diagnostics Control System}

Controls for target diagnostics are managed as part of the ICCS high-level architecture [37]. ICCS incorporates over a thousand front-end processors, servers, and workstations to control, diagnose, and fire the laser, as well as to integrate the suite of target diagnostics. Target diagnostics have unique control system requirements; they must be able to operate outside of the supervisory environment and be operated or calibrated in facilities other than NIF.

During NIC, the target diagnostics embedded control architecture was modified to use a single low-cost PC104 processor per device (e.g., a digitizer). The full diagnostic control is then composed within the supervisory software by aggregating the network-attached controllers. This approach simplifies the embedded software, improves reliability, and provides easy reuse of devices in other diagnostics.

\section{Diagnostic Control System}

The Diagnostic Control System (DCS) framework for NIF hardware and software was developed to:

- Reduce hardware and software costs;

- Increase efficiency by reusing software;

- Improve verification and test case coverage; and

- Speed up development turnaround for new diagnostics.

Each complex diagnostic typically uses an ensemble of electronic instruments attached to sensors, digitizers, cameras, and other devices. A diagnostic's supporting instruments (i.e., power supplies, cameras, and/or digitizers) are each maintained by a dedicated computer controller with generic DCS software customized to that instrument. Figure 5-18 illustrates this architecture for the Dante soft x-ray spectrometer diagnostic. The Dante diagnostic uses one DCS controller with software and interface hardware specific to the power supply and 20 more controllers with software and interface specific to the oscilloscope. The various DCS computers are located in standard 19 inch electronic racks in one of four diagnostic mezzanines adjacent to the NIF target area shielding wall, along with the

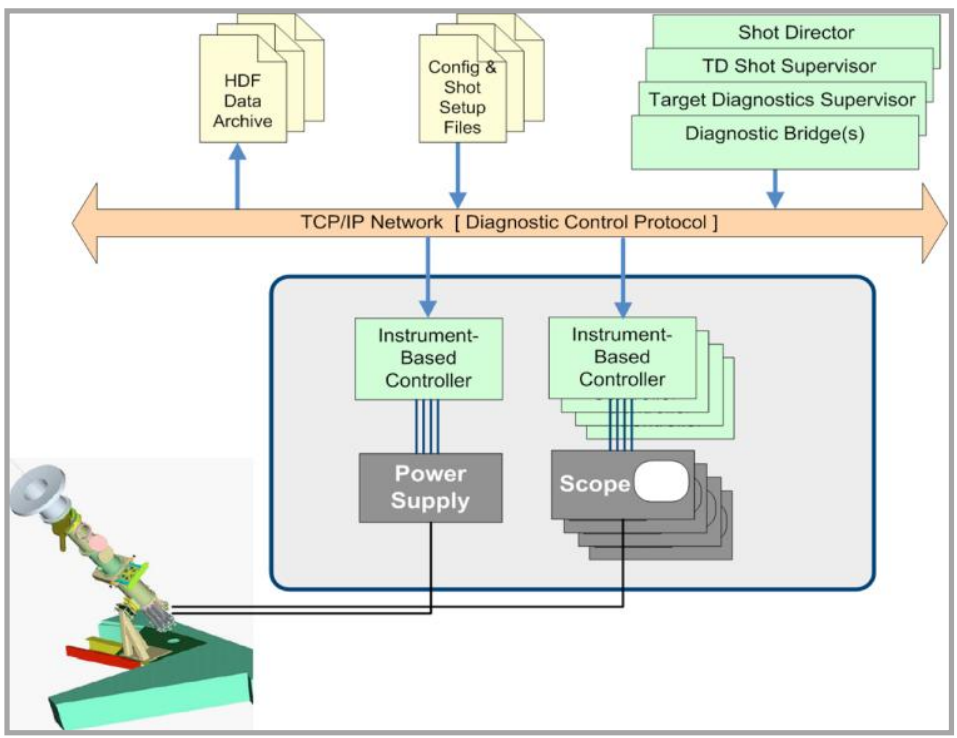

Figure 5-18. Diagnostic control system architecture as applied to Dante x-ray diagnostic. power supplies and digitizers. Computers are connected to the ICCS network through network switches in the diagnostic mezzanine. These controllers are diskless and boot from a file server over the network. Experimental data collected from cameras and digitizers by each controller are sent to the file server for processing and archiving. 


\section{National Ignition Campaign Program Completion Report}

Figure 5-19 illustrates the DCS framework, which instantiates objects that perform the following common functions. In the DCS architecture, each instrument is interfaced to an inexpensive Windows processor and JAVA application. The JAVA framework provides data management, control services, and operator GUI generation. DCS instruments are reusable by replication with reconfiguration for specific diagnostics in extensible markup language. Advantages include minimal application code, easy testing, and high reliability. Collaborators save costs by assembling diagnostics with existing DCS instruments.

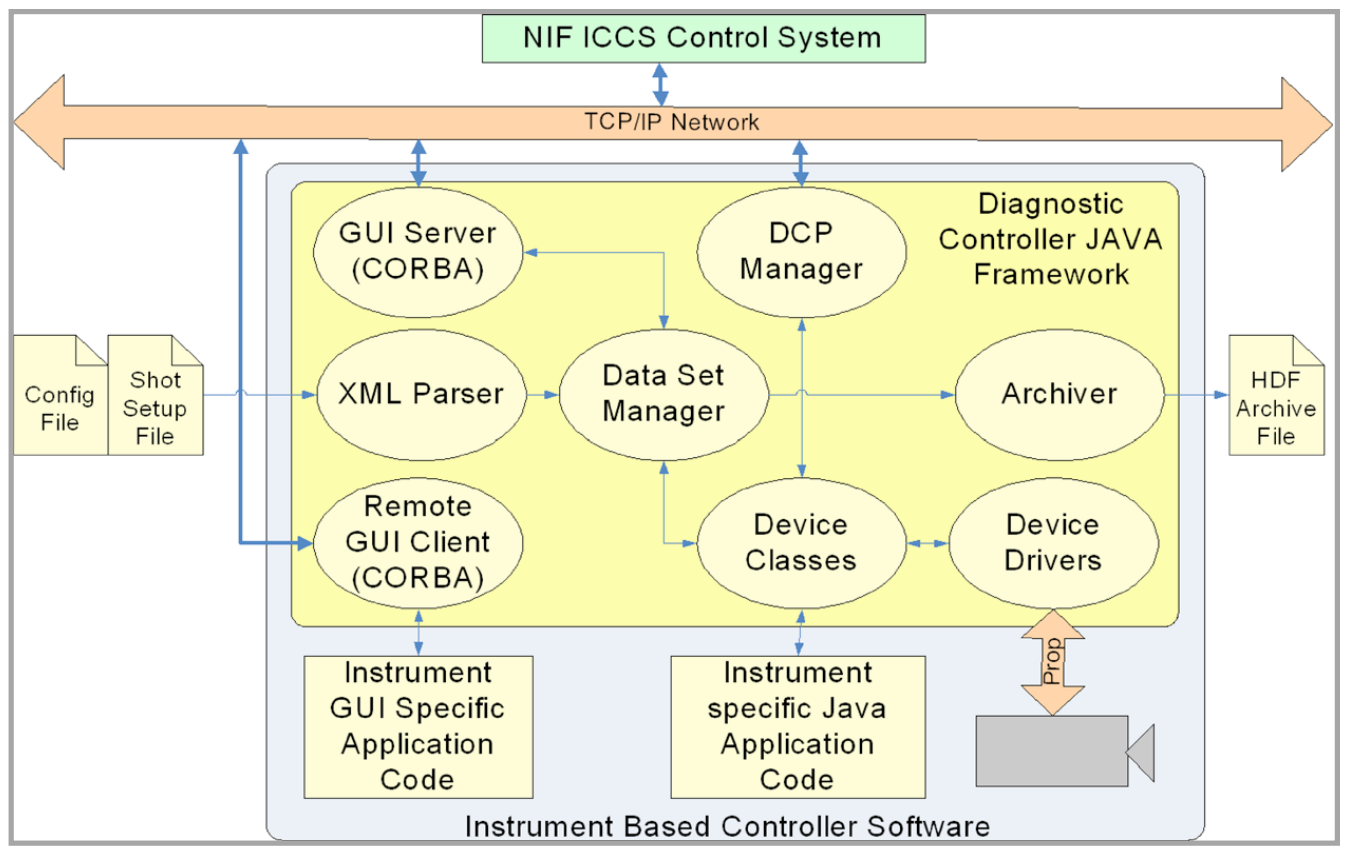

Figure 5-19. Diagnostic control system framework.

\section{Target Diagnostic Supervisor}

The ICCS target diagnostic subsystem is a target diagnostic front-end processor containing diagnostic bridges and supervisory and shot control software. Diagnostic bridges translate DCP protocol messages from each DCS instrument into ICCS CORBA-distributed objects. The target diagnostic supervisor uses these bridges to provide status and control of each DCS instrument, and groups the set of instruments for the diagnostics they support. The target diagnostic supervisor also provides the primary operator interface at the target diagnostic console in the control room. The shot supervisor executes macro-steps that are defined in a shot model for participating diagnostics on any given shot. Instrument configuration for a specific diagnostic and shot combination is established in configuration files by the responsible diagnostic engineer. After review of the post-shot data from a given diagnostic by the responsible diagnostic engineer, it is made available to the user via the Archive Viewer. See I.2, Systems Engineering, for further user interface and data access information.

\section{References}

1. J.L. Kline et al., "The first measurements of soft x-ray flux from ignition-scale hohlraums at the National Ignition Facility using DANTE," Rev. Sci. Instrum. 81, 10E321 (2010).

2. J.D. Moody et al., "Backscatter measurements for NIF ignition targets," Rev. Sci. Instrum. 81, 10D921 (2010).

3. E.L. Dewald et al., "Hot electron measurements in ignition relevant hohlraums on the National Ignition Facility," Rev. Sci. Instrum. 81, 10D938 (2010).

4. M.B. Schneider et al., "Images of the laser entrance hole from the static x-ray imager at NIF," Rev. Sci. Instrum. 81, 10E538 (2010). 


\section{National Ignition Campaign Program Completion Report}

5. M.B. Schneider et al., "Soft x-ray images of the laser entrance hole of ignition hohlraums," Rev. Sci. Instrum. 83, 10E525 (2012).

6. J.R. Kimbrough et al., "Standard design for National Ignition Facility x-ray streak and framing cameras," Rev. Sci. Instrum. 81, 10E530 (2010).

7. D.G. Hicks et al., "Streaked radiography measurements of convergent ablator performance," Rev. Sci. Instrum. 81, 10E304 (2010).

8. Y.P. Opachich et al., "X-ray streak camera cathode development and timing accuracy of the $4 \omega$ ultraviolet fiducial system at the National Ignition Facility," Rev. Sci. Instrum. 83, 10E123 (2012).

9. G.A. Kyrala et al., "Measuring symmetry of implosions in cryogenic hohlraums at the NIF using gated x-ray detectors," Rev. Sci. Instrum. 81, 10E316 (2010).

10. P.M. Bell et al., "Radiation hardening of gated x-ray imagers for the National Ignition Facility," Rev. Sci. Instrum. 81, 10E540 (2010).

11. A. Pak et al., "Diagnosing radiative shocks from deuterium and tritium implosions on NIF," Rev. Sci. Instrum. 83, 10E507 (2012).

12. N. Izumi et. al., "Experimental study of neutron induced background noise on gated x-ray framing cameras," Rev. Sci. Instrum. 81, 10E515 (2010).

13. V. Yu et al., "The National Ignition Facility neutron time-of-flight system and its initial performance," Rev. Sci. Instrum. 81, 10D325 (2010).

14. H. Rinderknect et al., "A novel particle time of flight diagnostic for measurements of shock- and compression-bang times in $\mathrm{D}^{3} \mathrm{He}$ and DT implosions at the NIF," Rev. Sci. Instrum. 83, 10D902 (2012).

15. D.H. Edgell et al., "South pole bang-time diagnostic on the National Ignition Facility," Rev. Sci. Instrum. 83, 10E119 (2012).

16. S.F. Khan et al., "Measuring x-ray burn history with the Streaked Polar Instrumentation for Diagnosing Energetic Radiation (SPIDER) at the National Ignition Facility," Proc. SPIE $\mathbf{8 5 0 5}$ (2012).

17. R.M. Malone et al, "Overview of the line-imaging VISAR diagnostic at the National Ignition Facility," Proc. SPIE 6342 (2007).

18. V. Smalyuk et al., "X-ray imaging in an environment with high-neutron background on National Ignition Facility Proc. SPIE 8144, 81440N (2011).

19. S.R. Nagel et al., "Dilation x-ray imager a new/faster gated x-ray imager for the NIF," Rev. Sci. Instrum. 83, 10E116 (2012).

20. H.W. Herrmann et al., "Diagnosing inertial confinement fusion gamma ray physics," Rev. Sci. Instrum. 81, 10D333 (2010).

21. D.B. Sayre et al., "Multi-shot analysis of the gamma reaction history diagnostic," Rev. Sci. Instrum. 83, 10D905 (2012).

22. R.J. Leeper et al., "Target diagnostic system for the national ignition facility," Rev. Sci. Instrum. 68, 868 (1997).

23. D.L. Bleuel et al., "Neutron activation diagnostics at the National Ignition Facility," Rev. Sci. Instrum. 83, 10D313 (2012).

24. E.N. Loomis et al., "Progress toward the development and testing of source reconstruction methods for NIF neutron imaging," Rev. Sci. Instrum. 81, 10D311 (2010).

25. R.A. Lerche et al., "National Ignition Facility neutron time-of-flight measurements," Rev. Sci. Instrum. 81, 10D319 (2010).

26. R. Tommasini et. al., "Development of Compton radiography of inertial confinement fusion implosions," Physics of Plasmas 18, 056309 (2011).

27. M. Gatu Johnson et al., "Neutron spectrometry-An essential tool for diagnosing implosions at the National Ignition Facility," Rev. Sci. Instrum. 83, 10D308 (2012). 


\section{National Ignition Campaign Program Completion Report}

28. J.A. Frenje et al., "Probing high areal-density cryogenic deuterium-tritium implosions using downscattered neutron spectra measured by the magnetic recoil spectrometer," Physics of Plasmas 17, 056311 (2010).

29. D.T. Casey et al., "The coincidence counting technique for orders of magnitude background reduction in data obtained with the magnetic recoil spectrometer at OMEGA and the NIF," Rev. Sci. Instrum. 82, 073502 (2011).

30. D.T. Casey et al., "Measuring the absolute deuterium-tritium neutron yield using the magnetic recoil spectrometer at OMEGA and the NIF," Rev. Sci. Instrum. 83, 10D912 (2012).

31. M.A. Stoyer et al., "Collection of solid and gaseous samples to diagnose inertial confinement fusion implosions," Rev. Sci. Instrum. 83, 023505 (2012).

32. G.P. Grim et al., "Prompt radiochemistry at the National Ignition Facility," Rev. Sci. Instrum. 79, 10E503 (2008).

33. A.B. Zylstra et al., "Charged-particle spectroscopy for diagnosing shock $\rho$ R and strength in NIF implosions," Rev. Sci. Instrum. 83, 10D901 (2012).

34. T. Ma et al., "Imaging of high-energy x-ray emission from cryogenic thermonuclear fuel implosions on the NIF," Rev. Sci. Instrum. 83, 10E115 (2012).

35. S.P. Regan et al., "Applied plasma spectroscopy: Laser-fusion experiments,” High Energy Density Physics 5, 234 (2009).

36. Diagnostic Design Guidance for the National Ignition Facility, NIF-5041482.

37. R.T. Shelton et al., "Target diagnostic control system implementation for the National Ignition Facility," Rev. Sci. Instrum. 81, 10E101 (2010). 


\section{National Ignition Campaign Program Completion Report}

\section{I.6-USER OPTICS}

The NIC WBS Element I.6, User Optics, includes the disposable debris shields for experimental campaigns; the capability, through user-requested optics, to modify the properties of the laser beam using CPPs, polarization-smoothing crystals, bandwidth, and spatial and temporal configurations; the optics inventory acquired for NIF operations; the process development for manufacturing and refurbishing user and final optics; and the ongoing optics continuous improvement program.

An essential effort within User Optics was the development of a strategy to recycle and reuse optics called "the Loop." The Loop involves inspecting all of the final optics after each shot, determining which optics have damage sites that require treatment (sites that are growing), removing these optics from the beamline, and subjecting them to appropriate mitigation technologies to repair the damage. With this strategy, when damage initiation occurs, the impact of damage growth is controlled and optics are recycled or refinished many times before being discarded. Implementation of the Loop required developing processes and facilitizing and commissioning complex equipment and cleanrooms to refurbish and mitigate damage sites. Significant cost savings for NIF operations are achieved by using a mixture of refurbished, vendor refinished, and eventually, new replacement optics.

\section{A. User-Specific and Operational Optics}

Critical optics have been developed and manufactured within the NIC to support experiments for the ignition program on NIF. User-specific ignition optics consist of Disposable Debris Shields (DDSs), CPPs, Polarization Rotators (PRs), and NIF operational spares supporting high-energy, high-power operation.

\section{Disposable Debris Shields}

The $3 \omega$ fused silica optics within the FOA have extremely challenging optical specifications, making them difficult to fabricate and thus relatively expensive. To protect these FOA optics from shrapnel and vaporized target materials generated during a laser shot, DDS optics are positioned at the output of the FOAs. DDSs are routinely monitored; as soon as the light transmission becomes sufficiently degraded due to surface debris, they are automatically removed and replaced, without disrupting normal shot operations. DDSs are thin optics ( $\sim 3 \mathrm{~mm}$ thick) that can be manufactured for a fraction of the cost of the optics they protect. For example, one DDS can be fabricated at less than a hundredth the cost of a WFL. During NIC, a DDS factory was designed, constructed, commissioned, and brought into production to supply up to 8,000 DDS optics per year to support NIF operations (see Figure 6-1).

\section{Continuous Phase Plates}

The CPPs are user-defined optics that modify the focal spot size of each NIF beam. For the ignition program, the CPPs produce the

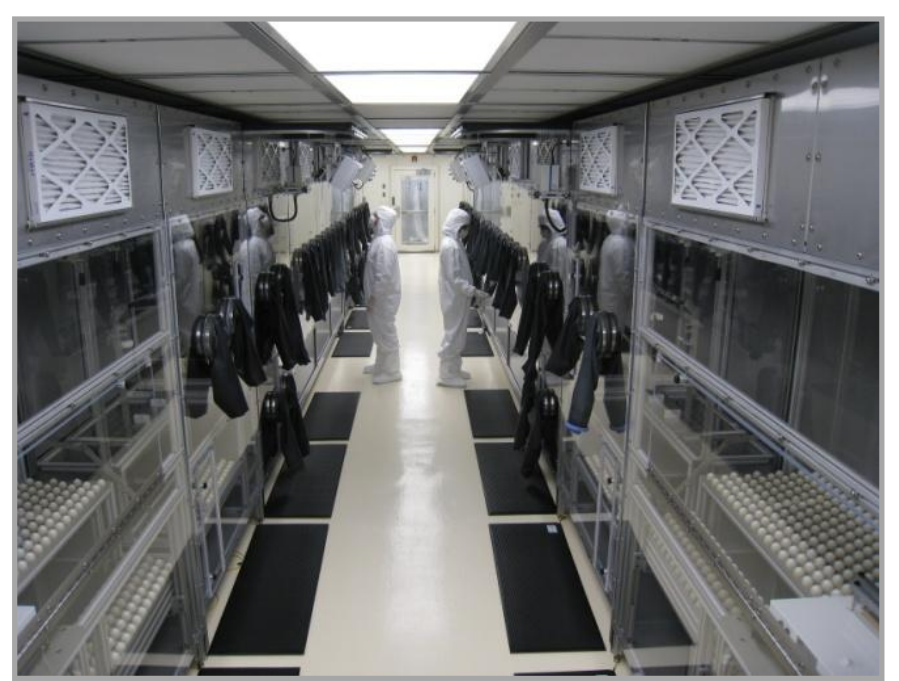

Figure 6-1. Dual-line Disposable Debris Shield cleaning and coating facility at Schott Corp. required spot size within the hohlraum. CPP optics use a free-form diffractive topology prescription that is imprinted into the surface using magneto-rheological finishing (MRF) technology to provide the desired beam spot size and uniformity [1,2]. During NIC, four large-aperture MRF machines were purchased and installed at the competitive vendors Zygo and ITT Excelis to fabricate CPPs for the NIF user community. A total of three sets of 96 CPPs plus spares were manufactured as part of the NIC. In 


\section{National Ignition Campaign Program Completion Report}

addition to fabricating CPP optics, MRF technology has also been integrated into the $3 \omega$ thin flat finishing process to produce optics highly resistant to damage by $3 \omega$ laser light and with low subsurface fracture [3].

\section{Polarization Rotator Crystals}

Two beams in each FOA have PR optics that rotate the polarization 90 degrees to accomplish polarization smoothing on the target. During the NIC, PR optics were designed and a phase retardation instrument was constructed at Cleveland Crystals Incorporated (CCI) to validate performance to specification. The PRs are optics made from potassium dihydrogen (KDP) crystals and are similar to switch crystals used in NIF's Pockels cells. Both PRs and switch crystals are harvested from large KDP crystal boules cut perpendicular to the $\mathrm{z}$-axis ${ }^{\mathrm{dd}}$ (z-cut crystals). KDP boules that are too small to harvest second-harmonicgenerator (SHG) crystal optics, but large enough to fabricate switch crystals can be used to fabricate PR crystals. Additionally, some of the deuterated-KDP (DKDP) boules that were too small for thirdharmonic-generator (THG) crystals also can be used to fabricate PR crystals. An advantage of the DKDP material is the $1 \omega$ absorption is about $5 \%$ lower than KDP, thus reducing the necessary $1 \omega$ drive energy in the most stressed beams on NIF. Ninety-six PR crystals plus operational spares were fabricated, cleaned, coated, assembled, and installed on NIF.

\section{Operational Spares}

During NIC, operational spares of all of the large optics were fabricated to augment the $3 \%$ spares made during NIF construction. The percentage of operational spares in the NIC plan was determined on the basis of several factors:

- Location of the optic $(1 \omega, 2 \omega$, or $3 \omega)$;

- Operational fluence seen by the optic;

- Inherent laser damage resistance of the optic; and

- Fabrication time needed to replace optics.

The situation was different for laser glass [4-10] because the glass melters at Schott and Hoya were completely dismantled in 2011 and the valuable platinum melter liner was recovered and returned to the Department of the Treasury and the Department of Energy (DOE). Since the costs associated with restarting laser glass melting is prohibitive (tens of millions of dollars), approximately 10,000 total slabs were melted under NIC for the combination of NIF and the Laser MegaJoule (LMJ) facilities. ${ }^{\text {ee }}$ Currently, NIF has 3,072 slabs installed on NIF, populating an 11:5 configuration; NIF could be expanded to an 11:7 configuration in the future, using 3,456 slabs in total, to increase to the available $3 \omega$ energy on target.

\section{Competitive Optics Vendors}

Multiple sources were developed and facilitized as part of the NIF project and then the NIC program for fused silica $3 \omega$ optics (thin flats including Grating Debris Shields [GDSs], CPPs, and WFLs) (see Figure 6-2). These efforts enabled competitive procurement contracts with multiple vendors that minimized optics fabrication costs and reduced the schedule and delivery risk to NIF operations. Likewise, multiple fused silica glass blank vendors were qualified to create inclusion-free optics with a high $3 \omega$ laser threshold, resulting in a competitive vendor base of three vendors: Asahi Glass Company, Hereaus, and Nikon. ITT Excelis and Zygo are both qualified to fabricate fused silica $3 \omega$ optics flats.

\footnotetext{
dd The natural habit (shape) of a KDP crystal boule has a square base defined by the $\mathrm{x}$ and $\mathrm{y}$ coordinates with a pyramid cap.

ee The production of laser glass slabs for NIF and LMJ was jointly funded by the U.S. DOE and CEA.
} 


\section{National Ignition Campaign Program Completion Report}

Facilitization of ITT Excelis as an alternate competitive WFL vendor to Tinsley also occurred during the NIC, with the first pilot production of WFLs to be completed by the end of FY2012.

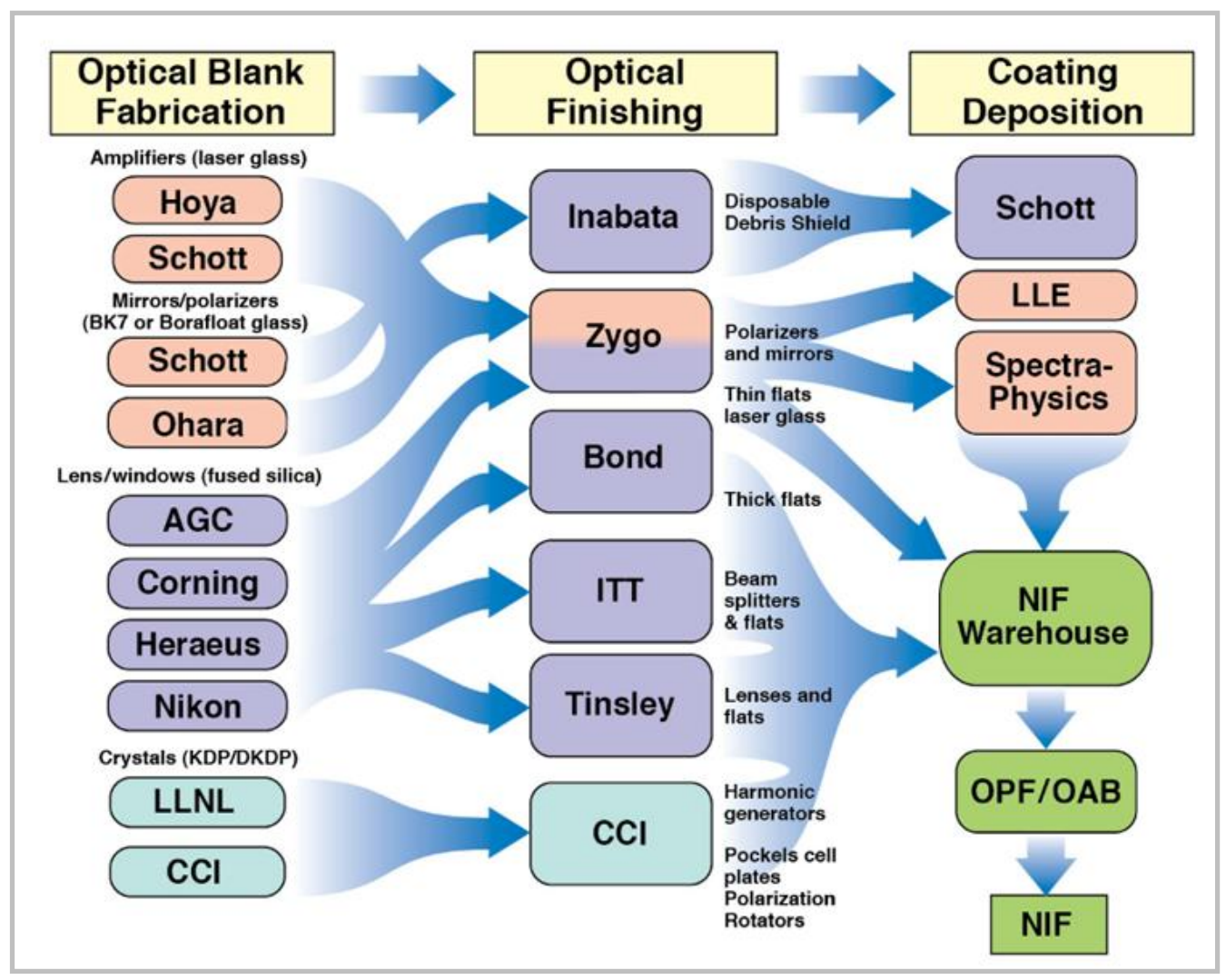

Figure 6-2. LLNL has established a partnership with the large-aperture optics industry over the past 30 years. Competitive vendors were used to fabricate and process most of NIF's optics.

\section{B. Loop Optics Recycling Strategy}

Development of the loop optics recycling strategy has allowed the high-cost optics in the Final Optic Assembly (i.e., WFL and GDS fused silica optics, SHG and THG, and KDP and DKDP crystal optics) that are subjected to $3 \omega$ light to operate consistently and predictably at a level significantly above their damage growth threshold (see Figure 6-3). The recycling is actually an assembly of loops, with decision points for moving from one loop to another. The innermost loop allows the laser to be fired repeatedly, with in-situ optics inspection between shots, without any other action required until the size of any observed damage site has reached the point where action has to be taken to prevent that site from reaching a size too large to apply mitigation technologies. At that point, if the operating situation of the laser allows, a shadow blocker can be electronically placed in the Injection Laser System to protect the site location, as identified by the in-situ optics inspection system. The laser can then continue to be fired, with optics inspections occurring between each high fluence shot. When the number of shadow blockers begins to significantly reduce the energy that can be delivered by the laser, one or more of the optics in the quad of interest are removed, sent to a mitigation facility for repair, and replaced by a near-perfect optic. If an optic has reached a damage state that cannot be mitigated at LLNL, it must be sent back to the vendor for refinishing, or discarded and replaced by a new optic. 


\section{National Ignition Campaign Program Completion Report}

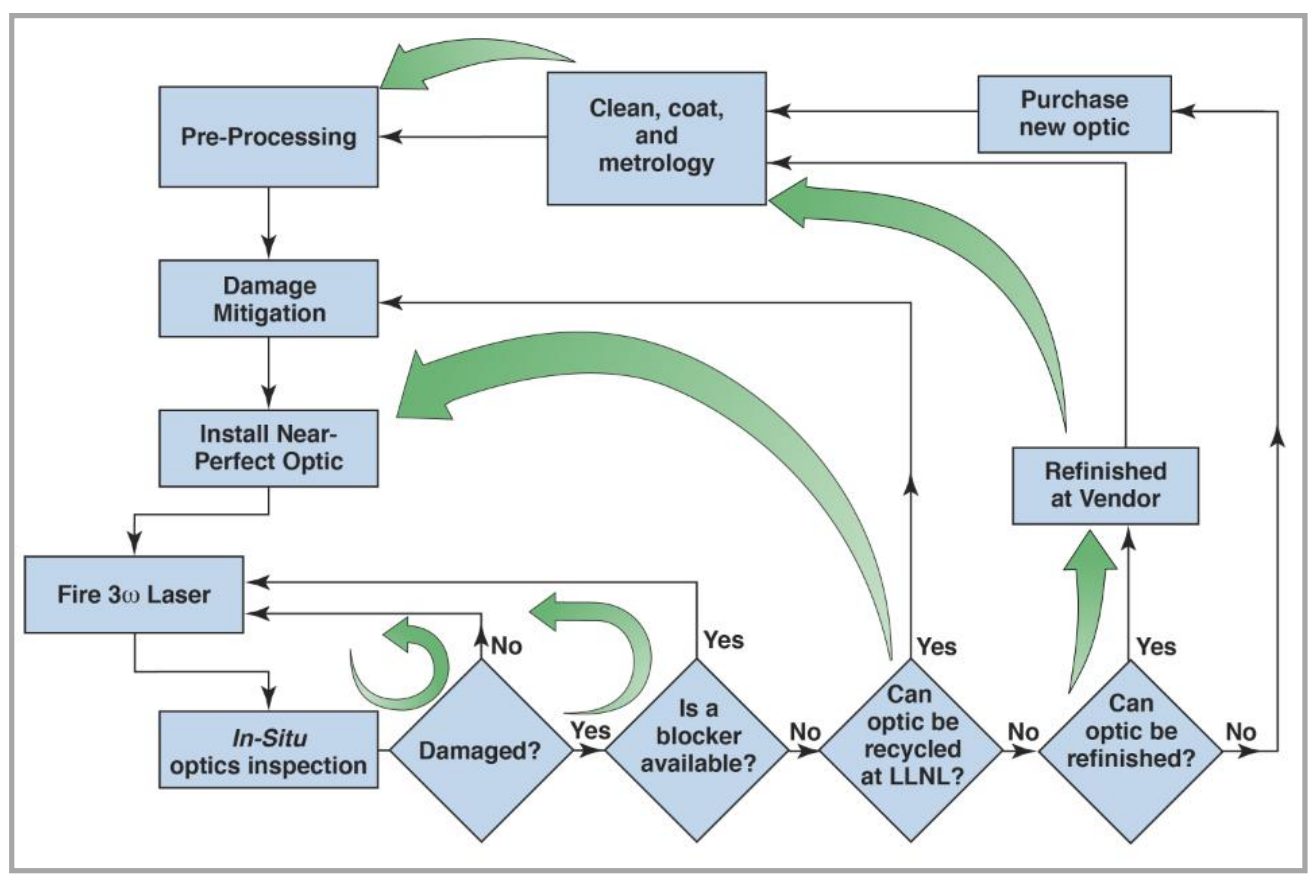

Figure 6-3. The Loop strategy allows NIF to operate cost effectively by using a mixture of refurbished, vendor refinished, and eventually, new replacement optics.

Capabilities that had to be developed in order for the optics recycling loop to work included:

- Optics that are resistant to damage initiation.

- An in-situ ability to identify the location of damage sites on each individual optic.

- The electronic ability to block incident light on a threatened damage site.

- The ability to remove, replace, and repair a damaged optic.

Once recycling loop capabilities were in place at a minimal level, the loop could be used to support operation of the laser and allow its performance to be incrementally improved as the capabilities of the loop improved. This same characteristic that allows utilization of evolutionary improvements in capability can also provide flexibility for operations planning. A choice can be made between lowering operating cost and operating the laser at higher performance levels.

Implementing the loop ultimately resulted in the development of:

- Higher quality and more robust bulk materials for the optical blanks.

- Advanced finishing techniques and processes.

- Post-processing technologies to improve optic $3 \omega$ laser resistance.

- Techniques to place a fiduciary marker on an optic.

- Equipment to identify and characterize flaws in bulk optical material and on optically polished surfaces [11].

- Final optics damage inspection system used to track in-situ damage growth on NIF.

- Programmable spot blockers to project shadows over damage sites to arrest damage growth [12].

- Mitigation protocols and facilities to repair damage sites [13-15].

- Optics exchange process and operational strategy that includes reprocessing of the used optics on NIF.

- Comprehensive program to understand the dynamics of laser damage that includes mapping the pulse shape and wavelength dependence of damage initiation and growth. 


\section{National Ignition Campaign Program Completion Report}

The time scale of the loops increases as one moves toward the outer loops. The inner two loops, inspection and decisions regarding the placement of the electronic shadow blockers, take hours. The cycle time for the mitigation loop is about 2 weeks. As of September 2012, recycling of fused silica optics (the WFLs and the GDSs) through the mitigation loop was being done at a rate of about 150 optics per month; thus, about a third of these optics are being sent through the mitigation loop each month. Some of the NIF fused silica optics have been through this mitigation recycling loop as many as 7 times. If an optic accumulates more damage than can be readily handled with blocking and mitigation, it is sent out for refinishing. Refinishing saves the cost of a new optic blank. About 15 optics/month are sent into this route. The cycle time for refinishing is about 6 months. Finally, completely new optics

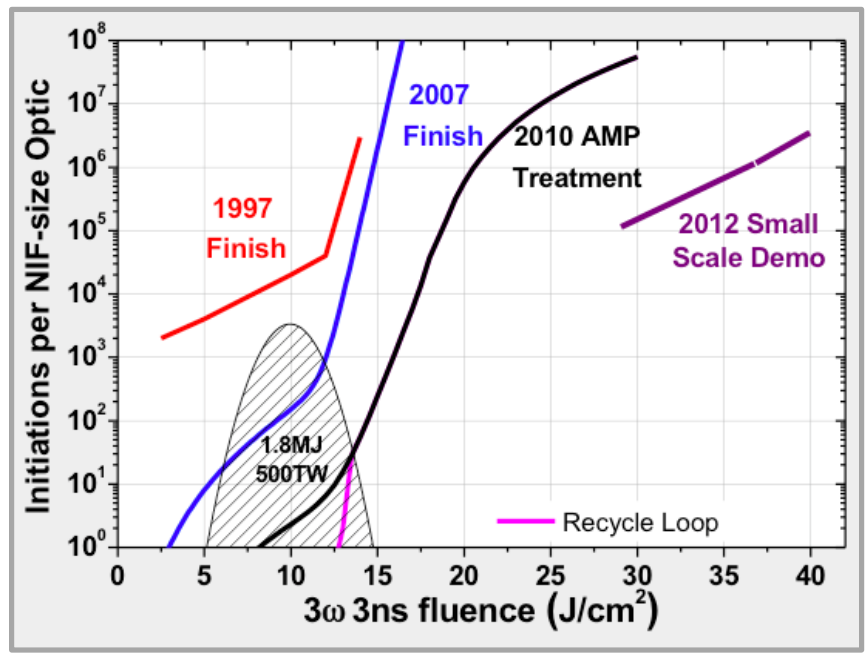

Figure 6-4. The optics development program has significantly improved $3 \omega$ optics damage rates over the past 15 years, as shown by the reduction in damage density as a function of $3 \omega$ fluence. are purchased to replace those that cannot be refinished, at a rate of about 10 per month. The cycle time for receiving a new optic from a currently contracted vendor is about one year.

Some of the loop achievements are highlighted in subsequent subsections.

\section{Advanced Optics Fabrication}

An effort to understand, on a fundamental level, the cause of laser-induced damage in materials (specifically KDP crystals and fused silica) in terms of both damage initiation and growth was undertaken as an institutionally supported research and development program (see Figure 6-4). For fused silica, this has produced an understanding of damage mechanisms (adsorbing impurities and fractures) [16] and provided a linkage between damage threshold and fast defect photoluminescence (PL) spectroscopy [17]. This linkage has further enabled the development of fast PL $(<<1 \mathrm{~ns})$ as a non-destructive precursor diagnostic for laser damage. The technique has become invaluable for assessing the effectiveness of mitigation technologies.

A science-based development program based on fracture mechanics and tribology was initiated concurrently at the NIF optics vendors to assess and improve the manufacturing processes used to make NIF-scale optics (see Figure 6.5). As a result, subsurface damage imparted during each step of the fabrication process can now be measured that allows process steps to be modified or even eliminated to improve quality. Also during NIC, further development of conventional DKDP crystal growth at our vendor CCI has resulted in fewer impurities incorporated within the bulk crystals. This, in turn, has significantly increased the $3 \omega$ laser damage resistance of the bulk materials. Overall, process 


\section{National Ignition Campaign Program Completion Report}

improvements guided by our science-based program have resulted in optics with demonstrably higher damage thresholds with a significantly reduced likelihood of damage initiation.

\section{Advanced Mitigation Process}

Mitigating technologies are processes that remove localized flaws (damage precursors) and leave behind mitigation morphologies that are benign with respect to downfield modulation or intensification of laser light. Further, the mitigation sites remain stable and do not change under continued laser exposure. Our science-based program has achieved considerable progress in developing robust mitigation processes. All of the $3 \omega$ fused silica optics are processed with a chemical-based Advanced Mitigation Process (AMP) that removes atomic defects from within tiny subsurface fractures caused by polishing and prevents the removed material (chemical species) from adhering to or on the surface $[18,19]$ (see Figure 6-6). Previous technologies could accomplish the former but left material on the surface that absorbed laser light and resulted in damage initiation. The AMP, originally developed under Laboratory Directed Research and Development (LDRD) funding, was scaled up for full-aperture NIF optics, and new equipment and protocols were incorporated into the NIF optics processing facility as part of NIC. A full complement of 192 WFL and GDS optics plus operational spares has been processed using AMP and installed on NIF. A different mitigation process was developed for the KDP frequency conversion crystal optics that are exposed to $3 \omega$ laser light (i.e., SHG and THG). This process involved laser conditioning the bulk KDP through slowly ramped, low-fluence laser exposure. KDP optics are exposed to a gentle fluence ramp that significantly alters possible damage precursors as part of their commissioning on NIF.

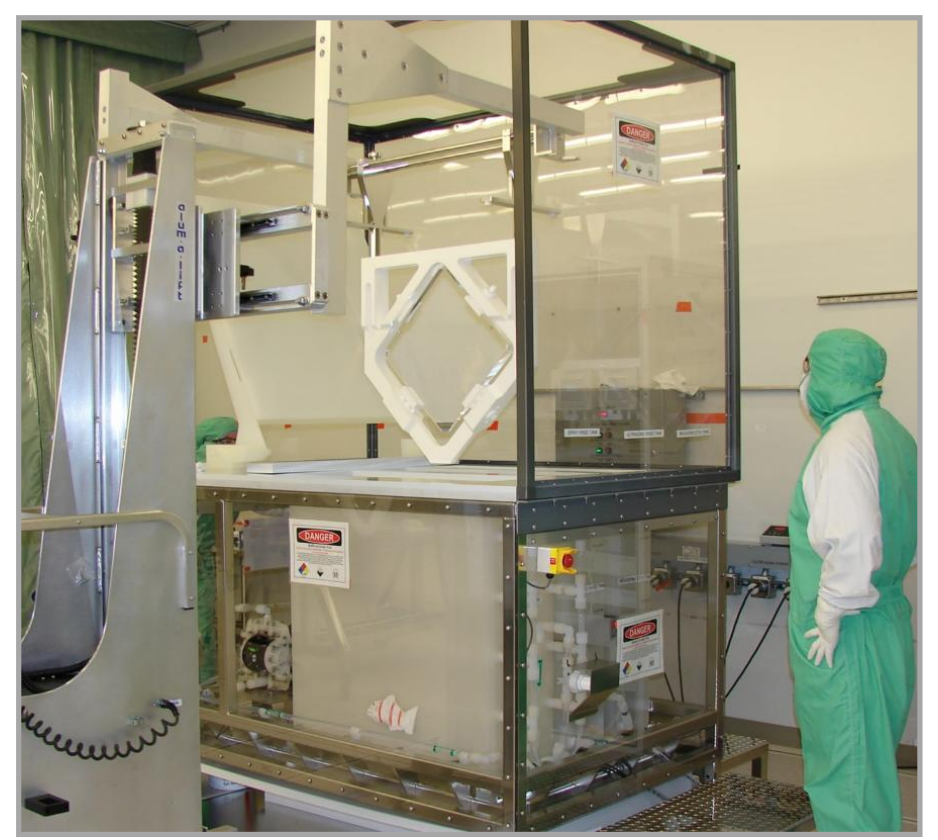

Figure 6-6. Advanced mitigation process station for increasing $3 \omega$ damage resistance of optic surface scratches.

\section{Optics Processing and Mitigation Facility}

Our science-based development program has guided the deployment of mitigation protocols that can repair damage sites on both fused silica [13,14] and KDP/DKDP [15] optics. An Optics Mitigation Facility (OMF) was built and machine tools designed, fabricated, and commissioned (see Figure 6-7). A total of four fused silica and two crystal mitigation stations were constructed to support NIF high-energy, high-power operation. OMF operation has been staffed for two full shifts to meet the required process rate.

The fused silica machines use a $\mathrm{CO}_{2}$ laser in a spiral pattern to ablate material around the site, leaving a small cone-shaped pit in the optics surface. The geometry of the cone and the laser machining parameters have been specifically optimized to minimize downfield light intensification. A high-speed single diamond crystal is used in the crystal mitigation stations to create the conical pits. The machines are well engineered so that the processing steps are extremely repeatable and robust. 


\section{National Ignition Campaign Program Completion Report}

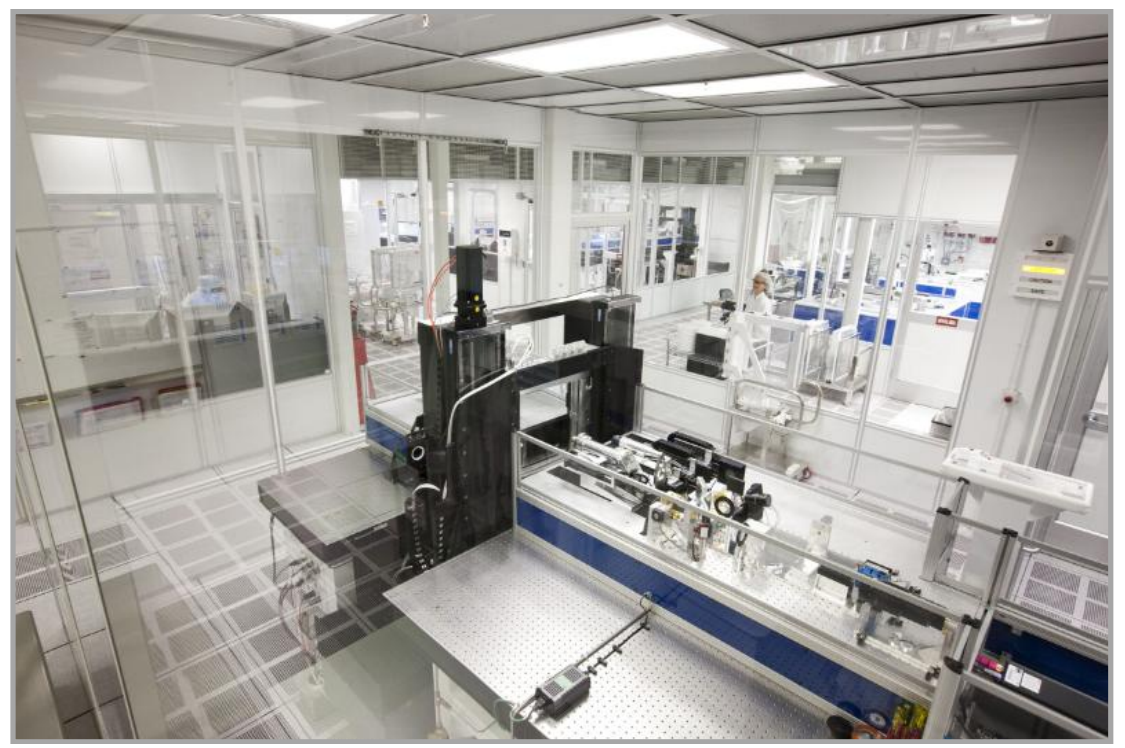

Figure 6-7. Optics Processing and Mitigation Facility.

\section{Flaw Identification and Characterization}

Every optics surface and potential damage initiation site within the final optics assembly, either from the manufacturing process or due to initiation of growing laser damage from NIF operations, is characterized. The Final Optics Damage Inspection System (FODI) camera works by rotating to sequentially stare down each individual beamline and focus on each individual optic to identify and size possible damage sites (see Figure 6-8). It can accurately locate the position of each damage site with the aid of fiducial markers that are placed on all optics outside of their active beam area. FODI is assisted in identifying the location of each damage site by edge illumination that can be turned on independently for each optic location. A second production optics inspection instrument was constructed and commissioned to increase the throughput of optics and to eliminate a single point of failure in optics processing.

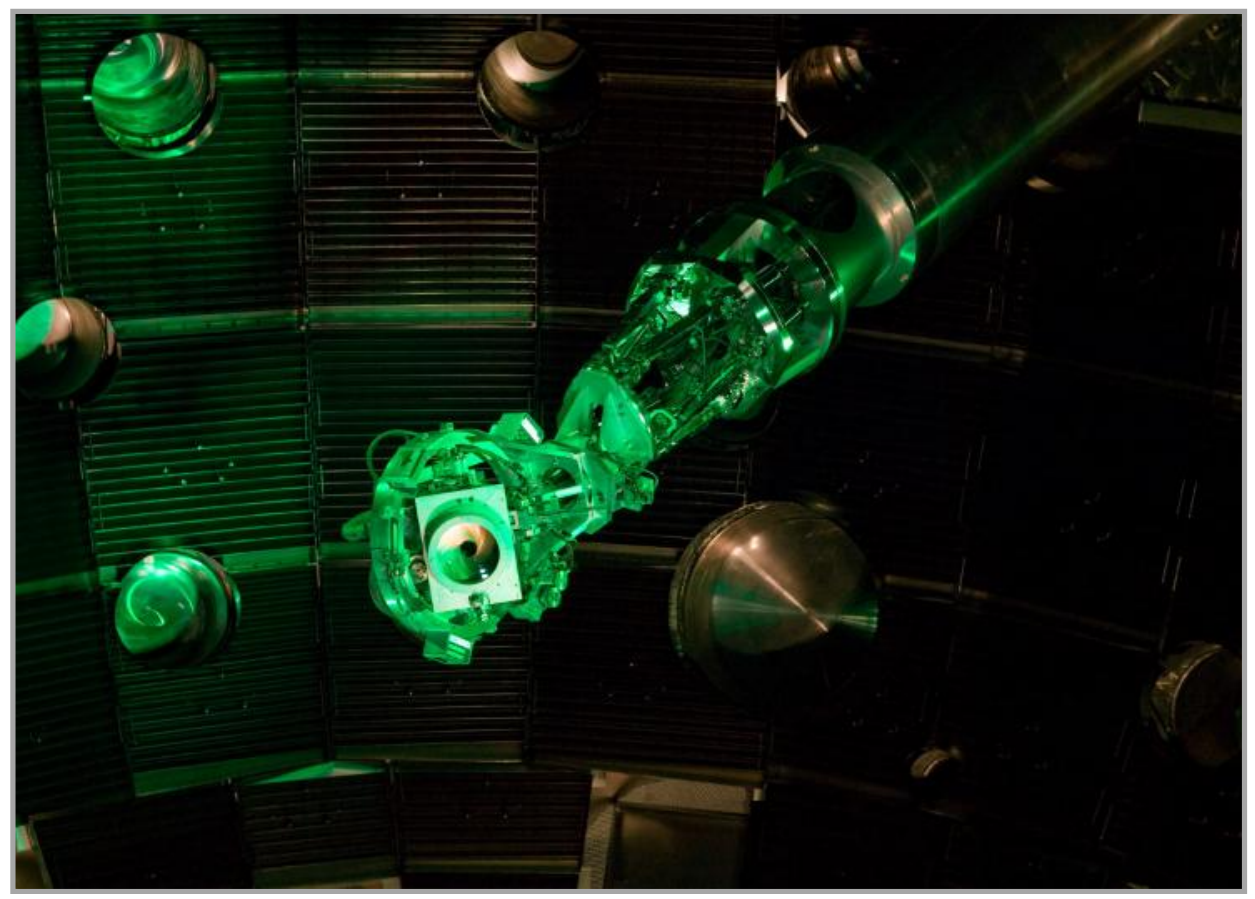

Figure 6-8. FODI, the in-situ damage inspection system for NIF $3 \omega$ optics. 


\section{National Ignition Campaign Program Completion Report}

\section{Purged Storage}

A detritiation process was developed through an internal LDRD-funded effort to reduce the tritium level on optics to below the free release limit. A purged storage facility was designed, constructed, and commissioned around this process. All optics that are removed from NIF that require either mitigation or refinishing as part of the recycling loop pass through the purged storage facility before they can be released and moved to other locations as part of normal NIF operations.

In summary, over the course of the NIC, facilities have been put into place and commissioned to construct user optics and enable the Loop recycling program. Today these facilities are actively processing optics to support routine high-energy (1.8 MJ), high-power (500 TW) experiments on NIF.

\section{References}

1. S.N. Dixit et al., "Designing fully continuous phase screens for tailoring focal plane irradiance profiles," Optics Letters 21 (21), 1715 (1996).

2. J.A. Menapace, "Developing Magnetorheological Finishing (MRF) Technology for the Manufacture of Large-Aperture Optics in Megajoule Class Laser Systems," Laser Induced Damage in Optical Materials: SPIE Proc. 7842, 1W-1-14 (2010).

3. J.A. Menapace et al., Combined advanced finishing and UV laser conditioning process for producing damage resistant optics, U.S. Patent 6,920,765, July 26, 2005.

4. J.H. Campbell et al., "High-Power Solid-State Lasers: a Laser Glass Perspective," International Journal of Applied Glass Science 2(1), 3 (2011).

5. J.H. Campbell and T.I. Suratwala, "Nd-doped phosphate glasses for high-energy/high-peakpower lasers," Journal of Non-Crystalline Solids 263, 318 (2000).

6. J.H. Campbell et al., "Continuous melting of phosphate laser glasses," Journal of Non-Crystalline Solids 263, 342 (2000).

7. P.R. Ehrmann et al., "Optical loss and $\mathrm{Nd}^{3+}$ non-radiative relaxation by $\mathrm{Cu}, \mathrm{Fe}$ and several rare earth impurities in phosphate laser glasses," Journal of Non-Crystalline Solids 263, 251 (2000).

8. J.S. Hayden et al., "Surface tensile layer generation during thermal annealing of phosphate glass," Journal of Non-Crystalline Solids 263, 228 (2000).

9. T.I. Suratwala et al., "Effects of OH content, water vapor pressure, and temperature on subcritical crack growth in phosphate glass," Journal of Non-Crystalline Solids 263, 213 (2000).

10. T.I. Suratwala et al., "Polishing slurry induced surface haze on phosphate laser glasses," Journal of Non-Crystalline Solids 351, 2091 (2005).

11. F.L. Ravizza et al., "Process for rapid detection of tratricidal defects on optics using line scan phase-differential imaging," Laser-Induced Damage in Optical Materials: Proc. SPIE 7504, 75041B (2009).

12. J. Heebner, "A programmable beam shaping system for tailoring the profile of high fluence laser beams," Laser-Induced Damage in Optical Materials: Proc. SPIE 7842, 78421C (2010).

13. I.L. Bass et al., "An improved method of mitigating laser-induced surface damage growth in fused silica using a rastered pulsed $\mathrm{CO}_{2}$ laser, in Laser-Induced Damage," Optical Materials: Proc. SPIE 7842, 784220 (2010).

14. J.J. Adams et al., "Results of applying a non-evaporative mitigation technique to laser-initiated surface damage on fused silica," Laser-Induced Damage in Optical Materials: Proc. SPIE 7842, 784223 (2010).

15. P. Geraghty, "Surface damage growth mitigation on KDP/DKDP optics using single-crystal diamond micro-machining ball end mill contouring," Laser-Induced Damage in Optical Materials: Proc. SPIE 6403, 64030Q (2006).

16. P.E. Miller et al., "Fracture-induced subbandgap absorption as a precursor to optical damage on fused silica surfaces," Opt. Lett. 35, 2702 (2010).

17. T.A. Laurence et al., "Metallic-like photoluminescence and absorption in fused silica surface flaws," Appl. Phys. Lett. 94, 151114 (2009). 


\section{National Ignition Campaign Program Completion Report}

18. T.I. Suratwala et al., "HF-Based Etching Processes for Improving Laser Damage Resistance of Fused Silica Optical Surfaces," J. Am. Ceram. Soc. 94, 416 (2011).

19. L. Wong et al., "The effect of $\mathrm{HF} / \mathrm{NH}_{4} \mathrm{~F}$ etching on the morphology of surface fractures on fused silica," Journal of Non-Crystalline Solids 355, 797 (2009). 


\section{I.7-PERSONNEL AND ENVIRONMENTAL PROTECTION SYSTEMS}

The NIC WBS Element I.7, PEPS, includes the systems required to contain and process hazardous and radioactive materials and protect workers and the public from radiation produced by the target experiment; systems implemented to decontaminate and, as needed, to dispose of contaminated components and experimental byproducts; and shielding systems implemented to reduce radiation doses to workers and to minimize radioactivity generated from neutron activation of materials. Additionally, the necessary processes, procedures, and training elements that together allow safe operations on NIF while producing nuclear yield and using tritium, depleted uranium, and beryllium comprise PEPS.

NIC uses tritium, a radioactive material, as part of the DT target fuel used in ignition experiments. During these experiments, tritium reacts with deuterium to produce helium and a very energetic neutron. These energetic neutrons further interact with materials in the target and in and around the target chamber. Some of the interactions lead to activation of these materials and result in radioactive species that then give off ionizing radiation (primarily beta particles and gamma rays) as they decay. This may include very small quantities of fission products produced from depleted uranium, which is sometimes used in ignition-type targets.

In addition to the prompt (instantaneous) neutron radiation field produced by the DT fusion reaction, a longer-lived but much lower level radiation field is generated by the neutron activation products. The materials in and around the target chamber were judiciously chosen to minimize these induced radiation fields, and protocols such as "stay-out times" were adopted to allow the radiation to decrease to acceptable levels to ensure the worker safety. The NIF radiological goal is, consistent with DOE rules, to limit the annual dose to personnel and individuals in the occupied areas to levels as low as reasonably achievable (ALARA) and well below the maximum yearly dose limits allowed by DOE. Over time, as part of the experimental program, NIF may use other radioactive or hazardous materials during routine operations. Thus it is imperative that NIF put in place the necessary safety systems to manage these hazards and protect workers, the public, and the environment.

\section{A. Identifying and Analyzing Hazards}

\section{Safety Documents}

The hazards associated with NIF and its operation have been identified and evaluated since the earliest stages of design, and safety features have been incorporated into the design to mitigate these hazards. The bounds of NIF operations are described in the National Environmental Policy Act (NEPA) documentation: Final Site-wide Environmental Impact Statement for Continued Operation of Lawrence Livermore National Laboratory and Supplemental Stockpile Stewardship and Management Programmatic Environmental Impact Statement [1] and the Supplemental Analysis of the 2005 Final Sitewide Environmental Impact Statement for Continued Operation of Lawrence Livermore National Laboratory [2]. This NEPA documentation ensures that a thorough evaluation of the impacts of NIF operations has been completed, and that the risks to the public and the environment are understood and communicated. These evaluations have resulted in high level limitations on NIF operations, namely the annual yield (1200 MJ/yr), the annual airborne tritium release $(80 \mathrm{Ci} / \mathrm{yr})$, the maximum individual shot yield (45 MJ), and material inventories (e.g., tritium inventory limited to $8000 \mathrm{Ci}$ ).

The limits specified in the NEPA documentation are flowed into NIF's SBD [3]. The SBD provides a more detailed identification and assessment of hazards, resulting in additional controls to ensure that risks to co-located workers and the public are low. In addition to flowing down yield and inventory limits, the SBD has identified a set of credited safety systems (e.g., radiation shielding) and other credited administrative controls that govern NIF operations. Inventory limits and yield control are implemented through the Facility Safety Plan [4]; Operational Safety Plan (OSP) 581.11, NIF Laser System Installation, Commissioning, and Operation [5]; NIF CIS Radiological Inventory Management System [6]; and other procedures. Credited safety systems are described in more detail in Section b. 


\section{National Ignition Campaign Program Completion Report}

Configuration Management of these systems is critical to ensure continued functionality at the level assumed in the SBD.

\section{Radiation Modeling}

Analysis of potential hazards begins with modeling. A set of computational tools [7] was developed during NIC by NIF's Radiation Safety Analysis Group to help estimate and minimize potential radiation exposure to workers from material activation in the NIF. Prompt doses have been estimated using Monte-Carlo NParticle (MCNP), the industry standard LANL-developed code and a very detailed three-dimensional model of the facility. The radiation analysis model includes all penetrations in both the target bay and the switchyard walls that could allow radiation streaming from the target bay/switchyards to reach occupied areas or the outside of the facility (see Figure 7-1). Initial calculations provided the basis for the facility wall thickness as well as the shield door

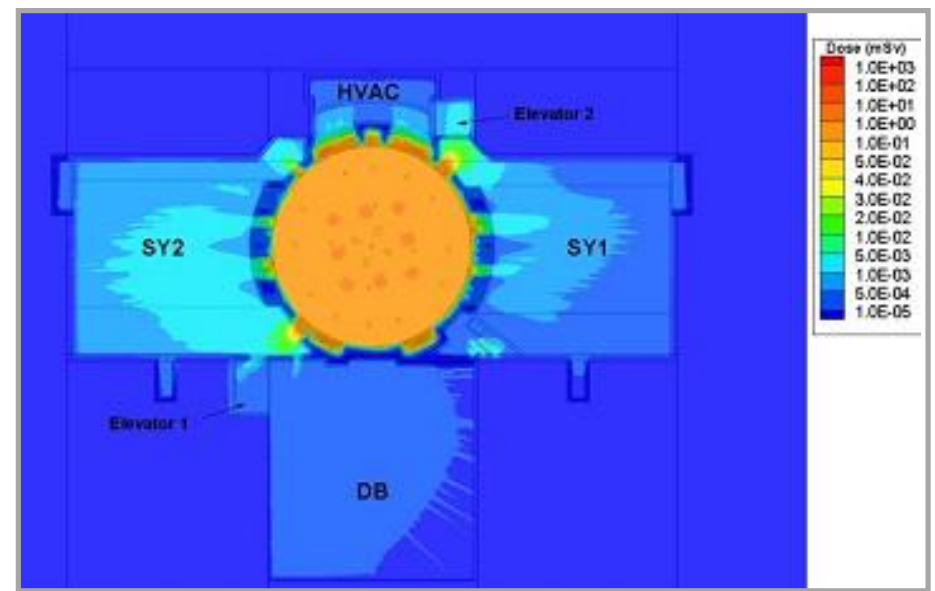

Figure 7-1. High-resolution models of the NIF facility are used to perform both prompt (radiation dose created during a shot) and post-shot (radiation due to short-term residual activation) calculations for estimating radiation levels in the facility.

construction, and the predictions are being validated through a dose monitoring plan.

The Automated ALARA MCNP Interface (AAMI) provides an efficient, automated mechanism for performing the series of calculations required to create dose rate maps (from decay radiation) for the entire facility with minimal manual user input. The NIF Exposure Estimation Tool (NEET) is a web application that combines the information computed by AAMI with a given shot schedule to compute and display the dose rate maps as a function of time. AAMI and NEET are used as work planning tools to determine stay-out times for workers following a given shot or set of shots, and to help in estimating integrated doses associated with performing various maintenance activities inside the target bay.

\section{B. Safety Systems and Worker Protection}

NIF's primary method for controlling hazards is through engineered controls. A set of safety systems has been established as the primary mitigation. These systems are discussed below. Administrative controls (discussed later) may be specified in safety documentation. The NIF work control process ensures that these controls are flowed into work documents.

\section{Radiation Shielding}

Neutrons generated by fusion reactions create prompt radiation that is effectively managed through shielding of the target bay and switchyards. Elements of the radiation shielding system, designed to protect facility workers, co-located workers, and the public from the radiation hazards generated during NIF operations, include the target chamber and its gunite shielding, the target bay and switchyard walls, doors, and floors. The typical thickness of a concrete target bay wall is 6', while the switchyard wall is 3'-3". Shield doors range in thickness from 1' to 6' (see Figure 7-2). 


\section{National Ignition Campaign Program Completion Report}
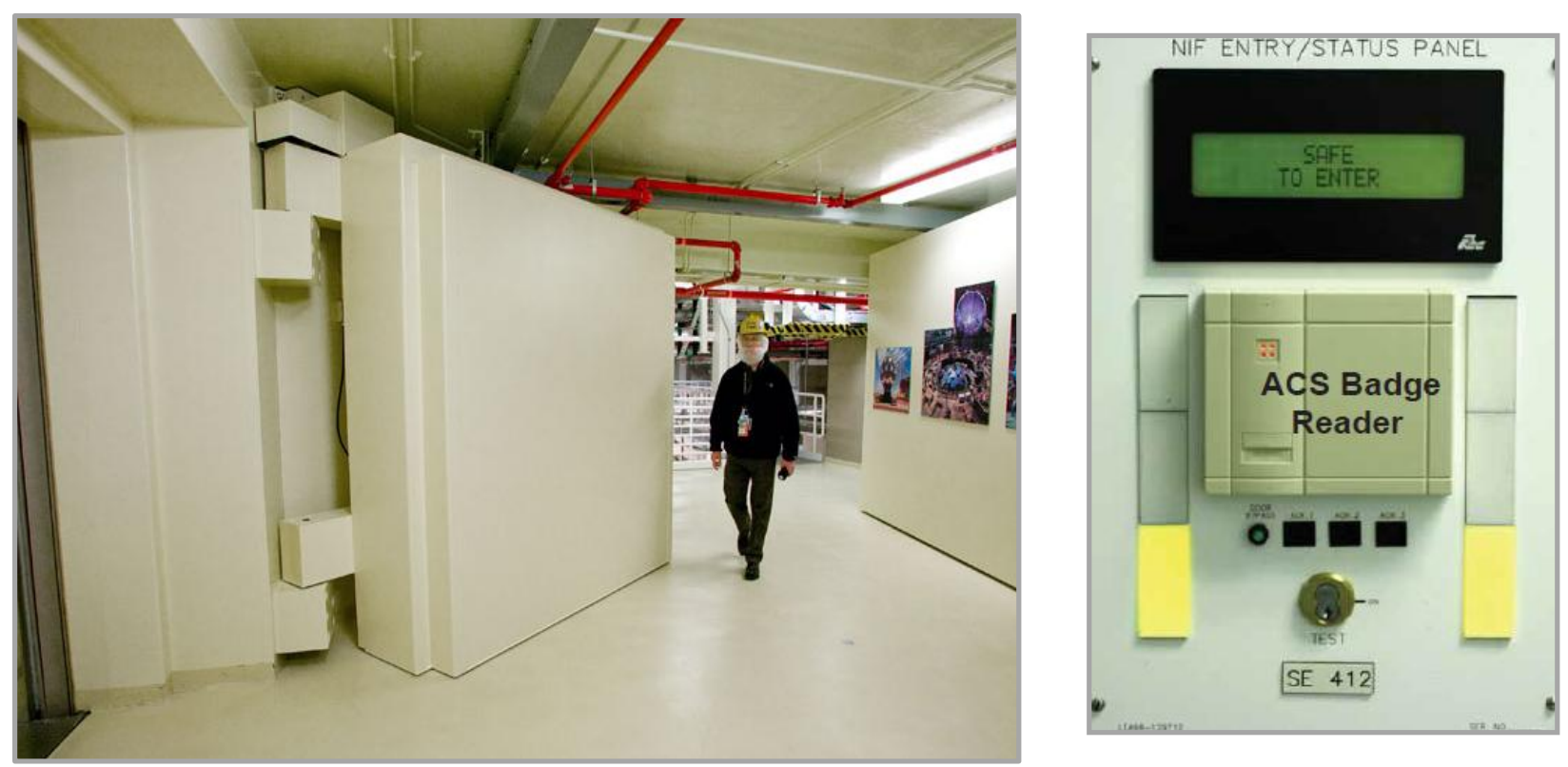

Figure 7-2. (Left) Completion of shield doors and other shielding elements helped enable NIC to ramp up shot neutron yields. Shown above is one of the 44 shield doors installed throughout the NIF. (Right) Safety interlock system entry panel and access control system badge reader used to control facility access.

\section{Safety Interlock System}

SIS, implemented under NIC, works in conjunction with administratively controlled procedures to protect personnel from exposure to high voltage, laser light, radiation, asphyxiation, and other hazards, and where feasible, minimizes equipment damage in the event of a failure in a monitored component in the NIF. SIS provides permissive signals for the operation of process power supplies, alignment lasers, and other devices and monitors the status of safety-related elements in each area of the facility, including shutters, doors (including shield doors), crash buttons, and oxygen levels. It does not control any process devices, but provides a permissive signal for each device interlocked by the system. If the interlock chain for a device is not satisfied, the permissive signal will not be enabled, operation of the device will not be permitted, and it will stay in its fail-safe state or off position. If the interlock chain for a device is satisfied, the permissive signal will be enabled, and operation of the device will be allowed.

SIS functions together with the Access Control System (ACS) to control facility access. ACS tracks entries into and exits from NIF and is used by operations staff to tell who is where in the facility. SIS has been operational 24/7 for over 10 years with no fail-to-danger faults observed. SIS is also discussed in I.8, Operational Capabilities.

\section{Ventilation System}

The ventilation system in the target area is designed to provide air flows and pressures with the intent of maintaining a sufficiently large differential pressure to ensure that, in the unlikely event of a radioactive release in the target bay, the hazardous material would not spread to uncontrolled areas of the facility. Exhaust air and contaminants would not leave the target bay except by means of the target bay exhaust riser, thus being measured by the stack monitor. A similar requirement for the same reason is imposed on the HMMA in the lower level of the Operations Support Building (the maintenance area for contaminated items and location of the TPS, discussed below). These negative pressures also provide some worker protection function since, in the event of a release, the flow of contaminants to other areas would be minimized, if not stopped completely.

The system operates in "confinement mode" on yield shots with expected yield of greater than or equal to $10^{16}$ neutrons. Confinement mode is the simultaneous achievement of requirements of target bay space pressure $(-0.02 \mathrm{WC}$ relative to surrounding areas and the environment) and target bay riser flow rate 


\section{National Ignition Campaign Program Completion Report}

$(<1 \%$ of $\mathrm{TB} \mathrm{vol} / \mathrm{min})$. Limiting the flow rate from the riser will allow for the partial decay of activated airborne isotopes (e.g., ${ }^{13} \mathrm{~N},{ }^{16} \mathrm{~N},{ }^{41} \mathrm{Ar}$ created in the target bay air from shot neutrons). The half-lives of these isotopes are relatively short, requiring that the target bay exhaust riser flow rate be limited for only a relatively brief time (for two hours or less).

\section{Confinement Envelope System}

During the course of NIF operations, targets and target diagnostics may generate a number of hazardous and radioactive contaminants in the target chamber and associated systems. The confinement envelope configured system consists of components belonging to numerous subsystems within the facility that combine to provide the first line of protection against the uncontrolled release of these contaminants into the occupied areas of the NIF. The confinement envelope is not a single stand-alone system, but performs the vacuum or pressure boundary function of components in a large number of subsystems that are connected to the target chamber and have the potential for migration of contaminants from the target chamber. These components, by virtue of their boundary function, act to "confine" hazardous and radioactive contaminants and prevent release to the adjacent occupied spaces of the NIF.

NIF systems that are exposed to target chamber contaminants are well-isolated as part of the engineered confinement envelope. Prior to access, components are isolated from the target chamber by large gate valves and then ventilated. Contamination Zones are small work areas established to manage surface contamination where the confinement envelope is breached, e.g., to remove a diagnostic component. Buffer Zones are established around the Contamination Zones. Although no contamination is expected in Buffer Zones, there is increased monitoring and worker diligence as compared to non-radiological areas. Special permits and radiological worker qualifications are necessary to enter and work in Contamination Zones and Buffer Zones.

\section{Contamination Control System}

The vast majority of NIF's removable radioactivity (primarily surface contamination or gaseous radioactive species such a tritium) is tightly contained in the target chamber and in connected support systems. Post-shot, a small fraction of the target tritium remains on target chamber surfaces and entrant components, such as target and diagnostic positioning systems. The surfaces of these components are identified as "contaminated" and appropriate measures for contamination control are employed when contact with these surfaces is necessary (e.g., removal of diagnostic media; target replacement; diagnostic replacement). Contamination controls at NIF are achieved primarily through the use of installed engineering controls, isolation of impacted areas, personnel protective equipment (PPE), and appropriate worker practices (see Figure 7-3).

The contamination control system configured system receives contaminated gas streams and equipment from the confinement envelope and confines and processes the contaminants. The contamination control system is a set of subsystems that manage contamination from hazardous materials resulting from NIF shots. The system performs three general functions:

- Confinement of contaminants to prevent exposure;

- Mitigation of contaminants by filtration, adsorption, washing, and air purging; and

- Controlled discharge of air and wash waters with residual contaminants to the NIF ventilation stacks and sanitary sewer.

Contamination control system piping includes vacuum pump exhaust piping that is routed to the TPS or to the stack. Contamination control piping is also used to confine target bay air used to flush diagnostic and positioner vessels to reduce residual tritium levels. The contamination control system also includes enclosures: room-within-a-room enclosures that provide additional confinement of contamination, fume hoods for handling and storing contaminated material, and a number of specialized containers, including cabinets for purging optics of residual tritium, transport carts for moving diagnostics from the target chamber to refurbishment areas in the diagnostic building, and containers for transporting tritium gas and tritium-containing targets to and within NIF. 


\section{National Ignition Campaign Program Completion Report}

The confinement envelope and the contamination control systems share a common function to provide confinement of contamination until contamination levels are reduced to negligible levels. In general, the confinement envelope is operated at vacuum; the contamination control systems are generally operated at atmospheric pressure.

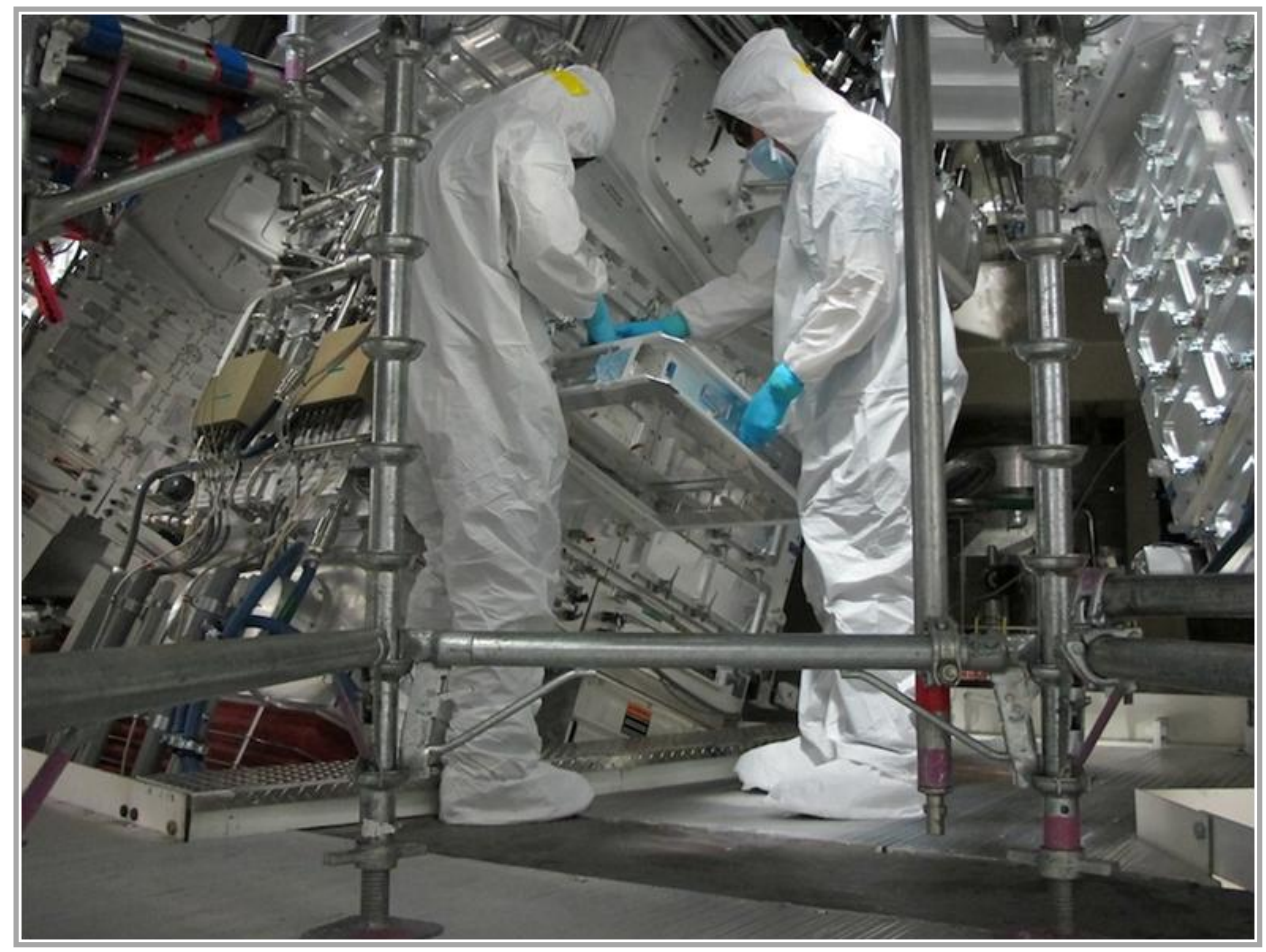

Figure 7-3. Two facility workers in personnel protective equipment are exchanging a potentially contaminated optic from the Final Optics Assembly attached to the NIF target chamber.

\section{Radiation and Hazardous Material Monitoring}

All individuals on site wear radiation monitoring systems (dosimeters) that are read periodically.

Radiation workers involved in tasks with a higher potential for radiation exposure may also be required to wear real-time dosimeters that allow them and their supervisors to track radiation exposure in real time.

Contamination surveys are conducted as part of specific work activities and routinely throughout the facility to ensure that contaminants remain within the specified controlled areas. Portable radiation survey meters (of various types) are used by radiological controls technicians and radiation workers to verify radiological conditions prior to and during work on impacted systems. Standard surface swipes are taken and measured in Liquid Scintillation Counters to determine contamination levels of tritium and other radionuclides. In addition, swipes may be taken to determine the level of beryllium contamination on surfaces and objects.

Permanently installed radiological monitoring systems are used to measure airborne radioactivity and general area radiation levels in the facility. These systems collectively make up the Radiation Monitoring System. Radiation monitoring is accomplished by both direct reading instruments, and by sample collection devices (commonly air and particulate filters) that are periodically removed for laboratory analysis. The Monitoring and Alarm System interfaces with the radiation monitoring system providing alarms when allowable thresholds are exceeded.

The Radiation Monitoring System is divided into four systems, each of which consists of industrystandard radiation monitoring systems configured for the specific identified hazard (x-ray, gamma ray, and tritium). 


\section{National Ignition Campaign Program Completion Report}

\section{a. Stack Monitoring System}

Only a small fraction of the tritium is consumed in fusion reactions. The majority of the remaining tritium is captured by the TPS (see Figure 7-4). Gases expected to contain high concentrations of tritium are first passed through the TPS and charcoal filters, and all gases released through the NIF stack first pass through a HEPA filter. These three components-TPS, stack HEPA filter, and the activated carbon filter-are designed to remove the majority of radioactivity from the air before it is exhausted to the environment (with the exception of activated target bay air). The Stack Monitoring System measures stack ventilation radioactivity to verify the function of these controls and to quantify the amount of airborne activity released to the environment. The system measures

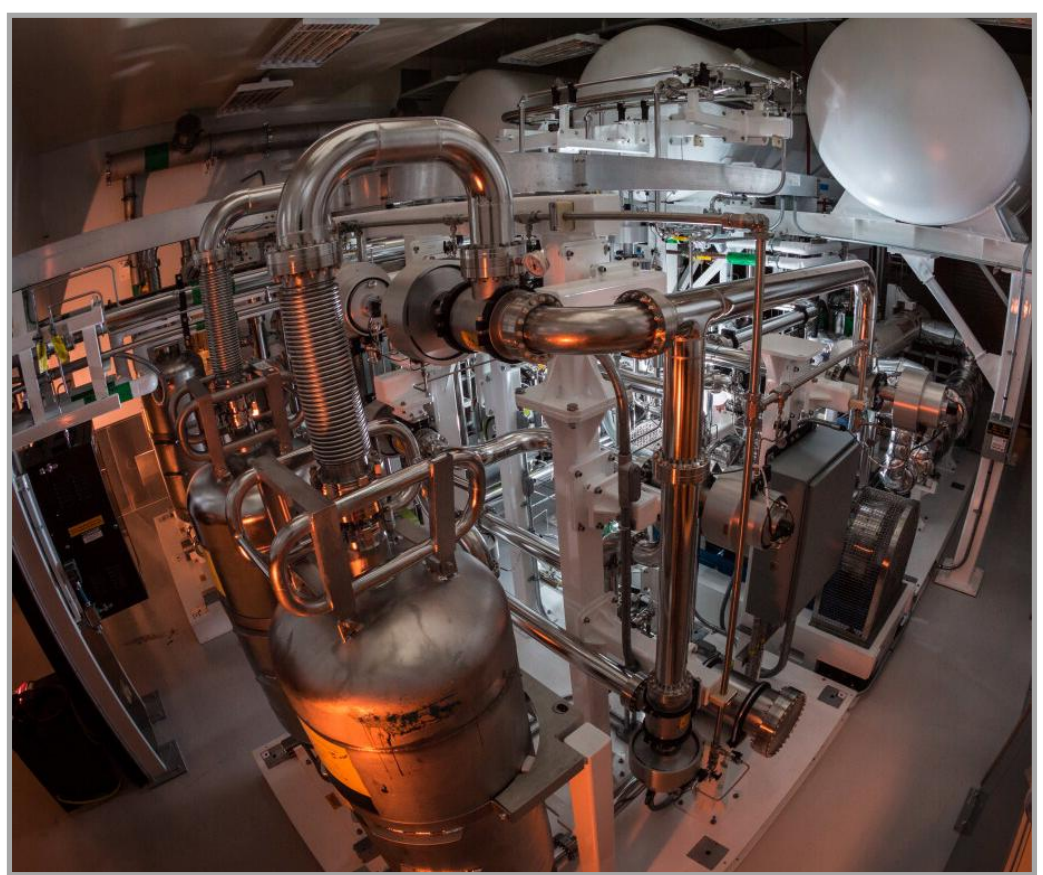

Figure 7-4. Tritium Processing System, with two molecular sieves shown in the foreground. gaseous tritium, radioactive particulates, and radioiodines by collective radionuclides from the stack exhaust on appropriate media that are then analyzed periodically (e.g., weekly) by the LLNL Environmental Functional Area.

Tritium collection is accomplished through a vent and pump process: after a shot, the target chamber or attached systems (sitting at vacuum) that require access are allowed to fill with air. The hydrogen in the air moisture exchanges with the tritium on the vessel surfaces, effectively removing it as the vessel is pumped back down to vacuum. The chamber exhaust is directed through HEPA and activated carbon filters, removing any particulates or other byproduct gasses. Any residual tritium gas is then converted to tritiated water and collected and stored on a molecular sieve bed. The exhaust air, now cleaned of all contaminants, is released, while the potential contaminants remain on filters and adsorbents. These filters, adsorbents, and molecular sieves are subsequently managed as radioactive wastes.

\section{b. Real-Time Tritium Monitoring System}

The real-time tritium monitoring system (TMS) consists of flow-through, gamma-compensated ion chambers designed to monitor the real-time tritium concentration of the stack exhaust and radiological areas of the facility where significant tritium work occurs. The monitor remotely alarms via the SIS system so that operators can take action to minimize worker dose and environmental release (both a worker safety and environmental protection function) in case of an unexpected release of tritium. Although not intended for this purpose (and not a critical function), the TMS also responds to radionuclides other than tritium and can alert operators to large releases of airborne radioactivity.

\section{c. Gamma Area Monitoring System}

The gamma area monitoring (GAM) system is designed to measure the residual gamma radiation levels after yield shots and to continuously monitor radiation levels in the HMMA and RAGS areas so that workers are warned of unexpected high radiation levels. The GAM system is not used to monitor prompt radiation from NIF yield shots, although the detectors outside the target bay are left on during shots. The system consists of two parts: the high-level GAM system, which includes three low-activation ion 


\section{National Ignition Campaign Program Completion Report}

chamber detectors (located in the target bay), and the low-level GAM system, which includes seven tube detectors (located outside of the target bay). While all ten of these detectors are part of the radiation monitoring system, only the HMMA and RAGS monitors perform a critical safety function, as these are the only occupied areas where an unexpected change in radiation levels could occur.

\section{d. CryotARPOS X-Ray Detector System}

The CryoTARPOS load, layering, and characterization x-ray detector system (CTXD) is designed to monitor the general area $\mathrm{x}$-ray dose rate around the CryoTARPOS system during operation of CryoTARPOS LLCS x-ray source. (As mentioned previously, the CTXD is used to characterize the layering process and smoothness of the DT ice layer.) CryoTARPOS is fitted with shielding to reduce local radiation levels to near-background levels during operation. The CTXD system runs continuously during LLCS $\mathrm{x}$-ray operations to ensure that the shielding is successfully performing its function and is interlocked to the SIS to shut down the system in the event that x-ray leakage is detected.

\section{Procedures, Practices, and Protocols}

During NIC, plans, procedures, practices, and protocols flowing from regulatory requirements were put in place to manage tritium, beryllium, depleted uranium, and associated activation and fission products. The required administrative controls were implemented through authorizing documents and work permits for specific activities at NIF. Controls are identified in the Facility Safety Plan [4], OSP 581.11 Appendix L [5], and specific Integration Work Sheet/Safety Plans.

\section{Work Controls}

Every activity at NIF goes through a detailed review and approval process, from the operation of the main NIF laser all the way down to smallest of tasks. The work authorizing document, the integration worksheet (IWS) is where this occurs. Work tasks are evaluated, the associated hazards are analyzed and specific controls for each task are identified. When a more detailed evaluation is required, a safety plan may also be needed. An OSP is an augmentation of the job hazards analysis/IWS and a more detailed ES\&H review of certain hazards associated with a specific activity. An OSP provides a more complete evaluation of hazards and their controls. It also describes likely accident scenarios and the possible consequences if there were no mitigating safety limits or controls in place. Mitigations may include engineering controls (e.g., interlocks, alarms, and shielding), administrative controls (e.g., procedures and signs), and personal protective equipment (e.g., gloves, safety shoes, and respirators). Detailed controls for specific work tasks are flowed down from the safety documents, specified in detail, and approved through the use of a Work Permit, and where applicable, an associated Radiological Work Permit.

OSP 581.11 [5] applies to everyone working in or having unescorted access to the NIF, including Lab employees, contractors, and visiting scientists and engineers. This OSP covers a wide variety of tasks related to laser, radiological, shot operations, and supporting activities. It describes the hazards and control options that can be applied.

\section{Training and Access}

Only knowledgeable and trained workers are authorized to perform work. A detailed training and qualification program was also developed and implemented to ensure that workers understand the hazards and controls associated with their work and are qualified to work safely in the environment at NIF. General, specific, and hands-on training courses for various work levels (Rad Worker 1, Rad Worker 2, etc.) have been established, and radiation and beryllium workers in particular undergo extensive training and qualification.

Radiological barriers/postings may be located in the target bay, switchyards, and Operational Support Building. Special training is required to enter these areas. Training requirements are posted at the area entrances, and personnel are expected to confirm that they meet requirements prior to entry. 


\section{National Ignition Campaign Program Completion Report}

NIF has over 600 qualified radiological workers. These workers are supported by team of radiological control technicians (RCTs), who are specialists in radiological safety. The RCTs are led by the NIF Radiation Safety Officer, who is responsible for implementation of the NIF Radiation Safety Program. The radiological workers and radiological safety professionals ensure that radioactivity at NIF is wellcontrolled and doses from radiation are maintained ALARA.

\section{Sweeps}

As governed by OSP 581.11 [5], the pre-sweep and sweep processes are used in conjunction with SIS and the ACS to ensure that personnel are clear of hazardous areas during the execution of laser shots, target experiments, or other hazardous activities. In addition to the pre-sweep and sweep processes, additional controls are used to ensure that personnel are clear of these areas including warning signs and beacons, warning klaxons, and public address messages.

\section{Personal Protective Equipment}

PPE protocols are an important safety and contamination protection element at the NIF. All PPE must be in good condition and correctly worn. For general facility access and tours, a hard hat is required in the switchyards and target bays. Closed-toe and closed-heel shoes with a non-tapering heel must be worn in the facility at all times. All workers are required to wear appropriate PPE as identified within the applicable IWS/work permit/safe plan of action. This may include a hard hat, safety glasses, and/or other gear. Steel-toed shoes are required if the worker's feet will be vulnerable to injury from sharp protrusions, chemicals on walking surfaces, electrical shock, material handling, and falling or rolling heavy objects. Additional contamination control PPE (coveralls, booties, gloves) may also be required for radiological work.

\section{Facility Readiness}

Facility readiness for hazardous material operations was determined through a series of prestart reviews, performed in accordance with the NIF SBD that describes the suite of hazards associated with planned NIF operations. The Safety Evaluation Report, the mechanism by which the NNSA approved the SBD, identified conditions of approval. These conditions included requirements to perform Management Prestart Reviews (MPRs) ${ }^{\mathrm{ff}}$ prior to the introduction of beryllium, depleted uranium, tritium, and low-yield operations $\left(<1 \times 10^{16}\right.$ neutrons/shot), and to conduct a contractor Readiness Assessment (RA) ${ }^{\mathrm{gg}}$ prior to ignition operations with shots up to $1 \times 10^{19}$ neutrons.

NIF had previously conducted both a Contractor and an NNSA RA for Integrated Facility Operations as part of project completion; these reviews examined readiness for basic facility operations using up to 192 laser beams. The reviews for hazardous materials introduction and yield generation built upon those early reviews and focused specifically upon those preparations necessary for advancing into hazardous material and yield operations. These focused performance-based reviews evaluated NIF's readiness to proceed, confirming that: (1) the facility was in a state of readiness to safely conduct the subject operations in accordance with the safety basis; (2) the plans and procedures were in place to ensure that safe operations could be sustained; and (3) personnel were trained and qualified.

The review for tritium introduction was conducted in two parts. First, an MPR was conducted to examine facility readiness to tritium introduction to the CryoTARPOS for layering only. This review occurred in May 2010 [9]. A second MPR was conducted to examine readiness to shoot tritium-containing targets.

ff A MPR is an internal NIF process for examining equipment, personnel, and plans and procedures and evaluating readiness to proceed with a prescribed scope of work.

${ }^{\text {gg }}$ Although a RA is very similar in nature to a MPR, it is much more rigorous and conducted by an independent panel of experts (members are generally from outside LLNL). 


\section{National Ignition Campaign Program Completion Report}

The scope of this second MPR also included low-yield operations

$\left(<1 \times 10^{16}\right.$ neutrons/shot $)$ and the use of beryllium and depleted uranium. The MPR for beryllium, DU, tritium, and low-yield operations [10] was conducted in July 2010. In both cases, the MPR Committees recommended that the NIF Authorizing Individual grant conditional authorization to proceed with the scope of work under review once the prestart findings were resolved. The prestart findings from both reviews related to the introduction of tritium, DU, and low-yield experiments were closed with concurrence from the DOE/NNSA NIF Project Division, enabling these operations to commence on September 3, 2010. The program has not yet required that beryllium be introduced. One prestart item related to the use of beryllium remains open: performance of dry runs. This item will be closed just before beryllium introduction is required.

After authorization, tritium was injected into the target chamber as part of the tritium handling performance qualification test. Tritium gas was injected from a manifold containing five bottles of 100 $\mathrm{mCi}$ each. All systems behaved as expected. A second injection of tritium followed with the same successful result. This introduction of tritium into the facility marked the beginning of operations with hazardous materials. Subsequently, yield-producing target shots with tritium were performed using the hazardous material protocols reviewed during the MPRs.

The second MPR Committee stated in their report that the NIF was on schedule to complete all the facility modifications and controls implementation necessary for ignition operations during September 2010. However, it was the MPR Committee's recommendation that NIF be operated in the regime addressed by the MPR review for a reasonable period of time prior to conducting the contractor RA and initiating ignition operations. As a result of this recommendation, the scheduled completion date for this RA milestone was changed from September 2010 to March 2011 after it was determined this delay would not impact the planned experiments on the path to ignition.

On March 4, 2011, the contractor RA for ignition experiments concluded that facility systems and equipment, training, and management controls were in place for NIF to safely perform experiments with yields of up to $10^{19}$ neutrons. In their report [11], the RA team recommended that the NIF Authorizing Individual grant authorization to proceed with ignition operations once the single prestart finding was closed. This item was closed out and verified by NNSA on May 23, 2011.

\section{References}

1. Final Site-wide Environmental Impact Statement for Continued Operation of Lawrence Livermore National Laboratory and Supplemental Stockpile Stewardship and Management Programmatic Environmental Impact Statement, DOE/EIS-0348, DOE/EIS-0236-S3, March 2005.

2. Supplement Analysis of the 2005 Final Site-wide Environmental Impact Statement for Continued Operation of Lawrence Livermore National Laboratory, DOE/EIS-0348-SA3, August 2011.

3. NIF Safety Basis Document, NIF-5019666.

4. Facility Safety Plan, NIF-5019665.

5. Operational Safety Plan 581.11, NIF Laser System Installation, Commissioning, and Operation, NIF-5017298.

6. NIF CIS Radiological Inventory Management System, NIF-5030388.

7. J. Verbeke et al., "Planning Tools for Estimating Radiation Exposure at the National Ignition Facility," Fusion Science and Technology 60, 595 (2011).

8. R.K. Reed, "Safety Systems and Access Control in the National Ignition Facility," Presentation to 57th HPS Annual meeting/AAHP Special Session, July 24, 2012.

9. Readiness to Introduce Tritium for Cryolayering Management Prestart Review, NIF-0116557.

10. NIF Management Prestart Review-Beryllium, Depleted Uranium, Tritium, and Low Yield Operations in B581, NIF-0116725.

11. National Ignition Facility Ignition Readiness Assessment Final Report, NIF-0117362. 


\section{National Ignition Campaign Program Completion Report}

\section{I.8-OPERATIONAL CAPABILITIES}

The main scope of work conducted under the NIC WBS Element I.8, Operational Capabilities, is to provide planning and the preparations, training, operations, and maintenance of the NIF for NIC. This includes the operations and maintenance of the NIF facility, the laser system, diagnostics, cryogenics, user optics, transport and handling, other operational equipment, and assembly and refurbishment facilities, infrastructure, and equipment. It also includes the ongoing operating inventory and the replacement hardware needed for ongoing maintenance, as well as personnel associated with this effort, including those needed to execute the ignition experimental plan (laser and target area) and other user campaigns. The NIF Shot Operations Plan [1], in conjunction with the NIF Operations Management Plan [2] and NIF Maintenance Plan [3], satisfy the requirements for a Conduct of Operations.

The organization that operates the NIF facility for the NIC campaigns is shown in Figure 8-1.

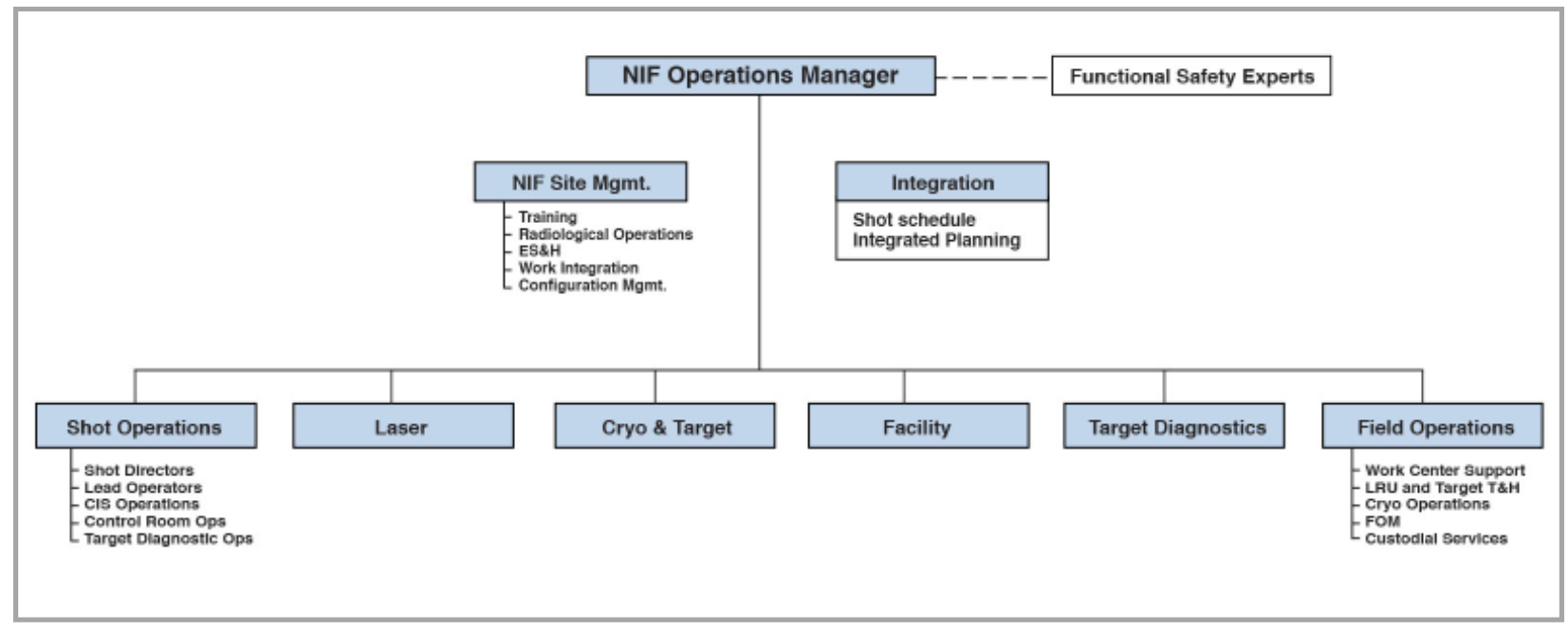

Figure 8-1. NIF Operations Organization.

NIF Operations management has the overall responsibility for the safe, cost-effective, reliable performance of the NIF. Staff assignments include project controls and administration for the organization.

NIF Site Management is responsible for conduct of operations implementation, safety basis compliance, standards and policies, work coordination, configuration management (CM), security, ES\&H, NIF's Safety Program, hazardous material operations, radiological operations, training, and business and staff administration.

Shot Operations is responsible for conducting scheduled shot sequences safely, reliably, and cost effectively. Shot Operations staffs and manages the control room.

Facility Operations and Maintenance operates and maintains the NIF conventional facility and other commissioned systems that support laser system operations, as well as provides maintenance management, calibration services, and logistics support functions.

The Laser, Cryogenic and Target, Facility, and Target Diagnostics organizations are responsible for the maintenance and operation of the technical equipment within the NIF, ensuring that systems are performing safely, cost effectively, and reliably, while meeting technical requirements. They work closely with NIF Engineering and Systems Engineering to ensure that systems meet performance requirements, implement upgrades, and improve availability and reliability. 


\section{National Ignition Campaign Program Completion Report}

\section{A. NIF Shot Operations}

Shot Operations are defined as those activities directly related to or supporting the operation of the NIF Main Laser System, Precision Diagnostic System, target chamber, and associated systems located on the NIF. Operations include delivery of low-power laser light through to the firing of the main laser amplifiers, operation of the beampath and associated utilities, target positioners and diagnostics, and the various computer control systems as well as archival and initial processing of the experimental data.

\section{Shot Planning}

A shot planning process and organization was set up as part of the NIC. The highest level shot planning is performed on a yearly basis by the program leads at the Experimental Facility Commissioning meeting. Once the schedule has been established, the three-month experimental shot sequence is integrated with the facility and capability implementation at the Facility Laser Interactive Planning (FLIP) meeting. On a weekly basis, the FLIP shot schedule in integrated with facility maintenance and calibration activities. This results in an approved set of activities that is then scheduled in detail by the facility work planning organization in a daily meeting in the Detailed Operations Schedule tool. Figure 8-2 shows the roadmap for the shot planning process.

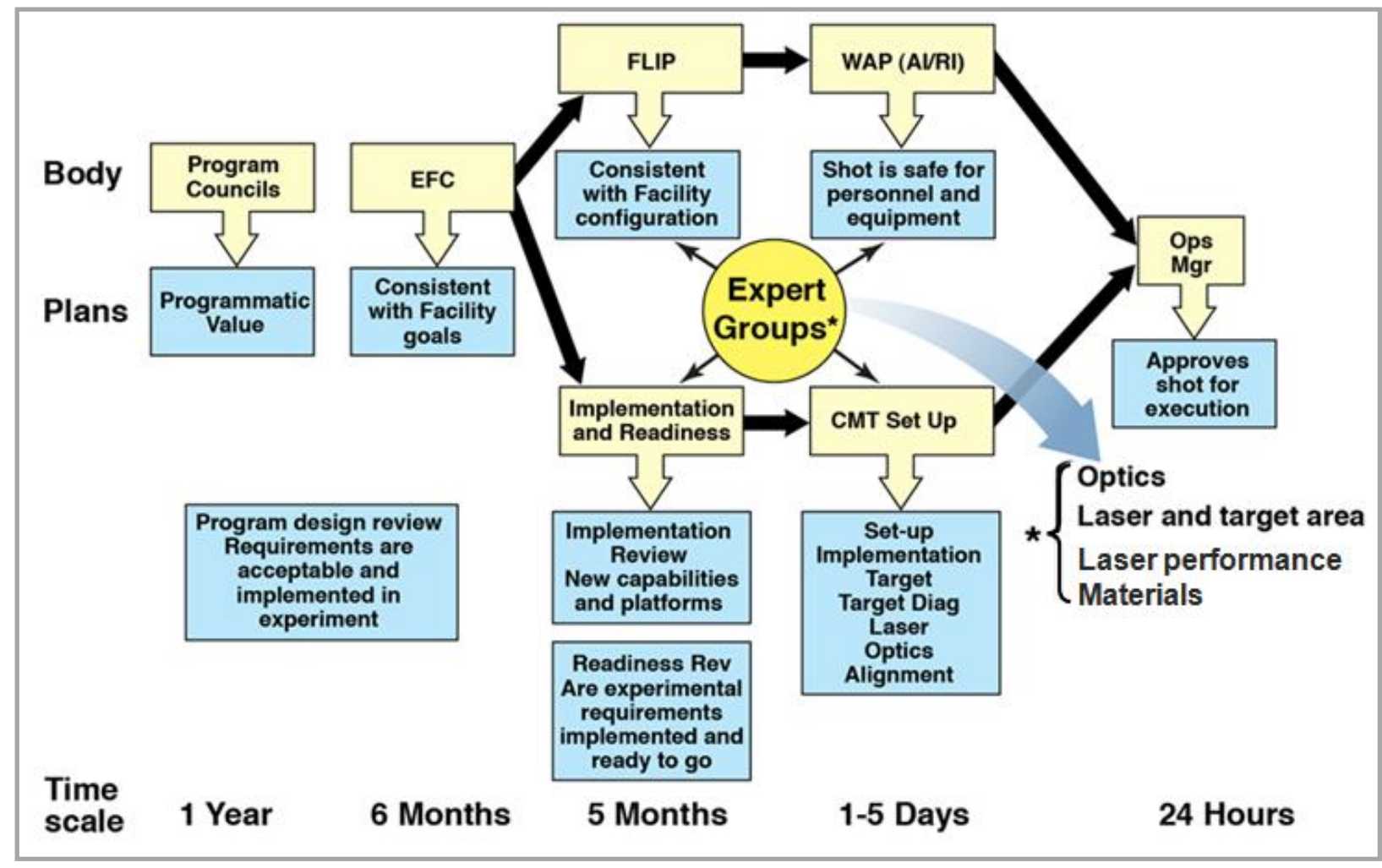

Figure 8-2. NIF's shot planning and review process.

\section{Experimental Roles and Responsibilities}

Roles and responsibilities have been established to ensure the smooth the design, planning, and execution of experimental campaigns. Major roles include the following.

The Campaign Responsible Individual (RI), who is either the Principal Investigator (PI) or Liaison Scientist, oversees execution of the NIF experimental campaign and is responsible for organizing progress meetings; ensuring that the development of the experiment is consistent with the facility, capabilities, and schedule; developing an execution plan; providing regular updates on experimental progress to the Authorizing Individual (AI); and negotiating with the supporting program and NIF staff regarding capabilities and priorities as necessary to facilitate the experiment. 


\section{National Ignition Campaign Program Completion Report}

Each existing shot type or experimental platform has a Platform RI, who is familiar with the relevant configuration/platform and provides platform expertise to the experimental team. The Campaign RI works with the relevant Platform RI. In many cases, the Campaign RI serves as the Platform RI.

A responsible Project Engineer is also designated for each experiment. For experiments using existing diagnostics, a Diagnostic Responsible Scientist (RS) for each applicable instrument works with the RI. The RS is responsible for providing quality data to the RI. This includes ensuring that the instrument operates as planned and acquires data.

The NIF Operations Manager (NOM) is the final approval authority for all NIF experiments.

\section{Shot Execution}

An effective shot design and execution process was established during NIC. Key milestones during the process are the reviews, which are opportunities to gauge experimental readiness and ensure that the plans and capabilities are in place to ensure that the experiment meets program goals. Important steps are described below.

Program Review: The Program Review is led by the AI and is typically conducted approximately six months to one year in advance of the start of the experimental campaign. The primary purpose of the review is to ensure the proposed target design and associated campaign and experimental plan will meet the designated scientific and programmatic objectives. This review may occur more than once as dictated by the needs and progress of the campaign. The sponsoring program determines the agenda for the Program Review.

Campaign Review: This review is performed only for campaigns that are using preexisting capabilities, diagnostics, and platforms. The goal of the review is to identify any major issues that preclude this specific shot sequence in this time frame.

Implementation Review: The Implementation Review is led by internal senior NIF staff familiar with the planned experiment and associated facility issues. The review includes members of the NIF expert groups, FLIP, and key members of the proposal team, and examines all aspects of the detailed plan for experimental execution. This review should occur roughly four months before the experiment; the timing of this review depends on the capability and development needs.

Representatives from each of the NIF expert groups attend this review. Prior to the Implementation Review, the RI should work with the NIF expert groups (see I.2, Systems Engineering) to identify and resolve issues associated with execution of the experiment. This includes consideration of target debris, unconverted light, target manufacturability, laser and user optic specifications, and the like.

In preparation for the Implementation Review, targets with significant engineering issues may require a separate formal engineering design review. This should be arranged by the RI and the NIF target fabrication organization.

Readiness Meeting: This meeting, led by senior NIF staff, occurs approximately one month prior to the date of the experiment and is the final check to ensure that all preparations for execution of the experiment are complete. All specifications for setup of laser, diagnostics, and user optics are finalized at this time.

Experiment approved for execution following Readiness Meeting. Following successful completion of the Readiness Meeting, and input of all necessary setup parameters to the CMT, the experiment will be approved for execution by the NOM.

Shot is executed. The RI will also provide a pre-shot briefing to the Shot Director, and the NIF Operations staff will ensure that all setup sheets are approved. The RI also attends a meeting the morning of the experiment. NIF Operations staff performs the shot briefing and begins the shot countdown. The shot is performed. 


\section{National Ignition Campaign Program Completion Report}

Post-experiment operations review occurs. The day after the experiment, an operations-relevant review is performed to address any issues that arose during the experiment with the laser or diagnostics.

Campaign RI leads post-campaign review for the experiment. This involves a post-shot summary debriefing and data analysis report, listing of lessons learned, and a review or discussion of the data with the Diagnostic RSs.

\section{NIF Expert Groups}

NIF has a number of expert groups that are consulted throughout the shot preparation and execution process. The expert groups formally review experiments at the Implementation Review. Formal expert group approval is obtained via the WAP checklist process. For more on expert groups, see I.2, Systems Engineering.

\section{B. NIF Maintenance}

The reliability of the NIF, including its support systems and utilities, is essential to ensuring that NIF is available to support laser operations. The NIF Maintenance Plan [3] describes the system's equipment and assets, boundaries, interfaces to other systems, and the maintenance approach, including failure modes and general responses for major system off-normal conditions. Included in its scope are the Laser and Target Area Building (B581), Optics Assembly Building (B681), and associated utility pads and outbuildings (B582, B682, B683, and B684).

NIF's maintenance policy is composed of a hierarchy of documents, where the top level provides general policy for maintenance in the form of process maps and work implementation procedures, and the lower tiers provide more specific guidance in the form of maintenance procedures and checklists. The process map below represents the maintenance workflow and basic function of support activities (Figure 8-3). Furthermore, it shows the interrelationship of various processes and subprocesses to provide an organization-wide overview for the purpose of creating commonality in performing maintenance.

The NIF maintenance strategy has been to perform preventative, corrective, and reactive maintenance on all systems as appropriate to best maximize facility availability. Each system is evaluated to ensure the best combination of preventative, corrective, and reactive maintenance is applied to maximize system shots. This maintenance strategy has worked well because predictive and condition-based (vibration monitoring, thermal imaging, oil sampling, etc.) maintenance strategies had been added for many systems, where the maintenance can be scheduled and performed between shots as much as possible.

The goal of the NIF Maintenance program is to achieve a reliable, available, and maintainable (RAM) facility [5] and RAM support systems at the highest efficiency, quality, and safety standards. A reliabilitycentered maintenance (RCM) program was recently deployed to reduce failure rates and the time required to repair equipment, anticipate problems before they occur, respond faster to failures (by having parts, permits, and procedures ready), and plan for windows of opportunity. Using the RCM process to decide where to focus maintenance resources has allowed NIF to:

- Identify and focus on shot-critical functions.

- Determine critical failure modes and impacts.

- Strategically apply health monitoring tools to anticipate problems.

- Evaluate the most cost effective mitigation to preserve functions.

- Tailor tasks based on impact to shots. 
National Ignition Campaign Program Completion Report

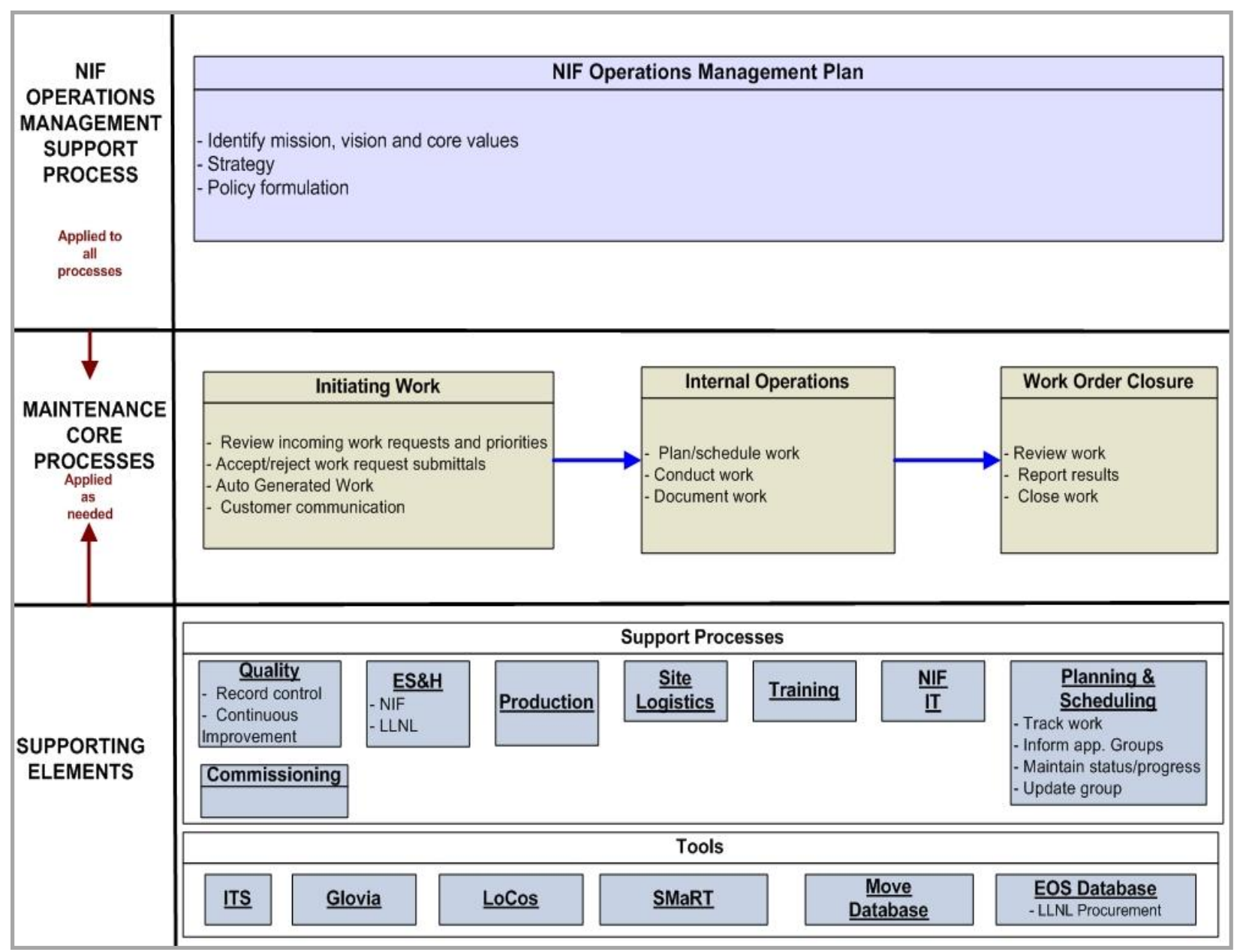

Figure 8-3. Maintenance process map.

\section{Spares}

Adequate spares are available for maintenance equipment such as forklifts, transporters, and cranes. For the laser systems, initial spares have been defined and are continuously updated based on operational failure rates; NIF aims have at least one full spare for every line-replaceable unit. NIF facility availability has not been impacted by spares unavailability during any of the previous years of operation. The process for determining spares involves incorporating modeling results based on current operational experience of failure rates and recovery times. Optics production has been calculated based on the shot plan.

For conventional facilities and utilities, spares are set based on the RCM analysis. For these systems, often there is a large degree of redundancy. In these cases, spares are minimized and replacements are ordered upon failure. Exceptions to this rule are items with a very long lead time or items that have a very large impact to shot operations (for example, vacuum pumps).

\section{Maintenance Periods}

To better plan and coordinate maintenance activities with shot activities, NIF has defined the following maintenance periods:

- Type 1: Maintenance activities that are integrated with shot operations with the work usually completed in one twelve-hour shift.

- Type 2: Maintenance activities that can be completed in less than six twelve-hour shifts (less than three days) with some effects on shot operations, depending on details of the specific activity. 


\section{National Ignition Campaign Program Completion Report}

- Type 3: Maintenance activities that takes longer than six twelve-hour shifts (more than three days) to complete significant facility maintenance and reconfiguration activities. During Type 3 activities, shot operations will be significantly affected.

The maintenance periods are coordinated with Shot Operations starting with the high level shot plan. The maintenance activities fully integrated into the daily operations schedule, and potential conflicts among maintenance activities and shots are de-conflicted at twice-daily plan of the day meetings - one for day shift and one for night shift.

\section{Maintenance Plans and Procedures}

System-level maintenance plans (SLMPs) detail the approach and methods designed specifically for each of NIF's approximately 160 systems. The SLMPs are developed based on RCM principles, with a focus on maintaining functional requirements for each system. The RCM process begins with a system functions determination and then proceeds with a failure modes and effects analysis (FMEA) to determine critical failure modes and their impact on shots; it concludes with a determination of the most costeffective mitigation to preserve functions. Results from the RCM process lead to a tailored set of tasks based on the system's importance to shot functions.

A configured system (CS) is a system that performs a safety or environmental preservation function. That is, the equipment and assets contained within the CS perform a specific safety function or meet requirements defined in the Site-Wide Environmental Impact Statement [6]. The specific maintenance required to maintain the functionality of the CS is included in a CS maintenance plan. The primary purpose of this plan is to document the critical functions and the methods of ensuring their performance.

In nearly all instances, procedures are required when performing work on NIF's systems and are provided for all maintenance being performed under the following conditions:

- High consequence of failure: When failure to correctly perform a specific sequence of steps for an activity would likely result in high consequences to the project, environment, safety, and health.

- Complex work activity: When a work activity is so complex that authorized and qualified workers may not successfully and safely complete it without a procedure. Examples include:

$\circ$ Detailed activities where repeatability or a high degree of quality is critical.

○ Maintenance work on configuration items (CIs).

- Infrequent Performance: When moderately complex activities are not routinely performed.

Information about requirements for, preparing, reviewing, approving, controlling, distributing, revising, and using NIF Operations procedures for operations and maintenance within the NIF Complex, can be found in NIF Procedure 5.14, NIF Procedures and Plans Writers Guide [7].

\section{SMaRT}

The Systems Maintenance and Reliability Tracking tool (SMaRT) is the computerized maintenance management system based on the INFOR product that contains information to enable the processes necessary to perform maintenance on NIF subsystems. SMaRT tracks work that is performed and retains the records of work performed.

For conventional facility and utility systems, each maintenance activity is described by a work order. Each work order proceeds through a review and approval process prior to being executed in the field. Typically each work order contains a maintenance procedure detailing the work activity. In addition, when material is required to perform the activities, the work order contains a material list. This material list is tied to our inventory management tool, Glovia, to facilitate ordering, storage, and kitting of required parts. 


\section{National Ignition Campaign Program Completion Report}

Each work order undergoes a close-out review where data from the field and booked hours are captured. In addition, various data fields are set or completed during the close-out process to enable sorting of data for RAM analysis or other metrics.

\section{Maintenance Organization}

The NIF organization assigns responsibility for various systems to a system manager. A system manager typically is responsible for several like systems. The system manager is responsible for:

- Understanding all safety aspects related to a particular system.

- Creating the SLMP that defines the maintenance required for that system.

- Directing the work by first defining the work through maintenance procedures and work orders.

Often the system manager will be in the field to guide the technicians; this is especially true for first-time activities, activities with a high level of consequence, or activities that happen rarely. Because the system manager's responsibilities span multiple systems, often a system manager will have a helper. The helper is typically an engineering associate, while the system manager is a degreed engineer.

The system managers are supported by a team of trained technicians. The technicians work out a pool managed by the work center supervisor. Typically, the technician team is split into multiple shifts in order to support the 24/7 operations of the NIF. The system manager works with the department planner to schedule the work activities by deciding in which week to "bucket" work. Then the department planner and the work center supervisor create a detailed weekly schedule assigning technicians by name to the activities.

There is also a small integration group that assists all system managers. The integration group owns the SMaRT tool; SMaRT administrators in the integration group assist the system managers in setting up their preventative maintenance program in SMaRT. The integration group is also home to condition-based maintenance tools such as vibration analysis, thermography, oil sampling, and ultrasonic monitoring. As it is not practical to have each system manager to become proficient in all of these tools, the expertise resides in the integration group and is a resource for all system managers. Finally, calibration, another activity that cuts across all systems, is part of the integration group; NIF's calibration manager is part of the integration team.

\section{NIF Site Management}

\section{Site Management Mission}

The NIF Site Management organization's mission is to provide a "one-stop shop" for NIF, integrating all staff support functions to allow the maintenance, operations and commissioning activities to proceed safely, securely, and in a coordinated manner. This includes defining standards and policies, authorizing and coordinating work activities, developing and implementing configuration management, and planning and implementing a training program. The organization also provides facility management to ensure continued compliance with LLNL ES\&H, NIF directorate policies, and integration services for all work activities, tours, events, and other access needs. In addition, the Integration organization establishes and implements standards for training, worker behavior, cleanliness, safety, and security.

\section{Training}

The NIF Operations training philosophy is to maintain a standardized, proactive training posture to develop and expand the level of expertise of the workforce and to establish uniform standards of safe operation. The training program must be consistent, efficient, and responsive to the dynamic skill and training requirements for meeting programmatic goals and objectives.

Determination of the rigor of training required for any given job or task is based on three items:

- Risk-based analysis of the position or task by Subject Matter Expert, approved by Operations management. 


\section{National Ignition Campaign Program Completion Report}

- Current skill set of person performing job or task.

- Availability of existing materials, procedures, and qualified trainers.

Training methodology may consist of web-based classes, instructor-led classes, or on-the-job training, often referred to as Qualification Cards. All training course completions are tracked in LTRAIN.

\section{Configuration Management}

Configuration Management is an integrated management system designed to maintain the relationship between requirements, data, execution, and the physical/functional configurations. This involves the systematic identification of NIF configurations and the management of changes to those configurations. The NIF configuration is defined as the as-built, tested, and verified NIF, facilities, and process equipment delivered by the NIF project and accepted for NIF operations. The term "configuration" encompasses not only the physical items delivered but also the controlled safety and performance requirements and criteria that those items have been verified to satisfy.

CIs are items within CSs that have been identified as needing additional controls in order to ensure the system is able to perform its intended performance or safety function. A CI list is generated for each CS by the Functional Safety Expert (FSE), which is reviewed and approved by the FMEA Working Group and subsequently entered into ECMS. CIs can specifically be hardware, software, design requirements, procedures, programs, and documentation. Controls have been established to ensure that physical and design configurations are maintained as required per the NIF\&PS Configuration Management Plan [8].

The functionality of the CS is overseen by the FSE. Any work that affects the performance of CIs must subsequently be reviewed and approved by the FSE. If the work will affect the ability of the system to perform its safety functions, impairment controls must be put in place. The system manager is responsible for ensuring that the physical configuration and functionality of the CIs are maintained. New systems or additions to existing systems will be evaluated for new CIs and impacts to CSs using the existing WAP and Work Control processes.

The NIF CM process is applied in a graded manner. Those elements related to public safety, worker safety, the environment, significant programmatic impact, and Safety Basis administrative controls are CIs and undergo more rigorous review than those that support functional requirements and facility functions.

\section{Off-Normal Preparedness, Response, and Notification}

The NIF and Photon Science Directorate What to do in an emergency [9] brochure provides basic emergency response actions. The Off-Normal Event and Notification Process [10] reiterates the basic emergency response actions and provides notification instructions following an emergency.

Reactions to major emergencies are coordinated and managed by the directorate ES\&H organization. In the event of a major emergency, such as an earthquake or fire, personnel will be directed to leave the building and proceed to the facility assembly point, per the NIF Emergency Preparedness and Response Plan [11]. Annual evacuation drills are conducted for all work shifts in order to reinforce emergency response actions.

A NIF Operations phone list is maintained for key personnel, including work, home, and cell phones.

\section{Work Control}

Work that is executed on the NIF has to be planned and coordinated. Proper work planning and authorization are crucial for achieving safety. Work planning requires: 


\section{National Ignition Campaign Program Completion Report}

1. Developing the detailed scope of work, schedule, and interfaces;

2. Analyzing the associated hazards; and

3. Developing a plan to mitigate those hazards.

The NIF has a challenging set of work activities and operations requiring a higher level of coordination. Work must be authorized before it can proceed, and the work area and activity must be reviewed before the start of each work task. The initiating steps for work planning and authorization begin with the Job Hazard Analysis (JHA)/IWS/OSP. This process ensures that the work is properly planned and authorized. The organization authorizing a work activity is responsible for ensuring that a JHA/IWS is prepared, reviewed, and approved prior to performing any work; refer to the NIF\&PS Directorate Safety Manual [12].

A WAP or MPR may be conducted in certain instances prior to the AI approving the IWS/JHA. An MPR is required if a significant new hazard is introduced, as described in Safety and Performance Review Board, Management Prestart Reviews, and Working Group Reviews [13]. WAPs are used whenever an activity is being performed for the first time, when significant changes to the processes involved have occurred, when the work has the potential to affect the facility safety basis, or when requested by the AI. Refer to the Work Authorization Review Procedure [14] for specific details.

Work performed at the NIF is managed and controlled by a work permit process [15]. Work Permits specifying scope of work, and associated hazards and their controls, are initiated by the Work Permit Responsible Individual (WPRI) and approved by the Work Permit Approver. Prior to commencing work, the WPRI submits the Work Permit to the Work Control Officer at the Work Control Center for review, coordination, and integration with other work activities and priorities and for final release. LoCoS is used to create, manage, and archive work permits.

\section{Work Planning and Execution}

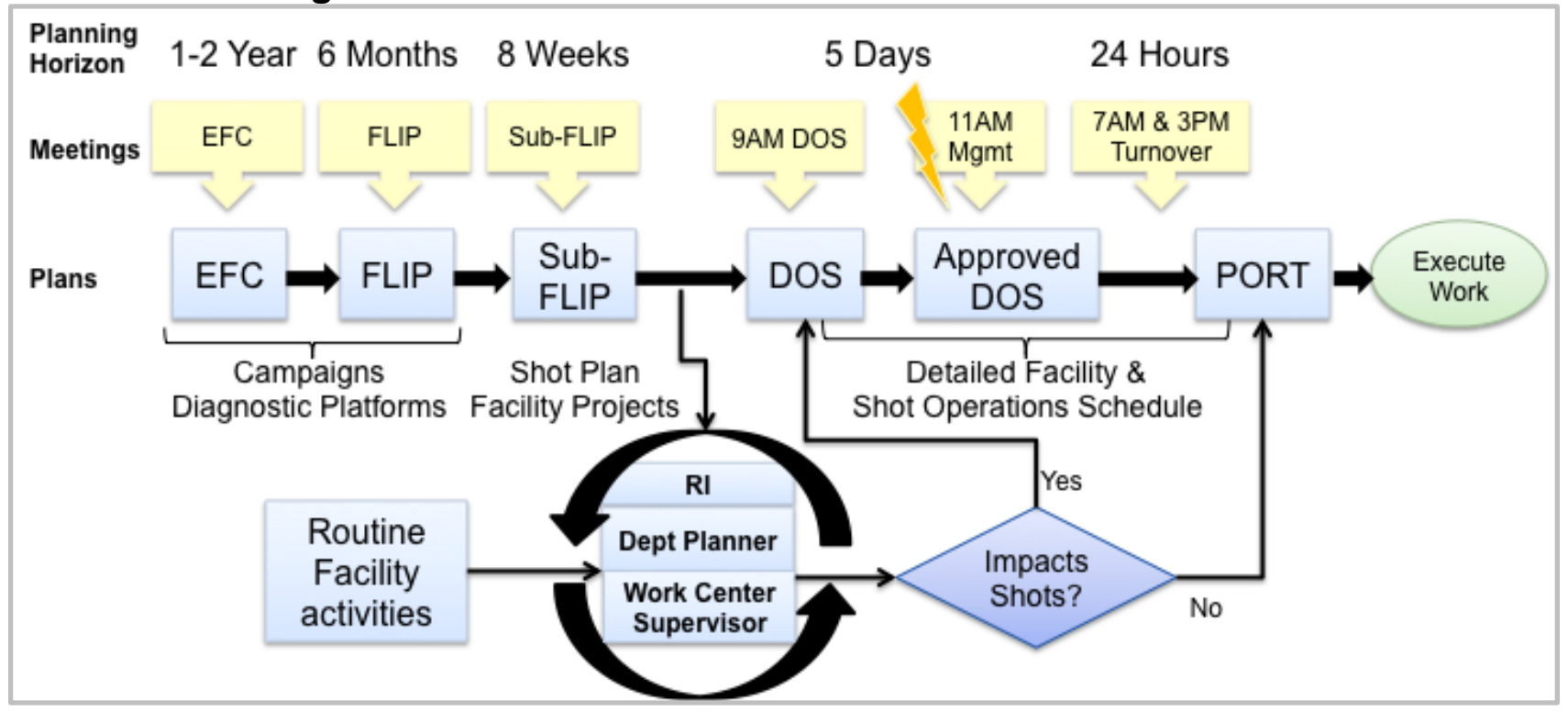

Figure 8-4. NIF maintenance planning process is integrated with the shot scheduling.

As shown in Figure 8-4, the work planning and execution process has various time horizons. Near term (five days or less) work planning and execution rely on the department planners working with the work center supervisors to allocate resources and coordinate among the work centers and shot operations to deconflict work. Their roles are described below.

Department Planner: The Department Planner for each area is responsible for understanding the maintenance and other work that needs to be accomplished. The Department Planners gather this information from the SMs and SMaRT, where applicable. They chair the weekly planning meetings and 


\section{National Ignition Campaign Program Completion Report}

work with the SMs to prepare maintenance window packages and present them to the SubFLIP for scheduling. The Work Center Supervisor assists the Department Planner in organizing and tracking progress of the work packages from execution to closeout.

Work Center Supervisors: The Work Center Supervisors know the resources (labor, time, and equipment) required to perform work. They work with department planners to prepare resource-level weekly plans and allocate the labor to support the daily work plan. They are responsible for reviewing work permits for scope, hazards, and controls (serving as WPRI) and ensuring that work is safe, well planned, and ready to proceed.

Field Supervisors: The Field Supervisors are in the field supervising the technician teams assigned to their areas. They work with the Work Center Supervisor to develop daily work plans and make the daily job assignments. They also conduct the shift turnover meeting (prepare report and distribute).

Work Control Office: The function of the Work Control Office is to ensure that all work activities are integrated on a daily basis. This step is essential for establishing that concurrent work activities have been appropriately prioritized and are compatible with other ongoing work and with current facility conditions.

\section{Cleanliness Protocol}

The function of the NIF clean construction protocol program is to ensure a clean work environment exists to produce high-quality optics, optical components, and mechanical systems. Eliminating optics contamination positively impacts the efficiency of NIF operations over the long term. The Clean Protocol Manager leads these efforts by defining, developing, and implementing appropriate cleanliness protocols [16] and associated quality programs for cleanrooms and clean areas in the NIF.

\section{Safety}

NIF's primary method for controlling hazards is through engineered controls. The NIF has identified the safety systems that are required to perform specific functions related to maintaining the safety basis, ensuring worker safety, and providing environmental protection, which are collectively called CSs. They are subject to enhanced configuration management, which ensures that the as-built condition, associated documentation, and requirements are always consistent. These systems and their functions are summarized below. Safety and PEPS are discussed further in I.7, PEPS.

\section{Control of Energy Sources}

A lockout/tagout program is the principal process used by NIF Operations to protect personnel from injury. Through this process, potentially hazardous energy sources are isolated prior to any construction, installation, system modification, repair, or maintenance activity. NIF Site Lockout/Tagout Requirements [17] describes the NIF standards for proper planning, authorization, shutting off, securing, and identifying energy sources to be isolated.

Key trees [18] are used in NIF to provide personnel entering or accessing the beampath a layer of protection from the optical hazards resulting from rod shots or main laser shots during operations or maintenance activities. Key trees disable the permissives required for charging the main laser Power Conditioning System (PCS) that supplies energy to the main amplifier flashlamps and the Power Conditioning Units (PCU) that supply energy to the Preamplifier Module flashlamps.

\section{Control of Beryllium and Radiological Hazards}

Appendix L of OSP 581.11 [19] describes the hazards and provides controls for managing radiation hazards and radioactive material at the NIF site and hazardous materials (particularly uranium and beryllium) used in NIF targets during NIF operations. The term radiation as used in this document refers solely to ionizing radiation (x-rays, gamma rays, neutrons, and charged particles). Since beryllium hazards are expected to be co-mingled with radiological material hazards, and controls for the two are similar, the two are treated together. Work permits and associated radiological/ beryllium work permits, when required, will specifically address the need to implement the associated requirements for the 


\section{National Ignition Campaign Program Completion Report}

intended work. Unless specifically approved otherwise by the NOM, all radiological and beryllium work in B581/582 will be conducted under these procedures. The Radiation Safety Officer is responsible for the radiological and beryllium control program.

\section{Argon, Liquid Nitrogen, and Oxygen Monitoring}

Elements of the argon system and the liquid nitrogen (LN) system are safety features. The argon system consists of a utility pad that stores liquid argon and conditions it for use within B581, and a distribution system that distributes it for use within the various NIF beampath enclosures. The critical safety-related functions of the argon system primarily center on confinement of argon, both on the pad and within B581.

The LN system is a storage and delivery system for cryogenic liquid nitrogen, which is

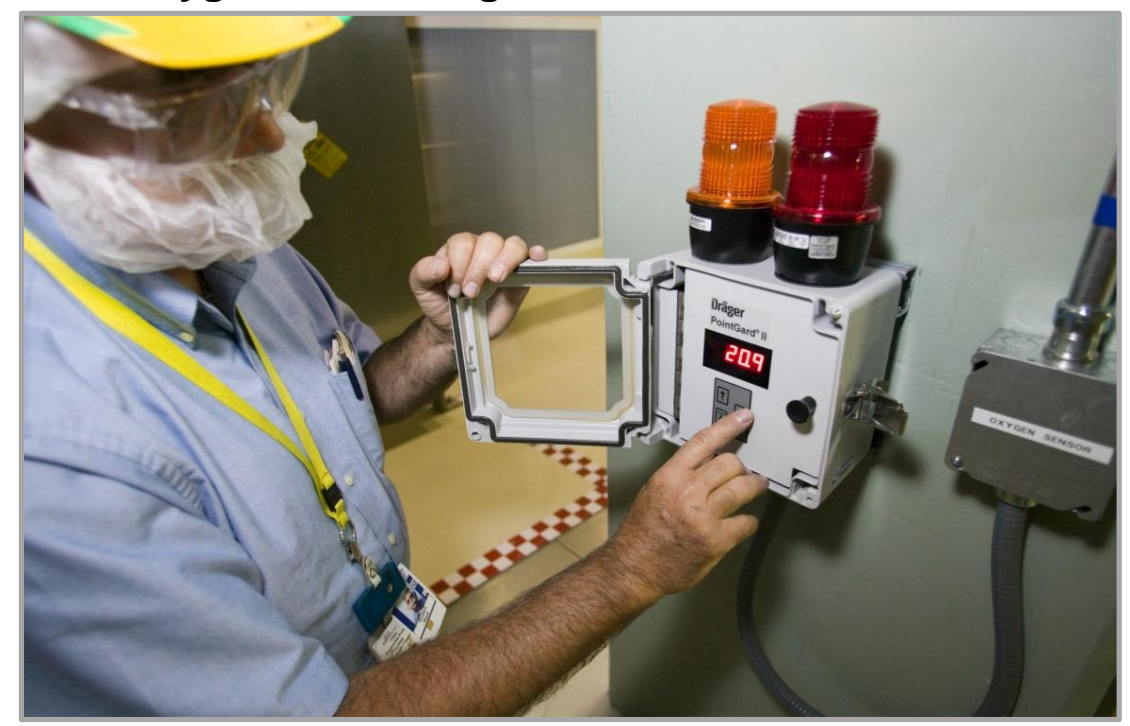

Figure 8-5. Technician tests one of the oxygen sensors in B581. supplied to the cryogenic pumps of the Target Area Vacuum (TAV) System. The LN system consists of a storage tank, piping, valves, and other components necessary to safely deliver liquid nitrogen to the cryogenic pumps of the TAV system. The LN System also includes vent piping to deliver the exhausted gaseous nitrogen from the cryogenic pumps to the stack. There are two critical safety functions for the LN system: confining nitrogen within the storage tank and distribution piping (i.e., supply and exhaust), and preventing damage to system components from over pressurization. Nitrogen confinement is necessary to protect workers from potential asphyxiation during oxygen-deficient conditions. The oxygen deficiency hazard is addressed by the monitoring and alarm system. This system monitors and alerts personnel when the oxygen level has dropped below 19.5 (see Figure 8-5).

\section{Fire Protection}

The NIF fire protection system is designed to contain and suppress a fire and to protect building occupants and equipment. The system provides fire detection and suppression, fire barriers to prevent the spread of fire and smoke, and alerts to personnel. The NIF is characterized by a level of fire protection sufficient to fulfill the requirements for the best-protected class of industrial risks, which qualifies it as an improved risk facility.

The fire barrier between the $\mathrm{OAB}$ and the LTAB is the one critical safety function of the fire protection system that is credited in the NIF Safety Basis [20]. This fire barrier, consisting of a four-hour-rated fire wall and two three-hour-rated fire rollup doors, essentially separates the OAB and LTAB into two distinct buildings for the purposes of fire hazard analyses. This barrier allows for a separate safety basis for each building area.

\section{Fracture Hazards}

The power conditioning system presents a shrapnel hazard during certain electrical failures. The module containing the power conditioning electrical components has been specially designed to vent overpressure and trap any shrapnel generated during such off-normal electrical events. Further, the Capacitor Bay walls have been reinforced to provide additional protection from any escaping shrapnel. The Beampath Vacuum Integrity System consists of various components related to vacuum-loaded fracture critical optics whose 


\section{National Ignition Campaign Program Completion Report}

failure could injure personnel working nearby in the facility. The system controls the hazard of large optics that also act as vacuum barriers. These optics may shatter due to flaws that grow from exposure to high-intensity light. An inspection system and pressure-mitigating features comprise the system.

\section{Laser Safety}

The hazard from NIF lasers is controlled by the laser safety system. In addition to the NIF main laser, there is a variety of other lower-power lasers used, such as for alignment and diagnostics. The laser safety system consists of a variety of barriers that protect personnel from exposure to any of these lasers. These barriers include specific beam blocks, shutters, laser curtains, and enclosures, as well as room walls and doors (e.g., laser bays).

Each of these systems has been evaluated in detail to understand the specific critical components required to ensure the functionality. Further, necessary maintenance or surveillance activities that support meeting the required function have also been specifically identified and monitored. This, combined with configuration management, ensures that safety function of these important systems is preserved.

\section{Safety Organization}

The goal of our safety program is to provide a safe and healthy work environment for our employees and visitors. A full-time staff of highly skilled environmental, safety, and health (ES\&H) professionals (health physicists, environmental analyst, safety engineers, industrial hygienist, health and safety technicians, and administrators), led by an ES\&H Manager is available at NIF. This team's role is to develop, monitor, and ensure safety and regulatory compliance of NIF operations. The importance of safety is embraced at all levels of NIF and ranks above all other aspects of our operations, including schedule and production.

Our ES\&H team provides guidance and assistance to ensure a safe working environment, including:

- Identifying and analyzing health, environmental, and safety exposures.

- Evaluating and providing guidance on ES\&H requirements, safety plans, environmental issues, permitting, and work authorization documents.

- Monitoring workplace compliance with ES\&H-related regulations.

- Training in occupational health, safety, and environmental areas.

- Responding to off-normal situations (chemical spills, fires, etc.).

- Investigating work-related injuries and illnesses.

\section{E. Integrated} Computer Control System Operations

One of the key operational accomplishments during NIC was demonstration and operation of NIF's Integrated Computer Control System (ICCS), the most complex, real-time control system ever designed for scientific research. Every NIF shot is a complex computerized coordination of laser equipment and the efforts of system operators according to laser settings calculated by LPOM. The automated ICCS system provides reliable

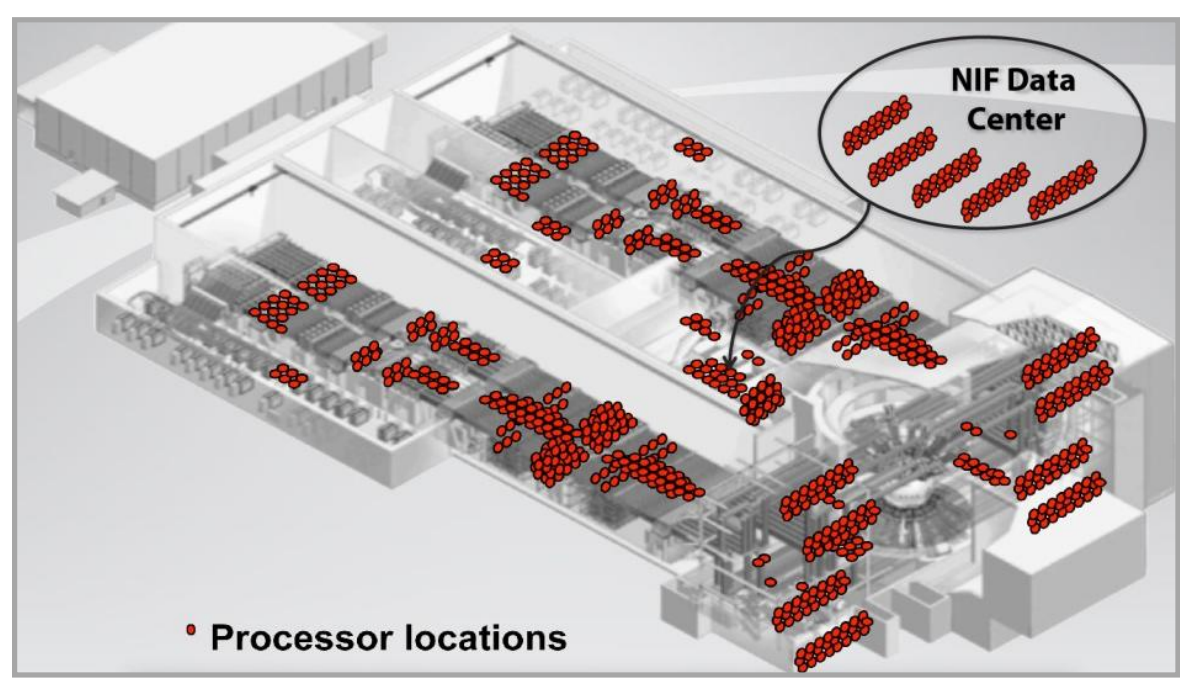

Figure 8-6. Layout of the NIF supervisory controls and front end processors. 


\section{National Ignition Campaign Program Completion Report}

monitoring and control of $\sim 60,000$ distributed control points composed of electronic, optical, and mechanical devices, such as motorized mirrors and lenses, adaptive optics, energy and power sensors, video cameras, and diagnostic instruments $[21,22]$ (see Figure 8-6). The precise orchestration of these parts results in a safe, accurate, and well-diagnosed laser shot.

\section{Design}

ICCS is a distributed, hierarchically organized control system that employs a scalable framework of reusable software to build uniform programs for beam control, injection laser, power conditioning, laser diagnostics, and target diagnostics [23]. Two principle layers comprise the control system - front end processors (FEP) attached to the laser hardware and supervisory controls that oversee the FEPs. ICCS architecture is partitioned into 24 bundles and distributed among over 1,800 FEPs and supervisory servers. Bundle control system partitions are replicated and commissioned by configuring the control database for each new bundle. Both layers are managed in the main NIF control room from an ensemble of operator consoles.

NIF's automated control subsystems are built from a common object-oriented software framework based on CORBA distribution that deploys the software across the computer network and achieves interoperation between different languages and target architectures. ICCS employs CORBA, Ada95, Java, and object-oriented techniques to enhance the openness of the architecture and portability of the software. Ada generally implements control system semantics. Java is used for the production of graphical user interfaces and the integration of commercial software, particularly the Oracle database system. CORBA provides transparent language binding and distribution middleware.

\section{Enhancements and Upgrades}

The ICCS framework supports large-scale control systems and accommodates the complexities of distribution through its flexible, modular design. The software architecture and CORBA middleware allow for easy reconfiguration of the computer control system by isolating independent control segments for upgrades or enhancements, without interrupting laser performance. Design modifications to implement bundle-based controls alleviated concerns that the computer and software architecture could scale successfully as NIF was

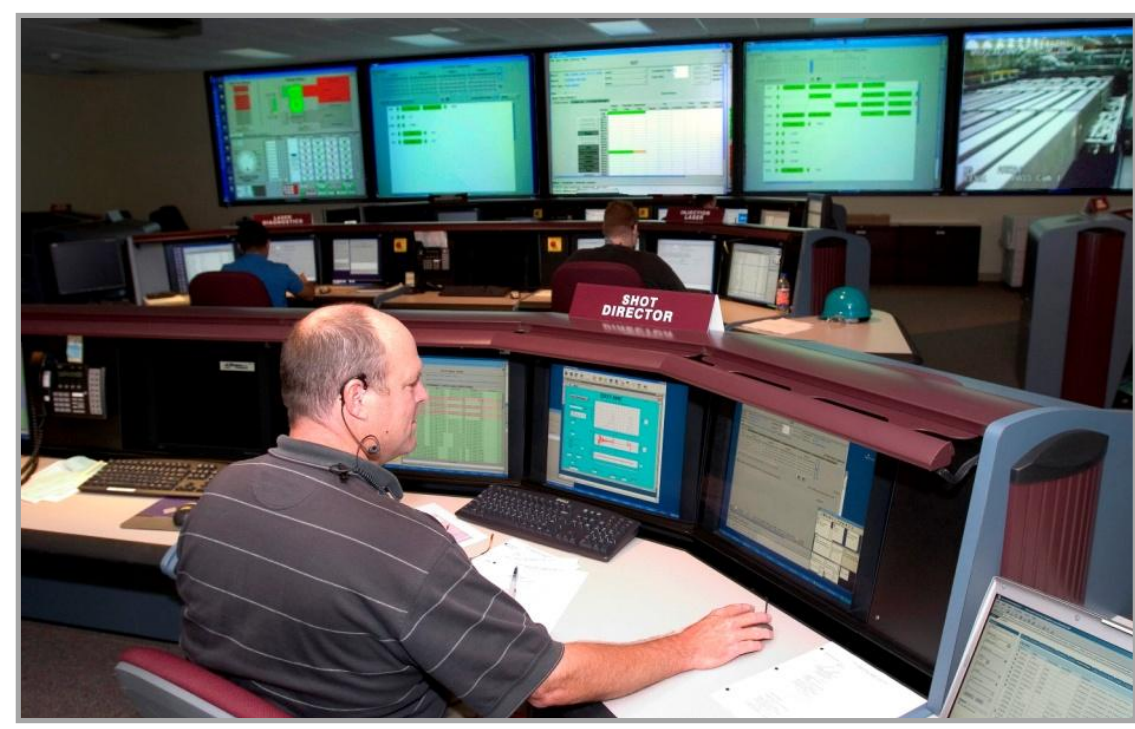

Figure 8-7. The NIF Shot Director oversees automated shot operations using the integrated computer control system.

built out. Model-driven shot automation software has successfully reduced efforts required of system operators, achieved the required shot rate, and enabled operational flexibility (see Figure 8-7).

The physical partitioning of NIF's independent bundles has been extended to the control system computer architecture. Control processes and computers were reorganized by bundle to achieve better parallelism, to assure predictable scaling performance, and to reduce the impact of localized failures. This is referred to as "bundle-based partitioning" and had no impact on framework or supervisory software due to the location-independent features of the CORBA distribution architecture. Bundle independence greatly simplifies the control system software because each bundle is operated asynchronously until the final countdown, at which point all systems are synchronized and fired by the master timing system. The 


\section{National Ignition Campaign Program Completion Report}

impact to the hardware architecture was to increase the number of FEPs by a factor of two and the number of servers to 24 sets (i.e., equal to the number of bundles). The cost of this modification was limited by replacing some planned servers and components in large FEPs with smaller, more inexpensive units. A large-scale, redundant gigabit Ethernet network upgrade was also performed to ensure reliability, to isolate the bundles from each other, and to deliver predictable scaling performance in the network backbone.

Over 300 sensor cameras in the beam control and laser diagnostics system were deployed as highresolution firewire cameras. A new PC-based FEP target architecture was added to leverage commercial code for firewire support. CORBA's language and processor transparency facilitated migration of the ICCS framework to Windows XP using an alternate Ada95 compiler technology (AdaCore GNAT Pro). The FEP can either capture shot data or deliver compressed streaming video to the operator consoles.

An automation framework was developed and deployed to automate bundle shots [24]. NIF's typical shot sequence includes shot lifecycle states (tasks) such as reading shot goals from LPOM, aligning laser beams, setting laser parameters, configuring diagnostics, verifying critical status readiness, and conducting a final four-minute countdown. The framework features a model-based workflow and provides scripted behaviors stored in the database that allow flexibility to modify automation instructions in the field without recompiling the software. The framework provides two major constructs to the application software: a workflow engine organizes collaboration among subsystem supervisors and coordinates transitions between shot lifecycle states and a master state machine that coordinates all bundle workflow engines. The automation framework operates 24 bundles in parallel by coordinating processes distributed over 750 processors.

\section{Quality Control}

Rigorous quality control processes established during the NIF Project continue to ensure the successful deployment of ICCS software releases [25]. A second test facility was constructed that shortened release delivery times by providing resources for integration in parallel with verification tests. The software inventory has grown to 3 million source lines of code, of which $20 \%$ is Ada and $80 \%$ Java. The code base is larger than initially estimated because new requirements were determined to include tool sets for supporting laser commissioning, diagnosing the distributed system in situ, and emulating devices for testing shot automation at scale in the test bed.

The ICCS team found the automated system to be more sensitive to software defects than manual controls. This was especially evident in distribution failure modes. Quality metrics were analyzed to help determine appropriate corrective actions. The data indicated additional developer testing and code inspections should be used to augment intensive integration and verification testing practices already in place. Results obtained by increasing the early-phase quality controls resulted in substantially more defects being found when they are less costly to repair. Consequently, nearly $95 \%$ of all defects are detected before ICCS software is used in laser operations. NIF experiments have validated NIF's control system design and automation requirements and have led to the identification and implementation of a number of enhancements and upgrades since the laser entered full operations.

\section{F. Ongoing Operational Improvement}

Now on the path to mature operations, NIF continues to evaluate and improve processes and capabilities, with the goal of maximizing availability, efficiency, and facility access while preserving safety. Initial facility controls were deliberately very conservative, but with a trained and stable workforce and roles, procedures, and protocols in place, controls are now bring revised to be commensurate with current hazards and reduce unnecessary overhead burden. Examples of recent efforts to revise facility controls include:

- Downgrading PPE requirements for general access, now that NIF is no longer a construction zone.

- Reevaluating the LOTO program; this saved over a thousand hours. 


\section{National Ignition Campaign Program Completion Report}

- Evaluating test and inspection periodicity, thereby greatly reducing the number of necessary tests.

- Evaluating the training and qualification card strategy, thus bringing training requirements in line with LLNL to reduce confusion and increase standardization.

- Reevaluating how many signatures are required and which roles must sign off for documents and permits.

- Reevaluating the configured items list for each CS to ensure it only included justified items.

- Evaluating and downgrading many radiological controls. The radiological operations workers' forum also contributed efficiency ideas, many of which were investigated and implemented.

- Evaluating pre-shot sweep protocol and moved from a manual to an automated sweep for certain shot types. These changes cut the sweep time for both laser bays and switchyards from 4 hours to 10 minutes, a major efficiency that allows for increased facility access between shots.

Examples of other efficiencies that have been successfully introduced to NIF operations include reducing the shot-to-shot cycle time by evaluating and optimizing each shot task and sequence and improving activities planning by developing an integrated shot and facility planning schedule that maximizes facility availability and minimizes diagnostic and optics reconfiguration. A new shot cycle metrics process for control room activities has been implemented to collect data from individual operators on delays to make additional improvements to shot cycle efficiency.

NIF has also been working to take a more strategic approach to maintenance, as an ever-increasing demand for shots continues to reduce facility maintenance windows. Working with technicians in the field to get feedback and recommendations on maintenance cycles has saved 400 hours a year by eliminating unnecessary maintenance. The effort to improve operational efficiency is an ongoing process that is maintained by management to continuously improve NIF's shot rate and increase the capabilities offered to the user community.

\section{References}

1. NIF Shot Operations Plan, NIF-5018506.

2. NIF Operations Management Plan, NIF-5020544.

3. NIF Maintenance Plan, NIF-5018526.

4. NIF Training Plan, NIF-5018705.

5. NIF RAM Process Documentation, NIF-0118248.

6. Final Site-wide Environmental Impact Statement for Continued Operation of Lawrence Livermore National Laboratory and Supplemental Stockpile Stewardship and Management Programmatic Environmental Impact Statement, DOE/EIS-0348, DOE/EIS-0236-S3, March 2005.

7. NIF Procedure 5.14, NIF Procedures and Plans Writers Guide, NIF-5018668.

8. NIF\&PS Configuration Management Plan, NIF-5018949.

9. What to do in an emergency, NIF-0064673.

10. Procedure 5.21, Off-Normal Event Response and Notification Process, NIF-5023077.

11. NIF Emergency Preparedness and Response Plan, NIF-5017354.

12. NIF\&PS Directorate Safety Manual, NIF-5032483.

13. Safety and Performance Review Board, Management Prestart Reviews, and Working Group Reviews, NIF-0115058.

14. Work Authorization Review Procedure, NIF-5018658.

15. NIF Work Permits, NIF-5018626.

16. NIF Clean Protocol, NIF-5022420.

17. NIF Procedure 5.15, NIF Site Lockout/Tagout Requirements, NIF-5018655.

18. Procedure 5.25, Use of Key Trees to Control Main Laser Optical Hazards on NIF, NIF- 5018474.

19. OSP 581.11, NIF Laser System Installation, Commissioning and Operation, NIF- 5017298.

20. NIF Safety Basis Document, NIF-5019666. 


\section{National Ignition Campaign Program Completion Report}

21. A. Heller, "Orchestrating the World's Most Powerful Laser," Science and Technology Review, Lawrence Livermore National Laboratory, UCRL-52000-05-7/8, July/August 2005.

22. P. Van Arsdall et al.," The National Ignition Facility: Status of the Integrated Computer Control System," ICALEPCS 2003, Gyeongjiu, Korea, October 2003.

23. R. Carey et al, "Status of the use of Large-Scale CORBA-Distributed Software Framework for NIF Controls," ICALEPCS 2005, Geneva, Switzerland, October 2005.

24. L. Lagin et al, "Shot Automation for the National Ignition Facility," ICALEPCS 2005, Geneva, Switzerland, October 2005.

25. D. Casavant et al.," Testing and Quality Assurance of the Control System during NIF Commissioning," ICALEPCS 2003, Gyeongjiu, Korea, October 2003. 


\section{National Ignition Campaign Program Completion Report}

\section{APPENDIX-ACRONYMS}

\begin{tabular}{|c|c|}
\hline Acronym & Definition \\
\hline AAMI & Automated ALARA MCNP Interface \\
\hline ACS & Access Control System \\
\hline AED & Automated External Defibrillator \\
\hline AFM & Atomic force microscopy \\
\hline ALARA & As low as reasonably achievable \\
\hline AMP & Advanced Mitigation Process \\
\hline ARIANE & Active Readout in a Neutron Environment \\
\hline ARC & Advanced Radiography Capability \\
\hline AWE & Atomic Weapons Establishment \\
\hline BLIP & Beamline and Laser Integrated Performance \\
\hline BUTrY & Beryllium/uranium/tritium/yield \\
\hline $\mathrm{CCD}$ & Charge-coupled device \\
\hline CCI & Cleveland Crystals Incorporated \\
\hline CEA & Commissariat a l'Energie Atomique \\
\hline CFTA & Capsule fill tube assembly \\
\hline $\mathrm{CM}$ & Configuration management \\
\hline CMT & Campaign Management Tool \\
\hline $\mathrm{CR}$ & Compton radiography \\
\hline $\mathrm{CR}$ & Contact radiography \\
\hline $\mathrm{CS}$ & Configured system \\
\hline CTS & Cryogenic Target System \\
\hline CTXD & $\begin{array}{l}\text { CryoTARPOS Load, Layering, and } \\
\text { Characterization X-ray Detector }\end{array}$ \\
\hline CVD & Chemical vapor deposition \\
\hline DCS & Diagnostic Control System \\
\hline DDS & Disposable Debris Shield \\
\hline DIM & Diagnostic Instrument Manipulator \\
\hline DIXI & Dilation X-ray Imager \\
\hline DISC & DIM Insertable Streak Camera \\
\hline DKDP & Deuterated potassium dihydrogen phosphate \\
\hline DOE & Department of Energy \\
\hline DSF & Downscattered fraction \\
\hline DSR & Downscattered ratio \\
\hline DT & Deuterium, tritium \\
\hline DU & Depleted uranium \\
\hline EDS & Energy dispersive x-ray spectroscopy \\
\hline EHXI & Equatorial Hard X-ray Imager \\
\hline EMP & Electromagnetic pulse \\
\hline EOS & Equation of state \\
\hline EP & Execution Plan \\
\hline ES\&H & Environment, safety and health \\
\hline FABS & Full-Aperture Backscatter System \\
\hline FEP & Front end processor \\
\hline FFLEX & Filter Flourescer \\
\hline FIB & Focused ion beam \\
\hline FLIP & Facility Laser Interactive Planning \\
\hline
\end{tabular}

\begin{tabular}{|c|c|}
\hline Acronym & Definition \\
\hline FMEA & Failure modes and effects analysis \\
\hline FOA & Final Optics Assembly \\
\hline FODI & Final Optics Damage Inspection \\
\hline FR & Functional Requirement \\
\hline FSE & Functional Safety Expert \\
\hline GA & General Atomics \\
\hline GAM & Gamma area monitoring \\
\hline GDS & Grating debris shield \\
\hline GRH & Gamma Reaction History \\
\hline GUI & Graphical user interface \\
\hline GXD & Gated X-ray Detector \\
\hline HED & High-energy-density \\
\hline HEDSS & High Energy Density Stewardship Science \\
\hline hGXI & Hardened, Gated X-ray Imager \\
\hline HMMA & Hazardous Materials Management Area \\
\hline ICCS & Integrated Computer Control System \\
\hline ICF & Inertial confinement fusion \\
\hline ILS & Injector Laser System \\
\hline ITF & Ignition Threshold Factor \\
\hline I-TIC & Ignition Target Inserter and Cryostat \\
\hline ITPS & Ignition Target Proofing Station \\
\hline IWS & Integrated Work Order \\
\hline JHA & Job Hazard Analysis \\
\hline KDP & Potassium dihydrogen phosphate \\
\hline LANL & Los Alamos National Laboratory \\
\hline LDRD & $\begin{array}{l}\text { Laboratory Directed Research and } \\
\text { Development }\end{array}$ \\
\hline LEH & Laser entrance hole \\
\hline LLCS & $\begin{array}{l}\text { Load, Layering, and Characterization } \\
\text { System }\end{array}$ \\
\hline LLE & Laboratory for Laser Energetics \\
\hline LLNL & Lawrence Livermore National Laboratory \\
\hline LMJ & Laser MegaJoule \\
\hline $\mathrm{LN}$ & Liquid nitrogen \\
\hline LoCoS & Location Component and State \\
\hline LPI & Laser-plasma interaction \\
\hline LPOM & Laser Performance Operations Model \\
\hline MCNP & Monte-Carlo N-Particle \\
\hline $\mathrm{MCP}$ & Micro-channel plate \\
\hline MPR & Management Prestart Review \\
\hline MRF & Magneto-rheological finishing \\
\hline MRS & Magnetic Recoil Spectrometer \\
\hline MTE & Major technical effort \\
\hline MVSS & Multivariable sensitivity study \\
\hline NAD & Neutron Activation Detector \\
\hline NBI & Near-Backscatter Imager \\
\hline
\end{tabular}




\section{National Ignition Campaign Program Completion Report}

\begin{tabular}{|l|l|}
\hline Acronym & Definition \\
\hline NEET & NIF Exposure Estimation Tool \\
\hline NEPA & National Environmental Policy Act \\
\hline NIC & National Ignition Campaign \\
\hline NIF & National Ignition Facility \\
\hline NIS & Neutron Imaging System \\
\hline nITOF & Neutron imager time-of-flight \\
\hline NNSA & National Nuclear Security Administration \\
\hline NOL & NIF Optics Loop \\
\hline NOM & NIF Operations Manager \\
\hline NPS & NIF Planning System \\
\hline nTOF & Neutron Time-Of-Flight \\
\hline OMF & Optics Mitigation Facility \\
\hline OPAS & Opposed Port Alignment System \\
\hline OSP & Output Sensor Package \\
\hline PC & Primary Criteria \\
\hline PCC & Program Completion Criteria \\
\hline PCS & Power Conditioning System \\
\hline PCU & Power Conditioning Units \\
\hline PD & Polar Drive \\
\hline PEPC & Plasma electrode Pockels cell \\
\hline PEPS & $\begin{array}{l}\text { Personnel and Environmental Protection } \\
\text { Systems }\end{array}$ \\
\hline PI & Principal Investigator \\
\hline PL & Photo luminescence \\
\hline PM & Preventive Maintenance \\
\hline PPE & Personnel protective equipment \\
\hline PR & Polarization rotators \\
\hline PSDI & Phase shifting diffractive interferometry \\
\hline pTOF & Particle Time-of-Flight \\
\hline PZT & Piezoelectric transducer \\
\hline RA & Readiness Assessment \\
\hline RAGS & $\begin{array}{l}\text { Radiochemistry Apparatus for Gas } \\
\text { Sampling }\end{array}$ \\
\hline RAM & Reliability, availability, and maintainable \\
\hline RCM & Reliability-centered maintenance \\
\hline RCT & Radiological control technician \\
\hline RS & Responsible Scientist \\
\hline RT & Safety Bastrisis Document \\
\hline SAVI & \\
\hline SBD & SD \\
\hline
\end{tabular}

\begin{tabular}{|l|l|}
\hline Acronym & Definition \\
\hline SEM & Scanning electron microscopy \\
\hline SHG & Second-harmonic-generator \\
\hline SIS & Safety Interlock System \\
\hline SLMP & System-level maintenance plan \\
\hline SMaRT & $\begin{array}{l}\text { Systems Maintenance and Reliability } \\
\text { Tracking }\end{array}$ \\
\hline SNL & Sandia National Laboratories \\
\hline SOP & Streaked Optical Pyrometer \\
\hline SPBT & South Pole Bang Time (diagnostic) \\
\hline SPIDER & $\begin{array}{l}\text { Streaked Polar Instrumentation for } \\
\text { Diagnosing Energetic Radiation }\end{array}$ \\
\hline SRC & Solid Radiochemical Collector \\
\hline SSP & Stockpile Stewardship Program \\
\hline SXI & Static X-ray Imager \\
\hline TaLIS & Target and laser interaction \\
\hline TARPOS & Target Positioner \\
\hline TAV & Target Area Vacuum \\
\hline TCC & Target Chamber Center \\
\hline TEM & Transmission electron microscope \\
\hline TGM & Target Gas Manifold \\
\hline THD & Tritium, hydrogen, deuterium \\
\hline THG & Third-harmonic generator \\
\hline TMP & Thermal mechanical package \\
\hline TMS & Tritium Monitoring System \\
\hline TPS & Tritium Processing System \\
\hline TRC & Technical Review Committee \\
\hline VBL & Virtual beamline \\
\hline VISAR & $\begin{array}{l}\text { Velocity Interferometer System for Any } \\
\text { Reflector }\end{array}$ \\
\hline WAP & Work Authorization Point \\
\hline WBS & Work Breakdown Structure \\
\hline WFL & Wedge focus lens \\
\hline WPRI & Work Permit Responsible Individual \\
\hline WRF & Wedge Range Filter \\
\hline XRF & X-ray fluorescence \\
\hline YOC & Yield over clean \\
\hline
\end{tabular}


National Ignition Campaign Program Completion Report 